\title{
Algorithmen zur Kopplung und Interpolation in der Aeroelastik
}

\author{
Dissertation \\ zur Erlangung des Doktorgrades \\ der Mathematisch-Naturwissenschaftlichen Fakultäten \\ der Georg-August-Universität zu Göttingen
}

vorgelegt von

Regine Ahrem

aus Wuppertal

Göttingen 2005 
D7

Referent: PD Dr. Holger Wendland

Korreferent: Prof. Dr. Robert Schaback

Tag der mündlichen Prüfung: 
Meiner Großmutter

Brunhilde Kehrenberg in Liebe und Dankbarkeit gewidmet. 


\section{Vorwort}

Das Entstehen dieser Dissertation ist wesentlich begründet im HGF-Projekt AMANDA (A Multidisciplinary High Performance Numerical Development System for Aircraft) [1], das von mehreren Instituten des Deutschen Zentrums für Luft- und Raumfahrt (DLR) zusammen mit dem Institut für Algorithmen und Wissenschaftliches Rechnen (SCAI) der Fraunhofer-Gesellschaft durchgeführt wurde. Ein Ziel von AMANDA war die Entwicklung einer Softwareumgebung, welche die Simulation eines freifliegenden, elastischen Großraumflugzeugs im stationären Betriebszustand ermöglicht. Die Aufgabe, dieses multidisziplinäre Problem zu bearbeiten, erforderte das Zusammenspiel mehrerer Simulationscodes verschiedener Disziplinen. So arbeiteten an diesem Projekt Mitarbeiter von Instituten unterschiedlicher Fakultäten, die ihre jeweiligen Simulationstools und ihr Spezialwissen eingebracht haben.

Um die Vorgänge bei einem frei fliegenden Flugzeug simulieren zu können, müssen die entstehenden Wechselwirkungen zwischen den auf das Flugzeug wirkenden, verschiedene monodisziplinäre Felder betreffenden, physikalischen Gesetzen berücksichtigt werden. Da die numerischen Verfahren, die zur Simulation des monodisziplinären Verhaltens angewendet werden, auf unterschiedlichen Diskretisierungsverfahren partieller Differentialgleichungen beruhen, ist für den Erfolg eines solchen Projektes ein sogenanntes Kopplungstool von entscheidender Bedeutung. Dieses Programm muss den Austausch von Daten in entsprechender zeitlicher Abfolge und deren Anpassung an das jeweils andere Simulationsverfahren realisieren. Am Institut für Aeroelastik des DLR ist von Beckert ein solches Kopplungstool mit dem Namen CAESAR speziell für die Strömung-Struktur-Kopplung bei Flugzeugen ([2], [3]) entwickelt worden. Das Werkzeug beinhaltet Algorithmen zur Kopplung in Raum und Zeit. Diese mathematischen Verfahren stellen die Kernelemente bei der numerischen Lösung gekoppelter Probleme dar. Für die räumliche Kopplung existieren zwei Arten von speziell angepassten Interpolationsalgorithmen. Ein Algorithmus arbeitet mit finiten Interpolationselementen und ein zweiter führt die Interpolationen auf Grundlage von radialen Basisfunktionen [4] aus. Darüber hinaus enthält das Tool effiziente Kopplungsalgorithmen für statische und dynamische Probleme.

Am Fraunhofer-Institut SCAI wurde die Kopplungsbibliothek MpCCI (Mesh-based parallel Code Coupling Interface) [5] entwickelt, welche allgemein zur Lösung multidisziplinärer Probleme verwendet wird. Über MpCCI können, wie mit CAESAR, vorhandene monodisziplinäre Codes gekoppelt werden, wobei integrierte Standard-Interpolationsalgorithmen für die räumliche Kopplung zur Verfügung stehen. Die Kopplungsbibliothek MpCCI ist MPI (Message Passing Interface) basiert, so dass ein effizienter, schneller Da- 
tenaustausch möglich ist. Die Kopplungsalgorithmen müssen hier von außen über eine Abfolge von MpCCI-Aufrufen aufgesetzt werden.

Im Projekt zeigte sich, dass zum einen die speziell angepassten Algorithmen aus CAESAR benötigt werden, zum anderen für ein dynamisches Problem ein schneller Datenaustausch jedoch unabdingbar ist. Da der Datenaustausch in CAESAR dateibasiert ist, ging die Überlegung dahin, MpCCI als softwaretechnische Grundlage mit den Interpolationsverfahren aus CAESAR zu nutzen. Darüber hinaus wurde geplant, die bestehenden Algorithmen zu parallelisieren und verfeinerte Methoden auf Grundlage radialer Basisfunktionen zu entwickeln und zu untersuchen. Die Algorithmen sollten verbesserte Resultate liefern und die Rechenzeiten sowie den Speicherplatzbedarf bei unveränderter Qualität senken. Dieses sind die Aufgaben des zu diesem Zweck vom Institut für Aeroelastik des DLR und des Fraunhofer-Instituts SCAI definierten Kooperationsprojekts mit dem Namen AKITA (Algorithmen zur Kopplung und InTerpolation in der Aeroelastik) und stellen im wesentlichen die Schwerpunkte der vorliegenden Dissertation dar. Die Weiterentwicklung der Interpolationsalgorithmen wurde in Zusammenarbeit mit Priv. Doz. Dr. Holger Wendland vom Institut für Numerische und Angewandte Mathematik der Georg-August-Universität Göttingen und Dr. Armin Beckert von der European Aeronautic Defence and Space Company (EADS) durchgeführt. Das Interpolationstool, das Beckert am Institut für Aeroelastik des Deutschen Zentrums für Luft- und Raumfahrt implementiert hat, stellt den Ausgangspunkt dieser Dissertation dar.

Das Ziel des Forschungsvorhabens ist die Entwicklung von effizienten und allgemein einsetzbaren Interpolationen mit radialen Basisfunktionen zur räumlichen Kopplung von Strömungs- und Strukturmodellen im Bereich der Aeroelastik, sowie deren softwaretechnische Implementierung als separates Software-Modul (kompatibel zur MpCCI-Programmier-Schnittstelle) und Erprobung in der Praxis.

Bei Interpolationen, die nicht auf Elementstrukturen zurückgreifen können oder die spezielle Zusatzbedingungen erfordern, erweisen sich Verfahren mit radialen Basisfunktionen als sehr nützlich und teilweise unersetzlich. Der große Vorteil der Interpolationen mit radialen Basisfunktionen ist, dass sie keinerlei Struktur erfordern und trotzdem die gewünschte Glattheit sowie Zusatzbedingungen erfüllen können. Mit radialen Basisfunktionen werden Rekonstruktionsfunktionen erstellt, so dass für jeden Punkt im Raum der Interpolationswert über diese Funktion abgegriffen werden kann. Damit ist der Raum der Auswertungspunkte unbegrenzt. Mit radialen Basisfunktionen sind Interpolationen im n-dimensionalen Raum möglich, was sie auch für Interpolationen von größeren Mengen von Parameterwerten anwendbar macht.

Bereits im Projekt AMANDA sind verschiedene Codes über MpCCI und die Interpolationsalgorithmen aus CAESAR gekoppelt worden, wobei die Interpolationsalgorithmen mit Hilfe der im Projekt AKITA entwickelten, parallelen Schnittstellen an MpCCI angeschlossen waren. Der am Institut für Aeroelastik entwickelte Multibody Simulationscode SIMPACK hat mit dem 
Computational Fluid Dynamics Code FLOWer des DLR-Instituts für Aerodynamik und Strömungstechnik gekoppelte Berechnungen durchgeführt. SIMPACK berechnete die elastischen Verformungen auf Basis von vorherigen Modalanalysen aus dem Finite Elemente Strukturlöser NASTRAN.

Die Daten aus aeroelastischen Anwendungen resultieren aus Berechnungen von Beckert und Neumann am Institut für Aeroelastik mit dem schon genannten Finite Elemente Strukturlöser NASTRAN zur detaillierten Spannungs- und Verformungsvorhersage und dem zur Simulation der Strömungsphänomene eingesetzten Code TAU.

Die in dieser Arbeit präsentierten gekoppelten Simulationen sind von Beckert und Mattioni bei EADS berechnet worden. Der hier eingesetzte Strukturlöser ist der EADS Inhouse Code LAGRANGE. LAGRANGE ist speziell für die Simulation von Flugzeugen entwickelt worden. Die verwendeten Strömungscodes sind AIRPLANE, der ebenfalls bei EADS entwickelt worden und speziell auf die Flugzeugberechnung ausgerichtet ist, und der vorher genannte Strömungscode TAU. 
VIII Vorwort 


\section{Inhaltsverzeichnis}

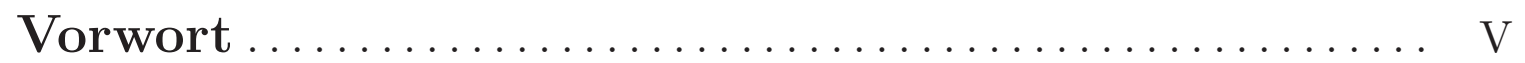

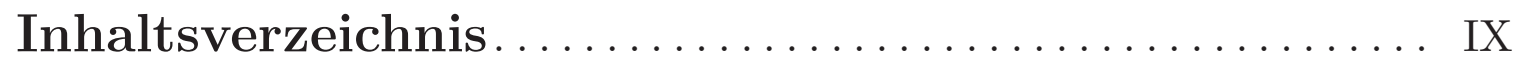

I. Einleitung $\ldots \ldots \ldots \ldots \ldots \ldots \ldots \ldots \ldots \ldots \ldots \ldots \ldots \ldots \ldots \ldots \ldots \ldots$

II. Radiale Basis Funktionen ..................... 12

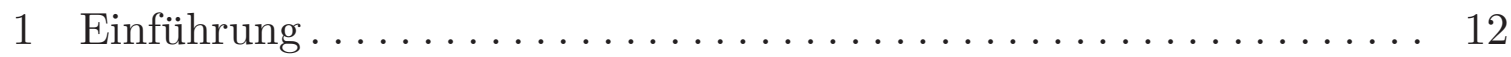

2 Zusatzbedingungen für die Kopplung in der Aeroelastik .......... 21

2.1 Erhaltung der Arbeit ......................... 22

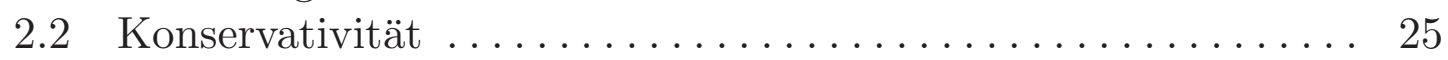

2.3 Erhaltung der Arbeit und Konservativität ............ 27

2.4 Rotationen ............................... 29

3 Das „Partition of Unity" Verfahren ...................... 32

3.1 Allgemeine Überlegungen . . . . . . . . . . . . . . . . . . 32

3.2 Algorithmen zur Aufteilung des Gebietes in Zellen. . . . . . . . . 35

3.3 Komplexität des Algorithmus .................... 38

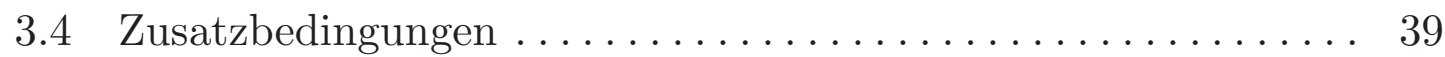

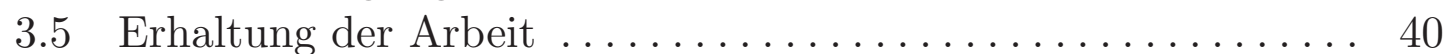

3.6 Konservativität .......................... 42

3.7 Erhaltung der Arbeit und Konservativität ............. 44

3.8 Rotationen ................................ 45

III. Softwaretechnische Realisierung ............. 48

1 Die Kopplungsbibliothek MpCCI....................... 48

1.1 Konzept................................. 48

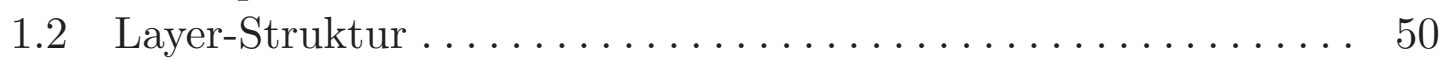

1.3 Kopplungsphasen........................... 52

1.4 Zusätzliche Tools . . . . . . . . . . . . . . . . . . . . . 59

1.5 Standardinterpolationen ........................ 59

1.6 Funktionen für User-Subroutines . . . . . . . . . . . . . . . . 64

2 Die Interpolationsbibliothek AKITA . . . . . . . . . . . . . . 67

2.1 Problembeschreibung .......................... 67

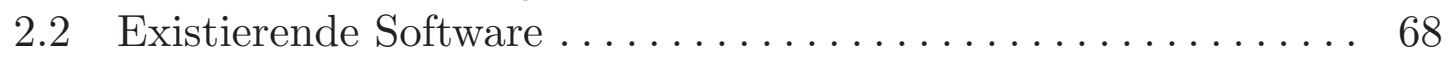

2.3 Datenumwandlung und Parallelisierung . ............. 69

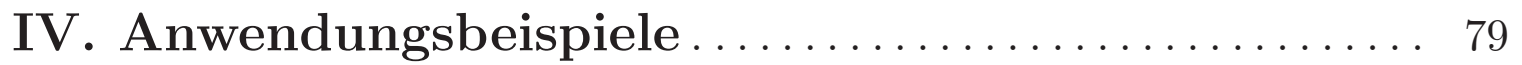

1 Anwendungen des parallelen Interfaces................ 80

1.1 Strömung-Struktur-Kopplung am AGARD Flügel ......... 80

2 Anwendungen zu Arbeitserhaltung und Konservativität ......... 85 
2.1 Strömung-Struktur-Kopplung am AGARD Flügel . . . . . . . 85

3 Anwendungen unter Einbeziehung der Rotationen . . . . . . . . . . . . 87

3.1 Strömung-Struktur-Kopplung am AMP Flügel . . . . . . . . . . . . 87

4 Anwendungen des "Partition of Unity" Verfahrens . . . . . . . . . . . 94

4.1 Das A340-300 Halbmodell - Ein analytischer Testfall . . . . . . . . 94

4.2 Das A340-300 Halbmodell - Ein realistischer Testfall . . . . . . . . 98

4.3 Datenaustausch in der Rohrleitung . . . . . . . . . . . . . . . 100

4.4 Datenaustausch in der Sprühlackierung . . . . . . . . . . . . . . 104

4.5 Datenaustausch in der Umformtechnik . . . . . . . . . . . . . . . . 109

5 Anwendungen des PoU Verfahrens mit Zusatzbedingungen . . . . . . . 112

5.1 Strömung-Struktur-Kopplung am ALENIA Halbmodell . . . . . 112

6 Anwendungen des PoU Verfahrens mit Rotationen . . . . . . . . . . . 121

6.1 Strömung-Struktur-Kopplung am ALENIA Halbmodell . . . . . 121

7 Gekoppelte Simulation in der Aeroelastik . . . . . . . . . . . . . . 127

7.1 Das Strömung-Struktur-Kopplungsverfahren . . . . . . . . . . . 127

Strukturgleichungen . . . . . . . . . . . . . . . . . . . . . . . . . . . 128

Strömungsgleichungen . . . . . . . . . . . . . . . . . . 129

Struktur- und Strömungsmodell . . . . . . . . . . . . . . . 129

Lösungsalgorithmus . . . . . . . . . . . . . . . . . . . 133

7.2 Simulationsgrundlagen . . . . . . . . . . . . . . . . . . . 134

Testfälle. . . . . . . . . . . . . . . . . . . . . . . 135

7.3 Simulationsergebnisse . . . . . . . . . . . . . . . . . 141

Rechenzeiten......................... 144

V. Zusammenfassung und Ausblick ............... 154

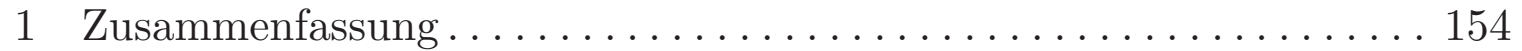

2 Diskussion ..................................... 155

3 Ausblick ............................. 157

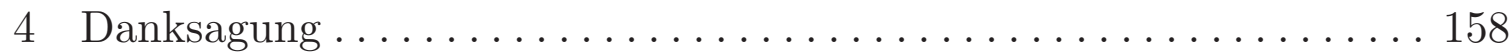

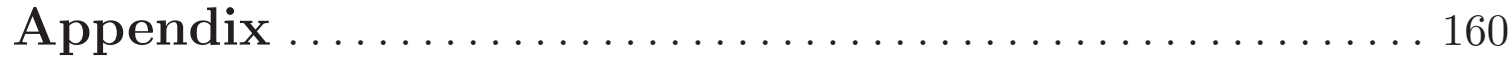

A Tabellen zu den Abbildungen der Anwendungen .............. 160

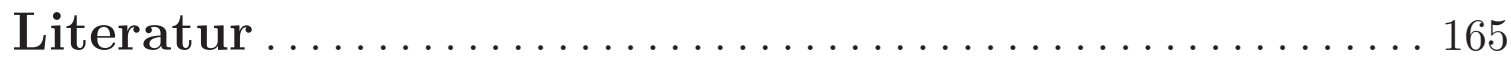




\section{Einleitung}

Problemstellung. Eine Vielzahl von Entwicklungen zum Beispiel in den Bereichen medizinische Forschung, Bau von Brücken und Ölplattformen oder Flugzeug- und Automobilbau erfordern gekoppelte multidisziplinäre Simulationsverfahren. Beispielsweise sind Herzklappen, Tragflügel, Triebwerke oder auch Drehmomentwandler in ihrem Einsatz starken Belastungen durch die andauernden Wechselwirkungen zwischen Strömung und Struktur ausgesetzt, denen ihre Strukturkomponenten standhalten müssen.

Die Simulation der Wechselwirkung zwischen einer Strömung und einer bewegten, deformierbaren Struktur ist eine häufige und wichtige Aufgabe in vielen ingenieurtechnischen, wissenschaftlichen Problemen. Die allgemeine Bezeichnung solcher Probleme ist Strömung-Struktur-Wechselwirkung (SSWW), ein Begriff der Hydroelastik und Aeroelastik einschließt. Im Zentrum dieser Arbeit liegt die SSWW-Simulation von dreidimensionalen Tragflügelstrukturen.

Solche „Multi-Physics Systeme“ gewinnen zunehmend an Bedeutung, da es die Simulationsmöglichkeiten infolge der steigenden Rechnerkapazitäten und neuen numerischen Methoden seit einigen Jahren erlauben, auch solche Probleme anzugehen. Zudem stellt die Fähigkeit zur computergestützten Simulation als einem zentralen Teil des CAE (Computer-Aided-Engineering) bei der Entwicklung aller Arten von Bauteilen einen entscheidenden Wettbewerbsvorteil infolge einer deutlichen Senkung der Entwicklungsdauer und des Aufwandes bei gleichzeitig verbesserter Produktqualität dar.

Viele komplexe physikalische Phänomene können als gekoppelte Probleme betrachtet werden. Diese sind Kombinationen von mindestens zwei Problemen, die durch Systeme von partiellen Differentialgleichungen beschrieben werden. Die Berechnung jedes einzelnen Aspektes des Problems wird gewöhnlich auf unterschiedlichen, aber miteinander verbundenen Bereichen durchgeführt. Für die monodisziplinären physikalischen Probleme existieren effiziente parallelisierte Simulationscodes auf einem hohen technologischen Standard.

Der Ansatz, der in dieser Arbeit zum Tragen kommt, ist die lose Kopplung der Teilberechnungen. Bei der losen Kopplung werden die unterschiedlichen physikalischen Aspekte getrennt berechnet und die jeweils benötigten Werte zwischen den Teilbereichen ausgetauscht. Diese neuen „Randbedingungen" gehen dann in den nächsten Berechnungsschritt des jeweiligen Simulationscodes ein. Die genaue Abfolge des Austauschs der Werte und der Berechnungsschritte wird durch einen Kopplungsalgorithmus geregelt. Bei einem aeroelastischen Problem werden vom Strömungscode (Computational Fluid Dynamics Code) Kräfte zum Strukturcode (Computational Structure Mechanics) gegeben, und der Strukturcode wiederum berechnet aus diesen 
Kräften eine Verformung, die an den Strömungscode zurückgegeben wird. Der Strömungscode ermittelt unter Berücksichtigung der Verformung die Strömung und die neue Druck- bzw. Kräfteverteilung an der Oberfläche der Struktur. Für die Kopplung sind immer nur die Teile der Gitter interessant, auf denen Werte ausgetauscht werden müssen, da diese Teile durch einen anderen physikalischen Aspekt beeinflusst werden als ihn die gerade betrachtete Simulation bearbeitet. So sind im Folgenden mit den verschiedenen Gittern immer die Teile der Gitter gemeint, auf denen Kopplungswerte ausgetauscht werden.

Für eine vollständige Analyse des aeroelastischen Deformationsverhaltens eines SSWW-Problems auf dem Rechner sind spezielle Simulationscodes erforderlich. Diese numerischen Simulationsprogramme arbeiten für die Teilberechungen auf unterschiedlichen Diskretisierungen. Beim Austausch der Kopplungsgrößen zwischen den Gittern der beteiligten Simulationscodes müssen diese zugrundeliegenden Diskretisierungen der Codes berücksichtigt werden. Die Anwendbarkeit einer Kopplung wird zu einem großen Teil von den verfügbaren Interpolationen bestimmt. In besonderem Maße werden gerade in der Aeroelastik bei den speziellen Kopplungen komplexe, problemspezifische Interpolationen benötigt. In dieser Dissertation werden, aufbauend auf bestehenden Interpolationen, speziell angepasste Verfahren unter Verwendung radialer Basisfunktionen (siehe [6] und [4]) entwickelt, implementiert und anhand von konkreten Testfällen validiert.

Vorgehensweise. Ausgangspunkt der vorliegenden Dissertation ist zum einen das von Beckert am Institut für Aeroelastik des DLR entwickelte und implementierte Kopplungstool CAESAR ([2], [7]) und zum anderen die Kopplungsbibliothek MpCCI [8], die am Fraunhofer-Institut SCAI entwickelt wurde.

MpCCI bietet bislang lineare, bilineare und quadratische Standardinterpolationen an, jeweils mit und ohne Bedingung zur Konservativität. Konservativität bedeutet in diesem Zusammenhang, dass die Summe der interpolierten Größe erhalten bleibt. Diese Standardinterpolationen reichen für aeroelastische Problemstellungen allerdings nicht aus. Die Interpolationsalgorithmen in CAESAR sind hingegen speziell für die Anforderungen in der Aeroelastik implementiert worden. Eines der Verfahren setzt auf der Methode der finiten Interpolationselemente auf, die übrigen verwenden verschiedene radiale Basisfunktionen als Grundlage. Gekoppelte Simulationen unter Verwendung von CAESAR mit realen Testkonfigurationen zeigten eine gute Übereinstimmung mit experimentell gewonnenen Daten.

Die Interpolationsalgorithmen aus CAESAR werden in dieser Arbeit in MpCCI integriert. MpCCI realisiert einen schnellen und effektiven Datenaustausch insbesondere zwischen parallelen Simulationscodes. MpCCI ist eine mit Methoden des modernen Software-Engineering entwickelte Kommunikationsbibliothek. Kombiniert mit den speziellen Interpolationen von CAESAR wird MpCCI angewendet zur Kopplung von Strömungscodes wie FLOWer und TAU mit Strukturcodes, wie die auf der Finite Elemente Methode ba- 
sierenden Codes NASTRAN und Lagrange oder die Multibody-Simulation SIMPACK.

Der erste Schritt im vorliegenden Projekt zur Anbindung der Interpolationsalgorithmen an MpCCI bestand darin, die Routinen aus CAESAR in ein eigenständiges Modul KopMat zur Erstellung der räumlichen Kopplungsmatrix mit entsprechender Schnittstelle herauszulösen.

Danach wurde das Kopplungstool KopMat an die Kopplungsbibliothek MpCCI über eine neue Schnittstelle angeschlossen. Da MpCCI bislang noch nicht über eine Möglichkeit zur Nutzung einer benutzerdefinierten Interpolation verfügte, wurden zunächst Funktionen implementiert, die den Eingriff an geeigneter Stelle ermöglichen. Mit diesen Funktionen können zum einen die Informationen über die Gitter erfragt werden und zum anderen die Interpolationsmatrizen für die jeweiligen Partitionspaare auf Sender- und Empfängerseite an MpCCI spezifiziert werden.

Im ersten Ansatz wurde KopMat aus einer entsprechend definierten Funktion per Systemaufruf gestartet und erhielt über Input-Dateien die Gitterinformationen von MpCCI; die Kopplungsmatrix ging dann auf die gleiche Weise an die Funktion zurück, welche sie für die jeweiligen Partitionspaare aufteilte und über die oben beschriebene Schnittstellenfunktion an MpCCI definierte. Im darauf folgenden Schritt wurde die dateibasierte Lösung ausgetauscht. KopMat wurde in eine Bibliothek umgewandelt, aus der die benötigten Funktionen aufgerufen wurden.

Anschließend wurden die beiden unterschiedlichen Arten von Interpolationsverfahren in KopMat auf zwei getrennte Software-Bibliotheken aufgeteilt. Der Teil des räumlichen Kopplungstools, der die Werte auf Grundlage radialer Basisfunktionen interpoliert, wurde in $\mathrm{C}++$ umgeschrieben und mit ABSCL (Armin Beckert Scientific Coupling Library) bezeichnet. Dieses Interpolationstool wurde zusätzlich zu KopMat wiederum über die Schnittstellenfunktionen als Bibliothek an MpCCI angeschlossen. Es ersetzt komplett die Funktionalität von KopMat bezüglich der genannten Interpolationen auf Grundlage radialer Basisfunktionen. Die Interpolationen über finite Interpolationselemente [3] wurden aus der Umgebung der radialen Basisfunktionen genommen und zu einer eigenen Bibliothek mit Schnittstelle zu MpCCI zusammengebunden.

Die parallele Situation der Codes und die daraus folgende Partitionierung der Gitter regelt die Schnittstelle zu ABSCL bzw. KopMat. Mehrfach vorkommende Punkte, die durch die Partitionierung auftreten, werden auf der Stützstellen-Seite herausgefiltert. Die Aufteilung der Kopplungsmatrix sowie der transponierten Kopplungsmatrix auf die Matrizen der Paare von Senderund Empfängerpartitionen gewährleistet die korrekte Behandlung der mehrfach auftretenden Stützstellen und Auswertungsstellen. ABSCL wurde mit Rücksicht auf die Partitionierung des Auswertungsgitters parallelisiert. Für die Interpolation auf Grundlage radialer Basisfunktionen müssen zwei Matrizen aufgestellt werden. Die eine basiert nur auf der Lage der Stützstellen und wird zur Bestimmung eines Koeffizientenvektors invertiert. Die interpo- 
lierten Werte an den Auswertungspunkten werden durch Multiplikation der zweiten aufzustellenden Matrix, basierend auf der Lage der Stützstellen und Auswertungsstellen, mit dem vorher berechneten Koeffizientenvektor erhalten. Die Matrizen werden im Folgenden auch mit Stützstellen-Matrix und Auswertungsstellen-Matrix bezeichnet. Die Invertierung der Matrix wird auf Grundlage der akkumulierten Knotenkoordinaten der Stützstellen auf jedem Auswertungsgitter-Prozess vorgenommen. Die Matrizen zur Bestimmung der Werte auf den Auswertungsstellen werden parallel nur auf Grundlage der Auswertungsstellen der jeweiligen Partition aufgestellt.

Die Datenstrukturen in ABSCL wurden den Datenstrukturen in MpCCI angepasst, um zusätzlichen Zeitverlust infolge von Umkopieraktionen zu vermeiden. Da die an MpCCI gelieferten Koordinaten und Vektoren im Allgemeinen nicht auf Grundlage des gleichen Koordinatensystems spezifiziert werden, war der Einbau von Koordinatensystem-Transformationen notwendig.

Die Datenstrukturen wurden so verändert, dass eine im nächsten Schritt folgende Ausweitung der Interpolationsalgorithmen von dreidimensionalen Räumen auf $n$-dimensionale ermöglicht wurde. Im hier interessierenden Kopplungszusammenhang sind sicherlich höherdimensionale Räume von Interesse, wenn eine Interpolation über die Zeit notwendig wird. In anderen Kontexten, z.B. bei Interpolationen zwischen Simulationsläufen mit $n$ Parametern, werden Interpolationen dieser Art unumgänglich.

Das zunächst einfache Suchverfahren nach identischen Knoten an Partitionsgrenzen oder auch innerhalb der Partitionen wurde durch eine Suche mittels der Open Source Bibliothek „ANN“ [9] ersetzt, welche über „kd-trees“ und „,box-decomposition trees“ sucht.

Die gleiche Bibliothek ersetzt auch Teile der vorher rechenzeitintensiven Träger-Radius Bestimmung. Die in der Suche nach identischen Punkten erstellten Suchbäume werden hier zum zweiten Mal genutzt.

In ABSCL waren die radialen Basisfunktionen bislang nur mit polynomialen Termen bis zu linearer Ordnung implementiert. Um auch Funktionen mit quadratischer Ordnung exakt reproduzieren zu können, wurden zusätzlich polynomiale Terme zweiter Ordnung implementiert.

Ein Problem der Interpolationen auf Grundlage radialer Basisfunktionen, so wie sie in ABSCL implementiert waren, war der hohe Rechenzeit- und Speicherplatzaufwand, besonders bei einer großen Anzahl von Stützstellen. Die Matrizen wurden komplett aufgestellt, d.h. eine Matrix der Größe Stützstellenanzahl mal Stützstellenanzahl wurde mit einem direkten Verfahren invertiert. So konnte z.B. eine Interpolation von einem zur Verfügung gestellten „A340-300" Strukturgitter auf ein entsprechendes Strömungsgitter mit den verfügbaren Rechnern nur durchgeführt werden, wenn das Modell in kleinere Teile aufgesplittet wurde und diese einzeln interpoliert wurden. Bei einer solchen Methode geht Information für die Interpolation verloren und an den Übergängen entstehen nicht differenzierbare Stellen. Es wurden daher neue Verfahren benötigt, die mit großen Stützstellenanzahlen arbeiten können. 
Eine Methode, die von Wendland entwickelt wurde, beruht darauf, das „Partition of Unity“ Verfahren bei den radialen Basisfunktionen anzuwenden [10]. Hier wird so vorgegangen, dass das gesamte Gebiet, in dem die Interpolationen Anwendung finden sollen, mit vielen kleinen, überlappenden Gebieten überdeckt wird. In jedem der kleinen Gebiete werden die lokalen Interpolanten für alle darin liegenden Auswertungsstellen über radiale Basisfunktionen aufgestellt, auf Grundlage der Stützstellen, die in dem selben kleinen Gebiet liegen. Für jedes dieser Gebiete wird eine stetige Gewichtsfunktion definiert, die nur den Punkten innerhalb des Gebietes einen Wert ungleich Null zuordnet. Der Funktionswert für einen Punkt innerhalb des Gebietes ist so definiert, dass sich bei Addition aller Funktionswerte dieses Punktes bezüglich der Funktionen aller Gebiete immer eine Eins ergibt. Da der Punkt nur in den Gebieten, innerhalb denen er liegt, einen von Null verschiedenen Funktionswert hat, sind auch nur die Funktionen dieser Gebiete relevant für die Summe. Der globale Interpolationswert an einem Punkt resultiert dann aus der Summe der lokalen Interpolanten, multipliziert mit den jeweils dazugehörigen Funktionswerten über alle Gebiete innerhalb denen der Punkt liegt.

Ein Vorteil dieses Verfahrens ist, dass nur kleine Matrizen aufgestellt und invertiert werden müssen. Der Speicherplatz- und Rechenzeitbedarf kann so ganz erheblich gesenkt werden, wobei das Verfahren die Fehlerordnung gegenüber der Anwendung der Interpolation über das ganze Gebiet nicht verschlechtert. Ein entscheidendes Problem bei diesem Verfahren ist, eine geeignete Überdeckung mit kleinen Gebieten zu finden, die allen Bedingungen der Methode genügt und softwaretechnisch auch performant realisierbar ist.

Ein neuer Ansatz, der in dieser Arbeit entwickelt wurde, liefert sehr gute Resultate, was durch diverse Tests mit analytischen Funktionen belegt werden kann. Die Stützstellen sind bei diesen Beispielen auf komplexe Strukturen verteilt, wodurch sich sehr unregelmäßige Zellen ergeben. Für den zweidimensionalen Fall wird der neu entwickelte Algorithmus stark vereinfacht im Folgenden skizziert:

Die Koordinaten der Stützstellen seien mit $x$ und $y$ bezeichnet. Die Stützstellen werden zunächst nach der $x$-Koordinate sortiert. Dann werden die Stützstellen an Hand dieser Sortierung in $N_{1}$ möglichst gleich große Mengen geteilt. Von diesen $N_{1}$ Stützstellen-Mengen werden, angefangen mit der nach der Sortierung ersten Menge, immer $M$ aufeinanderfolgende Mengen zusammengenommen. Die $x$-Koordinate der ersten Stützstelle einer Menge und die $x$-Koordinate der auf die letzte Stützstelle einer Menge folgende Stützstelle, sofern diese existiert, definieren die Zellengrenzen in $x$-Koordinatenrichtung. Existiert diese Stützstelle nicht, so wird die $x$-Koordinate der letzten Stützstelle der Menge verwendet. Jede der entstandenen Mengen wird dann in $y$-Koordinatenrichtung sortiert, und es werden wieder an Hand dieser Sortierung $N_{2}$ Stützstellen-Mengen in jeder der vorherigen Mengen gebildet. Im Folgenden werden wieder auf die gleiche Art wie vorher $M$ Mengen zusammengefügt. Ebenso werden die erste Stützstelle einer Menge und, sofern 
sie existiert, die auf die letzte Stützstelle einer Menge folgende Stützstelle zur Bestimmung der Zellengrenzen in $y$-Koordinatenrichtung betrachtet. Bei der jeweils letzten Menge in einer Aufteilung der ersten $N_{1}$ StützstellenMengen muss wiederum die $y$-Koordinate der letzten Stützstelle der Menge zur Definition der Zellengrenzen verwendet werden. Der gesamte Algorithmus wird dann, angefangen mit der $y$-Koordinate, wiederholt. Die sich aus beiden Algorithmen ergebenden Rechtecke werden als die sich überlappenden Gebiete für die „Partition of Unity“ Methode kombiniert mit radialen Basisfunktionen verwendet. Der Algorithmus ist für $n$-dimensionale Räume implementiert. Durch mehrere Konfigurationsparameter kann in den Algorithmus eingegriffen werden. So kann z.B. auch bei einem dreidimensionalen Raum die Aufteilung nach nur zwei Dimensionen erfolgen. Die Mengengrößen, Überlappbereichs-Anzahlen, Sortier-Reihenfolge der Koordinatenrichtungen usw. sind ebenfalls, abweichend von einer Defaulteinstellung, bestimmbar.

Ein Algorithmus dieser Art ist bisher nicht in der Literatur zu finden und stellt auch in Verbindung mit der „Partition of Unity“ Methode bei radialen Basisfunktionen einen neuen Ansatz dar.

Bei einer bestimmten Konstellation von Stützstellen und Auswertungsstellen, verbunden mit einer $\mathrm{zu}$ interpolierenden analytischen Funktion, ergaben sich in Teilbereichen der Auswertungsstellen zu starke Abweichungen von der zu erreichenden Funktion. Diese Teilbereiche liegen in einem Gebiet, in dem schon extrapoliert werden muss, da in einer Richtung in direkter Nähe keine Stützstellen existieren. Da eine schlechtere Rekonstruktion in Extrapolationsbereichen durch die Theorie begründet ist, ist hier kein Problem in dem Verfahren oder der Implementierung zu sehen.

Um ein globales Vergleichsverfahren an der Hand zu haben, welches die radialen Basisfunktionen auch bei einer großen Anzahl von Stützstellen, wie es in der oben beschriebenen Konstellation der Fall ist, noch global zur Interpolation verwenden kann, wurde ein weiterer Algorithmus implementiert. Da die Matrix, die sich bei einer großen Anzahl von Stützstellen ergibt, aus Speicherplatzgründen nicht mehr komplett aufzustellen ist und daher nicht mehr mit dem implementierten Gauß-Algorithmus invertiert werden kann, wurde ein iteratives Verfahren implementiert, für welches die Matrix zur Lösung eines Gleichungssystems nicht explizit aufgestellt werden muss und das außerdem die dünn besetzte Struktur der Matrizen auf Grundlage der radialen Basisfunktionen mit kompaktem Träger, wie Euklidischer Hut Funktion und Wendland 1-3 Funktion ausnutzen kann.

Es wurden zwei verschiedene Verfahren zur iterativen Lösung des Gleichungssystems ausgewählt, um die Resultate mit denen aus der „Partition of Unity" Methode kombiniert mit radialen Basisfunktionen auch hinsichtlich Speicherplatz- und Rechenzeitbedarf zu testen.

Das erste Verfahren ist ein BiCGStab Algorithmus, der auf Grundlage der Veröffentlichung [11] in der Interpolationsbibliothek implementiert wurde. Das Interpolationsverfahren mit BiCGStab ist auf drei verschiedene Arten programmiert worden: 
1. Die Stützstellen-Matrix und die Auswertungsstellen-Matrix werden explizit in einer „Sparse-Matrix-Form” in einer Routine vor der eigentlichen Lösungsphase aufgestellt.

2. Es wird nur die Stützstellen-Matrix explizit in einer „Sparse-MatrixForm" in einer Routine vor der eigentlichen Lösungsphase aufgestellt. Die Auswertungsstellen-Matrix wird in jedem Schritt neu berechnet, in dem sie benötigt wird.

3. Beide verwendeten Matrizen werden in jedem Schritt neu berechnet, in dem sie benötigt werden.

Im zweiten Verfahren wurde der in SCAI entwickelte kommerzielle Algebraic Multigrid Solver AMG (siehe [12]) als Bibliothek an die Interpolation angeschlossen.

Für die iterativen Verfahren musste die Schnittstelle zu MpCCI erweitert werden. Bisher konnte die Kopplungsmatrix, aufgesplittet in die Matrizen der Paare von Sender- und Empfängerpartitionen, vor der eigentlichen Kopplungsphase an MpCCI spezifiziert werden. Dann konnte MpCCI in jedem Kopplungsschritt mit diesen Matrizen arbeiten. Da aber bei den iterativen Verfahren keine invertierte Matrix resultiert, sondern das Gleichungssystem mit rechter Seite gelöst wird, muss bei jedem Senden und Empfangen eines Prozesses eingegriffen und der Lösungsalgorithmus gestartet werden. So ist eine Schnittstelle implementiert worden, die es ermöglicht, eine Funktion für Sender- und Empfängerprozess zu schreiben. Diese Funktionen werden dann jeweils beim Versenden und Empfangen der Daten aufgerufen. Die Funktion, die auf jedem Senderprozess aufgerufen wird, erhält die Kopplungsdaten für jedes relevante Gitter sowie jede Partition des Senderprozesses und definiert die über MPI an jedes Empfängergitter, jeden Empfängerprozess und jede Empfängerpartition zu versendenden Daten. Die versendeten Daten werden der Funktion, die auf jedem Empfängerprozess aufgerufen wird, für jedes relevante Empfängergitter und jede Empfängerpartition übergeben. Die Werte für das spezifizierte Empfängergitter sind entsprechend der jeweiligen Partition des Prozesses zu definieren.

Die Vergleiche zeigten, dass ähnliche Probleme wie bei der „Partition of Unity“ Methode kombiniert mit radialen Basisfunktionen bei einem zu klein gewählten Träger-Radius auftauchten. Daher lag die Vermutung nahe, dass bei der „Partition of Unity“ Implementierung die Zellen im kritischen Bereich größer gesetzt werden sollten, wiewohl ihre Größe in anderen Bereichen absolut für sehr gute Ergebnisse ausreichte. So wurde eine automatische Vergrößerung der Partitionen in kritischen Bereichen implementiert. Mit diesem Verfahren oder generell sehr großen Partitionen wurden die besten Resultate mit den geringsten Fehlern erzielt.

Durch die Implementierung der Vergleichsverfahren konnten auch Vergleiche der Rechenzeiten bei großen Problemen durchgeführt werden. Die Rechenzeiten sind bei der „Partition of Unity“ Methode kombiniert mit radialen Basisfunktionen wesentlich besser als bei beiden iterativen Verfahren, 
die zur Verwendung kamen. Hinsichtlich des Speicherplatzbedarfs ist nur die dritte der oben aufgeführten Implementierungen des BiCGStab der „Partition of Unity" Methode kombiniert mit radialen Basisfunktionen vorzuziehen, da hier in jedem Schritt alle Werte neu berechnet werden und so nur die Ausgangsinformationen im Speicher gehalten werden müssen.

Es sollte noch erwähnt werden, dass die iterativen Verfahren für eine gekoppelte Simulation in der Aeroelastik mit der bisherigen Realisierung der Erhaltungssätze durch die Transformation der Kräfte über die transponierte Matrix nicht eingesetzt werden können. Die iterativen Algorithmen liefern keine invertierte Matrix, die multipliziert mit der Auswertungsstellen-Matrix die Kopplungsmatrix zur Transformation der Verschiebungen darstellt; so steht für die Transformation der Kräfte keine Matrix zur Verfügung, die transponiert werden kann. Nur durch diese transponierte Matrix wird aber bisher die Konservativität bei der Interpolation der Kräfte und die Erhaltung der Arbeit in der Kopplung gewährleistet. Außerdem ist zu bedenken, dass diese Verfahren in jedem Kopplungsschritt, auch bei unveränderten Gittern, neu gestartet werden müssen.

Im nächsten Schritt wurden neue Verfahren zur Realisierung der Zusatzbedingungen speziell für die Strömung-Struktur-Kopplung entwickelt, implementiert, an Beispielen getestet und mit den bisherigen Verfahren verglichen. Die Motivation für die Entwicklung neuer Verfahren lag zum Teil in theoretischen Überlegungen und zum Teil in praktischen Erfahrungen mit den bisherigen Verfahren.

In einigen der in der Vergangenheit durchgeführten Kopplungen mit den bisherigen Verfahren zeigte sich, dass die Transformation der Kräfte zu großen Unterschieden bei Verwendung verschiedener radialer Basisfunktionen geführt hat. Gerade bei radialen Basisfunktionen, die für die Deformationen sehr gute Resultate lieferten, zeigten dann die transformierten Kräfte bei einigen Anwendungen Verläufe, die keine Ähnlichkeiten zu denen der Ausgangswerte zeigten. Die Kräfte wurden in den alten Verfahren über die Transponierte der Kopplungsmatrix transformiert, die auf Grundlage radialer Basisfunktionen zur Interpolation der Deformationen entstanden ist. So ist die Transformation der Deformationen und der Kräfte an ein und die selbe radiale Basisfunktion gebunden. Für die Transformation der Kräfte wird also keine Interpolation angewendet, sondern eine Verteilung der Daten unter Einhaltung von Erhaltungssätzen realisiert. Um hier über alternative Verfahren verfügen zu können, sind Konzepte erarbeitet und implementiert worden, bei denen Interpolationen auf Grundlage radialer Basisfunktionen durchgeführt werden, die als Zusatzbedingungen die Erhaltungssätze, wie die Konservativität und die Erhaltung der Arbeit, realisieren.

Bei diesen neu implementierten Algorithmen über Zusatzbedingungen kann für die Verschiebungen eine andere radiale Basisfunktion verwendet werden als zur Interpolation der Kräfte. Nun kann z.B. die Wendland 3 Funktion für die Interpolation der Deformationen und die Volume Spline Funktion für die Kräfte als Grundlage dienen, wenn z.B. Wendland 3 für die Verschiebun- 
gen sehr gute Ergebnisse liefert, bei der Interpolation der Kräfte aber weniger sinnvolle Verläufe zeigt als Volume Spline. Dies ist bei der Verwendung der Transponierten nicht möglich, da schon die Interpolation der Verschiebungen die radiale Basisfunktion festlegt.

In den bisherigen Interpolationen mit radialen Basisfunktionen werden Rotationswerte, sofern vorhanden, nicht als Information verwendet. Diese Informationen gehen bei den Interpolationen mit radialen Basisfunktionen, im Gegensatz zu denen mit finiten Interpolationselementen, verloren. Um auch auf Grundlage radialer Basisfunktionen in den Interpolationen die gegebenen Informationen möglichst optimal auszunutzen, sind auch hier neue Algorithmen entwickelt und implementiert worden, die die Rotationen in die Interpolationen der Deformationen mit einbeziehen. Bei dieser Interpolation werden die Komponenten nicht mehr einzeln interpoliert, sondern die Deformations- und Rotationskomponenten sind untereinander in der Interpolation abhängig. Die Interpolationsmatrizen sind dementsprechend 36 mal so groß, wie die Matrizen für die komponentenweisen Interpolationen.

Da alle neu entwickelten Algorithmen zur Interpolation mit speziellen Zusatzbedingungen auch auf die bereits oben angesprochenen großen Probleme angewendet werden sollten, wurden diese Interpolationen ebenfalls mit der „Partition of Unity“ Methode kombiniert. Hier mussten die lokalen Matrizen auf Grund der zusätzlichen Gewichtsfunktion verändert werden, um die Bedingungen global zu erfüllen.

Im Zuge der Entwicklung dieser verschiedenen neuen Implementationen wurde ein Redesign der Interpolationsbibliothek unumgänglich. Die Schnittstelle zu der Interpolationsbibliothek wurde aber nur insoweit verändert, als zusätzliche Funktionen verfügbar sind.

Die Interpolationsbibliothek wird am Fraunhofer-Institut SCAI nicht nur in der multidisziplinären Simulation angewandt, sondern z.B. auch zur Darstellung einer von mehreren Parametern abhängigen Zielfunktion. In diesem Fall sind Werte der Zielfunktion von verschiedenen Parameterläufen gegeben. Durch die Interpolation auf ein gleichmäßiges Gitter von Auswertungsstellen kann dabei eine bessere Repräsentation der Zielfunktion gewonnen werden. Da die betrachteten Zielfunktionen durch sehr viele Peaks gekennzeichnet waren und so einen wenig glatten Verlauf zeigten, wurde für diese speziellen Anwendungen ein Approximationsverfahren implementiert. Mit diesem Approximationsverfahren werden die lokalen Minima und Maxima geglättet, bzw. verschwinden komplett, je nach Konfiguration der Approximation. Das Approximationsverfahren wurde ebenso wie die anderen Verfahren für große Probleme mit der „Partition of Unity" Methode kombiniert. In dieser Arbeit wird aber nur auf die Algorithmen, Implementierungen und Anwendungen im Bereich der multidisziplinären Simulation eingegangen.

Zur dateibasierten Ein- und Ausgabe sind Funktionen implementiert worden, die unter Verwendung der NetCDF-Bibliothek (siehe [13]) die Dateien im NetCDF-Format lesen und schreiben können. Damit können die gesamten Gitterinformationen, die zu interpolierenden Werte und die Kopplungsmatri- 
zen aus NetCDF-Dateien eingelesen werden und in NetCDF-Dateien abgelegt werden. Darüber hinaus ist die Ausgabe der Ergebnisse der Interpolationen in NetCDF-Dateien möglich.

Der letzte durchgeführte größere Arbeitsschritt betraf wieder die Performance der Interpolationen mit radialen Basisfunktionen. Da der größte Anteil der Rechenzeit für die Invertierung der Matrix verbraucht wird, wurden an dieser Stelle noch einmal einige Routinen verbessert. Das Gaußverfahren zur Invertierung der Matrix kann nun optional durch eine optimierte Routine aus der LAPACK-Bibliothek (Linear Algebra Package, siehe [14]) ersetzt werden, und an den Stellen, wo die invertierte Matrix nicht mehrmals benutzt werden kann, wird keine Invertierung mehr vorgenommen, sondern das Gleichungssystem mit rechter Seite direkt über ein LR-Verfahren gelöst.

Alle derzeit verfügbaren Verfahren sind auf eine umfangreiche Testsuite mit unterschiedlichen Strukturen und zugeordneten Werten aus analytischen Funktionen und Simulationsrechnungen angewendet worden. Die Abschlusstests bilden die Rechnungen, bei denen komplette Kopplungsläufe mit ausgewählten, neuen Interpolationsverfahren an realen Konfigurationen durchgeführt wurden.

Beschreibung der Kapitel. Das folgende Kapitel II enthält die Theorie zu den entwickelten neuen Algorithmen zur Interpolation auf der Grundlage radialer Basisfunktionen. Es führt die mathematischen Grundlagen ein und beschreibt detailliert die neu entwickelten Algorithmen. Im ersten Abschnitt wird eine kurze Einführung zu radialen Basisfunktionen gegeben. Der Abschnitt ist nicht im Sinne einer allgemeinen Einführung der radialen Basisfunktionen zu verstehen, sondern fokussiert nur auf die Informationen, die im Weiteren benötigt werden. Der darauf folgende Abschnitt beschreibt die Herleitung der neuen Algorithmen zur Realisierung von Zusatzbedingungen. Die Vorstellung der Verfahren zur Verbesserung der Rechenzeit und Speicherplatzanforderungen, und zwar auch in Kombination mit den Zusatzbedingungen, ist mit den erforderlichen Beweisen in Abschnitt $3 \mathrm{zu}$ finden. Teile der Abschnitte 1 und 3 sind aus den Arbeiten [4] und [10] von Wendland, sowie [15] von Schaback vom Institut für Numerische und Angewandte Mathematik der Georg-August-Universität Göttingen entstanden.

Im Kapitel III über die softwaretechnische Realisierung werden zunächst die für diese Arbeit relevanten Prozeduren von MpCCI beschrieben. Der Aufbau der Kopplungsbibliothek, der Ablauf einer gekoppelten Simulation und die Standard-Interpolationsalgorithmen aus MpCCI werden präsentiert. Im Anschluss daran werden die implementierten Funktionen zur Einbindung von speziellen Interpolationsalgorithmen in MpCCI beschrieben. Der Abschnitt zur Interpolationsbibliothek beginnt mit den Argumenten, die die Notwendigkeit der Verwendung spezieller Interpolationsalgorithmen für die Aeroelastik zeigen. Diese Motivation für die Anwendung von Interpolationen auf Grundlage radialer Basisfunktionen wird erst an dieser Stelle eingefügt, da die Begründungen auf den vorherigen theoretischen Erklärungen zu radialen 
Basisfunktonen sowie zu den Standard-Interpolationsalgorithmen in MpCCI aufbauen. In der Fortsetzung des Abschnitts wird auf die verwendeten softwaretechnischen Verfahren der Interpolationsbibliothek eingegangen. An dieser Stelle wird auch die Schnittstelle zwischen MpCCI und der Interpolationsbibliothek im Hinblick auf Parallelisierung und Datenhaltung erklärt.

Die Testbeispiele und Anwendungen, anhand derer die verschiedenen neuen Interpolationsalgorithmen demonstriert werden, sind in Kapitel IV zu finden. Gerechnete Beispiele werden präsentiert und erklärt. Die Testbeispiele zeigen der Reihe nach die Anwendung der einzelnen Algorithmen. Zusatzbedingungen werden zusammengestellt und an einem Beispiel dargelegt. Da hier nur die verschiedenen räumlichen Interpolationsverfahren gezeigt werden sollen, werden die Routinen an analytischen Funktionen und Ergebnissen aus bereits durchgeführten, gekoppelten Simulationsläufen demonstriert. Die Anwendungen werden nur in Hinblick auf ihre Funktion als Demonstrationsbeispiel erklärt. Die Beispiele, die nicht aus der Aeroelastik stammen, werden kurz beschrieben, um ein Verständnis der Anwendung zu ermöglichen. Der letzte Teil enthält die Resultate einer kompletten gekoppelten Simulation über MpCCI mit ausgewählten Routinen aus der Interpolationsbibliothek.

In der Zusammenfassung werden die neuen Entwicklungen noch einmal genannt und die entstandenen Verbesserungen im Hinblick auf die gekoppelte Simulation erläutert. Die durch die Interpolationsbibliothek gewonnenen neuen Möglichkeiten werden diskutiert. Der Ausblick enthält die Verfahren, die in diesem Kontext in Zukunft von Interesse sein könnten, und es werden bereits erarbeitete Konzepte vorgestellt. 


\section{Interpolationen mit radialen Basisfunktionen}

\section{Einführung}

Problembeschreibung. In numerischen Simulationen vieler naturwissenschaftlicher und technischer Verfahren wird in der Regel nicht auf kontinuierlichen sondern auf diskreten Mengen gearbeitet. Dies bedeutet, dass Daten im betrachteten Definitionsbereich nicht durch eine kontinuierliche Funktion gegeben sind, sondern i.a. in Form einer Tabelle von Werten, wobei jeder Wert einem bestimmten Punkt des Definitionsbereichs zugeordnet ist. Da aber vielfach der Wunsch oder auch die Notwendigkeit besteht, Werte an nicht explizit gegebenen Stellen zu betrachten, entsteht hier ein sogenanntes Rekonstruktionsproblem. Genauer gesagt ist das Problem die Rekonstruktion einer Funktion $f$ aus einer Menge von diskret gegebenen Daten. Diese Rekonstruktion kann auf zweierlei Arten durchgeführt werden:

1. Bei der Interpolation wird eine Funktion $s_{f, X}$ aus einer hinreichend großen Menge $\mathcal{F}$ von Funktionen gesucht, die die vorgegebenen Werte exakt reproduziert.

2. Bei der Approximation wird eine Funktion $s_{f, X}$ aus einer meist kleineren Menge $\mathcal{F}$ von Funktionen gewählt, die oft keine Interpolation mehr erlaubt. Dann tritt an den Daten zwangsläufig ein Fehler auf; es wird aber oft eine zusätzliche Eigenschaft, wie z.B. minimale Krümmung, garantiert.

Die Wahl zwischen Interpolation und Approximation ist abhängig von der Anwendung. Aus dem Wissen über die Anwendung muss entschieden werden, aus welcher Menge von Funktionen die Rekonstruktionsfunktion sein soll und wie wichtig eine exakte Reproduktion der Werte ist. Im allgemeinen sollten Approximationen bei fehlerhaften Daten, wie z.B. bei Messwerten angewendet werden.

In den gekoppelten Simulationen interessiert i.a. die Interpolation $d$-variater Funktionen $f$, die auf einem Gebiet $\Omega$ definiert sind. In den meisten Kopplungsanwendungen ist $\Omega$ eine Untermenge des $\mathbb{R}^{3}$. Die hier gegebenen Resultate gelten aber in fast allen Fällen für Untermengen des $\mathbb{R}^{d}$ und in vielen Fällen für allgemeine Mengen. Gegeben sind immer eine endliche Menge $X=\left\{x_{1}, \ldots, x_{N}\right\} \subseteq \Omega \subseteq \mathbb{R}^{d}$ von $N$ möglicherweise weit gestreuten Punkten, im Weiteren auch Stützstellen genannt, in einem Gebiet $\Omega \subseteq \mathbb{R}^{d}$, und reelle Werte $f_{1}, \ldots, f_{N}$, welche die Werte von $f$ an den gegebenen Punkten darstellen. Die Rekonstruktion sollte eine Funktion $s_{f, X}: \Omega \rightarrow \mathbb{R}$ bereitstellen, welche die Werte $f_{i}=f\left(x_{i}\right), 1 \leq i \leq N$ reproduziert, d.h. die

$$
s_{f, X}\left(x_{i}\right)=f_{i}, \quad 1 \leq i \leq N
$$


erfüllt. In einigen Fällen werden auch vektorwertige Funktionen betrachtet. Dann stammen die Werte $f\left(x_{j}\right)$ aus einem $\mathbb{R}^{n}$, d.h. $f$ ist eine Funktion, die $\Omega$ nach $\mathbb{R}^{n}$ abbildet, wobei hier in der Regel $n=d=3$ gilt. Im einfachsten Fall lassen sich die einzelnen Komponenten von $f$ unabhängig von einander interpolieren oder approximieren. Dazu muss dann nur eine Interpolationsmatrix aufgestellt werden, die dann auf die jeweiligen Komponentenvektoren angewendet wird.

Im Folgenden wird davon ausgegangen, dass das Gebiet $\Omega \subseteq \mathbb{R}^{d}$ gegeben ist. Ferner soll $\mathcal{F}$ ein gegebener linearer Raum von Funktionen $f: \Omega \rightarrow \mathbb{R}$ sein. Die genaue Wahl von $\Omega$ und $\mathcal{F}$ hängt vom jeweiligen Anwendungsproblem ab.

In Abhängigkeit von der Stützstellenmenge $X$ kann nun folgendes Problem auftreten. Gibt es eine Funktion $h \in \mathcal{F}$, die auf $X$ verschwindet, so ist neben einer Interpolante $s_{f, X}$ auch jede Funktion $s_{f, X}+\alpha h$ mit $\alpha \in \mathbb{R}$ Interpolante. Das Interpolationsproblem ist dann nicht eindeutig lösbar und es ist mit numerischen Problemen zu rechnen.

Definition 1.1 Sei $\mathcal{F}$ ein Vektorraum von Funktionen auf einem Gebiet $\Omega$. Eine Menge $X$ auf $\Omega$ wird als $\mathcal{F}$-nicht degeneriert oder $\mathcal{F}$-unisolvent bezeichnet, wenn die Null-Funktion die einzige Funktion von $\mathcal{F}$ ist, die auf allen Punkten von $X$ verschwindet.

Wie oben zu sehen war, können nur $\mathcal{F}$-nicht degenerierte Untermengen $X$ von $\Omega$ für eine numerisch stabile Rekonstruktion verwendet werden. Dies wäre unproblematisch, wenn jede endliche Menge $X$ oder, zumindest für einen gegebenen Vektorraum $\mathcal{F}$ mit $\operatorname{dim} \mathcal{F}=N$, jede Menge $X=\left\{x_{1}, \ldots, x_{N}\right\}$ nicht degeneriert wäre.

In echten multivariaten Situationen ist dies aber nicht der Fall. Wenn ein linearer Unterraum $\mathcal{F}$ mit Dimension $N \geq 2$ eines Vektorraumes von multivariaten Funktionen unabhängig von der Menge $X=\left\{x_{1}, \ldots, x_{N}\right\}$ festgelegt wird, existiert immer eine degenerierte Menge $X$. Diese Beobachtung wurde zuerst von Mairhuber und Curtis gemacht (für einen Beweis siehe [4]).

Theorem 1.2 (Mairhuber-Curtis) Sei $\mathcal{F}$ ein $N$-dimensionaler Vektorraum von stetigen reellwertigen Funktionen auf einem Gebiet $\Omega \subseteq \mathbb{R}^{d}$, welches in der Tat d-dimensional ist, in dem Sinne, dass $\Omega$ mindestens eine offene Untermenge $\Omega_{1}$ des $\mathbb{R}^{d}$ enthält. Wird weiter angenommen, dass jede Menge $X=\left\{x_{1}, \ldots, x_{N}\right\} \subseteq \Omega$ nicht degeneriert ist, dann gilt entweder $N=1$ oder $d=1$, das heißt, entweder der Vektorraum der Funktionen oder das darrunterliegende Gebiet ist eindimensional.

Als Folge des Mairhuber-Curtis-Theorems muss der gewählte Vektorraum $\mathcal{F}$ von den Daten abhängen. Wird vorausgesetzt, dass für gegebenes $X=$ $\left\{x_{1}, \ldots, x_{N}\right\}$ die Rekonstruktion linear von den Funktionswerten abhängt, ist es sinnvoll, $\mathcal{F}$ auch nur von den Datenorten $X=\left\{x_{1}, \ldots, x_{N}\right\}$ abhängen zu lassen und nicht von den Datenwerten $f_{1}, \ldots, f_{N}$. Ferner sollte $\mathcal{F}$ ein 
linearer Raum sein. Der einfachste Weg, dies zu erreichen, ist, die Argumente $x \in \Omega$ und $x_{i} \in X$ in einer einzigen Funktion zu kombinieren,

$$
\Phi: \Omega \times \Omega \rightarrow \mathbb{R}
$$

und $\Phi\left(x, x_{i}\right)$ als eine abhängige Funktion der Variable $x$ aufzufassen. Superposition von solchen Funktionen resultiert dann in einem Vektorraum

$$
\mathcal{F}_{X, \Phi}:=\left\{\sum_{i=1}^{N} \alpha_{i} \Phi\left(x, x_{i}\right): \alpha_{i} \in \mathbb{R}\right\} .
$$

Wenn für $\mathcal{F}_{\Phi}=\bigcup_{X} \mathcal{F}_{X, \Phi}$, die Vereinigung aller Funktionen-Vektorräume $\mathcal{F}_{X, \Phi}$ mit variierenden Mengen $X \subseteq \mathbb{R}^{d}$, Translationsinvarianz gefordert wird, dann kann gezeigt werden, dass $\Phi$ selbst translationsinvariant sein muss:

$$
\Phi=\phi(x-y), \phi: \mathbb{R} \rightarrow \mathbb{R}
$$

Bei zusätzlicher Rotationsinvarianz muss $\Phi$ eine sogenannte radiale Basisfunktion sein:

$$
\Phi=\phi\left(\|x-y\|_{2}\right), \phi:[0, \infty) \rightarrow \mathbb{R}
$$

wobei $\|\cdot\|_{2}$ die gewöhnliche Euklidische Norm des $\mathbb{R}^{d}$ bezeichnet.

Definition 1.3 Eine Funktion $\Phi: \mathbb{R}^{d} \rightarrow \mathbb{R}$ heißt radial, wenn eine Funktion $\phi:[0, \infty) \rightarrow \mathbb{R}$ existiert, so dass $\Phi(x)=\phi\left(\|x\|_{2}\right)$ für alle $x \in \mathbb{R}^{d}$ gilt.

Eine beliebige Funktion aus dem Vektorraum (2.1) hat nun die Darstellung

$$
f_{\alpha}(x):=\sum_{j=1}^{N} \alpha_{j} \Phi\left(x, x_{j}\right), \quad x \in \Omega \subseteq \mathbb{R}^{d} .
$$

Der Koeffizientenvektor $\left(\alpha_{1}, \ldots, \alpha_{N}\right)$ muss also in Abhängigkeit des konkreten Rekonstruktionsproblems bestimmt werden.

Die Auswertung einer solchen Funktion für eine große Anzahl von verschiedenen Punkten $x \in \Omega$ kann sehr rechenzeitintensiv sein, falls $N$ ebenfalls sehr groß ist. Die starke Abhängigkeit der Rechenzeit von $N$ wird aber abgeschwächt bei Funktionen $\Phi\left(x, x_{i}\right)$, deren Wert null wird, wenn $x$ und $x_{i}$ weiter als ein gegebener Abstand auseinander liegen. Beispiele solcher Funktionen werden später gegeben.

Wird die Rekonstruktion durch Interpolation auf der Stützstellenmenge $X=\left\{x_{1}, \ldots, x_{N}\right\}$ bestimmt, so muss das lineare Gleichungssystem

$$
\sum_{j=1}^{N} \alpha_{j} \Phi\left(x_{i}, x_{j}\right)=f_{i}, \quad 1 \leq i \leq N
$$


gelöst werden. In Matrixschreibweise wird die Form

$$
A \alpha=f
$$

erhalten, bzw. wenn die Abhängigkeit von $X$ und $\Phi$ deutlich gemacht werden soll:

$$
A_{\Phi, X} \alpha_{\Phi, X}=f_{X}
$$

mit der Interpolationsmatrix

$$
A_{\Phi, X}=\left(\Phi\left(x_{i}, x_{j}\right)\right)_{1 \leq i, j \leq N}
$$

Damit das System eindeutig lösbar ist, muss die Matrix $A_{\Phi, X}$ nichtsingulär sein. Aus numerischer Sicht ist es sinnvoll sogar zu verlangen, dass die Matrix $A_{\Phi, X}$ positiv definit oder zumindest unter bestimmten Bedingungen positiv definit ist. Soll dieselbe Funktion $\Phi$ für alle möglichen Datensätze $X \subseteq \Omega$ benutzt werden, so muss dies auch für alle Matrizen $A_{\Phi, X}$ gelten.

Definition 1.4 Eine reellwertige Funktion

$$
\Phi: \Omega \times \Omega \rightarrow \mathbb{R}
$$

ist eine positiv definite Funktion auf $\Omega$, wenn für jede Wahl von endlichen Untermengen $X=\left\{x_{1}, \ldots, x_{N}\right\} \subseteq \Omega$ von $N$ verschiedenen Punkten die Matrix

$$
A_{\Phi, X}=\left(\Phi\left(x_{i}, x_{j}\right)\right)_{1 \leq i, j \leq N}
$$

positiv definit ist, d.h. wenn $A_{\Phi, X}$ symmetrisch ist und

$$
\alpha^{T} A_{\Phi, X} \alpha>0
$$

für alle $\alpha \in \mathbb{R}^{d} \backslash\{0\}$ erfüllt ist.

Dies bedeutet insbesondere, dass die Funktion $\Phi$ selbst symmetrisch sein muss, d.h. sie muss $\Phi(x, y)=\Phi(y, x)$ erfüllen, was bei radialen Funktionen immer automatisch der Fall ist. Ist $\Phi$ positiv definit auf $\Omega$, so kann durch

$$
(\Phi(\cdot, x), \Phi(\cdot, y))_{\Phi}:=\Phi(x, y)
$$

ein Skalarprodukt auf $\mathcal{F}=\operatorname{span}\{\Phi(\cdot, x): x \in \Omega\}$ definiert werden und $\mathcal{F}$ bzgl. dieses Skalarproduktes abgeschlossen werden, so dass $\mathcal{F}$ zu einem Hilbertraum von Funktionen wird. Details sind nachzulesen in [4].

Auch wenn es auf den ersten Blick erstaunlich ist, dass mit einer festen Funktion $\Phi$ alle Matrizen der oben angegebenen Form auf jeder gewählten Menge $X$ positiv definit sein sollen, so erfüllen einige radiale Basisfunktionen wie z.B. $\Phi\left(x, x_{i}\right)=\exp \left(-\left\|x-x_{i}\right\|_{2}^{2}\right)$ dies sogar für jede Raumdimension. 
Bedingt positiv definite radiale Basisfunktionen. Die folgenden Beispiele für radiale Basisfunktionen wurden in vielen Anwendungen erfolgreich verwendet:

- Die Thin-plate-spline-Funktion, die von Duchon [16] eingeführt wurde,

$$
\Phi(x, y)=\|x-y\|_{2}^{2} \log \|x-y\|_{2} .
$$

- Die Multiquadric-Funktion, die von Hardy in der Geophysik [17] verwendet wurde,

$$
\Phi(x, y)=\left(c^{2}+\|x-y\|_{2}^{2}\right)^{\frac{1}{2}}
$$

- Die inverse Multiquadric-Funktion, die ebenfalls von Hardy [17] verwendet wurde,

$$
\Phi(x, y)=\frac{1}{\left(c^{2}+\|x-y\|_{2}^{2}\right)^{\frac{1}{2}}} .
$$

Die ersten beiden Funktionen sind nicht positiv definit. Die dazugehörigen Matrizen $A_{\Phi, X}$ definieren aber quadratische Formen $Q_{X, \Phi}: \mathbb{R}^{N} \rightarrow \mathbb{R}$ durch

$$
Q_{X, \Phi}: \alpha=\left(\alpha_{1}, \ldots, \alpha_{N}\right) \mapsto \alpha^{T} A_{\Phi, X} \alpha:=\sum_{i, j=1}^{N} \alpha_{i} \alpha_{j} \Phi\left(x_{i}, x_{j}\right)
$$

die immerhin noch positiv definit auf einem geeigneten Teilraum von $\mathbb{R}^{N}$ sind. Für bestimmte $m \in \mathbb{N}$ erfüllen die oben angegebenen Funktionen $\Phi$ die folgende Definition:

Definition 1.5 Eine symmetrische, reellwertige Funktion $\Phi: \Omega \times \Omega \rightarrow \mathbb{R}$ ist eine bedingt positiv definite Funktion der Ordnung $m$ auf $\Omega \subseteq \mathbb{R}^{d}$, wenn für jede endliche Untermenge $X=\left\{x_{1}, \ldots, x_{N}\right\} \subseteq \Omega$ von $N$ verschiedenen Punkten der Wert der quadratischen Form

$$
\alpha^{T} A_{\Phi, X} \alpha:=\sum_{i, j=1}^{N} \alpha_{i} \alpha_{j} \Phi\left(x_{i}, x_{j}\right)
$$

positiv ist, vorausgesetzt der Vektor $\alpha=\left(\alpha_{1}, \cdots, \alpha_{N}\right) \in \mathbb{R}^{N} \backslash\{0\}$ hat die zusätzliche Eigenschaft

$$
\sum_{i=1}^{N} \alpha_{i} p\left(x_{i}\right)=0
$$

für alle d-variaten Polynome vom Grad $\leq m-1$. Der lineare Vektorraum von solchen Polynomen wird mit $\Pi_{m-1}\left(\mathbb{R}^{d}\right)$ bezeichnet. Die Dimension des Vektorraums ist

$$
Q:=\left(\begin{array}{c}
m-1+d \\
d
\end{array}\right)
$$


In [18] wird der Beweis zu der bedingten Positiv-Definitheit einer festen Ordnung $m$ der Multiquadric-Funktionen für alle Raumdimensionen $d$ geführt.

Bei der Interpolation mit bedingt positiv definiten Funktionen der Ordnung $m>0$ muss nun die Ansatzfunktion aus (2.2) leicht abgeändert werden. Dazu wird eine Basis $p_{1}, \ldots, p_{Q}$ von $\Pi_{m-1}\left(\mathbb{R}^{d}\right)$ gewählt und der Ansatz

$$
s_{f, X}(x)=\sum_{j=1}^{N} \alpha_{j} \Phi\left(x, x_{j}\right)+\sum_{k=1}^{Q} \beta_{k} p_{k}(x)
$$

gemacht. Die Koeffizientenvektoren $\alpha \in \mathbb{R}^{N}$ und $\beta \in \mathbb{R}^{Q}$ sind dann durch die Interpolationsbedingungen $s_{f, X}\left(x_{i}\right)=f\left(x_{i}\right), 1 \leq i \leq N$ und die zusätzlichen Bedingungen

$$
\sum_{i=1}^{N} \alpha_{j} p_{j}\left(x_{i}\right)=0, \quad 1 \leq j \leq Q
$$

zu bestimmen. Es gilt dabei:

Theorem 1.6 Ist die Funktion $\Phi: \Omega \times \Omega \rightarrow \mathbb{R}$ bedingt positiv definit der Ordnung $m>0$ und ist $X=\left\{x_{1}, \ldots, x_{N}\right\} \subseteq \Omega$ eine $\Pi_{m-1}\left(\mathbb{R}^{d}\right)$-unisolvente Teilmenge, so gibt es genau eine Interpolante der Form (2.5), die die zusätzlichen Bedingungen (2.6) erfüllt.

Wird neben der $N \times N$ Matrix $A_{\Phi, X}=\left(\Phi\left(x_{i}, x_{j}\right)\right)$ noch die Matrix $P_{X}:=\left(p_{j}\left(x_{i}\right)\right) \in \mathbb{R}^{N \times Q}$ eingeführt, so lässt sich das resultierende lineare Gleichungssystem schreiben als

$$
\left(\begin{array}{cc}
A_{\Phi, X} & P_{X} \\
P_{X}^{T} & 0
\end{array}\right)\left(\begin{array}{c}
\alpha \\
\beta
\end{array}\right)=\left(\begin{array}{c}
f \mid X \\
0
\end{array}\right)
$$

Werden dann für gegebene Auswertungsstellen $Y=\left\{y_{1} \ldots, y_{M}\right\} \subseteq \Omega$ zusätzliche Matrizen $A_{\Phi, X, Y}=\left(\Phi\left(y_{i}-x_{j}\right)\right) \in \mathbb{R}^{M \times N}$ und $P_{Y}=p_{j}\left(y_{i}\right) \in \mathbb{R}^{M \times Q}$ definiert, so lässt sich die lineare Abbildung $H: \mathbb{R}^{N} \rightarrow \mathbb{R}^{M}$, die die Interpolationswerte $f \mid X$ auf die interpolierten Werte $s_{f, X} \mid Y$ abbildet aus

$$
s_{f, X} \mid Y=\left(\begin{array}{ll}
A_{\Phi, X, Y} & P_{Y}
\end{array}\right)\left(\begin{array}{cc}
A_{\Phi, X} & P_{X} \\
P_{X}^{T} & 0
\end{array}\right)^{-1}\left(\begin{array}{c}
f \mid X \\
0
\end{array}\right)=:\left(\begin{array}{cc}
H & \widetilde{H}
\end{array}\right)\left(\begin{array}{c}
f \mid X \\
0
\end{array}\right)
$$

ablesen, wobei die dabei zusätzlich auftretende Matrix $\widetilde{H}$ die Dimensionen $M \times Q$ hat.

In den Tabellen 1 und 2 werden die wichtigsten (bedingt) positiv definiten radialen Basisfunktionen aufgelistet. In Tabelle 1 sind die bedingt positiv definiten Funktionen der Ordnung $m>0$ auf $\mathbb{R}^{d}$ angegeben, wobei $m$ sowie die in den Funktionen auftretenden Parameter näher spezifiziert werden. Diese Funktionen sind für jede beliebige Raumdimension anwendbar. Da sie mit $r$ gegen Unendlich wachsen, generieren sie voll besetzte Matrizen und sind 


\begin{tabular}{|c|c|c|}
\hline$\phi(r)$ & Parameter & Ordnung \\
\hline$r^{\beta}$ & $\beta>0, \beta \notin 2 \mathbb{N}$ & $m \geq\left\lceil\frac{\beta}{2}\right\rceil$ \\
$r^{\beta} \log r$ & $\beta>0, \beta \in 2 \mathbb{N}$ & $m>\frac{\beta}{2}$ \\
$\left(r^{2}+c^{2}\right)^{\frac{\beta}{2}}$ & $\beta>0, \beta \notin 2 \mathbb{N}$ & $m \geq\left\lceil\frac{\beta}{2}\right\rceil$ \\
\hline
\end{tabular}

Tabelle 1. Bedingt positiv definite radiale Basisfunktionen.

deshalb speziell gut für Anwendungen, in denen ein Abfall der Werte der Rekonstruktionsfunktion gegen Unendlich falsch wäre.

In der Tabelle 2 werden die positiv definiten radialen Basisfunktionen mit ihren Bezeichnungen aufgeführt. In dieser Tabelle sind die Glätte und die Bereiche, aus denen die Parameter in den Funktionen gewählt werden dürfen, spezifiziert. Darüber hinaus enthält diese Tabelle die Dimensionen, in denen die jeweilige radiale Basisfunktion als positiv definite Funktion gilt. Bei den Funktionen mit kompaktem Träger existiert keine Funktion, die für alle Raumdimensionen anwendbar ist, wie in [15] bewiesen wird. Funktionen mit kompaktem Träger liefern dünn besetzte Matrizen, aber die Wahl eines vernünftigen Trägerradius ist nicht einfach. Bei einem Radius, der zu klein ist, erhalten die Matrizen eine diagonal dominante Struktur, so dass die numerische Lösung sehr effizient und stabil wird, die Reproduktionsqualität wird allerdings inakzeptabel, da die Funktion nur an den Stützstellen „Peaks“ aufweist und auf einem engen Radius um die Stützstellen abfällt. Ein großer Trägerradius liefert dagegen eine gute Reproduktionsqualität. Die Matrizen sind umso voller besetzt, je größer der Trägerradius ist, so dass der Aufwand bei größer werdendem Trägerradius steigt. Dies ist auch eine Konsequenz der im Folgenden beschriebenen „Unschärferelation“.

Allgemeine Bemerkungen zu Interpolationen mit radialen Basisfunktionen. Die erwähnte „Unschärferelation“ lautet, bezogen auf die hier bearbeitete Problematik:

Wenn eine hohe Reproduktionsqualität benötigt wird, muss eine schlechte numerische Stabilität in Kauf genommen werden. Wenn eine gute numerische Qualität benötigt wird, muss eine schlechte Reproduktionsqualität in Kauf genommen werden.

Dies gilt nicht nur im Zusammenhang mit dem Radius einer radialen Basisfunktion mit kompaktem Träger; die Reproduktionsqualität und die numerische Stabilität sind verbunden mit der Datendichte und der Glätte der radialen Basisfunktion. Darüber hinaus ist bei der Lösung großer linearer Gleichungssysteme mit positiv definiten Matrizen z.B. durch ein konjugiertes Gradientenverfahren die numerische Stabilität direkt über die Kondition der Matrix mit der Effizienz verknüpft. Für große Probleme kann so Stabilität durch Effizienz in der Unschärferelation ersetzt werden. 


\begin{tabular}{|c||c|c|c|c|}
\hline$\phi(r)$ & Para- & Glätte & Dimen- & Name \\
meter & & sion & \\
\hline$e^{-\beta r^{2}}$ & $\beta>0$ & $C^{\infty}\left(\mathbb{R}^{d}\right)$ & $d<\infty$ & Gauß \\
$\left(r^{2}+c^{2}\right)^{\frac{\beta}{2}}$ & $\beta<0$ & $C^{\infty}\left(\mathbb{R}^{d}\right)$ & $d<\infty$ & inv. Multiquadrics \\
$r^{\nu} K_{\nu}(r)$ & $\nu>0$ & $C^{[\nu]}$ & $d<\infty$ & Sobolev Spline \\
$\pi\left(\left(\frac{1}{12} r^{3}\right)-\left(R^{2} r\right)+\left(\frac{4}{3} R^{3}\right)\right)$ & $r<2 R$ & $C^{0}$ & $d \leq 3$ & Euklid's Hut \\
$\left(1-r^{2}\right)_{+}^{2}(2+r)$ & & $C^{0}$ & $d \leq 3$ & Wu \\
$(1-r)_{+}$ & & $C^{0}$ & $d=1$ & Wendland \\
$(1-r)_{+}^{3}(3 r+1)$ & & $C^{2}$ & $d=1$ & Wendland \\
$(1-r)_{+}^{5}\left(8 r^{2}+5 r+1\right)$ & & $C^{4}$ & $d=1$ & Wendland \\
$(1-r)_{+}^{2}$ & & $C^{0}$ & $d \leq 3$ & Wendland \\
$(1-r)_{+}^{4}(4 r+1)$ & & $C^{2}$ & $d \leq 3$ & Wendland \\
$(1-r)_{+}^{6}\left(35 r^{2}+18 r+3\right)$ & & $C^{4}$ & $d \leq 3$ & Wendland \\
$(1-r)_{+}^{8}\left(32 r^{3}+25^{2} r+8 r+1\right)$ & & $C^{6}$ & $d \leq 3$ & Wendland \\
$(1-r)_{+}^{3}$ & & $C^{0}$ & $d \leq 5$ & Wendland \\
$(1-r)_{+}^{5}(5 r+1)$ & & $C^{2}$ & $d \leq 5$ & Wendland \\
$(1-r)_{+}^{7}\left(16 r^{2}+7 r+1\right)$ & & $C^{4}$ & $d \leq 5$ & Wendland \\
\hline
\end{tabular}

Tabelle 2. Positiv definite radiale Basisfunktionen.

Bei gleicher Datendichte ergibt sich aus der Unschärferelation, dass die Glätte so gering sein sollte, wie die Anwendung es zulässt, denn jede unnötig hohe Glätte bedingt wiederum eine schlechtere Stabilität.

Um eine gute Reproduktionsqualität und Stabilität zu erhalten, sollte die folgende Regel, die die Datendichte mit dem zu wählenden Trägerradius und der zu wählenden Glätte in Beziehung setzt, beachtet werden: Bei einer kleinen Datenmenge von dünn verteilten Daten sollte mit glatten Funktionen und größerem Träger gearbeitet werden. Bei einer großen Anzahl dicht liegender Stützstellen sollte der Trägerradius und eventuell auch die Glätte der Basisfunktion reduziert werden. So werden bei radialen Basisfunktionen mit kompaktem Träger Probleme bei sehr kleinen oder großen Datenmengen vermieden.

Die folgende Beobachtung wird für diese Arbeit von entscheidender Bedeutung sein. Radiale Basisfunktionen eignen sich nicht nur zur Rekonstruktion einer Funktion aus gegebenen Funktionswerten $f\left(x_{j}\right)$ einer Funktion $f$ an den Datenstellen $x_{j}$. Vielmehr lassen sich mit ihnen Werte $f_{j}=\lambda_{j}(f)$, die durch allgemeine Funktionale $\lambda_{j}$ gegeben werden, rekonstruieren. So könnten 
die $f_{j}$ z.B. Zellmittel einer unbekannten Funktion $f$ sein, d.h.

$$
f_{j}=\int_{V_{j}} f(x) d x /\left|V_{j}\right| .
$$

In dieser Arbeit werden Funktionale der Form

$$
\lambda(f)=\sum_{j=1}^{N} w_{j} f\left(x_{j}\right)
$$

auftreten, die die Erhaltung von Arbeit und Energie künstlich erzwingen. Ein solches verallgemeinertes Interpolationsproblem lässt sich also folgendermaßen beschreiben. Gegeben sind $N$ über $\mathcal{F}$ linear unabhängige Funktionale $\lambda_{1}, \ldots, \lambda_{N}$ und zugehörige Werte $f_{1}, \ldots, f_{N}$. Gesucht ist dann eine Funktion $s_{f, \Lambda} \in \mathcal{F}$, die die Interpolationsbedingungen

$$
\lambda_{j}\left(s_{f, \Lambda}\right)=f_{j}, \quad 1 \leq j \leq N
$$

erfüllt.

Um eine solche Interpolationsaufgabe zu lösen, muss der bisherige Ansatz verfeinert werden. Statt einen Ansatz der Form (2.2) zu machen, werden die Funktionale nun auch in den Ansatzraum mit eingebaut. Man setzt dann

$$
s_{f, \Lambda}(x)=\sum_{j=1}^{N} \alpha_{j} \lambda_{j}^{y} \Phi(x, y),
$$

wobei der obere Index andeuten soll, dass das Funktional auf $\Phi$ bzgl. der Variablen $y$ wirkt. Man beachte, dass bei der Wahl $\lambda_{j}(f):=f\left(x_{j}\right)$ der neue Ansatz mit dem alten übereinstimmt. Es gilt nun folgender Zusammenhang:

Theorem 1.7 Ist $\Phi: \Omega \times \Omega \rightarrow \mathbb{R}$ positiv definit, und sind die linearen Funktionale $\lambda_{1}, \ldots, \lambda_{N}$ über $\mathcal{F}$ linear unabhängig, so gibt es genau eine Funktion $s_{f, \Lambda} \in \mathcal{F}$ der Form (2.9), die die verallgemeinerten Interpolationsbedingungen (2.8) erfüllt.

Ein Beweis findet sich wieder in [4]. Die Koeffizienten $\alpha_{j}$ werden wieder durch Lösen des linearen Gleichungssystems bestimmt, das durch die Interpolationsbedingungen (2.8) entsteht. Auf Grund des Ansatzes (2.9) hat es die symmetrische Koeffizientenmatrix

$$
A_{\Phi, \Lambda}=\left(\lambda_{i}^{x} \lambda_{j}^{y} \Phi(x, y)\right)_{1 \leq i, j \leq N}
$$

die mit der Matrix in (2.3) im Fall von Punktauswertungsfunktionalen, also $\lambda_{j}(f)=f\left(x_{j}\right)$, übereinstimmt.

Ein ähnliches Resultat wie Theorem 1.7 gilt für den Fall bedingt positiv definiter Funktionen: 
Theorem 1.8 Ist $\Phi: \Omega \times \Omega \rightarrow \mathbb{R}$ bedingt positiv definit der Ordnung $m$, und sind die linearen Funktionale $\lambda_{1}, \ldots, \lambda_{N}$ linear unabhängig, so gibt es genau eine Funktion $s_{f, \Lambda}$ der Form

$$
s_{f, \Lambda}(x)=\sum_{j=1}^{N} \alpha_{j} \lambda_{j}^{y} \Phi(x, y)+\sum_{k=1}^{Q} \beta_{k} p_{k}(x),
$$

welche die verallgemeinerten Interpolationsbedingungen (2.8) und die Nebenbedingungen

$$
\sum_{j=1}^{N} \alpha_{j} \lambda_{j}\left(p_{k}\right)=0, \quad 1 \leq k \leq Q
$$

erfüllt.

\section{Zusatzbedingungen für die Kopplung in der Aeroelastik}

Wie in der Einleitung bereits beschrieben wurde, werden bei einer Kopplung in der Aeroelastik die von der Strömungssimulation berechneten Kräfte an die Struktursimulation übergeben. Die Struktursimulation berechnet die Verschiebungen aufgrund dieser Kräfte und gibt sie an die Strömungssimulation zurück. Dies lässt sich schematisch so darstellen:

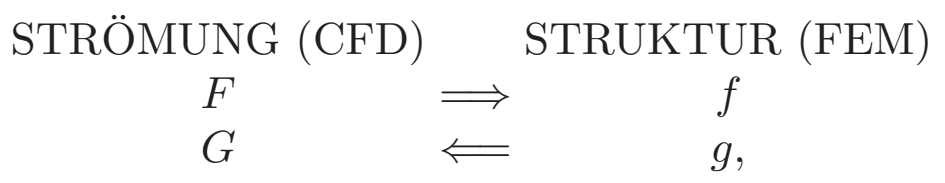

wobei von nun an folgende Bezeichnungen gelten sollen:

$-X=\left\{x_{1}, \ldots, x_{N}\right\}$ ist die Menge Strukturknoten,

$-Y=\left\{y_{1}, \ldots, y_{M}\right\}$ ist die Menge Strömungsknoten,

$-f=\left(f\left(x_{1}\right), \ldots, f\left(x_{N}\right)\right)$ sind die in der Kopplung zu berechnenden Kräfte auf der Strukturseite,

- $F=\left(F\left(y_{1}\right), \ldots, F\left(y_{M}\right)\right)$ sind die in der Kopplung durch den CFD-Solver gegebenen Kräfte auf der Strömungsseite,

$-g=\left(g\left(x_{1}\right), \ldots, g\left(x_{N}\right)\right)$ sind die in der Kopplung durch den FEM-Solver gegebenen Verschiebungen,

- $G=\left(g\left(y_{1}\right), \ldots, g\left(y_{M}\right)\right)$ sind die in der Kopplung zu berechnenden Verschiebungen auf der Strömungsseite.

Man beachte, dass die einzelnen Einträge $f\left(x_{j}\right), F\left(y_{j}\right), g\left(x_{j}\right), G\left(y_{j}\right)$ jeweils $d$-dimensionale Vektoren (mit $d=3$ ) sind. Daher wird von nun an z.B. mit $f_{j} \in \mathbb{R}^{N}, 1 \leq j \leq d$ die $j$-te Komponente der Matrix $f$ bezeichnet.

In der Regel werden die Interpolationsprobleme so angesetzt, dass die einzelnen Komponenten unabhängig voneinander modelliert werden. Eine Ausnahme findet in Abschnitt 2.4 statt. 
Bei einer Strömung-Struktur-Kopplung müssen verschiedene zusätzliche Bedingungen erfüllt werden, um bei der Simulation gewisse physikalische Größen im Gesamtsystem zu erhalten. Diese Bedingungen gehen auch in die räumliche Kopplung ein, wie im Folgenden gezeigt wird.

\subsection{Erhaltung der Arbeit}

Transformation der Werte mit der transponierten Kopplungsmatrix. Eine wichtige Bedingung für die Strömung-Struktur-Kopplung ist, dass die Arbeit im gesamten System erhalten bleibt. Es darf an der Kopplungsregion durch die Interpolation keine Arbeit gewonnen oder verloren werden. Dies führt zu einer zusätzlichen Bedingung an die Kopplung: Die Summe der Kraftkomponenten, multipliziert mit den entsprechenden Verschiebungskomponenten, muss auf Strömungs- und Strukturseite gleich sein. Die durch die Strömungskräfte geleistete Arbeit an der um die Deformationswerte verschobenen Strömungsoberfläche muss gleich der durch die Strukturkräfte um die Deformationswerte verschobenen Strukturoberfläche sein.

In der diskreten Situation der numerischen Berechnung ergibt sich folgende Bedingung:

$$
f_{j}^{T} g_{j}=F_{j}^{T} G_{j}, \quad 1 \leq j \leq d
$$

oder ausgeschrieben:

$$
\sum_{i=1}^{N} f_{j}\left(x_{i}\right) g_{j}\left(x_{i}\right)=\sum_{i=1}^{M} F_{j}\left(y_{i}\right) G_{j}\left(y_{i}\right), \quad 1 \leq j \leq d .
$$

Eine Möglichkeit, die Arbeitserhaltung zu gewährleisten, besteht darin, die für die Verschiebungen berechnete Kopplungsmatrix zu transponieren und die Transponierte für die Transformation der Kräfte zu verwenden. Die Kopplungsmatrix $H \in \mathbb{R}^{M \times N}$ ist diejenige Matrix, die $g_{j} \in \mathbb{R}^{N}$ auf $G_{j} \in \mathbb{R}^{M}$ für $1 \leq j \leq d$ abbildet. Sie lässt sich nach (2.7) als Produkt der Interpolationsmatrix mit einer Auswertungsmatrix schreiben. Dies ist allerdings hier nicht relevant.

Theorem 2.1 Sei $H \in \mathbb{R}^{M \times N}$ die für die Verschiebungen genutzte Kopplungsmatrix. d.h. es gelte $G_{j}=H g_{j}$ für $1 \leq j \leq d$. Wird $H^{T}$ zur Transformation der Kräfte benutzt, d.h. wird

$$
f_{j}=H^{T} F_{j}, \quad 1 \leq j \leq d
$$

gesetzt, dann gilt die diskrete Arbeitserhaltung

$$
\sum_{i=1}^{N} f_{j}\left(x_{i}\right) g_{j}\left(x_{i}\right)=\sum_{i=1}^{M} F_{j}\left(y_{i}\right) G_{j}\left(y_{i}\right), \quad 1 \leq j \leq d .
$$


Beweis: Die Voraussetzungen liefern

$$
f_{j}^{T} g_{j}=\left(H^{T} F_{j}\right)^{T} g_{j}=F_{j}^{T} H g_{j}=F_{j}^{T} G_{j}
$$

mit $1 \leq j \leq d$

Zusatzbedingung bei der Interpolation mit radialen Basisfunktionen. Die gerade beschriebene Transformation der Kräfte, die auf der Transponierten der Kopplungsmatrix für die Verschiebungen beruht, zeigt sehr unterschiedliche Resultate bei Verwendung verschiedener radialer Basisfunktionen. Dies führt zu sehr unterschiedlichen Ergebnissen bei Kopplungsläufen mit verschiedenen radialen Basisfunktionen, von denen einige keine brauchbaren Resultate in der gekoppelten Simulation ergeben. Mit der bisherigen Methode lässt sich dieses Phänomen aber nicht beseitigen, da die Matrix zur Interpolation der Verschiebungen bestimmt wird, und die Transponierte keine Interpolationseigenschaften besitzt. So können die verschiedenen radialen Basisfunktionen komplett unterschiedliche Kraftverteilungen über die transponierte Matrix produzieren.

Eine neue Idee ist, die Kräfte wie die Verschiebungen mit radialen Basisfunktionen zu interpolieren und entweder bei der Interpolation der Verschiebungen oder der Kräfte die Arbeitserhaltung als Zusatzbedingung zu fordern, um die Arbeit bei der gekoppelten Simulation zu erhalten.

Bei den Interpolationen über Zusatzbedingungen wird die zusätzliche Freiheit gewonnen, unterschiedliche Basisfunktionen für die Interpolation der Verschiebungen und die Interpolation der Kräfte zu benutzen.

Wie bereits erwähnt, können die Zusatzbedingungen sowohl bei der Interpolation der Verschiebungen als auch bei der Interpolation der Kräfte gefordert werden. Dementsprechend ist einmal ein klassisches und einmal ein verallgemeinertes Interpolationsproblem zu lösen. Im folgenden Satz wird die zusätzliche Bedingung bei der Interpolation der Kräfte eingebunden. Der Einfachheit halber werden Kräfte und Verschiebungen als univariate Größen betrachtet.

Theorem 2.2 Sei $\Phi$ bedingt positiv definit der Ordnung $m$. Die Menge $Y=$ $\left\{y_{1}, \ldots, y_{M}\right\} \subseteq \mathbb{R}^{d}$ sei $\Pi_{m-1}\left(\mathbb{R}^{d}\right)$ unisolvent und $X=\left\{x_{1}, \ldots, x_{N}\right\} \subseteq \mathbb{R}^{d}$ sei nicht in $Y$ enthalten. Ferner seien die Kräfte $F\left(y_{j}\right)$ und Verschiebungen $G\left(y_{j}\right), 1 \leq j \leq M$ auf Strömungsseite sowie die Verschiebungen $g\left(x_{j}\right), 1 \leq$ $j \leq N$ auf Strukturseite bekannt. Dann existiert genau eine Funktion der Form

$$
s(x)=\sum_{j=1}^{M} \alpha_{j} \Phi\left(x, y_{j}\right)+\alpha_{0} \sum_{j=1}^{N} g\left(x_{j}\right) \Phi\left(x, x_{j}\right)+\sum_{k=1}^{Q} \beta_{k} p_{k}(x),
$$

die die Interpolationsbedingungen $s\left(y_{j}\right)=F\left(y_{j}\right), 1 \leq j \leq M$ und

$$
\sum_{j=1}^{N} s\left(x_{j}\right) g\left(x_{j}\right)=\sum_{j=1}^{M} F\left(y_{j}\right) G\left(y_{j}\right)
$$


sowie die Nebenbedingung

$$
\sum_{j=1}^{M} \alpha_{j} p_{k}\left(y_{j}\right)+\alpha_{0} \sum_{j=1}^{N} p_{k}\left(x_{j}\right) g\left(x_{j}\right)=0, \quad 1 \leq k \leq Q
$$

erfüllt.

Beweis: Werden die $M+1$ Funktionale $\lambda_{j}, 0 \leq j \leq M \operatorname{durch} \lambda_{j}(\gamma)=\gamma\left(y_{j}\right)$ für $1 \leq j \leq M$ und $\lambda_{0}(\gamma)=\sum_{j=1}^{N} g\left(x_{j}\right) \gamma\left(x_{j}\right)$ definiert, so kann die Funktion $s$ aus $(2.10)$ als

$$
s(x)=\sum_{j=0}^{M} \alpha_{j} \lambda_{j}^{y} \Phi(x, y)+\sum_{k=1}^{Q} \beta_{k} p_{k}(x)
$$

geschrieben werden und die $M+1$ Interpolationsbedingungen lauten nun $\lambda_{j}(s)=F\left(y_{j}\right)$ für $1 \leq j \leq M$ und $\lambda_{0}(s)=\sum_{j=1}^{M} F\left(y_{j}\right) G\left(y_{j}\right)$. Nach Theorem 1.8 reicht es also nachzuweisen, dass die $\lambda_{j}$ linear unabhängig sind.

Ist nun $x_{\ell} \in X$ einer der Punkte, die nicht in $Y$ liegen, so können in

$$
\sum_{j=0}^{M} \alpha_{j} \lambda_{j}(\gamma)=0
$$

nacheinander Funktionen $\gamma$ eingesetzt werden, die jeweils auf einem Punkt aus $\left\{x_{\ell}\right\} \cup Y$ eins sind und auf allen anderen Punkten aus $X \cup Y$ null. Dann verschwindet der zugehörige Koeffizient $\alpha_{j}$, was die lineare Unabhängigkeit zeigt.

Die neuen Kräfte auf der Strukturseite sind dann natürlich durch Auswerten von $s$ auf $X$ gegeben. Um ein konkretes Interpolationsproblem zu lösen, muss wie oben diskutiert, die Matrix $A_{\Phi, \Lambda}$ zur Bestimmung der Koeffizienten $\alpha=\left(\alpha_{0}, \alpha_{1}, \ldots, \alpha_{M}\right) \in \mathbb{R}^{M+1}$ und $\beta \in \mathbb{R}^{Q}$ invertiert werden. Diese Matrix hat jetzt die konkrete Form

$$
A_{\Phi, \Lambda}=\left(\begin{array}{ccc}
a & b^{T} & c^{T} \\
b & A & P \\
c & P^{T} & 0
\end{array}\right)
$$

wobei $A=A_{\Phi, Y}=\left(\Phi\left(y_{i}, y_{j}\right)\right) \in \mathbb{R}^{M \times M}$ und $P=P_{Y}=\left(p_{j}\left(y_{i}\right)\right) \in \mathbb{R}^{M \times Q}$ die schon zuvor aufgetretenen Matrizen sind. Die übrigen Einträge ergeben sich $\mathrm{zu}$

$$
\begin{aligned}
& a=\lambda_{0}^{x} \lambda_{0}^{y} \Phi(x, y)=\sum_{j, k=1}^{N} g\left(x_{j}\right) g\left(x_{k}\right) \Phi\left(x_{j}, x_{k}\right), \\
& b_{j}=\lambda_{0}^{x} \lambda_{j}^{y} \Phi(x, y)=\sum_{k=1}^{N} g\left(x_{k}\right) \Phi\left(y_{j}, x_{k}\right), \quad 1 \leq j \leq M, \\
& c_{j}=\lambda_{0}\left(p_{j}\right)=\sum_{k=1}^{N} g\left(x_{k}\right) p_{j}\left(x_{k}\right), \quad 1 \leq j \leq Q .
\end{aligned}
$$




\subsection{Konservativität}

Transformation der Kräfte mit der transponierten Kopplungsmatrix. Eine weitere Größe, die bei Kopplungen in der Aeroelastik zu erhalten ist, ist die Summe der Kräfte. Man spricht in diesem Zusammenhang auch von Konservativität. Wie im Abschnitt 2.1 gezeigt wurde, besteht eine Möglichkeit, die Arbeit in der Kopplung zu erhalten, darin, die Transponierte der berechneten Kopplungsmatrix $H$ der Verschiebungen aus (2.7) zur Transformation der Kräfte zu benutzen. Diese Transponierte, der auf Grundlage von radialen Basisfunktionen unter Hinzunahme eines konstanten polynomialen Terms aufgestellten Kopplungsmatrix, transformiert die Werte konservativ, wie im Folgenden bewiesen wird. Dies bedeutet, dass, um die Summe der Kräfte zu erhalten, alle Basisfunktionen als bedingt positiv definite Funktionen von mindestens der Ordnung $m=1$ aufgefasst werden müssen. Insbesondere sind auch positiv definite Funktionen mindestens mit konstanten Polynomen zu rechnen.

Theorem 2.3 Sei $H$ die für die Verschiebungen genutzte Kopplungsmatrix aus (2.7), d.h. es gelte $G_{j}=H g_{j}$ für $1 \leq j \leq d$. Treten dort mindestens konstante Polynome in $P$ auf und wird $H^{T}$ zur Transformation der Kräfte benutzt, d.h. wird

$$
f_{j}=H^{T} F_{j}, \quad 1 \leq j \leq d
$$

gesetzt, dann gilt die diskrete Erhaltung der Kraft

$$
\sum_{i=1}^{N} f_{j}\left(x_{i}\right)=\sum_{i=1}^{M} F_{j}\left(y_{i}\right), \quad 1 \leq j \leq d .
$$

Beweis: Mit den zuvor eingeführten Bezeichnungen gilt mit (2.7) zunächst

$$
\begin{aligned}
\left(\begin{array}{c}
f_{j} \\
\tilde{f}_{j}
\end{array}\right) & =\left(\begin{array}{c}
H^{T} \\
\widetilde{H}^{T}
\end{array}\right) F_{j}=\left[\left(\begin{array}{ll}
A_{\Phi, X, Y} & P_{Y}
\end{array}\right)\left(\begin{array}{cc}
A_{\Phi, X} & P_{X} \\
P_{X}^{T} & 0
\end{array}\right)^{-1}\right]^{T} F_{j} \\
& =\left(\begin{array}{cc}
A_{\Phi, X} & P_{X} \\
P_{X}^{T} & 0
\end{array}\right)-\left(\begin{array}{c}
A_{\Phi, X}^{T}, Y \\
P_{Y}^{T}
\end{array}\right) F_{j}
\end{aligned}
$$

jeweils für $1 \leq j \leq d$ mit einem Vektor $\tilde{f}_{j} \in \mathbb{R}^{Q}$. Hieraus folgt zunächst

$$
\left(\begin{array}{cc}
A_{\Phi, X} & P_{X} \\
P_{X}^{T} & 0
\end{array}\right)\left(\begin{array}{c}
f_{j} \\
\widetilde{f}_{j}
\end{array}\right)=\left(\begin{array}{c}
A_{\Phi, X, Y}^{T} F_{j} \\
P_{Y}^{T} F_{j}
\end{array}\right)
$$

und damit durch Vergleich des unteren Blockes auch

$$
P_{X}^{T} f_{j}=P_{Y}^{T} F_{j}, \quad 1 \leq j \leq d,
$$

was ausgeschrieben gerade

$$
\sum_{i=1}^{N} p\left(x_{i}\right) f_{j}\left(x_{i}\right)=\sum_{i=1}^{M} p\left(y_{i}\right) F_{j}\left(y_{i}\right), \quad 1 \leq j \leq d
$$


für beliebiges $p \in \Pi_{m-1}\left(\mathbb{R}^{d}\right)$ bedeutet. Damit liefert die Wahl $p(x)=1$ die Behauptung.

Man beachte, dass bei dieser Wahl der Transformation der Kräfte nicht nur die diskrete Arbeit erhalten wird. Vielmehr wurde gerade gezeigt, dass auch diskrete Momente von höherer Ordnung erhalten bleiben. Schließlich gilt (2.12) für jedes Polynom $p \in \Pi_{m-1}\left(\mathbb{R}^{d}\right)$.

Zusatzbedingung in der Interpolation mit radialen Basisfunktionen. Wie im Abschnitt 2.1 bereits beschrieben wurde, zeigt die Transformation der Kräfte über die Transponierte verschiedene Probleme.

Eine zweite Möglichkeit, die Kräfte unter der Bedingung der Konservativität zu transformieren, ist, ähnlich wie bei der Erhaltung der Arbeit, die Kräfte wie die Verschiebungen zu interpolieren und die Konservativität als Zusatzbedingung zu fordern. Die Strömungspunkte sind dann die Stützstellen und an den Strukturpunkten erfolgt die Auswertung.

Der Einfachheit halber werden Kräfte und Verschiebungen wieder als univariate Größen aufgefasst. Die resultierenden Interpolationsmatrizen können für alle drei Koordinatenrichtungen gleichermaßen benutzt werden.

Theorem 2.4 Sei $\Phi$ bedingt positiv definit der Ordnung $m$. Die Menge $Y=$ $\left\{y_{1}, \ldots, y_{M}\right\} \subseteq \mathbb{R}^{d}$ sei $\Pi_{m-1}\left(\mathbb{R}^{d}\right)$ unisolvent und $\left\{x_{1}, \ldots, x_{N}\right\} \subseteq \mathbb{R}^{d}$ sei nicht in $Y$ enthalten. Gegeben seien ferner die Kräfte $F\left(y_{1}\right), \ldots, F\left(y_{M}\right)$ auf Strömungsseite. Dann existiert genau eine Funktion der Form

$$
s(x)=\sum_{j=1}^{M} \alpha_{j} \Phi\left(x, y_{j}\right)+\alpha_{0} \sum_{j=1}^{N} \Phi\left(x, x_{j}\right)+\sum_{k=1}^{Q} \beta_{k} p_{k}(x),
$$

die die Interpolationsbedingungen $s\left(y_{j}\right)=F\left(y_{j}\right), 1 \leq j \leq M$ und

$$
\sum_{j=1}^{N} s\left(x_{j}\right)=\sum_{j=1}^{M} F\left(y_{j}\right)
$$

sowie die Nebenbedingungen

$$
\sum_{j=1}^{M} \alpha_{j} p_{k}\left(y_{j}\right)+\alpha_{0} \sum_{j=1}^{N} p_{k}\left(x_{j}\right)=0, \quad 1 \leq k \leq Q
$$

erfüllt.

Beweis: Wie im Beweis zu Theorem 2.2 wird der Satz unter Benutzung von Funktionalen formuliert. Mit $\lambda_{j}(\gamma)=\gamma\left(y_{j}\right), 1 \leq j \leq M$ und $\lambda_{0}(\gamma)=$ $\sum_{j=1}^{N} \gamma\left(x_{j}\right)$ lässt sich die gesuchte Funktion schreiben als

$$
s(x)=\sum_{j=0}^{M} \alpha_{j} \lambda_{j}^{y} \Phi(x, y)+\sum_{k=1}^{Q} \beta_{k} p_{k}(x) .
$$


Die Interpolationsbedingungen sind $\lambda_{j}(s)=F\left(y_{j}\right)$ für $1 \leq j \leq M$ und $\lambda_{0}(s)=\sum_{j=1}^{M} F\left(y_{j}\right)$. Die Nebenbedingung lautet $\sum_{j=0}^{M} \alpha_{j} \lambda_{j}(p)=0$ für alle $p \in \Pi_{m-1}\left(\mathbb{R}^{d}\right)$. Also reicht es nach Theorem 1.8 wieder nachzuweisen, dass die $\lambda_{j}$ linear unabhängig sind. Dies geschieht hier genauso wie im Beweis zu Theorem 2.2.

Um ein konkretes Interpolationsproblem zu lösen, muss die Matrix zur Bestimmung der Koeffizienten konkret aufgestellt werden. Sie hat hier wieder die Form

$$
A_{\Phi, \Lambda}=\left(\begin{array}{ccc}
a & b^{T} & c^{T} \\
b & A & P \\
c & P^{T} & 0
\end{array}\right)
$$

mit $A=A_{\Phi, Y}=\left(\Phi\left(y_{i}, y_{j}\right)\right) \in \mathbb{R}^{M \times M}$ und $P=P_{Y}=\left(p_{j}\left(y_{i}\right)\right) \in \mathbb{R}^{M \times Q}$. Die übrigen Einträge ergeben sich diesmal zu

$$
\begin{aligned}
& a=\lambda_{0}^{x} \lambda_{0}^{y} \Phi(x, y)=\sum_{j, k=1}^{N} \Phi\left(x_{j}, x_{k}\right), \\
& b_{j}=\lambda_{0}^{x} \lambda_{j}^{y} \Phi(x, y)=\sum_{k=1}^{N} \Phi\left(y_{j}, x_{k}\right), \quad 1 \leq j \leq M, \\
& c_{j}=\lambda_{0}\left(p_{j}\right)=\sum_{k=1}^{N} p_{j}\left(x_{k}\right), \quad 1 \leq j \leq Q .
\end{aligned}
$$

Die Kräfte zu interpolieren steht in einem gewissen Widerspruch zu der physikalischen Bedeutung der den Elementen zugeordneten Kräfte. Diese Interpolation kann aber als Verteilung unter der Bedingung der Konservativität angesehen werden. Sie wird insbesondere in Verbindung mit den in Abschnitt $3 \mathrm{zu}$ besprechenden, lokalen Verfahren interessant, da dann die Verteilung lokal beschränkt und damit zu einem gewissen Grad auch noch physikalisch sinnvoll bleibt.

\subsection{Erhaltung der Arbeit und Konservativität}

Transformation der Kräfte mit der transponierten Kopplungsmatrix. Für die transponierte Matrix ist der Beweis zur gleichzeitigen Konservativität und Erhaltung der Arbeit bereits durch die beiden Beweise für Konservativität und Arbeitserhaltung gegeben.

Zusatzbedingung in der Interpolation mit radialen Basisfunktionen. Durch Aufstellung der Bedingungen zu Konservativität und Arbeitserhaltung in einem Gleichungssystem, so wie sie zuvor einzeln gefordert worden sind, werden beide Bedingungen erhalten. Die Interpolante wird im Ansatz um zwei statt nur um ein zusätzliches Auswertungsfunktional erweitert. Die Nebenbedingungen sind entsprechend zu modifizieren. 
Wenn für die Transformation der Kräfte bei einer Anwendung mit sehr unterschiedlichen Anzahlen von Strömungs- und Strukturknoten die Interpolation mit Zusatzbedingungen zu Konservativität und Arbeitserhaltung verwendet wird, so muss Folgendes bedacht werden: Sei z.B. die Anzahl der Strömungsknoten wesentlich höher als die der Strukturknoten und der Wert für eine Kraftkomponente sei auf allen Strömungsknoten größer null. Die folgenden Erklärungen gelten natürlich genau umgekehrt auch für eine höhere Anzahl von Strukturknoten als Strömungsknoten, was in wirklichen Anwendungen aber nicht vorkommt. Die Annahme, dass alle Werte größer null sind, ist nur auf Grund der besseren Veranschaulichung getroffen worden, das Problem sowie der Lösungsansatz gelten genauso ohne diese Annahme.

Da die Summe der Kraftkomponenten auf Grund der Konservativitätsbedingung aufsummiert auf allen Strömungs- und Strukturknoten gleich sein muss, müssen die Strukturknoten, da es weniger sind, wesentlich höhere Werte erhalten. Liegen nun einige der Strukturknoten auf den Strömungsknoten, so werden diese Strukturknoten durch die Interpolationsbedingung den Wert des jeweiligen Strömungsknotens erhalten. Vereinfacht gesagt, würde eine solche Konstellation in einer Anwendung auf den Strukturknoten, die nicht auf Strömungsknoten liegen, grundsätzlich höhere Werte ergeben als auf den Strukturknoten, die auf Strömungsknoten liegen. Dies würde wieder zu sehr unregelmäßigen Ergebnissen führen und der qualitative Verlauf der Werte auf den Strömungsknoten würde so nicht wiedergegeben.

Da die Kräfte als Ergebnis des Integrals des Drucks über die Fläche eines Strömungselements gesehen werden können, ist eine Interpolation der Kraft im eigentlichen Sinne, nicht das, was wirklich gefordert ist. Gefordert ist vielmehr die Verteilung der Kräfte in qualitativ gleicher Weise wie auf der Strömungsseite unter der Bedingung der Konservativität und der Erhaltung der Arbeit in der gekoppelten Simulation. Dass die Kraftkomponente an einem Strukturknoten, der auf einem Strömungsknoten liegt, gleich der Kraftkomponente auf dem Strömungsknoten ist, wie es normalerweise in der Interpolation gefordert wird, muss bei der Krafttransformation nicht Bedingung sein. Über die Annahme, dass bei einer größeren Anzahl von Strömungsknoten als Strukturknoten die Flächen der Strömungselemente kleiner sind als die Flächen der Strukturelemente auf dem Kopplungsgebiet, kann der regelmäßige Verlauf der Werte der Strömungsknoten für die interpolierten Werte auf den Strukturknoten erzielt werden. Dies kann z.B. dadurch geschehen, dass die Elementgrößen in die jeweiligen Funktionale mit eingebaut werden. Am einfachsten lässt sich aber ein Faktor, der Quotient aus der Anzahl der Strömungsknoten und der Anzahl der Strukturknoten ist, berechnen und mit den Werten der Kraftkomponenten auf den Strömungsknoten multiplizieren. Die resultierenden Werte stellen dann die Werte, die in die Interpolationsbedingung eingehen. So werden, wie in den Anwendungen zu sehen sein wird (siehe Kapitel IV Abschnitt 5.1), auch bei solchen Konstellationen für die Kopplung gute Ergebnisse erreicht. Physikalisch ist dies sinnvoll, solange die 
in Beziehung gesetzten Knoten nahe beieinander liegen, wie es bei den noch zu besprechenden lokalen Verfahren der Fall ist.

\subsection{Rotationen}

Die Interpolationen sollten möglichst alle gegebenen Informationen in der Strömung-Struktur-Kopplung verarbeiten können. Der Strukturcode liefert in einigen Anwendungen außer den Verschiebungen auf den Knoten auch Rotationswerte. In den bisherigen Codes und wissenschaftlichen Untersuchungen wurden in den Interpolationen auf Grundlage radialer Basisfunktionen die berechneten Rotationen nicht für die Interpolation verwendet. Gerade aber in den Kopplungen z.B. mit Mehrkörpersimulationscodes sind die zur Verfügung stehenden Daten sehr gering. So ist es sehr wichtig, die Rotationswerte an den Knoten in der Interpolation verwenden zu können. Ein solches Verfahren wird im Folgenden für den dreidimensionalen Raum entwickelt.

Für kleine Winkel können die Rotationen über die Beziehung

$$
\left(\begin{array}{c}
\theta_{u} \\
\theta_{v} \\
\theta_{w}
\end{array}\right)=\frac{1}{2}\left(\begin{array}{ccc}
0 & -\frac{\partial}{\partial w} & \frac{\partial}{\partial v} \\
\frac{\partial}{\partial w} & 0 & -\frac{\partial}{\partial u} \\
-\frac{\partial}{\partial v} & \frac{\partial}{\partial u} & 0
\end{array}\right)\left(\begin{array}{c}
u \\
v \\
w
\end{array}\right)=: \frac{1}{2} \nabla \times\left(\begin{array}{c}
u \\
v \\
w
\end{array}\right)
$$

als Ableitungen der Verschiebungen in die Interpolationen einbezogen werden (siehe auch [19]). Wenn die Rotationen in dieser Weise in die Interpolationen eingehen, tritt das zusätzliche Problem auf, dass die Koordinatenrichtungen nicht mehr unabhängig voneinander modelliert werden können. Vielmehr muss mit echten, vektorwertigen Interpolanten gearbeitet werden. In der Theorie der radialen Basisfunktionen wird dabei zu matrixwertigen Basisfunktionen übergegangen, die es erlauben, sogar zusätzliche Nebenbedingungen in die Interpolante einzubauen (siehe [20]).

Im Folgenden seien die Strukturknoten $X$ die Stützstellen und die Strömungsknoten $Y$ die Auswertungspunkte.

An den $N$ Stützstellen, $x_{1}, \ldots, x_{N} \in \mathbb{R}^{3}$, seien für die drei Koordinatenrichtungen die Verschiebungswerte

$$
g\left(x_{i}\right)=\left(g_{1}\left(x_{i}\right), g_{2}\left(x_{i}\right), g_{3}\left(x_{i}\right)\right)^{T} \in \mathbb{R}^{3}, \quad 1 \leq i \leq N,
$$

und die Rotationswerte

$$
\theta\left(x_{i}\right)=\left(\theta_{1}\left(x_{i}\right), \theta_{2}\left(x_{i}\right), \theta_{3}\left(x_{i}\right)\right)^{T} \in \mathbb{R}^{3}, \quad 1 \leq i \leq N
$$

gegeben. Gesucht wird nun eine Interpolante $s: \mathbb{R}^{3} \rightarrow \mathbb{R}^{3}$, die die Bedingungen

$$
\begin{aligned}
s\left(x_{i}\right) & =g\left(x_{i}\right), & 1 \leq i \leq N \\
(\nabla \times s)\left(x_{i}\right) & =2 \theta\left(x_{i}\right), & 1 \leq i \leq N
\end{aligned}
$$


erfüllt. Die einzelnen Komponenten dieser vektorwertigen Interpolanten können nun nicht mehr unabhängig von einander modelliert werden.

Entscheidend ist hierbei auch, dass es sich eigentlich um $6 N$ Interpolationsbedingungen handelt, so dass auch mindestens $6 \mathrm{~N}$ Unbekannte in den Interpolationsansatz eingebaut werden müssen. Um den richtigen Ansatz zu finden, ist es daher sinnvoll die Interpolationsbedingungen wieder mit Funktionalen zu schreiben. Diesmal geht es allerdings um vektorwertige Funktionale $\lambda=\left(\lambda_{1}, \lambda_{2}, \lambda_{3}\right)$, und die Aktion von $\lambda$ auf eine vektorwertige Funktion $s=\left(s_{1}, s_{2}, s_{3}\right): \mathbb{R}^{3} \rightarrow \mathbb{R}^{3}$ wird definiert durch

$$
\lambda(s):=\lambda_{1}\left(s_{1}\right)+\lambda_{2}\left(s_{2}\right)+\lambda_{3}\left(s_{3}\right),
$$

siehe [20]. Dies bedeutet, dass zunächst die Punktauswertungsfunktionale

$$
\lambda_{3 j-2}:=\left(\begin{array}{c}
\delta_{x_{j}} \\
0 \\
0
\end{array}\right), \lambda_{3 j-1}:=\left(\begin{array}{c}
0 \\
\delta_{x_{j}} \\
0
\end{array}\right), \lambda_{3 j}:=\left(\begin{array}{c}
0 \\
0 \\
\delta_{x_{j}}
\end{array}\right),
$$

für $1 \leq j \leq N$ gegeben sind. Hierbei bezeichnet $\delta_{x}$ das Punktauswertungsfunktional an der Stelle $x$, also $\delta_{x}(f)=f(x)$. Es muss beachtet werden, dass auf Grund von (2.13) diese Funktionale tatsächlich das gewünschte liefern. Es gilt z.B. $\lambda_{3 j-2}(s)=s_{1}\left(x_{j}\right)+0+0=s_{1}\left(x_{j}\right)$. Als nächstes sind die Funktionale für die Rotationen für $1 \leq j \leq N$ gegeben durch

$$
\begin{aligned}
\lambda_{3(N+j)-2} & =\left(\begin{array}{c}
0 \\
-\delta_{x_{j}} \circ \frac{\partial}{\partial w} \\
\delta_{x_{j}} \circ \frac{\partial}{\partial v}
\end{array}\right) \\
\lambda_{3(N+j)-1} & =\left(\begin{array}{c}
\delta_{x_{j}} \circ \frac{\partial}{\partial w} \\
0 \\
-\delta_{x_{j}} \circ \frac{\partial}{\partial u}
\end{array}\right) \\
\lambda_{3(N+j)} & =\left(\begin{array}{c}
-\delta_{x_{j}} \circ \frac{\partial}{\partial v} \\
\delta_{x_{j}} \circ \frac{\partial}{\partial u} \\
0
\end{array}\right),
\end{aligned}
$$

wobei für die Ableitungen die Koordinatenrichtungen mit $u, v, w$ bezeichnet wurden. Diese Funktionale erfüllen wieder die gewünschte Darstellung. Es gilt z.B.

$$
\lambda_{3(N+j)-2}(s)=0-\frac{\partial s_{2}}{\partial w}\left(x_{j}\right)+\frac{\partial s_{3}}{\partial v}\left(x_{j}\right),
$$

was der ersten Komponente von $(\nabla \times s)\left(x_{j}\right)$ entspricht. Diese Funktionale erlauben nun den Ansatz

$$
s(x)=\sum_{j=1}^{6 N} \alpha_{j} \lambda_{j}^{y}\left(\begin{array}{ccc}
\Phi_{1}(x, y) & 0 & 0 \\
0 & \Phi_{2}(x, y) & 0 \\
0 & 0 & \Phi_{3}(x, y)
\end{array}\right)+p(x)
$$


mit einem vektorwertigen Polynom $p$ und drei (möglicherweise unterschiedlichen) Basisfunktionen $\Phi_{i}(x, y)$. Die Anwendung des Funktionals auf eine Matrix erfolgt zeilenweise. Komponentenweise ausgeschrieben bedeutet dies

$$
\begin{aligned}
& s_{1}=\sum_{j=1}^{N}\left[\alpha_{3 j-2} \Phi_{1}\left(\cdot, x_{j}\right)+\alpha_{3(N+j)-1} \frac{\partial_{2} \Phi_{1}\left(\cdot, x_{j}\right)}{\partial w}-\alpha_{3(N+j)} \frac{\partial_{2} \Phi_{1}\left(\cdot, x_{j}\right)}{\partial v}\right]+p_{1} \\
& s_{2}=\sum_{j=1}^{N}\left[\alpha_{3 j-1} \Phi_{2}\left(\cdot, x_{j}\right)-\alpha_{3(N+j)-2} \frac{\partial_{2} \Phi_{2}\left(\cdot, x_{j}\right)}{\partial w}+\alpha_{3(N+j)} \frac{\partial_{2} \Phi_{2}\left(\cdot, x_{j}\right)}{\partial u}\right]+p_{2} \\
& s_{3}=\sum_{j=1}^{N}\left[\alpha_{3 j} \Phi_{3}\left(\cdot, x_{j}\right)+\alpha_{3(N+j)-2} \frac{\partial_{2} \Phi_{3}\left(\cdot, x_{j}\right)}{\partial v}-\alpha_{3(N+j)-1} \frac{\partial_{2} \Phi_{3}\left(\cdot, x_{j}\right)}{\partial u}\right]+p_{3},
\end{aligned}
$$

wobei der zusätzliche Index 2 an $\partial_{2}$ hier die Ableitung von $\Phi$ bzgl. des zweiten Arguments bedeutet. In der Regel wird $\Phi_{1}=\Phi_{2}=\Phi_{3}=$ : $\Phi$ gewählt werden, sofern die konkrete Anwendung nicht etwas anderes nahe legt.

Ist $\Phi$ bedingt positiv definit der Ordnung $m$, so sind $p_{1}, p_{2}, p_{3} \in \Pi_{m-1}\left(\mathbb{R}^{3}\right)$ zu wählen, und zu den $6 N$ Interpolationsbedingungen

$$
\begin{array}{lll}
\lambda_{3 j-2}(s)=g_{1}\left(x_{j}\right), & \lambda_{3 j-1}(s)=g_{2}\left(x_{j}\right), & \lambda_{3 j}(s)=g_{3}\left(x_{j}\right) \\
\lambda_{3(N+j)-2}(s)=2 \theta_{1}\left(x_{j}\right), & \lambda_{3(N+j)-1}(s)=2 \theta_{2}\left(x_{j}\right), & \lambda_{3(N+j)}(s)=2 \theta_{3}\left(x_{j}\right)
\end{array}
$$

für $1 \leq j \leq N$ muss noch zusätzlich

$$
\sum_{j=1}^{6 N} \alpha_{j} \lambda_{j}(q)=0, \quad q=\left(q_{1}, q_{2}, q_{3}\right), q_{i} \in \Pi_{m-1}\left(\mathbb{R}^{d}\right)
$$

gefordert werden.

Um ein konkretes Interpolationsproblem zu lösen, muss wiederum die Matrix zur Bestimmung des Koeffizientenvektors $\alpha \in \mathbb{R}^{6 N}$ und des Koeffizientenvektors von $p$ aufgestellt werden. Diese Matrix ist wieder nach Konstruktion symmetrisch und hat wieder einen Blockcharakter, der durch die verschiedenen Funktionaltypen bestimmt ist. Eine explizite Angabe wird hier aus offensichtlichen Gründen unterdrückt. Sie lässt sich mit Hilfe des Ansatzes sowie der Interpolations- und Zusatzbedingungen aufstellen.

Wichtig ist hier nur die Wohldefiniertheit des Ansatzes, also die Invertierbarkeit der Interpolationsmatrix. Es gilt der folgende Satz:

Theorem 2.5 Die oben definierten Funktionale $\lambda_{j}, 1 \leq j \leq N$ sind linear unabhängig. Daher ist die zugehörige Interpolationsmatrix invertierbar.

Beweis: Wenn angenommen wird, dass $\sum_{j=1}^{6 N} \alpha_{j} \lambda_{j}(s)=0$ für alle hinreichend glatten, vektorwertigen Funktionen $s$ gilt, dann kann für jedes $1 \leq j \leq$ $N$ eine glatte Funktion $g: \mathbb{R}^{3} \rightarrow \mathbb{R}$ gewählt werden, die einen kompakten 
Träger hat, der nur den Datenpunkt $x_{k}$ enthält. Ferner soll $g\left(x_{k}\right)=1$ und $\nabla g\left(x_{k}\right)=0$ gelten. Wird nun $s=(g, 0,0)$ gesetzt, so wird

$$
\begin{aligned}
0 & =\sum \alpha_{j} \lambda_{j}(s) \\
& =\alpha_{3 k-2} g\left(x_{k}\right)+\alpha_{3(N+k)-1} \frac{\partial g\left(x_{k}\right)}{\partial w}-\alpha_{3(N+k)} \frac{\partial g\left(x_{k}\right)}{\partial v} \\
& =\alpha_{3 k-2}
\end{aligned}
$$

erhalten. Für die übrigen Koeffizienten muss dieser Ansatz entsprechend modifiziert werden.

\section{Das „Partition of Unity“6 Verfahren}

\subsection{Allgemeine Überlegungen}

Die radialen Basisfunktionen mit globalem Träger haben den Nachteil, dass sie hinsichtlich der Rechenzeit sehr teuer sind. Direkte Methoden, um die Koeffizienten für die Interpolante zu bestimmen, benötigen bei $N$ Stützstellen $\mathcal{O}\left(N^{3}\right)$ Operationen. Bei der Auswertung der Interpolante kommen noch $\mathcal{O}(N)$ Operationen für jede Auswertungsstelle hinzu. Bei Problemen mit mehr als 10000 Punkten ist dies mit akzeptablem Aufwand nicht mehr zu bewältigen.

Mit den in der Ausgangsbibliothek implementierten Interpolationen ist es z.B. nicht möglich, große Gesamtkonfiguration eines Flugzeugs zu bearbeiten. Es können nur Teile von Strömungs- und Struktur-Kopplungsflächen in die Algorithmen eingehen. Dies führt zu Unstetigkeitsstellen an den Übergängen der Teilgebiete. Mit den folgenden Verfahren werden Rechenzeit- und Speicherplatzanforderungen derart verbessert, dass auch große Gesamtkonfigurationen in eine räumliche Kopplung eingehen können.

Die im Folgenden präsentierte Idee ist von Wendland in [10] vorgestellt worden. Die Methode löst viele kleine, lokale Interpolationsprobleme mit radialen Basisfunktionen und setzt die lokalen Ergebnisse dann mit einem „Partition of Unity" (PoU) Verfahren zu einer Gesamtlösung zusammen.

Dabei sieht die prinzipielle Vorgehensweise folgendermaßen aus. Zuerst wird das interessierende Gebiet $\Omega$, welches die Stützstellen und Auswertungsstellen enthält, mit kleineren Gebieten $\left\{\Omega_{j}\right\}_{j=1}^{n}$ überdeckt:

$$
\Omega \subseteq \bigcup_{j=1}^{n} \Omega_{j}
$$

Die Anforderungen an die Überdeckung werden im Weiteren näher spezifiziert. Zu dieser Überdeckung wird eine Familie von stetigen, nichtnegativen 
Funktionen $\left\{w_{j}\right\}$ mit kompaktem Träger gewählt, mit den Eigenschaften

$$
\sum_{j=1}^{n} w_{j}(x)=1, \quad x \in \Omega
$$

und $\operatorname{supp}\left(w_{j}\right) \subseteq \overline{\Omega_{j}}$. Schließlich wird noch zu jeder Zelle $\Omega_{j}$ ein Approximationsraum $V_{j}$ gewählt.

Um nun die globale Zielfunktion $f \in C(\Omega)$ zu approximieren, wird sie zunächst lokal auf $\Omega_{j}$ durch ein $s_{j} \in V_{j}$ approximiert. Die lokalen Approximanten werden dann über die folgende Gleichung zu einer globalen Approximanten auf dem Ausgangsgebiet $\Omega$ zusammengefügt:

$$
s_{f}(x)=\sum_{j=1}^{n} s_{j}(x) w_{j}(x) .
$$

Die so allgemein definierte globale Approximante erbt die Eigenschaften der lokalen Approximanten:

Theorem 3.1 Der globale Fehler zwischen der Zielfunktion $f \in C(\Omega)$ und $s_{f}$ lässt sich beschränken durch das Maximum der lokalen Fehler:

$$
\left\|f-s_{f}\right\|_{L_{\infty}(\Omega)} \leq \max _{1 \leq j \leq n}\left\|f-s_{j}\right\|_{L_{\infty}\left(\Omega_{j}\right)}
$$

Beweis: Wegen (2.17) gilt $f(x)=\sum f(x) w_{j}(x)$, so dass wegen des kompakten Trägers und der Nichtnegativität der $w_{j}$ sofort

$$
|f(x)-s(x)| \leq \sum_{j=1}^{n}\left|f(x)-s_{j}(x)\right| w_{j}(x) \leq \max _{1 \leq j \leq n}\left\|f-s_{j}\right\|_{L_{\infty}\left(\Omega_{j}\right)} \sum_{j=1}^{n} w_{j}(x)
$$

gefolgert werden kann, was mit (2.17) die Behauptung liefert.

Dies bedeutet, dass eine gute lokale Approximation an $f$ auch zu einer guten globalen Approximation von $f$ führt.

Wichtig in diesem Zusammenhang ist noch die Eigenschaft, dass zusätzliche Eigenschaften der lokalen Funktionen oft von der globalen Funktion geerbt werden. Sind z.B. die lokalen Funktionen in ihrem Gebiet Interpolanten, so ist die globale Funktion ebenfalls eine Interpolante.

Theorem 3.2 Sei $X=\left\{x_{1}, \ldots, x_{N}\right\} \subseteq \Omega$ gegeben. Wenn $X$ gemäß der Zerlegung (2.16) in Teilmengen $X_{j}=X \cap \Omega_{j}$ zerlegt wird und die lokalen Approximanten als Interpolanten auf $X_{j}$ gewählt werden, so interpoliert $s_{f}$ die unbekannte Funktion in ganz $X$.

Beweis: Wenn für $x \in \Omega$ mit $I(x)=\left\{j: x \in \Omega_{j}\right\}$ die Indizes bezeichnet werden, für die höchstens $w_{j}(x)>0$ gilt, so folgt

$$
s_{f}\left(x_{i}\right)=\sum_{j=1}^{n} s_{j}\left(x_{i}\right) w_{j}\left(x_{i}\right)=\sum_{j \in I\left(x_{i}\right)} f\left(x_{i}\right) w_{j}\left(x_{i}\right)=f\left(x_{i}\right),
$$


für $1 \leq i \leq N$.

Bei den Zusatzbedingungen wie Erhaltung der Arbeit, Konservativität, Rotationen, muss die lokale verallgemeinerte Interpolationsaufgabe modifiziert werden, damit die globale Approximante die verallgemeinerte Aufgabe erfüllt. Dies wird später noch genauer erläutert.

Zuvor soll hier auf die Wahl der Zellen $\Omega_{j}$ eingegangen werden. Für diese Wahl müssen folgende Dinge beachtet werden:

1. Jede Zelle soll eine im Vergleich zu $N$ kleine und von $N$ unabhängige Anzahl $N_{j}=\mathcal{O}(1)$ Punkte enthalten. Dies ist wichtig, da dann jede der $n$ Interpolationsaufgaben in $\mathcal{O}\left(N_{j}^{3}\right)=\mathcal{O}(1)$ Zeit gelöst werden kann. Andererseits bedeutet dies, dass $n=\mathcal{O}(N)$ Probleme zu lösen sind. Der Gesamtaufwand für die Lösung summiert sich daher zunächst zu $\mathcal{O}(N)$.

2. Jeder Punkt $x \in \Omega$ soll höchstens in $\mathcal{O}(1)$ vielen Zellen liegen. Anders ausgedrückt, soll die Menge $I(x)=\left\{j: x \in \Omega_{j}\right\}$ eine als konstant anzusehende Kardinalität haben. Daraus folgt dann, dass, wenn $I(x)$ bekannt ist, die globale Interpolante auch in konstanter Zeit ausgewertet werden kann.

3. Werden die lokalen Interpolanten mit radialen Basisfunktionen gebildet, so müssen die lokalen Gebiete $\Omega_{j}$ noch gewisse, gleichmäßige Regularitätsbedingungen erfüllen. Dies ist z.B. bei Quader-förmigen Gebieten der Fall.

Details zum letzten Punkt können in [4] nachgelesen werden.

Die Fehlerabschätzungen bei solchen scattered-data Interpolationsproblemen haben immer die Form

$$
\left\|f-s_{f}\right\|_{L_{\infty}(\Omega)} \leq C h_{X, \Omega}^{\tau}\|f\|_{\Phi}
$$

Dabei wird angenommen, dass $f$ aus einem der Basisfunktion $\Phi$ zugeordneten und mit $\|\cdot\|_{\Phi}$ normierten Raum kommt. Die Ordnung $\tau>0$ hängt ebenfalls von $\Phi$ ab. Gemessen wird der Fehler in Ermangelung eines Gitters in Abhängigkeit von der sogenannten Fülldichte

$$
h_{X, \Omega}=\sup _{x \in \Omega} \min _{x_{j} \in X}\left\|x-x_{j}\right\|_{2} .
$$

Sie gibt den Radius der größten, noch ganz in $\Omega$ enthaltenen Kugel an, die keinen Datenpunkt enthält und ist daher ein Maß dafür, wie gut $X$ das Gebiet $\Omega$ "ausfüllt". Eine ausführliche Darstellung dieses Sachverhalts ist wieder in [4] zu finden. Dort kann auch nachgelesen werden, dass $s_{f}$ nicht nur $f$, sondern die Ableitungen von $s_{f}$ auch die Ableitungen von $f$ gut approximieren. Dazu muss allerdings noch eine zusätzliche Bedingung an die Gewichte $w_{j}$ gefordert werden, nämlich, dass der Trägerradius mit $h_{X, \Omega}$ skaliert, dies ist bei annähernd gleich verteilten Punkten leicht zu erreichen.

Bei der Anwendung in der Aeroelastik sind solche Approximationssätze allerdings nur von beschränktem Nutzen, da die Stützstellen in der Regel auf 
Oberflächen liegen, so dass die Fülldichte auf die „Bounding Box“ bezogen relativ groß ist. Ferner erfolgen oft Auswertungen außerhalb der „Bounding Box" der Stützstellen, so dass es sich eigentlich um Extrapolationsverfahren handelt. Dennoch lassen sich mit diesen Verfahren sehr gute Ergebnisse erzielen.

Für eine effiziente Implementierung des Partition of Unity Verfahrens, ist es, in Bezug auf die obigen Punkte 1. und 2., erforderlich, sicher zu stellen, dass

- die Punkte $X$ effizient den Mengen $X_{j}=X \cap \Omega_{j}$ zugeordnet werden können,

- für jedes $x$ die Indexmenge $I(x)$ effizient bestimmt werden kann.

Dies soll nun näher betrachtet werden.

\subsection{Algorithmen zur Aufteilung des Gebietes in Zellen.}

Nachdem im ersten Abschnitt gezeigt worden ist, dass sich bei Verwendung radialer Basisfunktionen, kombiniert mit der PoU Methode, die Konvergenzordnung im Vergleich zu den Interpolationen, die direkt mit radialen Basisfunktionen arbeiten, nicht verschlechtert, wird in diesem Teil ein konkreter Algorithmus vorgestellt. Im nächsten Abschnitt werden zu diesem Algorithmus noch Aufwandsabschätzungen zur Rechenzeit gegeben, um die Effizienzsteigerung durch diese Methode zu belegen.

Das zentrale Problem in der Konstruktion eines effizienten Algorithmus für die PoU Methode, kombiniert mit radialen Basisfunktionen, ist, eine geeignete Überdeckung $\left\{\Omega_{j}\right\}_{j=1}^{n}$ des Interpolationsgebietes $\Omega$ zu finden, welche den im Abschnitt 3.1 definierten Anforderungen genügt. Für die Effizienz ist es entscheidend, dass der Algorithmus eine schnelle Konstruktion der Gebiete auf Grundlage der Stützstellen einerseits und eine schnelle Bestimmung der jeweils enthaltenen Auswertungsstellen andererseits zulässt. So muss schnell entschieden werden können, in welchem $\Omega_{j}$ ein gegebener Punkt $x \in \Omega$ liegt. Hier existieren bereits einige effiziente Verfahren, wie z.B. „kd-trees“ oder „bd-trees“, die in [9] beschrieben werden. Diese Verfahren müssten allerdings noch für die speziellen Anforderungen in der PoU Methode, kombiniert mit radialen Basisfunktionen, angepasst werden.

In dieser Arbeit wird ein neuer Algorithmus vorgestellt, der für diese Anforderungen implementiert worden ist und für die Berechnungen mit der PoU Methode in den in Kapitel IV präsentierten Anwendungen verwendet wird. Der Algorithmus wird jetzt für ein Interpolationsgebiet im zweidimensionalen Raum vereinfacht beschrieben. Das Verfahren wird unter der Annahme erläutert, dass alle auftretenden Divisionen ganzzahlige Werte liefern. Dies geschieht, um das Prinzip zu verdeutlichen und durch die einzelnen Schwierigkeiten der Implementation dieses nicht zu überdecken. So werden hier alle Details des Verfahrens ausgeblendet. Das Verfahren ist natürlich für $d$ dimensionale Räume und beliebige Anzahlen von Stützstellen implementiert worden. 
Seien $N$ Stützstellen $X=\left\{x_{1}, \cdots, x_{N}\right\} \subseteq \mathbb{R}^{2}$ gegeben. Die Koordinatenrichtungen seien mit $u$ und $v$ bezeichnet. Es sollen Zellen $\Omega_{j}$ konstruiert werden, in denen ungefähr $p \cdot o^{2}$ Punkte vorhanden sind. Die Bedeutung von $p$ und $o$ ergibt sich im Folgenden. Der Exponent von $o$ ist die Raumdimension und $o$ selbst ist der Überlappbereich zu anderen Zellen in der gleichen Raumrichtung, wobei der Algorithmus für jede Raumrichtung einmal durchgeführt wird. Konkreter heißt dies, dass o Zellen in jeder Raumrichtung einen Überlapp mit einer Zelle haben, ausgenommen die Zellen $\Omega_{j}$, die an den Rändern des zu überdeckenden Interpolationsgebietes $\Omega$ liegen. Die Zahl $p$ ist die Anzahl der Punkte in einer Zelle, wenn der Überlapp gleich eins ist.

Der Algorithmus beginnt mit der Sortierung der $N$ Stützstellen nach ihrer Koordinate in Richtung $u$ in aufsteigender Reihenfolge. Dann werden nach jeweils $\frac{N}{\sqrt{\frac{N}{p}}}=\sqrt{N \cdot p}$ Punkten Trennungen eingezogen, so dass sich $\sqrt{\frac{N}{p}}$ Mengen von Stützstellen ergeben. Von diesen Mengen werden immer $o$ durch die Sortierung aufeinanderfolgende Mengen zu einer neuen Menge zusammengenommen, so dass die erste zusammengesetzte Menge aus der ersten Menge bis zur o-ten Menge, die zweite aus der zweiten Menge bis zur $(o+1)$-ten Menge usw. besteht. Der jeweils erste Punkt dieser Menge und der Punkt, der auf den letzten Punkt der Menge in der Sortierung folgt, geben dann die Grenzen der sich aus dieser Menge ergebenden Zellen in die $u$-Koordinatenrichtung an. Im nächsten Schritt werden dann die Stützstellen der einzelnen aus dem letzten Schritt resultierenden Mengen nach ihrer $v$ Koordinate wiederum in aufsteigender Reihenfolge sortiert. Dann werden in jeder dieser Mengen wieder Trennschnitte nach $\frac{o \cdot \sqrt{N \cdot p}}{\sqrt{\frac{N}{p}}}=p \cdot o$ Punkten eingezogen, so dass sich jeweils eine Anzahl von $\sqrt{\frac{N}{p}}$ Mengen ergibt. Hier werden wieder $o$ in der aktuellen Sortierung aufeinanderfolgende Mengen - in gleicher Weise wie vorher - zusammengesetzt. Die zusammengesetzten Mengen dürfen allerdings nur Mengen aus einer Ursprungsmenge sein, es darf beispielsweise nicht die letzte Menge der ersten Ursprungsmenge mit der ersten Menge der zweiten Ursprungsmenge vereint werden. Auch hier werden wieder der erste und der in der Sortierung auf den letzten einer Menge folgende Punkt zur Bestimmung der Grenze, der zu bildenden Zellen in v-Koordinatenrichtung, benutzt. Damit wird folgende Anzahl von gleichmäßig überlappenden Zellen konstruiert:

$$
n=\left(\sqrt{\frac{N}{p}}-(o-1)\right)^{2}
$$

wobei die Zellen $p \cdot o^{2}$ Punkte enthalten. In einem $d$-dimensionalen Raum resultieren aus dem Algorithmus

$$
n=\left(\sqrt[d]{\frac{N}{p}}-(o-d+1)\right)^{d}
$$


gleichmäßig überlappende Zellen jeweils mit einer Anzahl von $p \cdot o^{d}$ Punkten.

Da natürlich die Stützstellen-Anzahl im Allgemeinen nicht so gegeben ist, dass sich bei den Divisionen immer ganzzahlige Ergebnisse erzielen lassen, werden die sich ergebenden Reste bei den Divisionen möglichst gleichmäßig auf die Zellen verteilt. Der Algorithmus ist so implementiert, dass gewährleistet ist, dass auch bei Restbildungen in den Divisionen die Anzahl der Stützstellen in einer der am Ende des Algorithmus resultierenden überlappenden Zellen immer kleiner als $p \cdot\left(o^{d}+1\right)$ ist.

Der Algorithmus wird dann erneut gestartet, wobei nun mit der Sortierung der Stützstellen nach ihrer $v$-Koordinate begonnen und dann ebenso weiter verfahren wird wie vorher. Bei der zweiten Sortierung wird dann nach der $u$-Koordinate sortiert. In einer Anwendung mit mehr als zwei Raumdimensionen werden die Sortierungskoordinaten bei jedem neuen Start des Algorithmus zyklisch vertauscht.

Nach Beendigung dieses Algorithmus müssen die an den Rändern liegenden Zellen evtl. vergrößert werden, wenn das Interpolationsgebiet $\Omega$ größer sein soll als die Vereinigung der sich ergebenden Zellen.

In den Abbildungen 1 und 2 werden die Algorithmen für beide Sortierungen an Hand der sich ergebenden Partitionen für zwei Raumdimensionen verdeutlicht. Das Beispiel hat 75 Stützstellen und einen Überlapp von $o=2$ mit $p=3$. So werden Zellen mit jeweils $p \cdot o^{2}=12$ Stützstellen konstruiert.

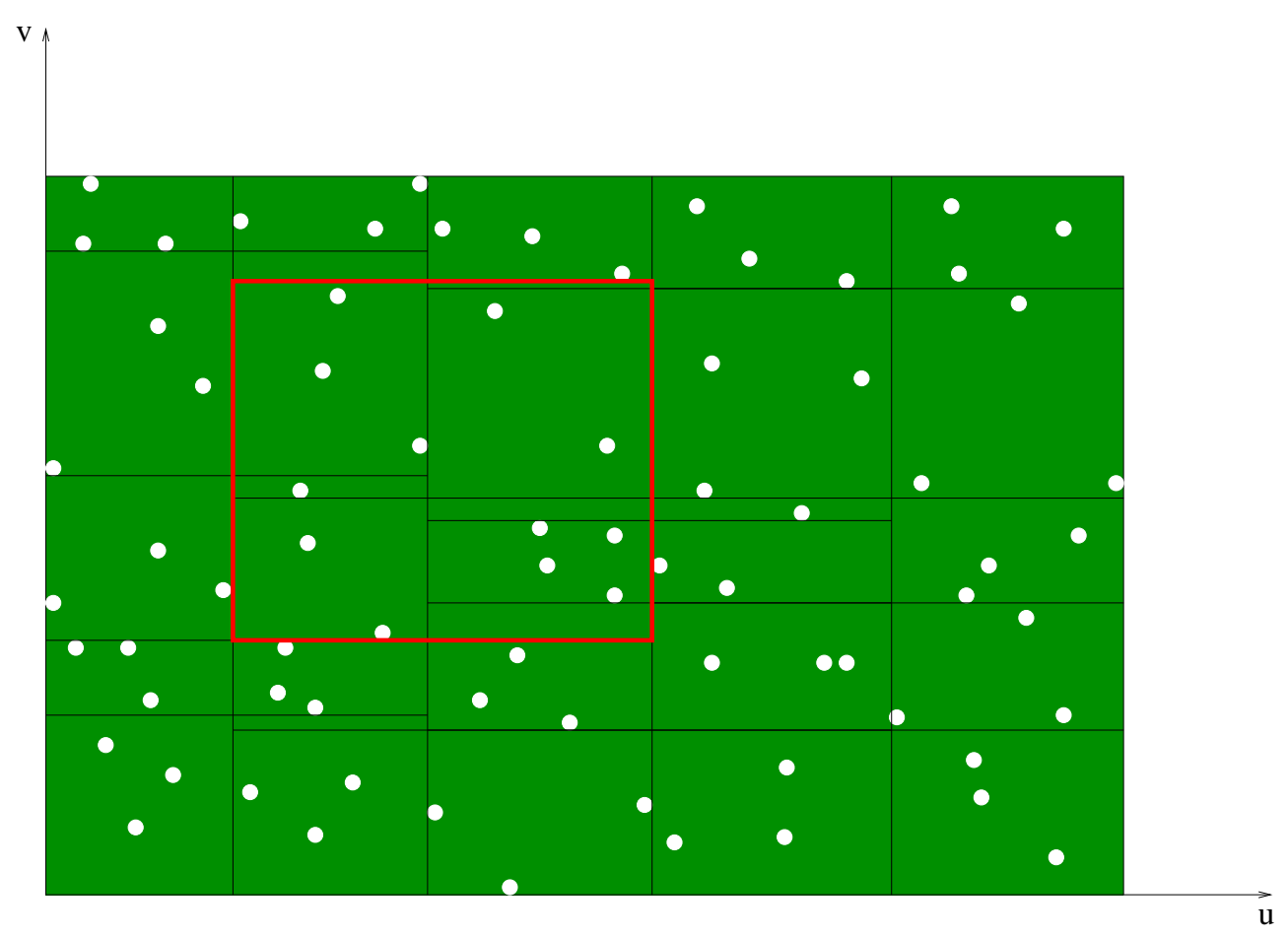

Abbildung 1. Die Skizze zeigt die Konstruktion der Zellen, wobei zuerst nach der $u$-Koordinate und dann nach der $v$-Koordinate sortiert wird. Das rote Rechteck zeigt beispielhaft eine Zelle. 


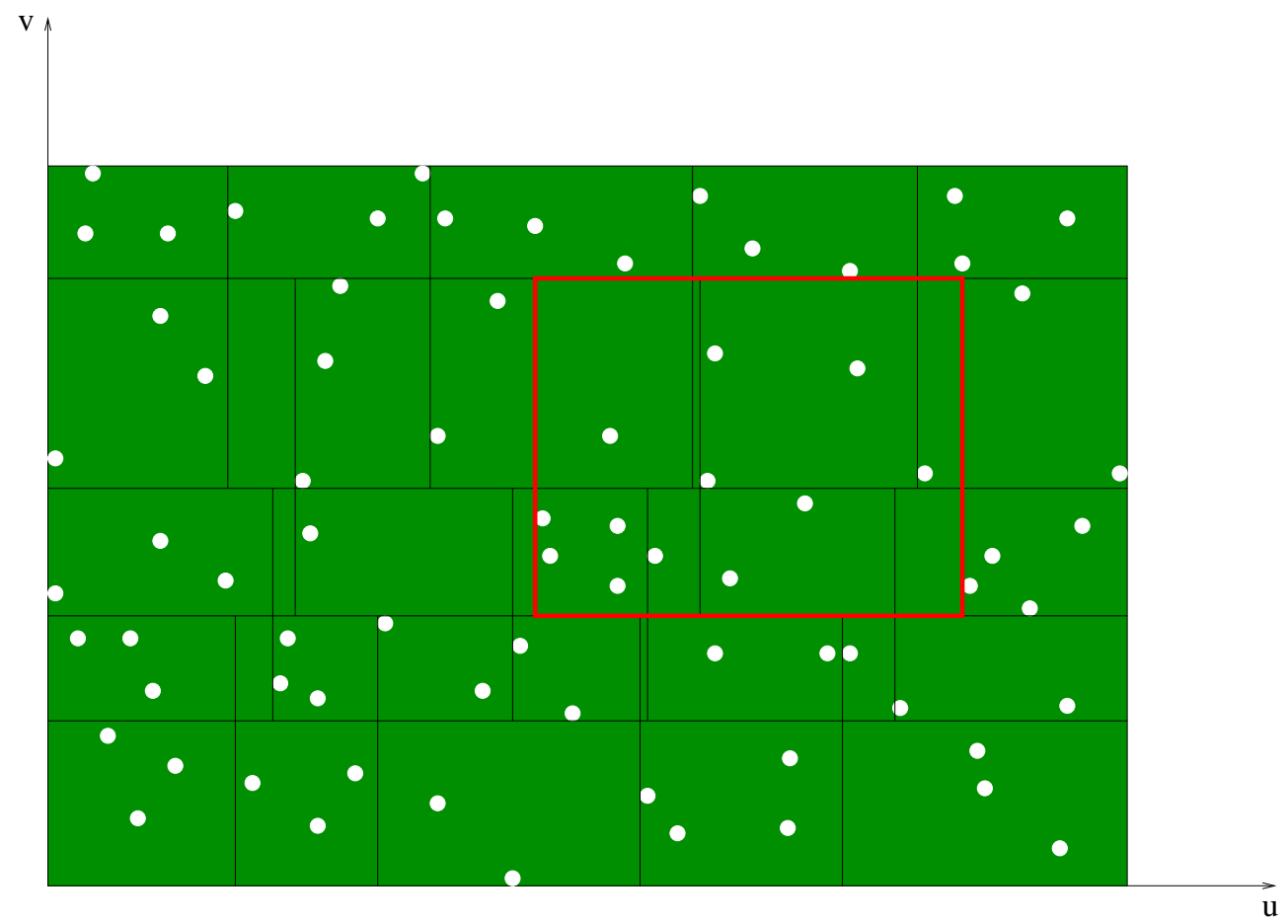

Abbildung 2. Die Skizze zeigt die Konstruktion der Zellen, wobei zuerst nach der $v$-Koordinate und dann nach der $u$-Koordinate sortiert wird. Das rote Rechteck zeigt beispielhaft eine Zelle.

Der Algorithmus wird im Folgenden ACITA-Algorithmus genannt. In den Algorithmus kann auf verschiedenste Art und Weise über die Änderung von Default-Parameterwerten eingegriffen werden. Als erstes können die Koordinatenrichtungen, in die geteilt werden soll, bestimmt werden. Es kann also z.B. bei einem dreidimensionalen Interpolationsgebiet auch nur in zwei Richtungen geteilt werden. In den Algorithmus gehen dann nur zwei Koordinaten zur Konstruktion der Zellen ein. Außerdem können die Koordinatenrichtungen in unterschiedlich viele Mengen aufgeteilt werden, wenn z.B. die Ausdehnung in die verschiedenen Koordinatenrichtungen stark unterschiedlich ist. Bei großen auftretenden Löchern zwischen den Stützstellen kann in einem bestimmten Rahmen eingestellt werden, wie weit die Zellen in diesem Bereich vergrößert werden sollen.

\subsection{Komplexität des Algorithmus}

Im Folgenden wird die Komplexität des ACITA-Algorithmus zur Konstruktion der Zellen und der darauf aufbauenden PoU Methode kombiniert mit radialen Basisfunktionen bestimmt. Der Rechenzeitaufwand für die Konstruktion der Zellen ist abhängig von der Effizienz des zugrundeliegenden Sortieralgorithmus. In der hier zugrundeliegenden Implementation werden die Sortieralgorithmen der C++ Standard Template Library Klasse „Map“ (siehe [21]) verwendet. Die Komplexität dieser Algorithmen lässt sich laut der 
Literatur bei $N$ Stützstellen mit

$$
\mathcal{O}\left(\sum_{i=1}^{N} \log i\right) \leq \mathcal{O}(N \log N)
$$

abschätzen, was für einen Sortieralgorithmus optimal ist. Da in dem Konstruktionsalgorithmus die Zuordnung der Stützstellen zu den Zellen inbegriffen ist, muss bei diesem Algorithmus für die Zuordnung kein zusätzlicher Aufwand addiert werden.

Theorem 3.3 Für $N$ beliebig verteilte Stützstellen ist der Aufwand zur Konstruktion der Zellen mit Zuordnung der Stützstellen zu den Zellen unter Verwendung des ACITA-Algorithmus $\mathcal{O}(N \log N)$.

Die Anzahl $n$ der Zellen ist, wie oben beschrieben, proportional zu der Anzahl $N$ der Stützstellen. Da jede Zelle nach Konstruktion höchstens $p$. $\left(o^{d}+1\right)$ Stützstellen enthält, kann jedes einzelne, lokale Gleichungssystem in $\mathcal{O}\left(\left(p \cdot\left(o^{d}+1\right)\right)^{3}\right)=\mathcal{O}(1)$ Zeit gelöst werden. Da es $\mathcal{O}(N)$ viele sind, kostet das Lösen also insgesamt $\mathcal{O}(N)$ Zeit.

Was die Auswertung angeht, liegt jede Auswertungsstelle nach Konstruktion ebenfalls nur in konstant vielen Zellen. Sind diese bekannt, kostet die einzelne Auswertung also $\mathcal{O}(1)$ Zeit. Um die Zellen, also $I(x)$, zu ermitteln, wird zunächst für jeden "Durchgang" mit binärer Suche für jede einzelne Koordinate diejenige Zelle bestimmt, die $x$ enthält. Dies führt zu $\mathcal{O}\left(\log ^{d}(N)\right)$ Operationen. Ausgehend von dieser Zelle muss nur o Zellen "gegen" die Suchrichtung zurückgegangen werden, da $x$ nur in diesen zusätzlichen Zellen liegen kann. Dieser zusätzliche Schritt kann in konstanter Zeit ausgeführt werden. Insgesamt ergibt sich die Komplexität des ACITA-Algorithmus zu:

Theorem 3.4 Sei $X=\left\{x_{1}, \cdots, x_{N}\right\} \subseteq \Omega$ eine Menge ungleichmäßig verteilter Stützstellen. Die Überdeckungen $\left\{\Omega_{j}\right\}$ können mit einem Rechenzeitaufwand von $\mathcal{O}(N \log N)$ berechnet werden. Die anschließende Berechnung der lokalen Interpolanten kostet $\mathcal{O}(N)$ Zeit und jede einzelne Auswertung kann in $\mathcal{O}\left(\log ^{d}(N)\right)$ Zeit erfolgen.

Der Aufwand von $\left(\log ^{d}(N)\right)$ für eine einzelne Auswertung ist ein wenig zu hoch. Er lässt sich für den Fall von $M$ simultanen Auswertungen leicht verbessern.

\subsection{Zusatzbedingungen}

Um auch Zusatzbedingungen wie die Erhaltung der Arbeit und Konservativität in das $\mathrm{PoU}$ Verfahren einzubinden, gibt es prinzipiell wieder zwei Möglichkeiten: Entweder wird die transponierte der Kopplungsmatrix genutzt, oder die Zusatzbedingungen werden wieder in die Interpolationsbedingungen eingebunden. Letzteres ist auch die einzige Möglichkeit, die Rekonstruktion von Rotationen mit PoU zu kombinieren. 
Allerdings muss dann der Ansatz leicht variiert werden. Dies lässt sich am Einfachsten wieder im Fall von Funktionalen beschreiben: Sollen die gegebenen Werte $\lambda_{1}(f), \ldots, \lambda_{N}(f)$ durch eine Funktion $s_{f}$ der Form (2.18) rekonstruiert werden, so kann aus

$$
\lambda_{i}\left(s_{f}\right)=\sum_{j=1}^{n} \lambda_{i}\left(s_{i} w_{i}\right) \stackrel{!}{=} \lambda_{i}(f), \quad 1 \leq i \leq N
$$

gesehen werden, dass die Gewichte $w_{j}$ in die Funktionale zu den lokalen Problemen mit eingebaut werden müssen.

\subsection{Erhaltung der Arbeit}

Transformation der Werte mit der transponierten Kopplungsmatrix. Zur Erhaltung der Arbeit in einem Interpolationsverfahren mit der „Partition of Unity" Methode kann auch die Transponierte zur Transformation der Kräfte verwendet werden. „Partition of Unity“ Methode steht hier und im Folgenden für die „Partition of Unity“ Methode kombiniert mit radialen Basisfunktionen, wie sie im Abschnitt 3.1 eingeführt wurde.

Da in Theorem 2.1 keine weiteren Voraussetzungen über die Struktur der Kopplungsmatrix gemacht wurden, kann es direkt auch auf die Kopplungsmatrix aus dem PoU Verfahren angewendet werden und erhält also auch hier die Erhaltung der Arbeit.

Zusatzbedingung in der Interpolation mit radialen Basisfunktionen. Bei der zweiten Möglichkeit zur Erhaltung der Arbeit in der Kopplung, müssen die Zusatzbedingungen in einem PoU Verfahren, wie bereits oben erwähnt, für die Partitionen leicht abgeändert werden, um global die Arbeitserhaltung zu realisieren. Die Summen der Arbeitskomponenten multipliziert mit den entsprechenden Wichtungsfaktoren an den Stützstellen der Partition müssen in den lokalen Gleichungssystemen gleichgesetzt werden mit den Summen der Arbeitskomponenten multipliziert mit den entsprechenden Wichtungsfaktoren an den Auswertungsstellen der Partition. Die Bedingung wird im Folgenden kurz erläutert. Zunächst werden die notwendigen Notationen wiederholt bzw. eingeführt. Es bezeichne

- $\left\{\Omega_{k}\right\}_{k=1}^{n}$ die Zerlegung von $\Omega$ mit zugehöriger Teilung der Eins $\left\{w_{j}\right\}_{k=1}^{n}$,

- $X^{(k)}=\left\{x_{1}^{(k)}, \ldots, x_{N^{(k)}}^{(k)}\right\} \subseteq \mathbb{R}^{d}$ und $Y^{(k)}=\left\{y_{1}^{(k)}, \ldots, y_{M^{(k)}}^{(k)}\right\} \subseteq \mathbb{R}^{d}$ jeweils die Menge der Struktur- bzw. Strömungsknoten in der Zelle $\Omega_{k}$,

- $f\left(x_{j}^{(k)}\right)$ und $g\left(x_{j}^{(k)}\right)$ für $1 \leq j \leq N^{(k)}$ die Kräfte bzw. Verschiebungen auf der Strukturseite für Knoten in Zelle $\Omega_{k}$,

- $F\left(y_{j}^{(k)}\right)$ und $G\left(y_{j}^{(k)}\right)$ für $1 \leq j \leq M^{(k)}$ die Kräfte bzw. Verschiebungen auf der Strömungsseite für Knoten in Zelle $\Omega_{k}$. 
Wichtig ist hier noch, dass für Punkte $x$ in der offenen Menge $\Omega_{k}$ stets $w_{k}(x)>0$ gilt.

Wird die Zusatzbedingung wie in Theorem 2.2 beim Übergang von der Strömung zur Struktur gefordert, so sind die Größen $F\left(y_{j}^{(k)}\right), G\left(y_{j}^{(k)}\right)$ und $g\left(x_{j}^{(k)}\right)$ gegeben, und $f\left(x_{j}^{(k)}\right)$ ist als Wert der lokalen Interpolanten $s_{k}$ an $x_{j}^{(k)}$ zu berechnen. Die lokale Interpolante $s_{k}$ muss neben den Interpolationsbedingungen $s_{k}\left(y_{j}^{(k)}\right)=F\left(y_{j}^{(k)}\right), 1 \leq j \leq M^{(k)}$ noch die zusätzliche Bedingung

$$
\sum_{j=1}^{N^{(k)}} w_{k}\left(x_{j}^{(k)}\right) s_{k}\left(x_{j}^{(k)}\right) g\left(x_{j}^{(k)}\right)=\sum_{j=1}^{M^{(k)}} w_{k}\left(y_{j}^{(k)}\right) F\left(y_{j}^{(k)}\right) G\left(y_{j}^{(k)}\right)
$$

erfüllen, die sich von der globalen Arbeitserhaltungsbedingung (2.11) durch die zusätzlichen Gewichte unterscheidet. Ist diese Bedingung für alle $1 \leq k \leq$ $n$ erfüllt, so folgt für die globale Interpolante

$$
s=\sum_{k=1}^{n} s_{k} w_{k}
$$

die globale Erhaltung aus:

$$
\begin{aligned}
\sum_{j=1}^{N} s\left(x_{j}\right) g\left(x_{j}\right) & =\sum_{j=1}^{N} \sum_{k=1}^{n} s_{k}\left(x_{j}\right) w_{k}\left(x_{j}\right) g\left(x_{j}\right) \\
& =\sum_{k=1}^{n} \sum_{j=1}^{N^{(k)}} s_{k}\left(x_{j}^{(k)}\right) w_{k}\left(x_{j}^{(k)}\right) g\left(x_{j}^{(k)}\right) \\
& =\sum_{k=1}^{n} \sum_{j=1}^{M^{(k)}} w_{k}\left(y_{j}^{(k)}\right) F\left(y_{j}^{(k)}\right) G\left(y_{j}^{(k)}\right) \\
& =\sum_{j=1}^{M} \sum_{k=1}^{n} w_{k}\left(y_{j}\right) F\left(y_{j}\right) G\left(y_{j}\right)=\sum_{j=1}^{M} F\left(y_{j}\right) G\left(y_{j}\right) .
\end{aligned}
$$

Um die lokalen Interpolanten wieder symmetrisch anzusetzen, werden die Funktionale $\lambda_{j}^{(k)}(\gamma):=\gamma\left(y_{j}^{(k)}\right), 1 \leq j \leq M^{(k)}$ und

$$
\lambda_{0}^{(k)}(\gamma):=\sum_{j=1}^{N^{(k)}} w_{k}\left(x_{j}^{(k)}\right) g\left(x_{j}^{(k)}\right) \gamma\left(x_{j}^{(k)}\right)
$$

benutzt. Damit bekommt die lokale Interpolante komponentenweise die Form

$$
\begin{aligned}
s_{k} & =\sum_{j=0}^{M^{(k)}} \alpha_{j} \lambda^{y} \Phi(\cdot, y)+p^{(k)} \\
& =\alpha_{0} \sum_{j=1}^{N^{(k)}} w_{k}\left(x_{j}^{(k)}\right) g\left(x_{j}^{(k)}\right) \Phi\left(\cdot, x_{j}^{(k)}\right)+\sum_{j=1}^{M^{(k)}} \alpha_{j} \Phi\left(\cdot, y_{j}^{(k)}\right)+p^{(k)},
\end{aligned}
$$


wobei $p^{(k)}$ wieder ein Polynom aus $\Pi_{m-1}\left(\mathbb{R}^{d}\right)$ ist. Die Koeffizienten sind wieder wie im globalen Fall durch die Interpolationsbedingungen

$$
\begin{aligned}
& s_{k}\left(y_{j}^{(k)}\right)=F\left(y_{j}^{(k)}\right), \quad 1 \leq j \leq M^{(k)} \\
& \lambda_{0}\left(s_{k}\right)=\sum_{j=1}^{M} w_{k}\left(y_{j}^{(k)}\right) F\left(y_{j}^{(k)}\right) G\left(y_{j}^{(k)}\right)
\end{aligned}
$$

sowie die Nebenbedingungen

$$
\sum_{j=0}^{M^{(k)}} \lambda_{j}^{(k)}(q)=0, \quad q \in \Pi_{m-1}\left(\mathbb{R}^{d}\right)
$$

bestimmt. Die eindeutige Existenz von $s_{k}$ folgt wegen der Positivität der $w_{k}$ auf $\Omega_{k}$ aus Theorem 2.2. Dies ergibt zusammen den folgenden Satz.

Theorem 3.5 Sei $\Phi$ bedingt positiv definit. Es gelte mit obigen Bezeichnungen, dass für $1 \leq k \leq n$ die Menge $Y^{(k)}$ unisolvent bzgl. $\Pi_{m-1}\left(\mathbb{R}^{d}\right)$ ist und dass $X^{(k)}$ nicht in $Y^{(k)}$ enthalten ist. Dann gibt es für $1 \leq k \leq n$ genau eine Funktion $s_{k}$ der Form (2.20), die die Bedingungen (2.21) und (2.22) erfüllt. Die globale Funktion $s:=\sum s_{k} w_{k}$ erfüllt dann die verallgemeinerten Interpolationsbedingungen $s\left(y_{j}\right)=F\left(y_{j}\right), 1 \leq j \leq M$ und

$$
\sum_{j=1}^{N} s\left(x_{j}\right) g\left(x_{j}\right)=\sum_{j=1}^{M} F\left(y_{j}\right) G\left(y_{j}\right) .
$$

Man beachte, dass neben dieser globalen Erhaltung der Arbeit auch eine gewichtete, lokale Erhaltung bei dieser Vorgehensweise garantiert wird. Es gilt schließlich für jede Zelle $\Omega_{k}$ die zusätzliche Bedingung (2.19). Dies ist physikalisch wesentlich realistischer als nur die globale Erhaltung der Arbeit über die zusätzliche Interpolation zu fordern.

\subsection{Konservativität}

Transformation der Kräfte mit der transponierten Kopplungsmatrix. Wenn bei einer Interpolation mit der „Partition of Unity“ Methode Konservativität gefordert wird, so kann, wie bei der globalen Anwendung von radialen Basisfunktionen, die Transponierte der über die „Partition of Unity“ Methode berechneten dünn besetzten Matrix verwendet werden, sofern mindestens ein konstanter polynomialer Term bei der Berechnung der lokalen Interpolante hinzugenommen wird.

Theorem 3.6 Sei $H$ die für die Verschiebungen genutzte Kopplungsmatrix aus dem PoU Verfahren, d.h. es gelte $G_{j}=H g_{j}$ für $1 \leq j \leq d$. Werden 
die lokalen Interpolanten mindestens mit konstanten Polynomen gebildet und wird $H^{T}$ zur Transformation der Kräfte benutzt, d.h. wird

$$
f_{j}=H^{T} F_{j}, \quad 1 \leq j \leq d
$$

gesetzt, dann gilt die diskrete Erhaltung der Kraft

$$
\sum_{i=1}^{N} f_{j}\left(x_{i}\right)=\sum_{i=1}^{M} F_{j}\left(y_{i}\right), \quad 1 \leq j \leq d .
$$

Beweis: Die Kopplungsmatrix kann explizit aufgestellt werden und dann ähnlich wie im Beweis zu Theorem 2.3 argumentiert werden. Es geht aber auch einfacher. Da die lokalen Interpolanten mindestens konstante Polynome exakt reproduzieren, gilt dies auch für die globale Interpolante. Dies bedeutet aber für die Kopplungsmatrix $H \in \mathbb{R}^{M \times N}$, dass sie angewandt auf den Vektor $e_{N}:=(1, \ldots, 1)^{T} \in \mathbb{R}^{N}$ den Vektor $e_{M}=(1, \ldots, 1)^{T} \in \mathbb{R}^{M}$ liefert:

$$
H e_{N}=e_{M}
$$

Dies bedeutet aber sofort

$$
\sum_{i=1}^{N} f_{j}\left(x_{i}\right)=f_{j}^{T} e_{N}=F_{j}^{T} H e_{N}=F_{j}^{T} e_{M}=\sum_{i=1}^{M} F_{j}\left(y_{i}\right)
$$

für $1 \leq j \leq d$

Der Beweis zeigt, dass jeder Interpolationsprozess, der Konstanten exakt reproduziert, über die Transponierte Kopplungsmatrix zur Konservativität führt. Insbesondere ließe sich auch Theorem 2.3 so beweisen.

Ferner lässt sich dieses Argument auf Polynome höheren Grades erweitern.

Zusatzbedingung in der Interpolation mit radialen Basisfunktionen. Wie in Abschnitt 2.2 beschrieben, kann die Konservativität auch durch eine Zusatzbedingung in der Interpolation realisiert werden, welches die schon erläuterten Vorteile für die Kopplung bringt. Wird diese Zusatzbedingung mit der „Partition of Unity" Methode eingesetzt, müssen die lokalen Matrizen wiederum so angepasst werden, dass global die Konservativität gewährleistet ist. Hier muss durch die Zusatzbedingung in den lokalen Matrizen die Summe der Werte multipliziert mit ihren Wichtungsfaktoren auf Stützstellen- und Auswertungsstellen-Seite gleich sein. Diese Forderung entspricht der zusätzlichen Forderung aus Abschnitt 3.5, wenn dort formal $g\left(x_{j}\right)=G\left(y_{j}\right)=1$ gesetzt wird. Unter Benutzung der Bezeichnungen aus Abschnitt 3.5 wird dieses Mal also für jede Zelle $\Omega_{k}$ eine Funktion $s_{k}$ der Form

$$
s_{k}=\alpha_{0} \sum_{j=1}^{N^{(k)}} w_{k}\left(x_{j}^{(k)}\right) \Phi\left(\cdot, x_{j}^{(k)}\right)+\sum_{j=1}^{M^{(k)}} \alpha_{j} \Phi\left(\cdot, y_{j}^{(k)}\right)+p^{(k)}
$$


gesucht, die die Interpolationsbedingungen

$$
\begin{aligned}
s_{k}\left(y_{j}^{(k)}\right) & =F\left(y_{j}^{(k)}\right), \quad 1 \leq j \leq M^{(k)} \\
\sum_{j=1}^{N^{(k)}} w_{k}\left(x_{j}^{(k)}\right) s_{k}\left(x_{j}^{(k)}\right) & =\sum_{j=1}^{M^{(k)}} w_{k}\left(y_{j}^{(k)}\right) F\left(y_{j}^{(k)}\right)
\end{aligned}
$$

sowie die Nebenbedingungen

$$
\alpha_{0} \sum_{j=1}^{N^{(k)}} w_{k}\left(x_{j}^{(k)}\right) q\left(x_{j}^{(k)}\right)+\sum_{j=1}^{M^{(k)}} \alpha_{k} q\left(y_{j}^{(k)}\right)=0, \quad q \in \Pi_{m-1}\left(\mathbb{R}^{d}\right)
$$

erfüllt. Die eindeutige Existenz dieser lokalen Interpolanten folgt aus Theorem 2.3. Dann wird wie in Abschnitt 3.5 noch nachgerechnet, dass die globale Funktion $s=\sum w_{k} s_{k}$ die verallgemeinerten Interpolationsbedingungen erfüllt.

Theorem 3.7 Sei $\Phi$ bedingt positiv definit der Ordnung $m$. Es gelte, dass für $1 \leq k \leq n$ die Menge $Y^{(k)}$ unisolvent bzgl. $\Pi_{m-1}\left(\mathbb{R}^{d}\right)$ ist und dass $X^{(k)}$ nicht in $Y^{(k)}$ enthalten ist. Dann gibt es für $1 \leq k \leq n$ genau eine Funktion $s_{k}$ der Form (2.23), die die Bedingungen (2.24) und (2.25) erfüllt. Die globale Funktion $s:=\sum s_{k} w_{k}$ erfüllt dann die verallgemeinerten Interpolationsbedingungen $s\left(y_{j}\right)=F\left(y_{j}\right), 1 \leq j \leq M$ und

$$
\sum_{j=1}^{N} s\left(x_{j}\right)=\sum_{j=1}^{M} F\left(y_{j}\right)
$$

Wiederum wird neben der globalen Konservativität zusätzlich für jede Zelle $\Omega_{k}$ eine lokale, gewichtete Konservativität über die zweite Bedingung in (2.24) erhalten.

\subsection{Erhaltung der Arbeit und Konservativität}

Transformation der Kräfte mit der transponierten Kopplungsmatrix. Für die transponierte Matrix ist der Beweis bereits durch die beiden Beweise für Konservativität und Arbeitserhaltung geführt.

Zusatzbedingung in der Interpolation mit radialen Basisfunktionen. Durch Aufstellung der Bedingungen zu Konservativität und Arbeitserhaltung in einem Gleichungssystem, so wie sie zuvor einzeln gefordert worden sind, werden beide Bedingungen erfüllt. 


\subsection{Rotationen}

Bei der Verwendung von Rotationen als Zusatzbedingungen (Abschnitt 2.4) in den Interpolationen wird der Hauptteil der zu invertierenden Matrix sowie der resultierenden Kopplungsmatrix 36 mal so groß wie der Hauptteil der Matrizen für die komponentenweise Interpolation ohne Einbeziehung der Rotationen bei gleicher Anzahl von Stützstellen und Auswertungspunkten. So wird hier schon bei einer geringeren Anzahl von Stützstellen ein Verfahren zur Verringerung der Rechenzeit und des Speicherplatzbedarfs notwendig.

Neben den in den vorherigen Abschnitten bereits benutzten Bezeichnungen kommen jetzt wieder noch die Winkel hinzu. Die gegebenen Winkel seien

$$
\theta\left(x_{j}\right)=\left(\theta_{1}\left(x_{j}\right), \theta_{2}\left(x_{j}\right), \theta_{3}\left(x_{j}\right)\right)^{T} \in \mathbb{R}^{3}, \quad 1 \leq j \leq N,
$$

die wieder auf die sich überlappenden Zellen $\Omega_{k}$ aufgeteilt werden. Die Winkel zu den Punkten in $X^{(k)}$ sind demnach

$$
\theta\left(x_{j}^{(k)}\right)=\left(\theta_{1}\left(x_{j}^{(k)}\right), \theta_{2}\left(x_{j}^{(k)}\right), \theta_{3}\left(x_{j}^{(k)}\right)\right)^{T} \in \mathbb{R}^{3}, \quad 1 \leq j \leq N^{(k)},
$$

Im Folgenden werden die Algorithmen zur Interpolation unter Einbeziehung der Rotationen im dreidimensionalen Raum kombiniert mit der „Partition of Unity" Methode vorgestellt. Um die Interpolationsbedingung für die Rotationen global zu erfüllen, muss durch die Verwendung der partiellen Ableitungen in den Interpolanten die Produktregel für die Multiplikation mit den Wichtungsfaktoren beachtet werden. Um dies $\mathrm{zu}$ formulieren ist es sinnvoll wieder die Funktionale aus Abschnitt $2.4 \mathrm{zu}$ benutzen. Dabei ist die folgende Beobachtung wichtig. Alle Funktionale haben Träger in einem Punkt, d.h. sie sind einem Punkt $x_{j}^{(k)}$ zu geordnet. In so einem Fall lässt sich der Zusammenhang zwischen den globalen Funktionalen und den lokalen Funktionalen folgendermaßen, ganz allgemein beschreiben.

Der Einfachheit halber soll zunächst der Fall einfacher, nicht Vektorwertiger Funktionale betrachtet werden. Sind $\lambda_{1}, \ldots, \lambda_{N}$ die global zu rekonstruierenden Funktionale, wobei $\lambda_{j}$ den Träger $x_{j}$ habe, so werden für das lokale Problem auf $\Omega_{k}$ nur die Funktionale in $\Lambda_{k}=\left\{\lambda_{j}: x_{j} \in \Omega_{k}\right\}$ betrachtet. Werden nun zu einem solchen $\lambda_{j}$ das Funktional $\mu_{j}^{(k)}$ durch

$$
\mu_{j}^{(k)}:=\lambda_{j}\left(w_{k} \cdot\right), \quad j \in \Lambda_{k}
$$

definiert und die lokalen Interpolationsprobleme

$$
\mu_{i}^{(k)}\left(s_{k}\right)=\mu_{i}^{(k)}(f), \quad i \in \Lambda_{k}
$$

gelöst, so folgt für die globale Funktion $s=\sum w_{k} s_{k}$ wieder

$$
\begin{aligned}
\lambda_{j}(s) & =\sum_{k=1}^{n} \lambda_{j}\left(w_{k} s_{k}\right)=\sum_{k: x_{j} \in \Omega_{k}} \mu_{j}^{(k)}\left(s_{k}\right)=\sum_{k: x_{j} \in \Omega_{k}} \mu_{j}^{(k)}(f) \\
& =\sum_{k: x_{j} \in \Omega_{k}} \lambda_{j}\left(w_{k} f\right)=\lambda_{j}\left(\sum_{k=1}^{n} w_{k} f\right)=\lambda_{j}(f) .
\end{aligned}
$$


Im konkreten Fall der Rotationen kommt noch erschwerend hinzu, dass die Funktionale und Funktionen vektorwertig sind. Daher ist die Auswertung der $\mu_{j}^{(k)}$ auf eine vektorwertige Funktion $s$ durch die Summe der Auswertung der Komponenten von $\mu_{j}^{(k)}$ auf das Produkt von $w_{k}$ mit den Komponenten von $s$ gegeben:

$$
\mu_{j}^{(k)}(s)=\sum_{i=1}^{d} \lambda_{j, i}\left(w_{k} s_{i}\right)
$$

Hierbei bezeichnet der zweite Index bei $\lambda_{j, i}$ die $i$-te Komponente von $\lambda_{j}$. Theoretisch kann für jede Komponente auch noch eine unterschiedliche Gewichtsfunktion gewählt werden, was hier aber unterbleiben soll. Der Ansatz lässt sich weiter vereinfachen, indem bei den reinen Punktauswertungsfunktionalen die Gewichtsfunktion wieder weggelassen wird. Damit ergeben sich also folgende Funktionale:

$$
\begin{aligned}
\mu_{3 j-\ell}^{(k)} & :=\lambda_{3 j-\ell}^{(k)}, \\
\mu_{3(N+j)-\ell}^{(k)} & :=\lambda_{3(N+j)-\ell}^{(k)}\left(w_{k} \cdot\right)
\end{aligned}
$$

jeweils für $0 \leq \ell \leq 2$ und $1 \leq j \leq N^{(k)}$, wobei die $\lambda_{j}$ durch (2.14) und (2.15) aus Abschnitt 2.4 definiert sind.

Für ein konkretes Beispiel soll hier das Funktional $\mu_{3(N+j)-2}$ betrachtet werden. Da die erste Komponente verschwindet, gilt

$$
\begin{aligned}
\mu_{3(N+j)-2}(s) & =\lambda_{3(N+j)-2,2}\left(w_{k} s_{2}\right)+\lambda_{3(N+j)-2,3}\left(w_{k} s_{3}\right) \\
& =-\frac{\partial\left(w_{k} s_{2}\right)}{\partial w}\left(x_{j}^{(k)}\right)+\frac{\partial\left(w_{k} s_{3}\right)}{\partial v}\left(x_{j}^{(k)}\right) \\
& =\left\{-\left[\frac{\partial w_{k}}{\partial w} s_{2}+\frac{\partial s_{2}}{\partial w} w_{k}\right]+\left[\frac{\partial w_{k}}{\partial v} s_{3}+\frac{\partial s_{3}}{\partial v} w_{k}\right]\right\}\left(x_{j}^{(k)}\right)
\end{aligned}
$$

und dieser Wert muss nun gleich

$$
2 \theta_{3(N+j)-2}\left(x_{j}^{(k)}\right) w_{k}\left(x_{j}^{(k)}\right)
$$

sein.

Um die lokalen Probleme zu lösen, wird wieder der symmetrische Ansatz

$$
s_{k}=\sum_{j=1}^{6 N^{(k)}} \alpha_{j}^{(k)}\left(\mu_{j}^{(k)}\right)^{y}\left(\begin{array}{ccc}
\Phi_{1}(\cdot, y) & 0 & 0 \\
0 & \Phi_{2}(\cdot, y) & 0 \\
0 & 0 & \Phi_{3}(\cdot, y)
\end{array}\right)+p^{(k)}
$$

gewählt und die Interpolationsbedingungen

$$
\begin{aligned}
& \mu_{3 j-2}^{(k)}\left(s_{k}\right)=g_{1}\left(x_{j}^{(k)}\right), \\
& \mu_{3 j-1}^{(k)}\left(s_{k}\right)=g_{2}\left(x_{j}^{(k)}\right),
\end{aligned}
$$




$$
\begin{aligned}
\mu_{3 j}^{(k)}\left(s_{k}\right) & =g_{3}\left(x_{j}^{(k)}\right), \\
\mu_{3(N+j)-2}^{(k)}\left(s_{k}\right) & =2 \theta_{1}\left(x_{j}^{(k)}\right) w_{k}\left(x_{j}^{(k)}\right), \\
\mu_{3(N+j)-1}^{(k)}\left(s_{k}\right) & =2 \theta_{2}\left(x_{j}^{(k)}\right) w_{k}\left(x_{j}^{(k)}\right), \\
\mu_{3(N+j)}^{(k)}\left(s_{k}\right) & =2 \theta_{3}\left(x_{j}^{(k)}\right) w_{k}\left(x_{j}^{(k)}\right)
\end{aligned}
$$

für $1 \leq j \leq N$ sowie die Zusatzbedingungen

$$
\sum_{j=1}^{6 N^{(k)}} \alpha_{j} \mu_{j}^{(k)}(q)=0, \quad q=\left(q_{1}, q_{2}, q_{3}\right), q_{i} \in \Pi_{m-1}\left(\mathbb{R}^{d}\right)
$$

gefordert.

Um ein konkretes Interpolationsproblem zu lösen, muss wiederum die Matrix zur Bestimmung der Koeffizienten $\alpha^{(k)} \in \mathbb{R}^{6 N^{(k)}}$ und der von $p^{(k)}$ aufgestellt und invertiert werden. Diese symmetrische Matrix zerfällt wieder in mehrere Blöcke, die aus der unterschiedlichen Form der Funktionalen resultieren.

Die bisherigen Überlegungen zeigen, dass die lokalen Matrizen unter den üblichen Voraussetzungen invertierbar sind, und dass die globale Interpolante $s=\sum s_{k} w_{k}$ die verallgemeinerten Interpolationsbedingungen erfüllt. 


\section{Softwaretechnische Realisierung}

\section{Die Kopplungsbibliothek MpCCI}

\subsection{Konzept}

Lösungsansätze für gekoppelte Probleme. Es gibt zwei grundsätzlich verschiedene Möglichkeiten, die Simulation von gekoppelten Effekten zu realisieren. Eine Alternative ist die numerische Lösung der verschiedenen partiellen Differentialgleichungen unterschiedlicher physikalischer Phänomene in einem einzigen Simulationscode. Dies würde aber bedeuten, dass komplett neue Simulationscodes angepasst auf bestimmte Problemkreise von Spezialisten aller beteiligten physikalischen Fachrichtungen geschrieben werden müssten. Dies hätte allerdings einen extrem hohen Aufwand zur Folge, da die existierenden monodisziplinären Simulationscodes bereits mehrere hunderttausend Zeilen enthalten und in diesem neuen Code keine Funktionalität ausgelassen werden sollte. Darüber hinaus wäre dieser sehr große, monolithische Code hinsichtlich seiner Wartung und Weiterentwicklung ausgesprochen problematisch. Wie sich allgemein an den modernen Softwareentwicklungsverfahren und -methoden der vergangenen Jahre gezeigt hat, geht der Weg immer mehr in Richtung modularem Aufbau, da die großen, nicht modularen Codes sich langfristig sehr schlecht warten und weiterentwickeln lassen. Zudem müssten für einen solchen Ansatz neue Implementierungen in den monodisziplinären Simulationscodes separat nachgezogen werden.

Die zweite Möglichkeit zur Simulation verschiedener, wechselwirkender physikalischer Phänomene ist die Kopplung existierender, hochentwickelter monodisziplinärer Simulationscodes. Bei einem solchen Ansatz sind die Implementierungen der Simulationscodes der einbezogenen Disziplinen nicht verloren und neue Implementierungen in den monodisziplinären Simulationscodes direkt nutzbar. Diese Alternative hat außerdem den unter modernen Softwaregesichtspunkten entscheidenden Vorteil des modularen Aufbaus.

Realisierung. Mit der im BMB+F Projekt COSIMA-V im Institut für Algorithmen und Wissenschaftliches Rechnen (SCAI) der Fraunhofer-Gesellschaft spezifizierten und implementierten parallelen Kopplungsbibliothek MpCCI (Mesh-based parallel Code Coupling Interface) wird ermöglicht, dass mit bestehenden monodisziplinären Simulationsprogrammen lose Kopplungen durchgeführt werden können [22]. MpCCI ist ein leistungsfähiges Tool, welches bei minimalen notwendigen Änderungen der Simulationscodes und einfacher Handhabung durch die Benutzer die Eigenständigkeit der zu koppelnden Software-Werkzeuge bewahrt.

Ein wesentlicher Gesichtspunkt bei der Spezifikation dieser Kopplungsbibliothek [8] war, die Codes so unabhängig wie möglich voneinander zu halten. 
Das Konzept des Projektes sieht MpCCI als ein Tool mit modularem Aufbau, welches die Simulation von Multi-Physics Problemen auch auf verteilten Architekturen ermöglicht.

MpCCI ist eine MPI-basierte Kommunikationsbibliothek, mit der Daten zwischen mehreren Simulationscodes ausgetauscht werden, wobei diese parallelisiert sein können. Jeder Code arbeitet auf seinen eigenen Gittern und muss keine spezifischen Modifikationen für den Code, mit dem er gekoppelt wird, vornehmen.

Die normalen Pre- und Postprozessoren können weiter für die individuellen Codes benutzt werden.

Die Daten können auf übereinstimmenden oder unterschiedlichen Gittern eines in den Simulationscodes an MpCCI spezifizierten Kopplungsgebietes angeordnet sein, welches ein Volumen, eine Fläche oder auch eine Linie sein kann. Die Gitter müssen beispielsweise bei einer Flächenkopplung nicht auf derselben Fläche liegen, sondern die zu koppelnden Gitterflächen können unterschiedliche Abstände voneinander haben und sich zum Beispiel durchdringen. Der in einer Kopplung maximale Abstand der Flächen ist einstellbar. In dem allgemeinen Fall unterschiedlicher Gitter werden die Werte, wie schon erwähnt, durch MpCCI vom Sendergitter auf das Zielgitter interpoliert. MpCCI regelt die komplexen Verbindungen zwischen den Gittern der Kopplungsregionen. Die Gitter können strukturiert oder unstrukturiert sein und aus den unterschiedlichsten Elementtypen bestehen und zwar auch innerhalb eines Gitters. Im Prinzip sind alle die Kopplungen verschiedener Gitter möglich, für die entsprechende Interpolationen existieren.

Philosophie. Die Philosophie hinter MpCCI ist, MPI auf ein höheres Niveau zu erheben: MPI ermöglicht die Kommunikation zwischen einzelnen Prozessen, MpCCI ermöglicht Datentransfer zwischen zwei oder mehr parallelen Codes. Die MpCCI-Funktionen gleichen den entsprechenden MPIFunktionen so gut wie möglich. Um MpCCI offen für neue Codes zu halten, enthält MpCCI keine spezifische Programmierung für bestimmte Simulationscodes. Mit MpCCI können beliebige Kopplungsalgorithmen aufgesetzt werden. Darüber hinaus werden Standard-Kopplungsalgorithmen mit dem „Synchronisationspunkte-Konzept“ so unterstützt, dass die Kopplung an neue Codes besonders erleichtert wird. Bei diesem Konzept werden in den Codes Punkte spezifiziert, an denen Daten ausgetauscht werden können.

MpCCI wird zu den Simulationscodes gelinkt und die Applikationen rufen MpCCI Funktionen auf, um die gewählten Kopplungsalgorithmen zu realisieren. Die Grundkomponente ist der transparente Austausch von an unterschiedlichen Gittern angeordneten Werten innerhalb einer Kopplungsregion durch MpCCI-Bibliotheksaufrufe.

Die für die gekoppelten Berechnungen verwendeten Simulationscodes sind unter anderem kommerzielle, industrielle Codes, deren Quellcodes nicht für die Integration der Kopplungsfunktionen zur Verfügung stehen. Dies hat zur Folge, dass Co-Prozesse geschrieben werden müssen, die die Kopplung stell- 
vertretend für die eigentlichen Simulationscodes realisieren. Bei einigen Simulationscodes besteht dann die Möglichkeit, diese über Bibliotheksaufrufe zu starten und Werte auszutauschen. Im ineffizientesten Fall werden über eine dateibasierte Lösung die Werte zwischen Co-Prozess und Simulationscode ausgetauscht.

\subsection{Layer-Struktur}

Ausführungspfad. Um ein Verständnis von MpCCI zu vermitteln, wird zunächst die Layer-Struktur einer gekoppelten Anwendung mit MpCCI erklärt: Jeder Prozess der gekoppelten Anwendung hat seinen eigenen MpCCI-Layer und kommuniziert über MpCCI und MPI mit den anderen Prozessen. MpCCI liegt wie ein Layer unterhalb jedes einzelnen Prozesses auf dem MPI-Layer, da MpCCI die MPI-Bibliothek zum Datenaustausch zwischen den Prozessen der verschiedenen Simulationscodes verwendet. Die gesamten Informationen, die benötigt werden, um die Kommunikation über das zugrundeliegende MPI System zu realisieren, sind in den MpCCI-Layern gespeichert.

Der Prozess kann entweder eine MPI-basierte Bibliothek oder MPI selber für die Kommunikation zwischen den Prozessen des eigenen Codes nutzen. Der Code, MpCCI und MPI operieren seriell nacheinander in einem Strang für einen Prozess. Es gibt keine parallelen Aktivitäten zwischen ProzessLayer, MpCCI-Layer und MPI-Layer. Wenn der Prozess seine internen Berechnungen leistet, liegt der MpCCI-Layer, ebenso wie der MPI-Layer, passiv im Hintergrund. MpCCI- oder MPI-Layer werden nur aktiv, wenn der Ausführungspfad durch sie hindurch läuft (siehe Abbildung 3).

Die Prozesse müssen also explizit die MpCCI-Funktionen aufrufen, um die Möglichkeiten von MpCCI zu nutzen. In Abbildung 4 sind schematisch einige wichtige Funktionen dargestellt, mit denen der Code auf MpCCI oder MPI zugreift.

Parallelisierungstechniken. Die Kommunikation zwischen den Prozessen eines einzelnen Codes wird ebenso wie bisher durchgeführt, die MPI Aufrufe bleiben die selben. Die Parallelisierungstechnik, die für die interne Kommunikation eines Codes benutzt wird, hat keinen Einfluss auf die Kopplung der Codes. MpCCI erwartet keinerlei spezifische Parallelisierungstechnik von den Simulationscodes. An einer gekoppelten Anwendung können Codes mit unterschiedlichen Parallelisierungen partizipieren, wie in Abbildung 5 als ein Beispiel zu sehen ist. Nur eine Untermenge der Prozesse eines Codes muss an der Kopplung beteiligt sein.

Dies ist z.B. sinnvoll für Parallelisierungsstrategien, bei denen einige Prozesse nur Unteraufgaben erfüllen und den allgemeinen Berechnungsalgorithmus nicht kennen und so auch nicht an der Kopplung beteiligt sein müssen. Trotzdem ist es notwendig, dass alle Prozesse der Simulationscodes an der MpCCI-Initialisierung und der Beendigung der gekoppelten Berechnung durch MpCCI teilnehmen. In Abbildung 5 sind alle Prozesse von Code A, der eine 


\section{Prozess der gekoppelten Anwendung}

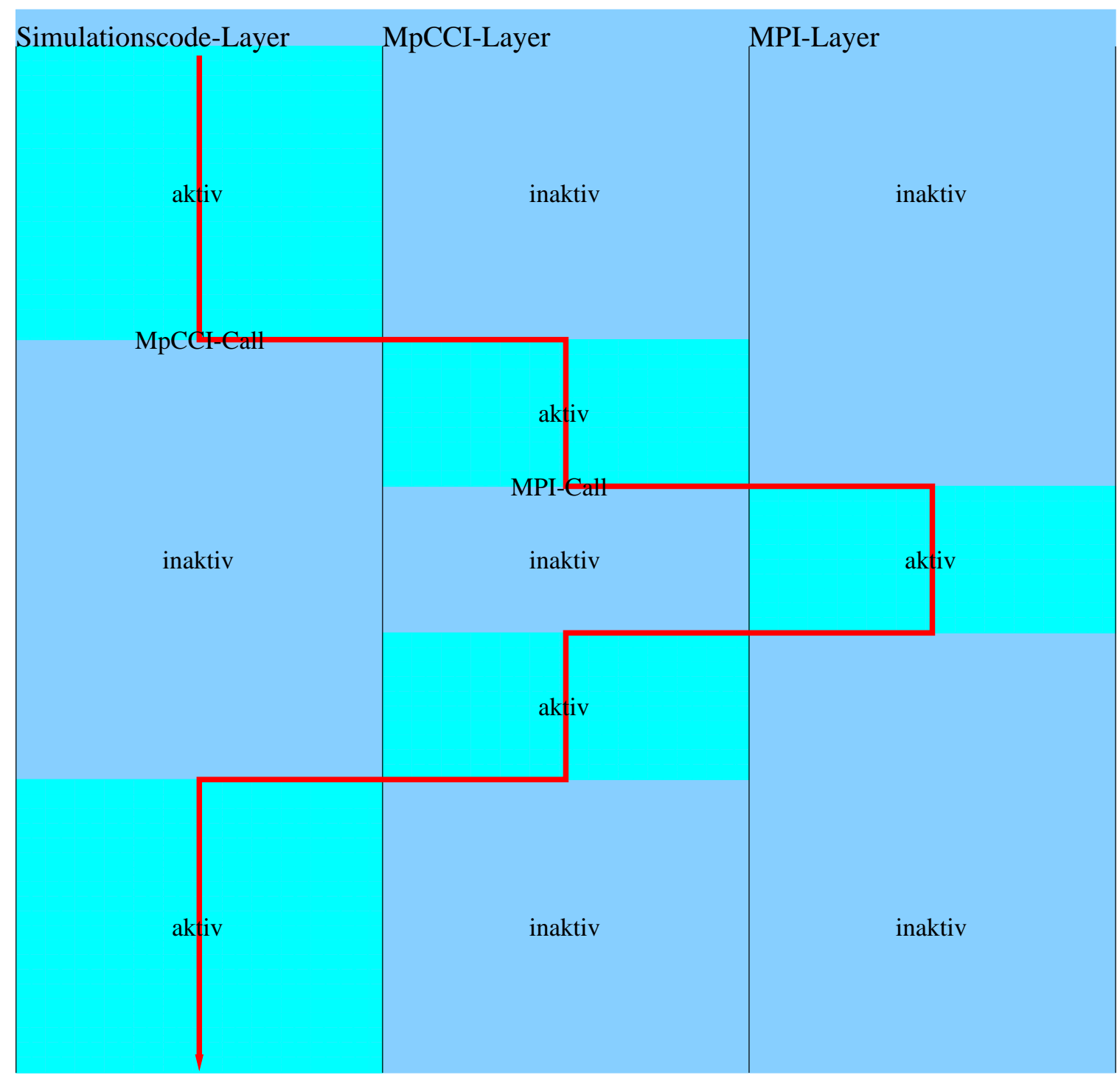

Abbildung 3. Das Beispiel zeigt die Abfolge der aktiv werdenden Layer bei einem MpCCI-Call, der eine Kommunikation durchführt.

Prozess von Code B und zwei Prozesse von Code C an der Kopplung beteiligt, also MpCCI-Prozesse. Die Abbildung zeigt auch die Kommunikation zu dem optionalen Kontrollprozess.

MPI hat mit einem Kommunikator-Konzept die Fähigkeit den Kommunikationsraum aufzuteilen. Dies ermöglicht den parallelen Codes, an der gekoppelten Simulation zu partizipieren, ohne miteinander zu interferieren. Darüber hinaus ist die Kommunikation mit MpCCI komplett getrennt von der internen Kommunikation der Simulationscodes.

Codespezifische Aufgaben in der Kopplung können getrennt von den generellen Aufgaben der Kopplungsbibliothek realisiert werden. Das codespezifische Interface kann als ein Layer von Unterroutinen auf der Basis von 


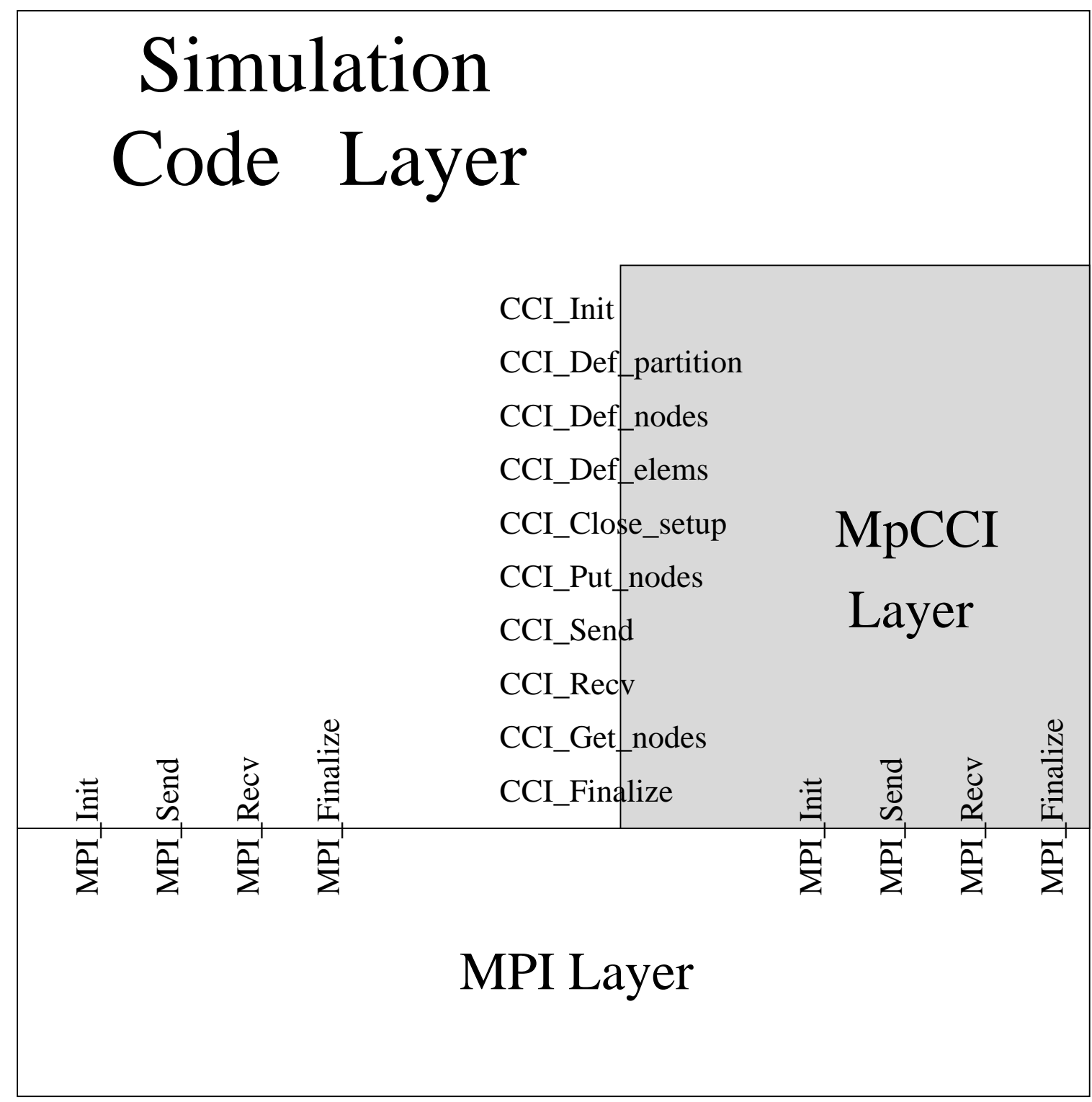

Abbildung 4. Die Abbildung zeigt die Layer eines Prozesses mit den Zugriffen der Layer auf die wichtigsten Funktionen des jeweils anderen Layers.

MpCCI implementiert werden. Der Aufwand, separat entwickelte Codes zu koppeln, ist durch diese Flexibilität minimiert.

\subsection{Kopplungsphasen}

Erläuterung der Kopplungsphasen. Zum besseren Verständnis wird ein Ablaufdiagramm gezeigt, welches die Hauptphasen der Kopplung mit MpCCI darstellt. Es werden darin nur zwei Simulationscodes gekoppelt, um eine bessere Übersichtlichkeit zu erreichen. Das Konzept ist aber anwendbar auf beliebig viele Simulationscodes. Die Ablaufschritte sind stark zusammengefasst, da nur auf die Phasen fokussiert werden soll. 


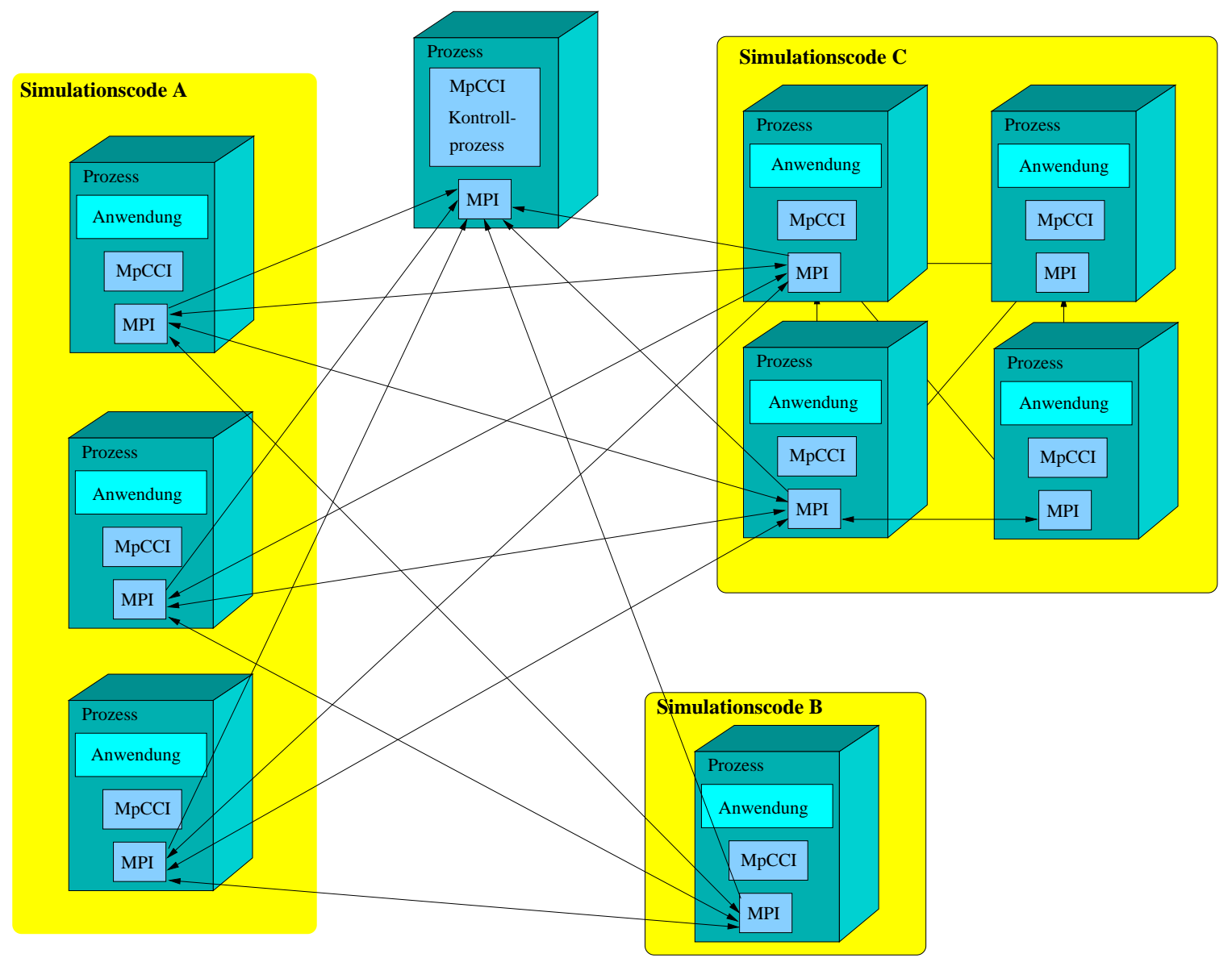

Abbildung 5. In diesem Beispiel sind verschiedene Parallelisierungstechniken in einer Simulation gekoppelt.

Eine gekoppelte Simulation mit MpCCI lässt sich in vier Phasen aufteilen. Der Kopplungsablauf beginnt mit der Startup-Phase. In der Phase wird ein serieller Prozess gestartet, der über die Angaben in seinem MpCCI-Inputfile die Prozesse der Simulationscodes auf den jeweiligen physikalischen Prozessoren startet. Die Simulationscodes lesen dann ihre eigenen, zuvor über PreProzessoren generierten, Inputfiles. Das MpCCI-Inputfile gibt an, wie die zu koppelnden Simulationscodes gestartet werden. Die Standard-Umgebungen und Kopplungsgrößen sind ebenfalls im MpCCI-Inputfile spezifiziert. Es beschreibt die Art der Kopplungsgrößen, die zu verwendende Interpolation, gegebenenfalls den Kopplungsalgorithmus und ob und auf welche Weise, für die Kopplung benötigte Eigenschaften untersucht werden sollen. Das Inputfile legt außerdem fest, welche Kopplungsgrößen jedes Codes aufeinander abgebildet werden sollen. Die codespezifischen Teile des Inputfiles, wie die Standardumgebungen der Simulationscodes, der Bezug zu den Kopplungsgrößen und wie sie aufeinander abgebildet werden, kann auch in separate Konfigurationsfiles geschrieben werden, die dann in das Inputfile eingebunden werden.

In der Startup-Phase müssen die Prozesse der Simulationscodes CCI_Init aufrufen, um die gekoppelte Berechnung aufzunehmen. Es werden Kommuni- 


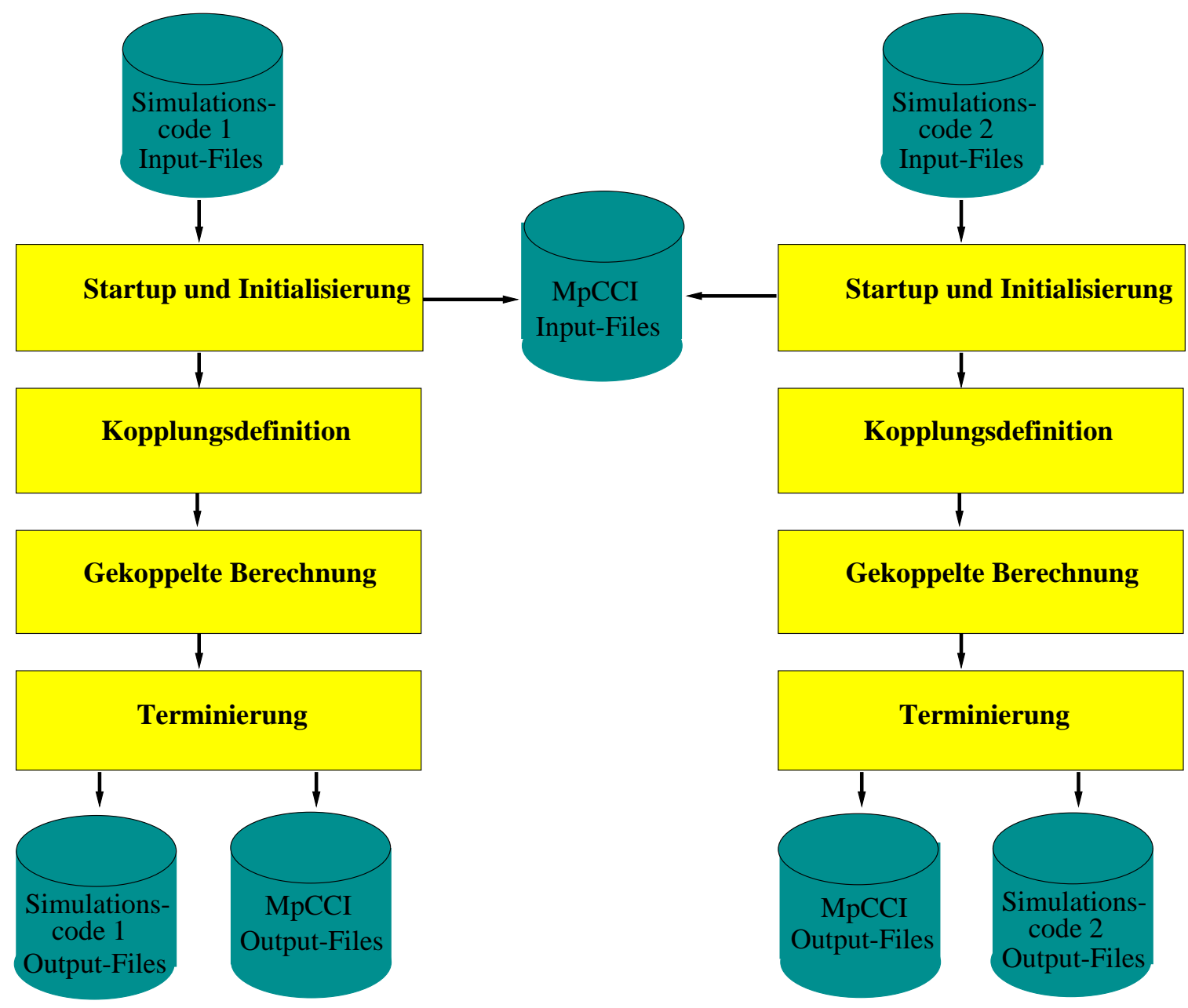

Abbildung 6. Die Abbildung zeigt die Abfolge der Kopplungsphasen.

katoren für die verschiedenen Arten von Kommunikationen aufgebaut. Zum einen für die Kommunikation der Prozesse eines Simulationscodes untereinander, zum anderen für die Kommunikation der MpCCI-Prozesse eines Simulationscodes mit denen eines anderen Simulationscodes. Zu diesem Zeitpunkt wird auch, über die Angaben im MpCCI-Inputfile, das Environment für einen Code gesetzt, und die Kommunikation zwischen den verschiedenen Prozessen über die MpCCI-Layer wird errichtet.

Die verschiedenen Teile einer Kopplungsfläche auf einem Prozess werden als Partitionen bezeichnet. Die Bezeichnungen der an MpCCI zu definierenden Gitterteile ist in Abbildung 7 skizziert. Es dürfen mehrere Gitter an MpCCI definiert werden, um für Simulationscodes, die auf mehreren Gittern arbeiten (z.B. original und duales Gitter) auch Kopplungen zwischen mehreren Gittern zu ermöglichen, wie in Abbildung 8 dargestellt.

In der Kopplungs-Definitions-Phase definiert jeder der MpCCI-Prozesse eines Simulationscodes durch den Aufruf von CCl_Def_partition alle Partitionen jedes Gitters, mit welchem er hinsichtlich der Kopplung arbeiten möchte. Dann werden die Knoten für jede dieser Partitionen mit Hilfe von CCI_Def_nodes an MpCCI spezifiziert. Der Funktionsaufruf CCI_Def_elems be- 


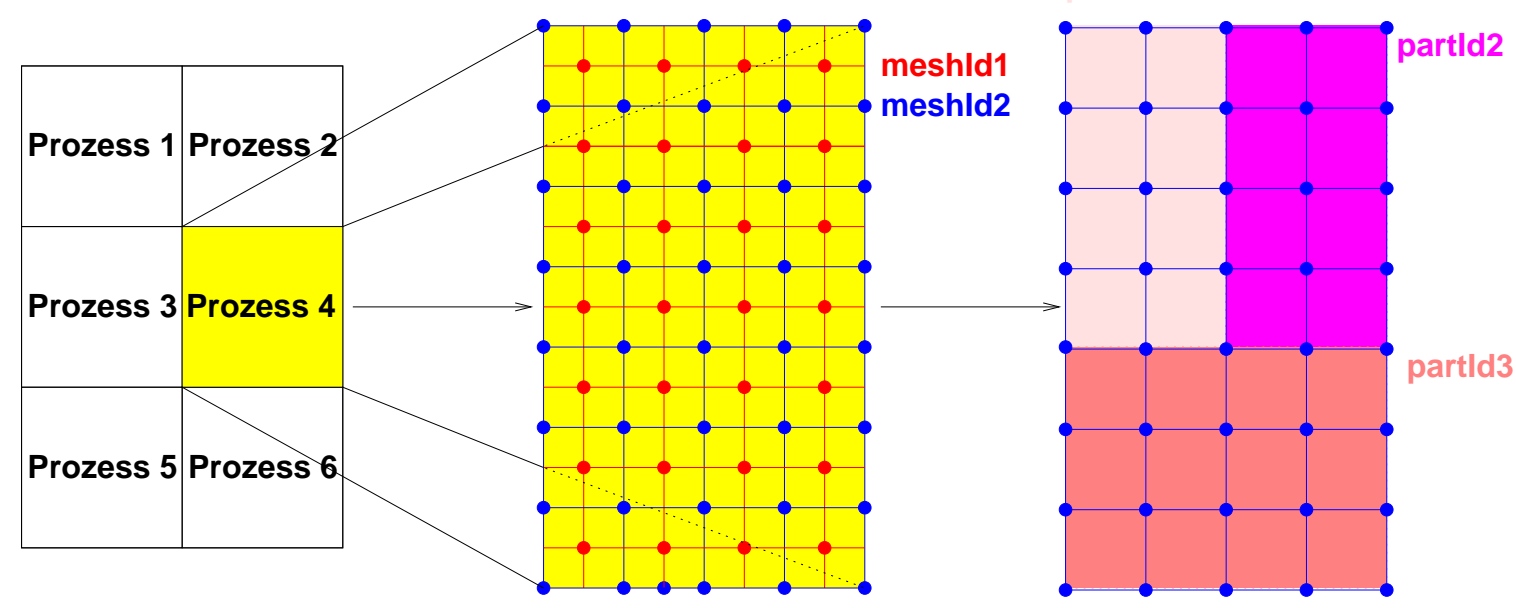

Abbildung 7. Auf jedem Prozess können mehrere Gitter jeweils mit unterschiedlichen „meshlds“ spezifiziert werden, die global über alle Prozesse eines Simulationscodes eindeutig sein müssen. Diese Gitter können auf jedem Prozess in mehrere Partitionen aufgeteilt sein, für die jeweils eine prozesslokale „partld “ definiert werden muss.

\section{Simulationscode 1}

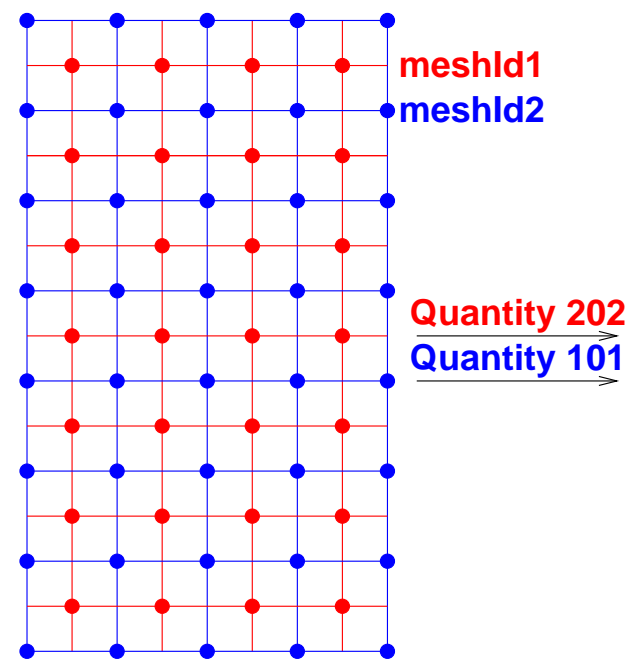

\section{Simulationscode 2}

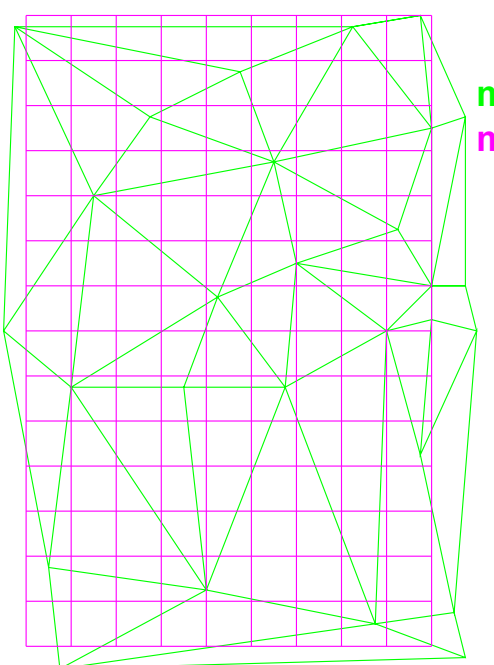

meshld1 meshld

Abbildung 8. Es können mehrere Gitter auf den Simulationscodes in die Kopplung einbezogen werden und beliebige Paarungen dieser Gitter für den Austausch der Daten gebildet werden. In dem gezeigten Beispiel wird die Größe mit der Bezeichnung „Quantity 202“ von dem Gitter mit der „meshld 1" des Simulationscodes 1 auf das Gitter mit der "meshld 1" des Simulationscodes 2 interpoliert und unter der Größe mit der Bezeichnung „Quantity 44" abgespeichert. Die Größe mit der Bezeichnung „Quantity 101“ wird von dem Gitter mit der „meshld 2" des Simulationscodes 1 auf das Gitter mit der „meshld 2" des Simulationscodes 2 interpoliert und unter der Größe mit der Bezeichnung „Quantity 33“ abgelegt. Die „meshld“ ist innerhalb eines Simulationscodes eindeutig.

wirkt, dass in MpCCI die Elemente des Gitters für jede Partition an Hand 
der Knotennummerierung festgelegt werden. Danach wird durch die Funktion CCl_Close_setup die Nachbarschaftssuche und die Vorbereitung der Interpolation angestoßen. Mit dieser Funktion werden auch die Prozesse spezifiziert, welche sich weiter an der Kopplung über MpCCI-Funktionsaufrufe beteiligen werden. Diese Prozesse der Simulationscodes werden als MpCCI-Prozesse bezeichnet. Die MpCCI-Prozesse eines Simulationscodes können eine nicht leere Untergruppe der Prozesse des Simulationscodes bilden (siehe Abbildung 9). Die MpCCI-Instanz, die für die an der Kopplung nicht beteiligten Prozesse aufgebaut wird, ist entsprechend klein. Sie enthält nur ein Gerüst der aktiven Instanz. Die Kommunikation zwischen den MpCCI-Prozessen ist so direkt wie möglich; die Nachbarschaftsbeziehungen regeln, ob zwischen zwei Prozessen eine Kommunikation stattfindet oder nicht.

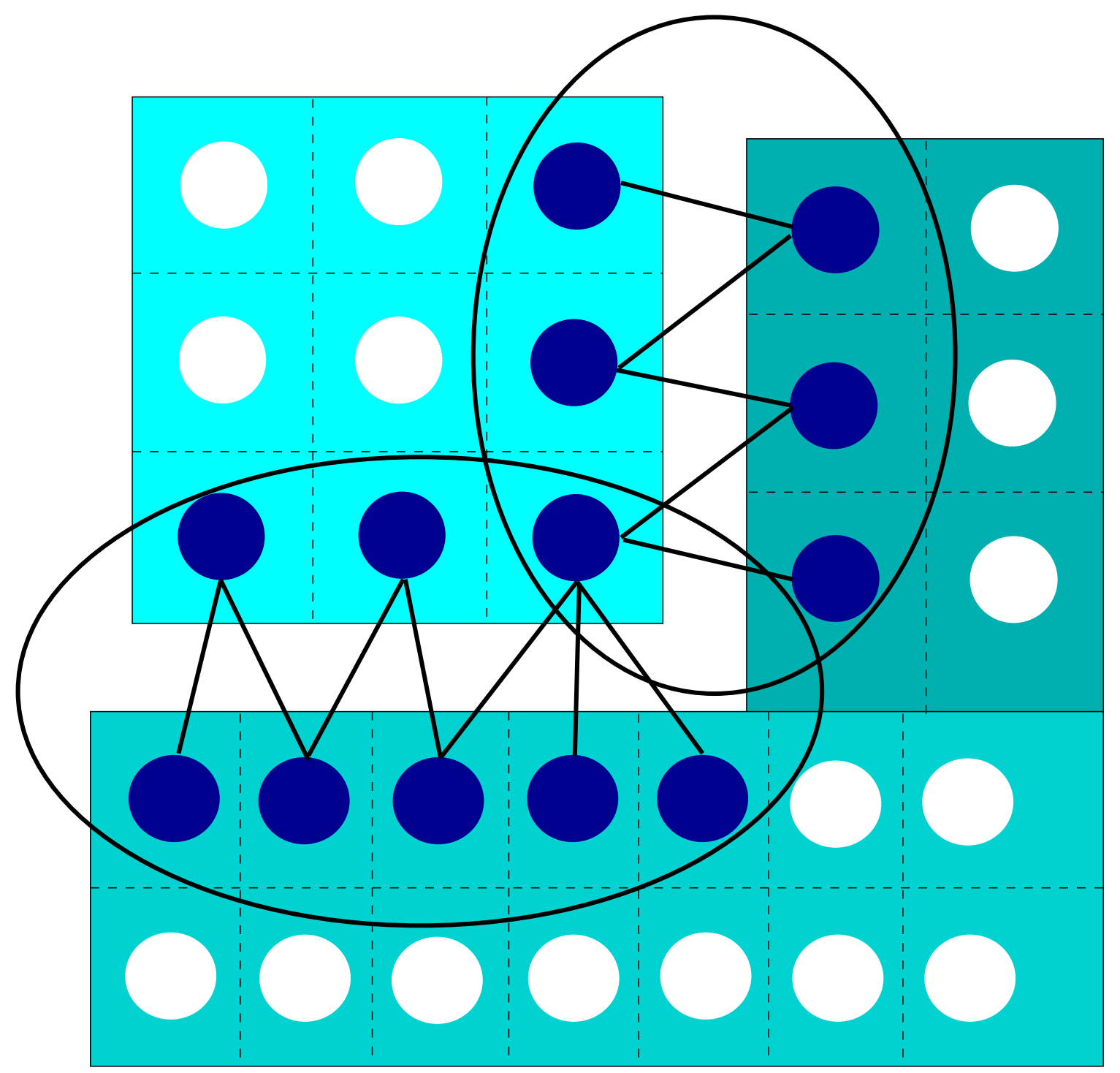

Abbildung 9. Es sind nur Teilmengen der Prozesse von den Codes an der Kopplung beteiligt. In diesem Beispiel sind zwei Gruppen von Prozessen zu sehen, die getrennt voneinander kommunizieren mit jeweils eigenem MPI-Kommunikator. 
Mit der Funktion CCl_Close_setup wird die Kopplungs-Definitions-Phase abgeschlossen.

In der eigentlichen Kopplungsphase gibt jeder MpCCI-Prozess des SenderSimulationscodes seinen lokalen Teil der zuvor im Simulationscode berechneten Kopplungswerte auf den Knoten bzw. Elementzentren mit CCI_Put_nodes bzw. CCI_Put_elems an die Kopplungsbibliothek. Bei MpCCI wird grundsätzlich unterschieden, ob die Werte an den Knoten oder Elementzentren angeordnet sind. Diese Werte werden dann mit CCl_Send an den jeweiligen, vorher in den Nachbarschaftsberechnungen in MpCCI bestimmten Prozess des spezifizierten Empfängercodes geschickt und dort mit CCI_Recv empfangen. Die Kommunikation ist durch die Informationen aus der Nachbarschaftssuche so angepasst, dass die Daten nur zu den Prozessen geschickt werden, wo sie auch benötigt werden, und nicht zu allen Prozessen des Empfängercodes.

Die vorrangige Aufgabe von CCl_Send/CCl_Recv ist die transparente Kommunikation zwischen den MpCCI-Prozessen der Simulationscodes.

Im Inputfile wird spezifiziert, wie schon oben beschrieben, nach welchem Algorithmus eine Kopplungsgröße interpoliert werden soll. Danach richtet sich z.B., ob sie unter der Bedingung der Konservativität interpoliert werden muss oder nicht. Auf der Senderseite wird beim Funktionsaufruf CCCISend interpoliert, wenn im Inputfile eine Standardinterpolation ohne Bedingung der Konservativität spezifiziert worden ist. Bei einer eingestellten Standardinterpolation unter Bedingung der Konservativität verhält es sich genau umgekehrt, die Werte werden erst zum Empfängerprozess geschickt und dann wird in der $\mathrm{CCl}$ _Recv-Routine interpoliert. Dies liegt daran, dass je nach Interpolation die Quell- oder Zielgittertopologie benötigt wird.

Die Funktionen CCl_Get_nodes bzw. CCl_Get_elems verteilen dann je Prozess lokal die Werte wieder an die entsprechend angegebenen Knoten bzw. Elementzentren. Der Empfängercode kann daraufhin die Berechnungen starten, die die Daten aus der Kopplung benötigten. Dieser Teil der gekoppelten Anwendung, welcher die eigentliche Berechnungsschleife beinhaltet, ist die Phase, in der der Kopplungsalgorithmus aufgesetzt wird.

In der Terminationsphase wird die gekoppelte Berechnung durch den Aufruf der Funktion $\mathrm{CCl}_{-}$Finalize in jedem Prozess der gekoppelten Codes beendet. Nach diesem Aufruf darf keine erneute Initialisierung gestartet und keine MpCCI-Funktion aufgerufen werden.

Eine gekoppelte Anwendung kann optional mit einem Kontrollprozess gestartet werden. Der Kontrollprozess kann bei einer Kopplung mit MpCCI verwendet werden, um die gekoppelte Berechnung zu überwachen, bis alle Prozesse aller Simulationscodes eine Meldung über die Beendung der gekoppelten Berechnung gesendet haben, wie in Abbildung $6 \mathrm{zu}$ sehen ist. Diese „Monitoring Function“ bewirkt z.B. in dem Fall eines unerwarteten Programmabbruchs eines Simulationscodes, dass der Kontrollprozess dieses registriert und den anderen Simulationscodes eine entsprechende Fehlermeldung zusendet. In einem Debug Mode kontrolliert der Kontrollprozess die parallele Berechnung und gibt Warnungen bei möglichen „Dead Lock“ Situa- 
tionen aus. Der Kontrollprozess kann außerdem benutzt werden, um kollektive Kommunikationsaufgaben zu behandeln.

Beispiel einer Kopplung. In Abbildung 10 ist der Ablauf einer Beispielkopplung mit den entsprechenden MpCCI-Aufrufen dargestellt.

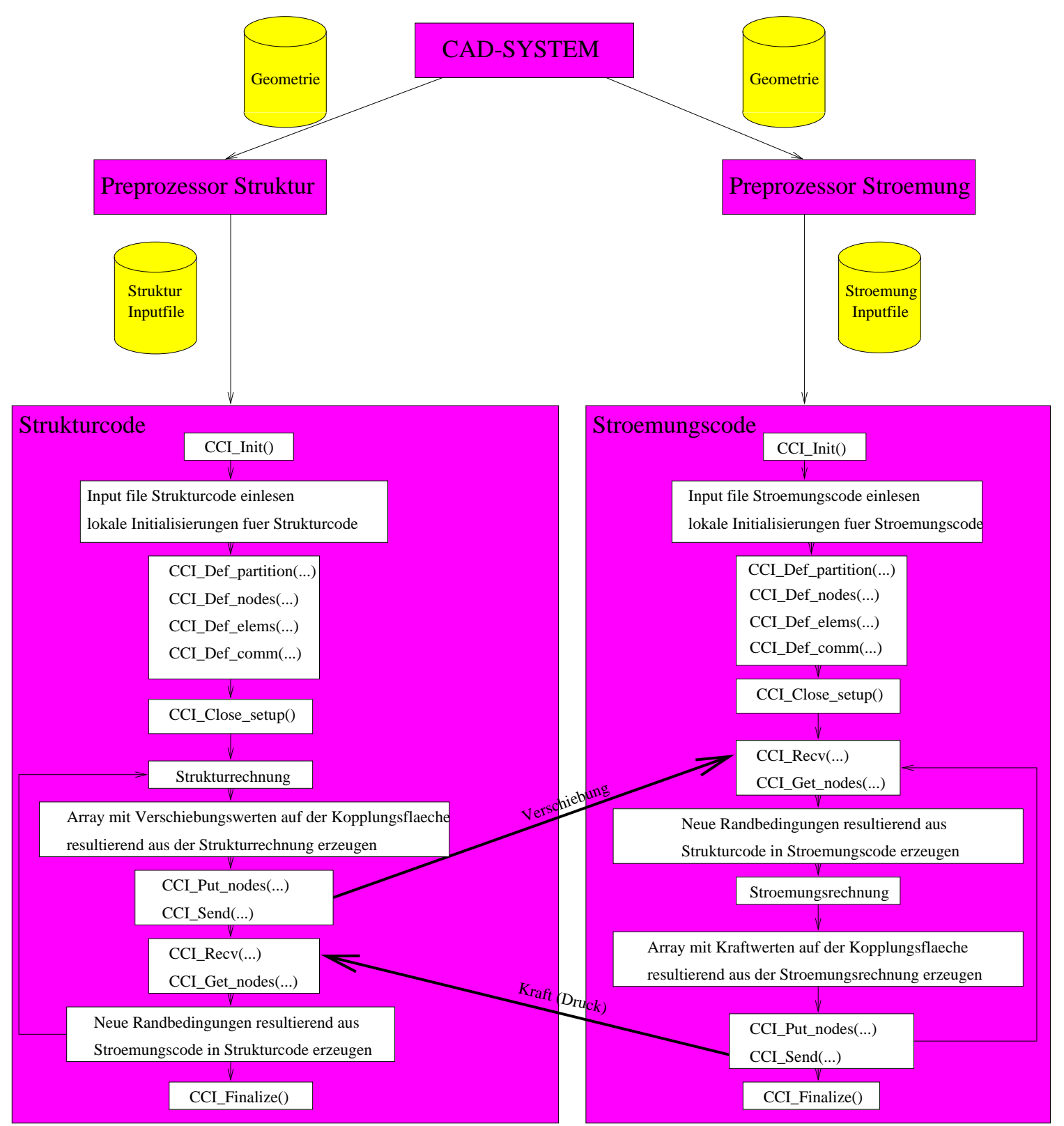

Abbildung 10. In diesem Beispiel werden zwei serielle Codes mit jeweils einer Partition gekoppelt und es wird ein einfacher alternierender Kopplungsalgorithmus aufgesetzt. 


\subsection{Zusätzliche Tools}

MpCCI hat verschiedene Debug Level und Monitor Möglichkeiten, welche an- oder abgeschaltet werden können. Durch ein Playback Tool existiert die Möglichkeit, einen Dummy Code aus den MpCCI Output-Files einer gekoppelten Applikation zu konstruieren, welcher die gleichen MpCCI-Funktionen mit den gleichen Daten aufruft. Darüber hinaus ist ein Visualisierungstool MpCCI-Visualiser verfügbar, welches das bisherige Tool COCOVis [23] zur Visualisierung der Kopplungsgebiete ersetzt. Der MpCCI-Visualiser bietet einen Einblick in die Gitter der gekoppelten Regionen, der Standard-Nachbarschaftsinformationen und der transferierten und interpolierten Werte. Ein anderes zusätzliches Tool von MpCCI ist ein Graphical User Interface (GUI). Das GUI kann bisher dazu benutzt werden, die Parameter einer gekoppelten Simulation zu spezifizieren, welche anderenfalls in dem speziellen MpCCI Inputfile definiert werden müssten. Der Benutzer kann die gekoppelte Simulation mit dem GUI starten und zukünftig werden auch Möglichkeiten zur interaktiven Kontrolle integriert sein.

\subsection{Standardinterpolationen}

Nachbarschaftssuche. Die in MpCCI implementierte Standard-Suchmethode für aufeinander passende und nicht passende Gitter besteht aus vier Schritten: Im ersten Schritt generiert MpCCI Element-Knoten Paare (bei nicht aufeinander passenden Gittern) und Knoten-Knoten Paare (bei aufeinander passenden Gittern), welche für eine detailliertere Suche in Frage kommen. Im nächsten Schritt wird ein detailliertes Kriterium auf alle in Schritt 1 generierten Paare angewendet und das Paar ausgesucht, welches nach dem Kriterium das Beste ist. Wenn es erforderlich ist, startet MpCCI im dritten Schritt eine lineare Suche über alle Knoten, die im ersten Schritt nicht zugeordnet werden konnten, und sucht direkt das beste Paar nach dem detaillierten Kriterium. Im letzten Schritt filtert MpCCI alle Knoten heraus, die nach dem „Rejection" Kriterium keinen Nachbarknoten oder kein Nachbarelement erhalten sollen.

Die Nachbarschaftssuche sowie auch die Interpolation werden hier nur für die Flächenkopplungen im dreidimensionalen Raum beschrieben, da nur diese räumliche Konstellation für die Kopplungen in der Aeroelastik von Interesse ist. Die Nachbarschaftssuche wird im folgenden Text für nicht aufeinander passende Gitter in vereinfachter Weise beschrieben. Für jeden Knoten eines Gitters wird auf allen Gitterpartitionen aller Prozesse des jeweils gegenüberliegenden Simulationscodes das Element unter allen Elementen der Kopplungsfläche gesucht, welches die kürzeste Distanz zu dem Knoten hat. Bei einer Flächenkopplung, welche in dem hier relevanten Zusammenhang alleine von Interesse ist, wird jedes Element zunächst in Dreiecke aufgeteilt.

Um jedes Dreieck wird eine sogenannte „Bounding Box“ konstruiert, die gerade groß genug ist, um alle Knoten des Elementes zu enthalten. Dann werden die „Bounding Boxes“ so vergrößert, dass die größte Breite der „Bounding 
Boxes" aus dem ersten Schritt die Länge in jeder Koordinatenrichtung der neuen „Boxes“ ist. Als nächstes werden die „Bounding Boxes“ noch von ihrem Zentrum aus mit einem einstellbaren Faktor ausgedehnt.

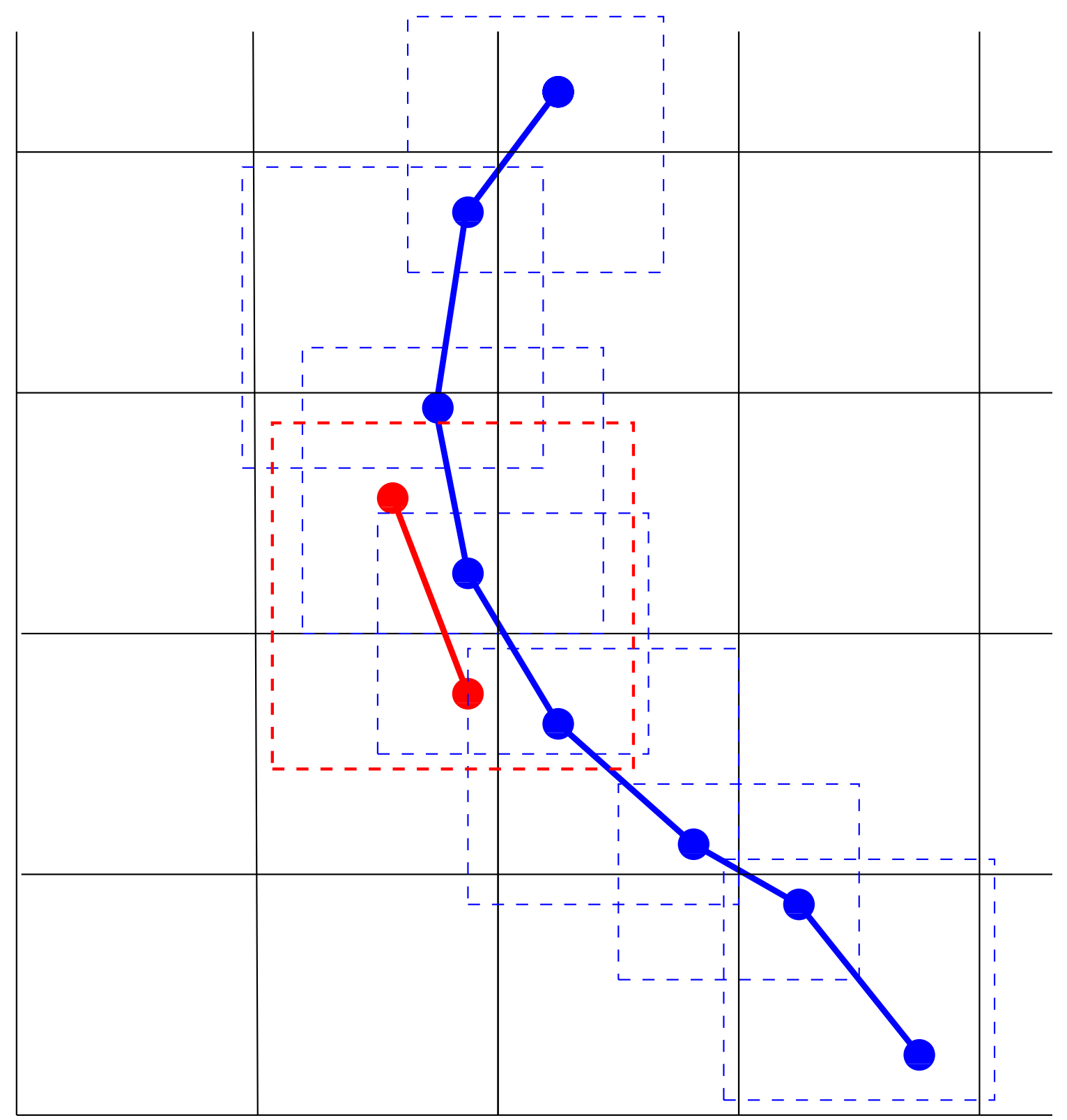

Abbildung 11. Das Kopplungsgebiet wird in „Buckets“ geteilt, das heißt, über das Kopplungsgebiet wird ein reguläres, rechtwinkliges Gitter gezogen. Dann werden den „Buckets“ alle Elemente zugeordnet, deren „Bounding Boxes“ eine Überschneidung mit dem jeweiligen „Bucket“ haben. Die Abbildung zeigt einen Schnitt durch eine Kopplungsfläche. Die „Bounding Boxes“ sind mit gestrichelten Linien um die Elemente gezeichnet.

Im folgenden Schritt berechnet der Algorithmus die Durchschnittsgröße aller konstruierten „Bounding Boxes“. Dann wird ein reguläres, rechtwinkli- 
ges Gitter, ein sogenanntes „Bucket-Gitter“ erzeugt. Der Gitterabstand ist die Durchschnittsgröße der „Bounding Boxes“ multipliziert mit einer variablen Größe. Die Dreieckselemente, deren „Bounding Boxes“ eine nicht leere Schnittmenge mit einem Bucket bilden, werden diesem zugeordnet (siehe Abbildung 11). Ebenso gehören die Knoten zu den „Buckets“ in denen sie jeweils liegen.

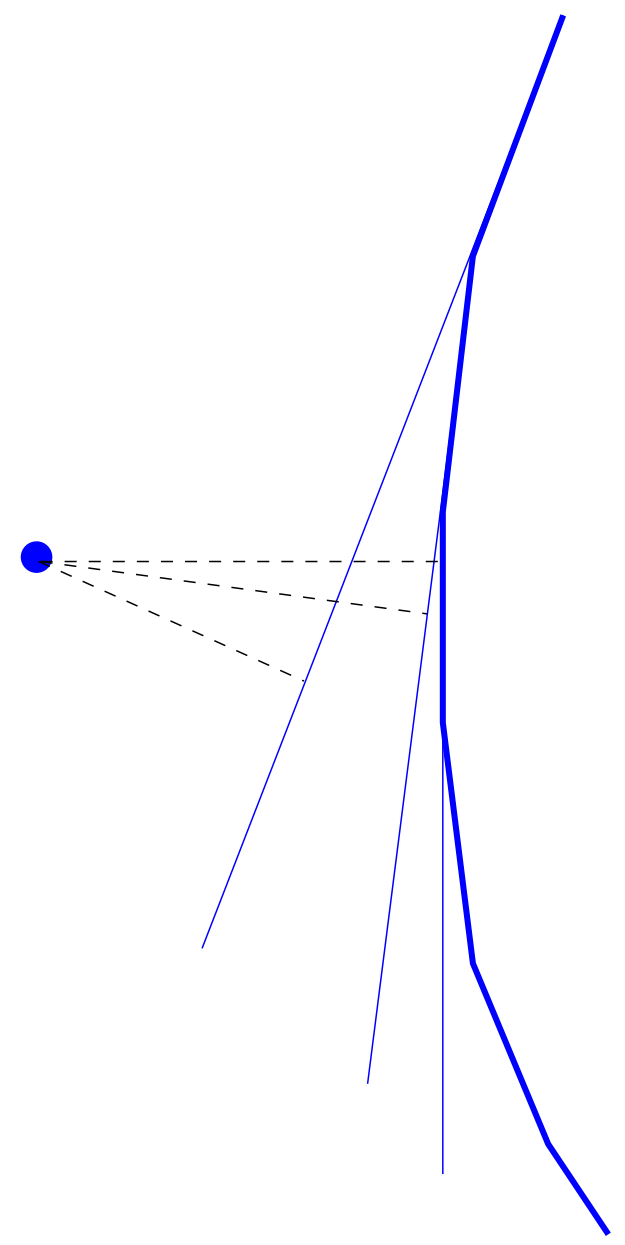

Abbildung 12. Die Knoten werden in die Ebenen der Elemente projiziert.

Um nun das Dreieck zu ermitteln, welches die kürzeste Distanz zu einem bestimmten Knoten hat, muss der Algorithmus nur die Elemente betrachten, die dem „Bucket“ zugeordnet sind, in dem der jeweilige Knoten liegt. In diesem Schritt berechnet MpCCI die kleinste Distanz zwischen dem gefragten Knoten $p$ und allen Punkten jedes der ausgesuchten Dreiecke $\triangle A B C$ des jeweils gegenüberliegenden Gitters. Dafür wird der Knoten in die Ebene jedes der Dreiecke projiziert und der kürzeste Abstand bezüglich des unten angegebenen Kriteriums über baryzentrischen Koordinaten ermittelt. (siehe Abbildung 12 ). 
Das sogenannte „Rejection-Kriterium“ ist durch drei Parameter - $\theta_{1}, \theta_{2}$, $\theta_{3}$ - konfigurierbar. Alle Knoten, für welche

$$
\theta_{1} \cdot(|u|+|v|+|w|-1)+\theta_{2} \cdot \frac{d_{t}^{2}}{|\Delta A B C|}+\theta_{3} \cdot \frac{d_{n}^{2}}{|\Delta A B C|}>\tau
$$

für jedes Dreieck $\triangle A B C$ gilt, erhalten kein Nachbarelement und werden so nicht in die Kopplung einbezogen. Anschaulich definiert das „RejectionKriterium" über einstellbare Parameter einen Bereich um ein Element, in welchem die Knoten dem Element noch zugeordnet werden dürfen.

In (2.26), bezeichnen $u, v$, und $w$ die baryzentrischen Koordinaten der Projektion $p^{\prime}$ des Knoten $p$ auf die Ebene, die durch das Dreieck $\triangle A B C$ definiert wird. Die Abstände zwischen $p$ und dem Projektionspunkt $p^{\prime}$ von $p$ in der Ebene des Dreiecks $\triangle A B C$, sowie zwischen $p^{\prime}$ und dem Punkt $p^{\prime \prime}$ innerhalb des Dreiecks $\triangle A B C$, welcher den minimalen Abstand zu $p$ hat (siehe Abbildung 13), werden mit $d_{n}=\left\|p-p^{\prime}\right\|$ und $d_{t}=\left\|p^{\prime}-p^{\prime \prime}\right\|$ bezeichnet. Die Schwierigkeiten hierbei liegen darin, dass die Kopplungsflächen der

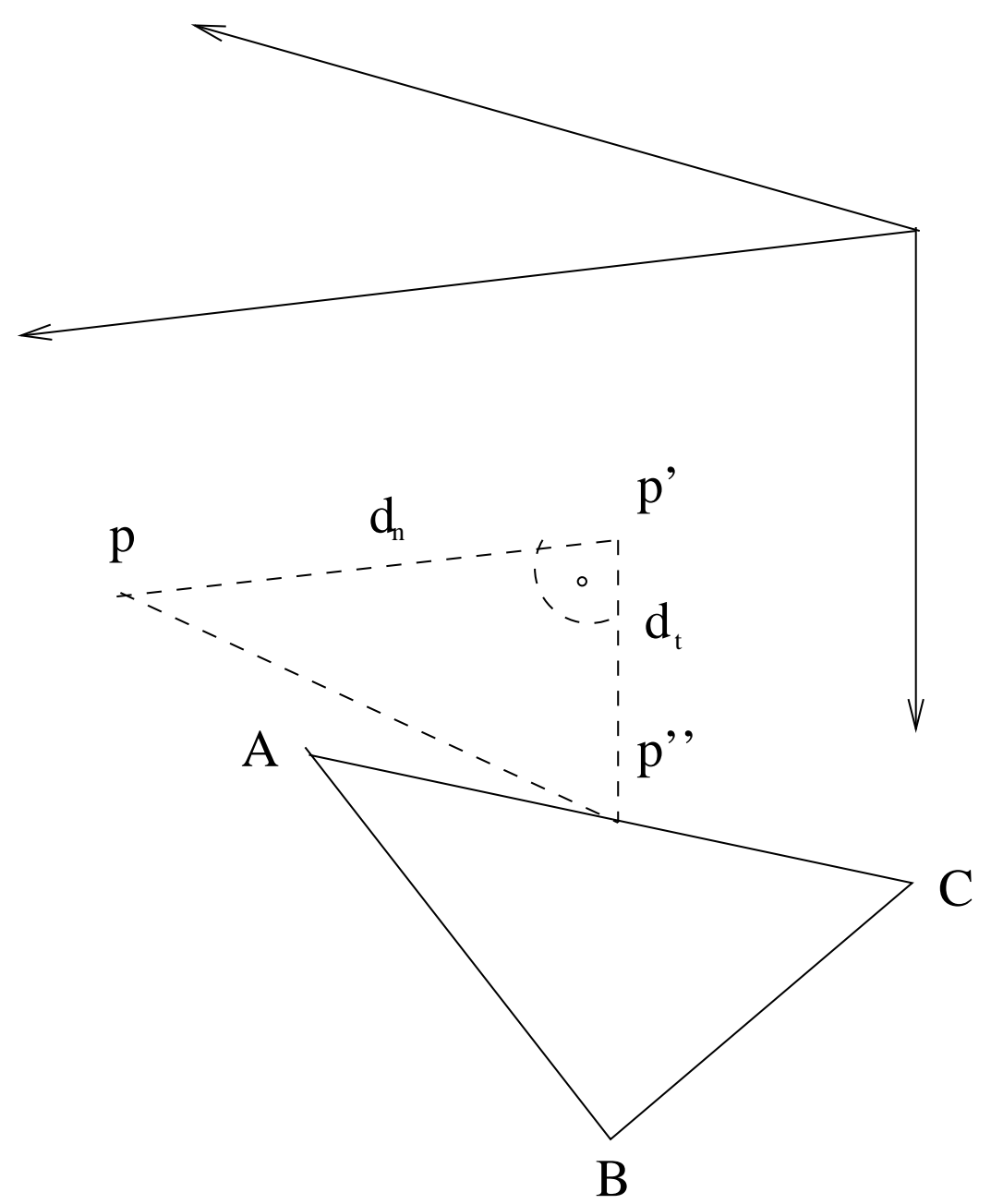

Abbildung 13. Skizze zu dem Rejection-Kriterium. 
gekoppelten Codes auf mehrere Partitionen mehrerer Prozesse verteilt sein können und Abstände und Durchdringungen zwischen den Kopplungsflächen der Codes zugelassen sind.

Interpolation. Wie schon oben begründet wurde, werden nur die Standardinterpolationen für Flächenkopplungen im dreidimensionalen Raum beschrieben. Auf die in MpCCI für diese Art von räumlichen Kopplungen implementierten Interpolationen (siehe [8]), die aber für die in dieser Arbeit behandelten Probleme nicht anwendbar sind, wird ebenfalls nicht eingegangen.

Die Interpolation setzt auf den baryzentrischen Koordinaten des Knotens bezüglich seines Nachbarelementes als Ergebnis der Nachbarschaftssuche auf. Implementiert sind je nach Elementtyp lineare, bilineare und quadratische Interpolationen. Der Benutzer kann jeweils zwischen einer Interpolation mit oder ohne Bedingung der Konservativität wählen. Im folgenden werden die Elementtypen mit ihren jeweiligen Interpolationsformeln aufgelistet (siehe Abbildung 14).
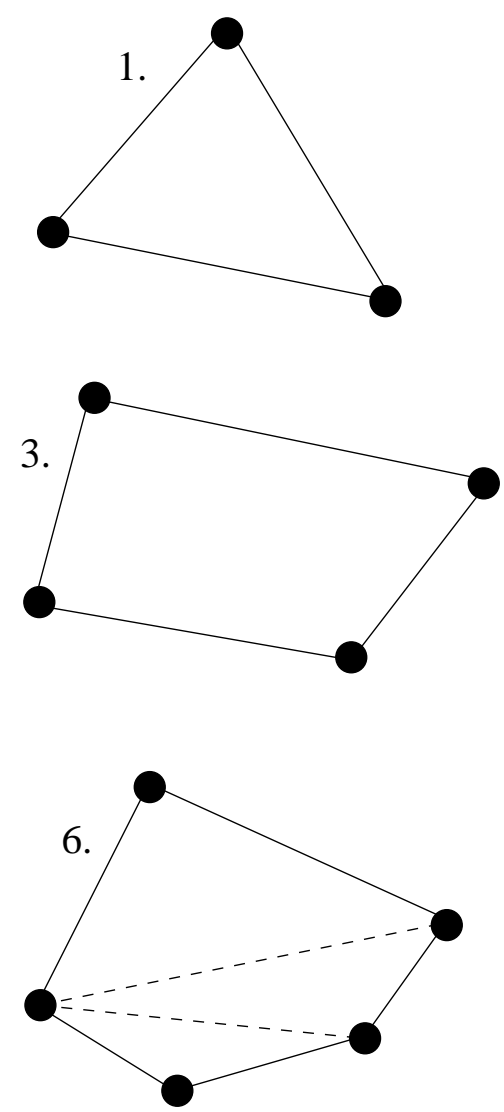

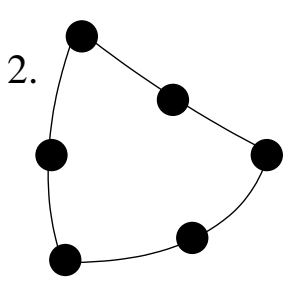

4.
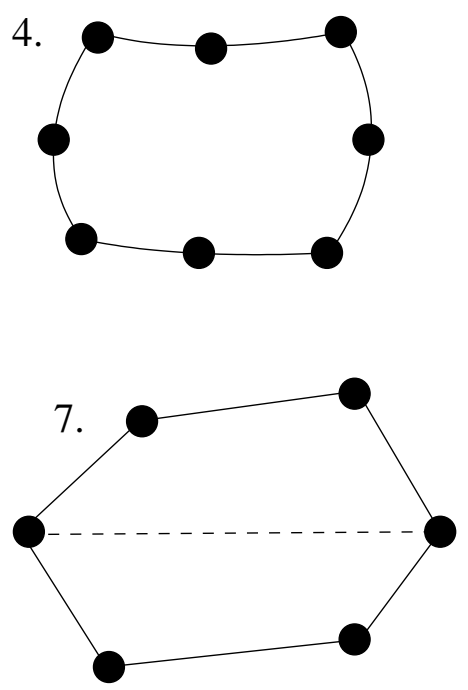

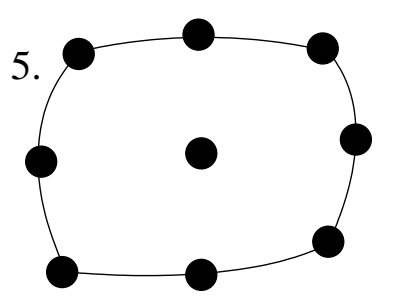

Abbildung 14. Die Interpolationsformeln zu den Elementtypen sind mit der entsprechenden Nummer angegeben. Die Elementtypen mit der Nummer 6 und 7 werden in Dreiecke des Typs 1 bzw. Vierecke des Typs 3 zerlegt. 
1.

$$
G(u, v)=\alpha_{1} \cdot u+\alpha_{2} \cdot v+\alpha_{3}
$$

2.

$$
G(u, v)=\alpha_{1} \cdot u^{2}+\alpha_{2} \cdot v^{2}+\alpha_{3} \cdot u \cdot v+\alpha_{4} \cdot u+\alpha_{5} \cdot v+\alpha_{6}
$$

3.

$$
G(u, v)=\alpha_{1} \cdot u+\alpha_{2} \cdot v+\alpha_{3} \cdot u \cdot v+\alpha_{4}
$$

4.

$$
\begin{aligned}
G(u, v)= & \alpha_{1} \cdot u^{2}+\alpha_{2} \cdot v^{2}+\alpha_{3} \cdot u^{2} \cdot v+\alpha_{4} \cdot u \cdot v^{2}+ \\
& \alpha_{5} \cdot u \cdot v+\alpha_{6} \cdot u+\alpha_{7} \cdot v+\alpha_{8}
\end{aligned}
$$

5 .

$$
\begin{aligned}
G(u, v)= & \alpha_{1} \cdot u^{2} \cdot v^{2}+\alpha_{2} \cdot u^{2}+\alpha_{3} \cdot v^{2}+\alpha_{4} \cdot u^{2} \cdot v+ \\
& \alpha_{5} \cdot u \cdot v^{2}+\alpha_{6} \cdot u \cdot v+\alpha_{7} \cdot u+\alpha_{8} \cdot v+\alpha_{9}
\end{aligned}
$$

wobei $u$ und $v$ die jeweiligen Elementkoordinaten bezeichnen. Die Koeffizienten $\alpha_{i}$ werden aus der Interpolationsbedingung für die Elementknoten bestimmt.

Bei einer Interpolation ohne Bedingung der Konservativität werden die Knoten des Zielgitters in das Sendergitter projiziert. Sie erhalten dann jeweils den Wert, der sich aus ihrer Projektionslage und der entsprechenden Formel des Elementtyps ergibt. Bei der Interpolation unter Bedingung der Konservativität werden die Knoten des Sendergitters in das Zielgitter projiziert. Der Wert an den Senderknoten wird verteilt nach der Formel des Elementtyps. Ist der Wert an den Elementzentren angeordnet, kann konservativ, d.h. unter Bedingung der Konservativität, in dieser Weise nur von den Elementzentren auf Knoten geschickt werden. Bei der Interpolation ohne Bedingung der Konservativität kann so nur von Knoten auf Elementzentren gesendet werden. Alle anderen Varianten erfordern für die gleiche Art der Interpolation die Konstruktion dualer Gitter, welches derzeitig in MpCCI noch nicht unterstützt wird.

\subsection{Funktionen für User-Subroutines}

Benutzerdefinierte Interpolation über Matrizenspezifikation. Die Simulation verschiedener physikalischer Phänomene führt zu unterschiedlichen Anforderungen an die Interpolationsroutinen einer Kopplungsbibliothek. Zusätzlich zu den existierenden Standardinterpolationen, welche einen internen Teil von MpCCI darstellen, bietet MpCCI eine Schnittstelle für benutzerdefinierte Interpolationen. Dieses Interface ermöglicht dem Benutzer seine eigene Nachbarschaftssuche und Interpolation in MpCCI zu integrieren, so dass MpCCI 
basierend auf dieser speziellen Interpolationsvorschrift den Datentransfer realisiert.

Die benutzerdefinierte Interpolations-Schnittstelle besteht zu einem Teil aus speziellen „Query-Funktionen“. Diese Funktionen können benutzt werden, um alle Informationen über ein beliebiges, interessierendes Gitterpaar einer Kopplungsregion aus MpCCI einer speziellen Interpolationsroutine zur Verfügung zu stellen. Der Benutzer kann über diese Funktionen die Anzahl der an einem der Kopplungsgitter beteiligten Prozesse, d.h. die Prozesse auf denen Teile des Kopplungsgitters gespeichert sind und bearbeitet werden, sowie deren Identifikationsnummern abfragen. Danach kann der Benutzer mit diesen Funktionen die Anzahl der Partitionen, auf die Teile des Kopplungsgitters auf jedem der vorher ermittelten Prozesse aufgeteilt sind, und wiederum die Identifikationsnummern dieser Partitionen erfragen. Weiter erhält er die gesamten Informationen über Knoten und Elemente einer spezifizierten Partition, die in MpCCI verfügbar sind, durch spezielle „Query-Funktionen“. Der Benutzer kann dann auf diese aus MpCCI erhaltenen Gitter seine eigenen Interpolationsalgorithmen anwenden. Den zweiten Teil der Interpolationsschnittstelle bilden die Funktionen zur Definition der Interpolationsmatrizen. Der Benutzer muss zwei Matrizen für jedes Paar von Gitterpartitionen spezifizieren. Die Matrizen werden Restriction- und Injection-Matrix genannt. Die Restriction-Matrix wird auf den Vektor angewendet mit den Werten, die an den Knoten oder Elementen der zu der Matrix gehörigen Senderpartition angeordnet sind. Der resultierende Vektor wird zu der Empfängerpartition gesendet, die in der Definition der Restriction-Matrix spezifiziert ist. Es existieren also für jede Partition der Senderprozesse die gleiche Anzahl RestrictionMatrizen wie die Anzahl der Partitionen aller Empfängerprozesse. Auf dem Empfängerprozess werden die Injection-Matrizen, die zu der Empfängerpartition und zu jeder der Senderpartitionen gehören, auf jeden der entsprechenden, empfangenen Vektoren angewendet. Die Summe der resultierenden Vektoren ist der Vektor mit den Werten, die an den Knoten oder Elementen der Empfängerpartition angeordnet werden (siehe Abbildung 15). Hinsichtlich des zweiten Teils der Interpolationsschnittstelle ist für bestimmte Fälle eine Alternative implementiert worden. Diese alternative Implementierung bietet die Möglichkeit, die interpolierten Werte ohne Spezifikation der Interpolationsfunktion in Matrizenform zu berechnen. So sind mit dieser Schnittstelle auch Interpolationen, die nicht auf einer linearen Abbildung beruhen oder sich auf Grund des Verfahrens nicht in der Kopplungs-Definitionsphase als Matrix spezifizieren lassen, an MpCCI anzubinden und damit zu realisieren.

Die ersten Verfahren dieser Arbeit, in denen die Matrizen über ein direktes Verfahren invertiert werden, wurden über die erste oben spezifizierte Schnittstelle an MpCCI angebunden. Die Matrizen wurden in den speziellen Routinen berechnet und dann nach weiteren Modifikationen an MpCCI - wie oben beschrieben - spezifiziert. Die zweite, alternative Schnittstelle wurde für diese Arbeit nach der Implementation von Funktionen angewendet, die zur Berechnung der Matrix Ergebnisse der Simulationscodes benötigen, die erst 
Senderprozess

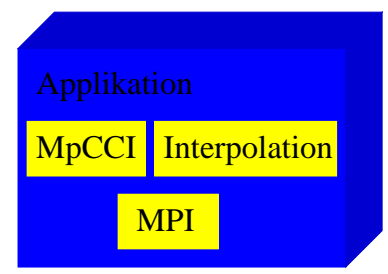

$\mathrm{R}_{1} \mathrm{~S}=\mathrm{V}_{1}$
Empfaengerprozess

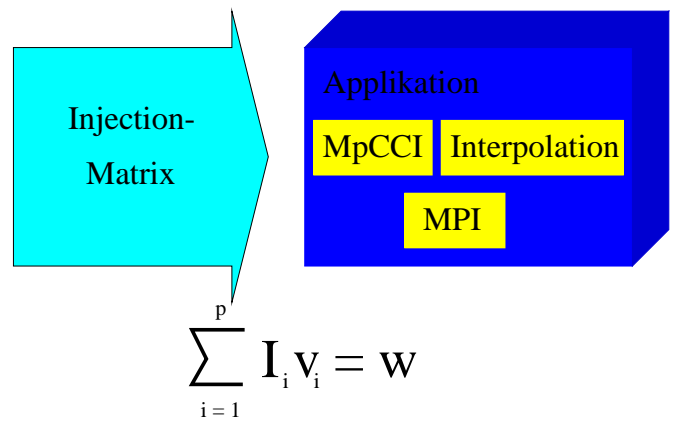

Abbildung 15. Für jedes Paar von Partitionen muss eine Restriction- und Injection-Matrix definiert werden. Die Abbildung stellt den Ablauf des auf diesen Matrizen basierenden Datentransfers zwischen einem Partitionspaar dar.

in der gekoppelten Berechnung zur Verfügung stehen, oder iterative Löser verwenden.

In anderen Projekten mit MpCCI wird die neue Schnittstelle unter anderem auch zur Interpolation über einen Neutralflächen-Ansatz unter Einsatz von NURBS-Formulierungen (Non Uniform Rational B-Splines) eingesetzt.

Für die iterativen Löser in dieser Arbeit wird die Schnittstelle aus zwei Gründen benötigt. Die implementierte Schnittstellenfunktion zur Definition der Interpolationsmatrizen muss in der Kopplungsdefinitions-Phase aufgerufen werden, in der die notwendige rechte Seite des Gleichungssystems generell noch nicht berechnet ist. Die rechte Seite des Gleichungssystems wird durch die zu interpolierenden Werte des Sendergitters gegeben. Diese Werte sind aber in der Kopplungsdefinitions-Phase noch nicht vorhanden, sondern im Allgemeinen erst in der darauffolgenden Phase, wenn die Werte jeweils bei einem CCI_Send an MpCCI spezifiziert werden. Der andere Grund ist, dass das Ergebnis des iterativen Lösers keine invertierte Matrix ist, sondern konkret der Lösungsvektor des Gleichungssystems. Dieser Lösungsvektor wird dann im Algorithmus der jeweils implementierten Methode zur Berechnung der Interpolationswerte auf dem Empfängergitter verwendet. Es wird also keine Matrix bestimmt, die zur Interpolation der im weiteren Verlauf der gekoppelten Simulation spezifizierten Kopplungswerte angewendet werden kann.

Benutzerdefinierte Interpolation ohne Matrizenspezifikation. Die zweite implementierte Schnittstelle besteht aus vier neuen Funktionen. Mit der ersten Funktion wird durch einen Funktionszeiger die Funktion zur Vorbereitung der Interpolation in der Kopplungsdefinitions-Phase spezifiziert. In dieser Funktion können dann über die Schnittstellenfunktion, die auch zur Definition der Matrizen in der ersten Implementierung verwendet wird, die Funktionszeiger auf die beiden anderen neuen Funktionen übergeben werden. Eine der beiden Funktionen wird dann - wie schon aus den vorherigen Ausführungen folgt bei dem Einsatz von CCI_Send auf dem Senderprozess und die andere in 
der Funktion $C C I_{-} R e c v$ auf dem Empfängerprozess aufgerufen und zwar für jedes Paar von möglichen Sender- und Empfängerpartitionen.

Wie bei den Restriction- und Injection-Matrizen wird bei der Funktion das Paar von Sender- und Empfängerpartition spezifiziert, so dass auch hier für jede Partition auf einem Senderprozess die gleiche Anzahl von Funktionen bei einem CCI_Send aufgerufen wird, wie die Anzahl der Partitionen aller Empfängerprozesse. Ebenso wird für jede Partition auf einem Empfängerprozess die gleiche Anzahl von Funktionen bei einem CCI_Recv aufgerufen, wie Partitionen aller Senderprozesse existieren.

Bei der Implementation dieser Funktionen muss also wieder genau darauf geachtet werden, dass ein spezielles Paar von Empfänger- und Senderpartition behandelt wird.

Wie bereits beschrieben, wird für ein spezielles Paar von Sender- und Empfängerpartition eine Funktion von CCI_Send und eine andere Funktion von $C C I_{-} R e c v$ aufgerufen. In dieser Arbeit wird immer eine der Funktionen zur Koordinatensystem-Transformation genutzt und in der jeweils anderen werden die kompletten Berechnungen zur Interpolation implementiert. Die rechte Seite des Gleichungssystems wird der Funktion über einen ihrer Parameter durch einen Zeiger übergeben. Der resultierende Lösungsvektor wird dann zur Interpolation der Werte weiter verwendet. Je nachdem welche Funktion die Interpolationsberechnungen startet, werden an CCI_Send oder $C C I_{-} R e c v$ die interpolierten Werte gegeben. Von welcher der Funktionen die Koordinatensystem-Transformation oder Interpolationsberechnungen aufgerufen werden, wird durch die Art der Interpolation bestimmt.

Die Möglichkeit einer benutzerdefinierten Nachbarschaftssuche und Interpolation bietet einen einfachen Weg, um multidisziplinäre Simulationen mit speziellen Anforderungen zu realisieren.

\section{Die Interpolationsbibliothek AKITA}

\subsection{Problembeschreibung}

Die Interpolationsschnittstelle ist besonders von Interesse, wenn eine problemspezifische Interpolation gefordert ist; dafür ist gerade die Aeroelastik ein typisches Beispiel. Die derzeitig in MpCCI implementierten Standardinterpolationen sind für die Aeroelastik nicht ausreichend.

Bei der losen Kopplung in der Aeroelastik ist die Wechselwirkung auf den Austausch von Randbedingungen zwischen einem Strömungscode und einem Strukturcode beschränkt. Die aerodynamischen Drücke, berechnet auf finiten Volumen, Volumen-Elementen oder Panels im Strömungscode, werden über die entsprechenden Flächen zu Kräften aufintegriert. Die Kräfte werden dann auf die Strukturknoten interpoliert, so dass die passenden Lasten auf die Struktur wirken. Ebenso werden die im Strukturcode ermittelten Verschiebungen und Rotationen an den Strukturknoten als Verschiebungen auf 
die Strömungsknoten interpoliert, um im Strömungscode die Berechnung für die neue Geometrie durchzuführen.

In den meisten Fällen überzieht das Strukturgitter nur den Kern des Flügels. Dadurch ist eine orthogonale Projektion von allen Strömungsknoten in eines der Strukturelemente nicht möglich und auch teilweise für die Knoten, die sich in ein Strukturelement projizieren lassen, nicht sinnvoll. Die Standardinterpolationen ${ }^{1}$ setzen aber auf diesen Projektionen auf. Ein spezielles, vom Benutzer konfigurierbares Kriterium (siehe Abschnitt 1.5) regelt für die nicht in ein Strukturelement projizierbaren Knoten, ob sie in die Kopplung einbezogen werden oder nicht. Dieses Kriterium kann so gesetzt werden, dass alle Knoten noch einem Element zugeordnet werden und damit in die Kopplung eingehen. Wird eine Standardinterpolation in der Weise benutzt, kann aber gezeigt werden, dass, beispielsweise bei Verschiebungen durch Starrkörperbewegungen auf der Strukturseite, mit den auf die Strömungsknoten interpolierten Verschiebungen die Form der Strömungsoberfläche nicht bestehen bleibt. Starrkörperbewegungen werden mit den Standardinterpolationen also nicht erhalten. Schon aus diesem Grund sind die Standardinterpolationen für diese Art der Anwendungen nicht ausreichend. Allgemein sind die Standardinterpolationen nur so lange sinnvoll, wie vernünftige Projektionen der Knoten in die gegenüberliegenden Elemente existieren, abgesehen von weiteren Bedingungen, die an die Interpolationen gestellt werden. Darüber hinaus ist für die Interpolationsfunktionen der Interpolation ohne Bedingung der Konservativität an den Elementkanten nicht notwendigerweise Differenzierbarkeit zu erreichen. Bei den konservativ durchgeführten Interpolationen können keine Aussagen über die Interpolationsfunktion getroffen werden, da hier keine Interpolation nach einer Funktion erfolgt, denn die Verteilungsformel ist abhängig von dem Auswertungsgitter. Die Interpolationen mit und ohne Bedingung der Konservativität basieren auf der Elementstruktur, ohne die sie nicht durchführbar sind.

\section{$2.2 \quad$ Existierende Software}

Am Institut für Aeroelastik des DLR ist von Beckert ein spezielles Kopplungstool mit dem Namen CAESAR entwickelt worden. Es umfasst mehrere Prozeduren, die die zeitliche Kopplung regeln, sowie eine Auswahl von räumlichen Kopplungsverfahren. Dies sind Algorithmen, die speziell für die Kopplungsprobleme auf dem Gebiet der Aeroelastik [3], [2] angepasst wurden. Die Verfahren sind natürlich auch darüber hinaus auf andere multidisziplinäre Probleme anwendbar. Wie in der Beschreibung zu den Interpolationen mit radialen Basisfunktionen gezeigt wurde, sind diese nicht nur zwischen Flächen im dreidimensionalen Raum anwendbar, sondern auch für Kopplungen zwischen Volumen im dreidimensionalen Raum oder auch zwischen Gebieten

\footnotetext{
${ }^{1}$ Mit Ausnahme der Nearest-Neighbor-Interpolation, welche aber schon durch die Ungenauigkeit und mangelnde Glattheit als Interpolation bei den Verschiebungen nicht anzuwenden ist.
} 
anderer, auch unterschiedlicher Dimensionen, z.B. für Kopplungen zwischen Linien und Flächen im dreidimensionalen Raum zu verwenden. Wie bereits beschrieben sind Interpolationen mit radialen Basisfunktionen generell im n-dimensionalen Raum möglich.

Der Teil von CAESAR, der aus den Interpolationsalgorithmen für den Raum besteht, ist von Armin Beckert herausgetrennt und mit KopMat bezeichnet worden. Die darin implementierten Routinen bestehen aus einem Algorithmus, der auf der Methode der finiten Interpolationselemente basiert, und sechs multivariaten Interpolationen, die radiale Basisfunktionen verwenden. Die Interpolationen mit radialen Basisfunktionen sind von Armin Beckert zusätzlich in einer $\mathrm{C}++$ Bibliothek mit Namen ABSCL implementiert worden.

\subsection{Datenumwandlung und Parallelisierung}

Anforderungen. Die Interpolationsmodule KopMat und ABSCL wurden über das oben beschriebene Interpolations-Interface mit MpCCI verbunden. Auf Grund der parallelen Situation und der verschiedenen Datenstrukturen musste eine Art Transformationsmodul geschrieben werden, das zum einen die Gitterinformationen für KopMat bzw. ABSCL aufbereitet und zum anderen die Matrix, die das Resultat von KopMat/ABSCL darstellt, in die Injectionund Restriction-Matrizen der vorhandenen Partitionspaare verteilt. Bei der parallelen Situation muss besonders beachtet werden, dass Knoten auf verschiedenen Partitionen mit gleichen Koordinaten existieren können. Diese Knoten werden später auch als identische Knoten bezeichnet.

Bei der Aufstellung der Injection- und Restriction-Matrizen bzw. auch bei der Implementation der Funktionen, die in CCI_Send und CCI_Recv eingebunden werden, müssen nun hinsichtlich der Empfängerseite alle Knoten berücksichtigt werden, das heißt, auch die mehrfach auftretenden Knoten müssen hier in gleicher Weise bedacht werden. Es muss vermieden werden, dass Knoten, die auf den gleichen Koordinaten liegen, aber unterschiedlichen Partitionen angehören, unterschiedliche Werte erhalten. Auf der Senderseite dagegen darf nur einer der mehrfach auftretenden Knoten berücksichtigt werden. Hier muss erreicht werden, dass die versendeten Werte nicht mehrfach eingehen, sonst würde z.B. ein Wert, der an einem Knoten angeordnet ist, der mit den selben Koordinaten in drei Partitionen auftritt, dreimal in der Berechnung berücksichtigt. So müssen die Knotenwerte beim Senden und Empfangen unterschiedlich behandelt werden.

Bei allen in KopMat und ABSCL vorhandenen Interpolationsalgorithmen wird die Matrix zur Interpolation der Verformungen von den Strukturknoten auf die Strömungsknoten berechnet. Die Transformation der Kräfte von Strömungsknoten auf Strukturknoten wendete bisher die transponierte der berechneten Kopplungsmatrix an. Darüber werden die Bedingungen der Konservativität und der Äquivalenz der Arbeit realisiert, wie in Kapitel II Abschnitt 2.1 und 2.2 beschrieben wird (siehe auch [2]). Im parallelen Fall 
ist es jedoch nicht möglich, die transponierte Matrix ohne Veränderungen zu verwenden. Der zuvor genannte Punkt geht hier ein: Unabhängig von dem Verfahren mit dem die Matrix aufgestellt worden ist, bei der Umkehrrichtung müssen die Knotenwerte immer anders behandelt werden. Es reicht hier nicht - im Gegensatz zum seriellen Fall - die transponierte Matrix aufzustellen.

Realisierung. Mit dem Transformationsmodul wird auch eine Parallelisierung, so weit sie außerhalb von KopMat/ABSCL machbar ist, realisiert. In den folgenden Abschnitten wird die gesamte Datenumwandlung von MpCCI zu KopMat/ABSCL und umgekehrt mit der Parallelisierung für die Interpolationen auf Grundlage radialer Basisfunktionen vereinfacht beschrieben. Danach folgt die Beschreibung für die Interpolationen auf Basis der Methode der finiten Interpolationselemente, soweit sie von der ersten abweicht. Im letzten Teil wird die Verwendung des Transformationsmoduls mit der neuen, alternativen Interpolations-Schnittstelle von MpCCI erläutert.

Die Routinen des Transformationsmoduls werden ebenso wie die MpCCIRoutinen auf jedem Prozess der gekoppelten Anwendung aufgerufen (siehe Abbildung 18). Im nachfolgenden Text wird das Verfahren aus der Sicht eines beliebigen Prozesses der Anwendung erklärt. Die Bezeichnung „lokal“ wird verwendet, wenn es sich um den Code handelt, zu dem der eigene Prozess gehört; die Bezeichnung „entfernt“ wird dementsprechend für Teile des gegenüberliegenden Codes der gekoppelten Anwendung benutzt. Die Beschreibung erfolgt für die Interpolation zwischen genau einem Gitterpaar. Bei mehreren Gitterpaaren werden die Routinen insgesamt mehrfach durchlaufen.

Zunächst werden auf jedem Prozess der Strömungs- und der Strukturseite die Anzahl der am lokalen Kopplungsgitter beteiligten Partitionen auf dem jeweiligen Prozess und deren Identifikationsnummern erfragt. Dann wird ein Zeiger auf die Knotenkoordinaten jeder Partition aus MpCCI geholt. Im nächsten Teil wird die Anzahl der am entfernten Kopplungsgitter beteiligten Prozesse und deren Identifikationsnummern erfragt. Für jeden Prozess werden dann die jeweilige Anzahl der am Kopplungsgitter beteiligten Partitionen und deren Identifikationsnummern von MpCCI erhalten. Dann werden auch für das entfernte Gitter Zeiger auf die Knotenkoordinaten jeder Partition jedes Prozesses aus MpCCI geholt. Die Gitterpartitionen des eigenen und des gegenüberliegenden Codes, die jeweils auf einem Prozess bearbeitet werden, sind in Abbildung 16 zum besseren Verständnis skizziert.

Die Knotenkoordinaten des entfernten Gitters werden auf Knoten durchsucht, die durch die parallele Situation in mehreren Partitionen auftauchen. Die Suche wird über eine externe, frei zugängliche Bibliothek "ANN" [9] realisiert, die mit Datenstrukturen auf Grundlage von „kd-trees" und „boxdecomposition trees" arbeitet. Es werden mehrere Indexlisten geführt. Die erste wird an die Interpolationsbibliothek weitergegeben und ist einfach eine durchgehende Nummerierung der Knoten. Durch die zweite Liste wird die Abbildung der parallelen Identifikationsnummern auf die seriellen Identifikationsnummern erhalten. Die dritte stellt die Abbildung der seriellen Iden- 


\section{CSM}

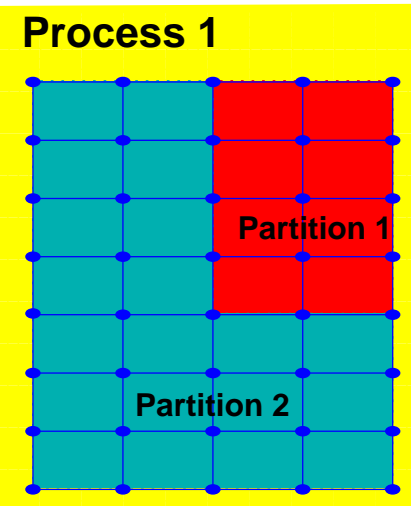

Process 2

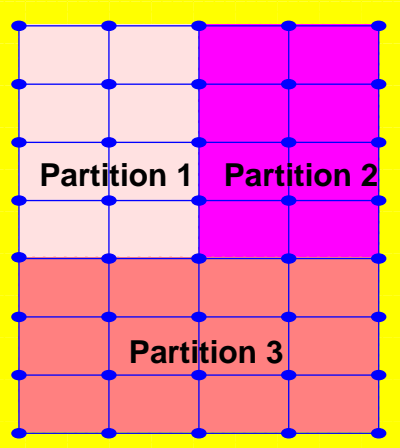

CFD

\section{Process 1}

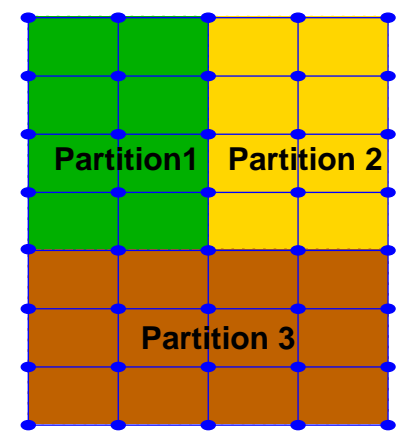

\section{Process 2}

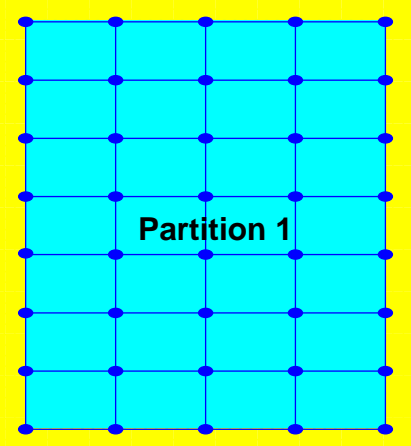

Process 3

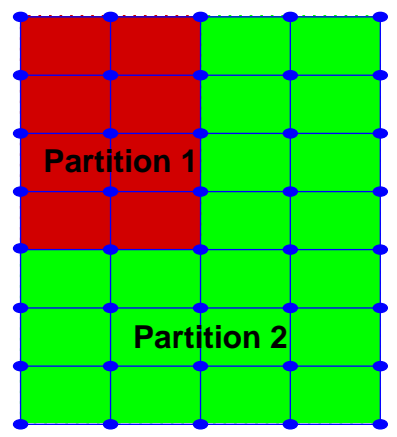

Abbildung 16. Die Skizze zeigt einen Strukturcode mit 2 Prozessen und einen Strömungscode mit 3 Prozessen mit ihren jeweiligen Gitterpartitionen. Die gelb unterlegten Partitionen sollen beispielhaft die auf Prozess 2 benötigten Gitterpartitionen des Strömungscodes zur Berechnung der Interpolationsmatrizen zeigen.

tifikationsnummern auf je eine der parallelen Identifikationsnummern dar. Parallele Identifikationsnummer heißt, dass jedem Knoten, ob mehrfach auftretend oder nicht, eine fortlaufende Nummer über alle Partitionen und Prozesse zugeordnet wird; serielle Identifikationsnummer bedeutet, dass allen voneinander verschiedenen Knoten je eine Nummer zugeordnet wird.

Die Interpolationsalgorithmen unter Verwendung der radialen Basisfunktionen werden dann auf den Prozessen der Strömungsseite mit allen Knoten aller Partitionen aller Prozesse der Strukturseite und allen Knoten aller 
Partitionen des jeweiligen Strömungscode-Prozesses gestartet. Jeder Prozess erhält nach der Berechnung eine Matrix, die relevant ist für die Partitionen des Strömungscode-Prozesses, auf dem sie berechnet wurde, und für alle Partitionen aller Strukturcode-Prozesse. Für jeden der Strukturcode-Prozesse ist aber nur ein Teil der Matrix jedes Strömungscode-Prozesses interessant. So werden auf den Strömungscode-Prozessen die Matrizen in Teile zerlegt, die jeweils zu einem Strukturcode-Prozess passen. Die Zerlegung erfolgt auf Basis der Indexlisten. Dann wird von jedem Strömungscode-Prozess der jeweils passende Teil der Matrix zu jedem der Strukturcode-Prozesse auf MPI-Basis geschickt. Auf jedem der Strukturcode-Prozesse werden die Teile der Matrizen aller Strömungscode-Prozesse empfangen und wieder zu einer Matrix zusammengesetzt. Jetzt sind sowohl auf allen Strömungscode-Prozessen als auch auf den Strukturcode-Prozessen alle nötigen Matrizen vorhanden.

Die Matrizen liegen aber nicht in einer Form vor, in der die Koeffizienten aller Zeilen hintereinander abgespeichert sind, sondern es gibt zwei Indexlisten, in denen die Zeile und Spalte angegeben ist, und eine Liste, in der die zugehörigen Koeffizienten stehen (Sparse-Matrix-Form). MpCCI benötigt die Matrizen in einer komplett anderen Form: An die Kopplungsbibliothek muss die Anzahl der von Null verschiedenen Einträge in jeder Zeile spezifiziert werden. So muss hier die Matrixformulierung noch komplett umgesetzt werden. Außerdem hat jeder Strömungscode- und Strukturcode-Prozess die Matrix für alle Partitionen, die auf dem jeweiligen Prozess liegen, und alle Partitionen aller entfernten Prozesse. Da die Matrizen aber, wie schon mehrfach erwähnt, für jedes Partitionspaar benötigt werden, muss der Algorithmus auch hier aufspalten. Auf der Strukturseite müssen jetzt, anhand der vorher aufgestellten Indexlisten, die Koeffizienten 0 gesetzt werden, die durch identische Knoten auf der Strömungsseite aufgetreten sind. Der gesamte beschriebene Transformationsprozess ist in Abbildung 17 vereinfacht dargestellt.

Bei der Interpolation auf Grundlage der finiten Interpolationselemente [3] wird ähnlich vorgegangen. Ein grundsätzlicher Unterschied ist, dass hier die Elemente für die Interpolation benötigt werden. So werden hier auch die Elementtypen und Elemente aus MpCCI mit ihren Knotenlisten aus MpCCI abgegriffen. In den Knotenlisten der Elemente müssen die entsprechenden Identifikationsnummern nach den oben erklärten Indexlisten umgesetzt werden. Dann werden neu aufgebaute Arrays an KopMat gegeben, da auch bei den Elementlisten Unterschiede zwischen den Datenstrukturen bestehen. Bei den erhaltenen Matrizen muss beachtet werden, dass für ein voneinander abhängiges Knotenpaar nicht nur ein Koeffizient abgespeichert werden muss, sondern eine $6 \times 6$-Matrix, welches den Algorithmus stark verändert.

Da die Knotenkoordinaten an MpCCI in unterschiedlichen Koordinatensystemen spezifiziert werden können, muss das Transformationsmodul auch Koordinatensystem-Transformationen durchführen. Die Lage aller Koordinatensysteme wird vom Benutzer gegenüber einem globalen Koordinatensystem im MpCCI-Inputfile angegeben. MpCCI berechnet die Koordinaten- 

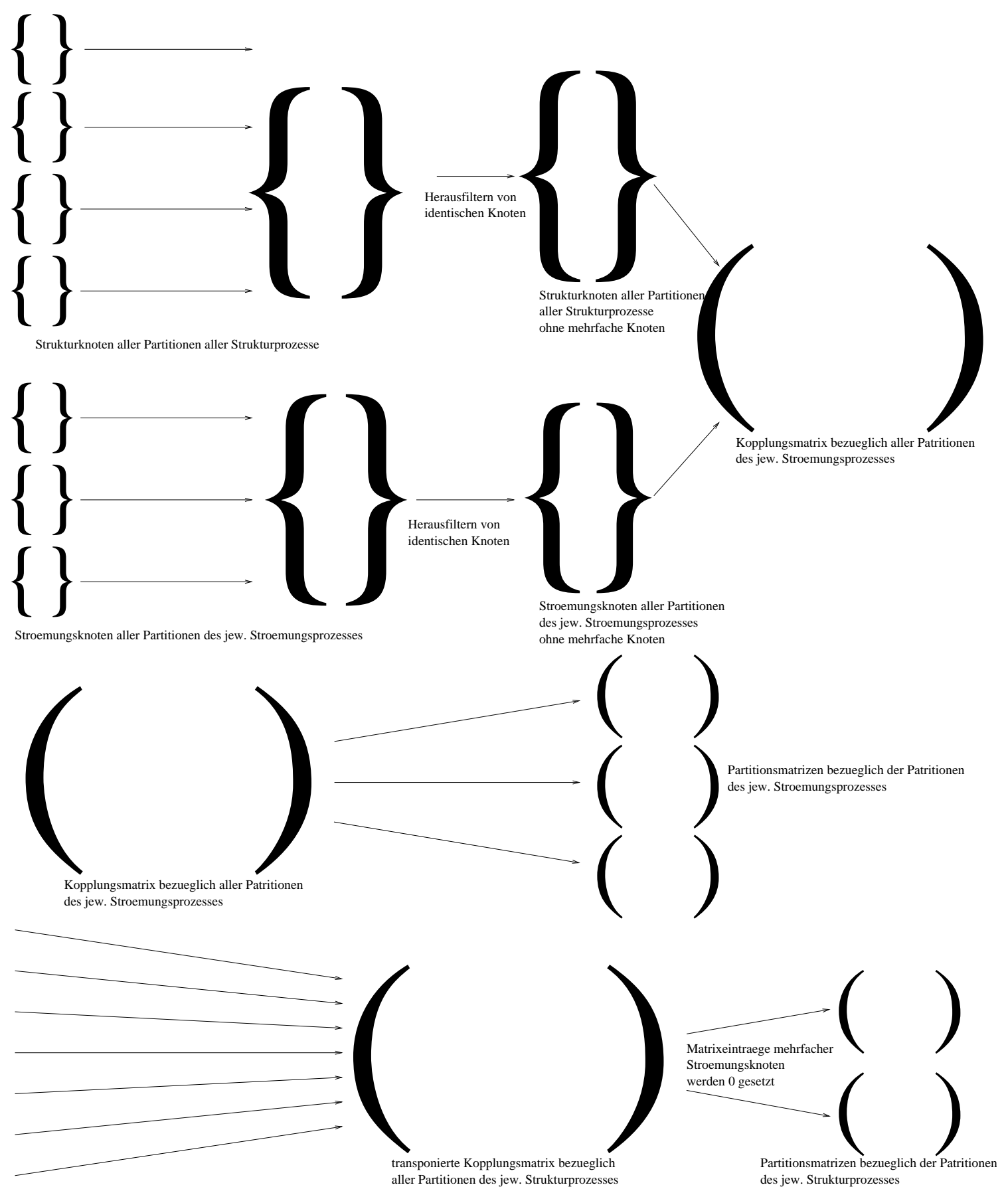

Teile der transponierten Kopplungsmatrix die die Patritionen des jew. Strukturprozesses betreffen werden mit MPI von den

Stoemungsprozessen zu dem jew. Strukturprozess gesendet

Abbildung 17. Der schematisch dargestellte Transformationsprozess der Eingabedaten von MpCCI zur Interpolationsbibliothek und der berechneten Daten von der Interpolationsbibliothek zu MpCCI zurück.

system-Transformationsmatrizen aus dieser Spezifikation. Die Koordinaten werden immer in das Koordinatensystem transformiert, welches in dem Code Grundlage ist, in dem die Berechnung der Interpolationsmatrix gestartet wird. Bei dieser Koordinatensystem-Transformation müssen natürlich Translation, Rotation und Skalierung der Achsen der Koordinatensysteme zu- 
einander berücksichtigt werden. Beim späteren Austausch der Kräfte und Verformungen müssen die unterschiedlichen Koordinatensysteme ebenfalls Berücksichtigung finden. Dies geschieht über die Restriction-Matrizen, die die Koordinatensystem-Transformationsmatrizen für die jeweiligen Partitionspaare darstellen. Hier darf aber nur die Rotation und Skalierung der Achsen der Koordinatensysteme zueinander eingehen.

Wird für die Interpolationen aus den im Abschnitt 1.6 erläuterten Gründen die neue, alternative Interpolations-Schnittstelle zu MpCCI angewendet, so wird für die Funktion, die von MpCCI während der KopplungsdefinitionsPhase aufgerufen wird, ebenso vorgegangen, wie oben beschrieben: Als erstes werden die gesamten Gitterinformationen über die „Query-Funktionen“ für jeden Prozess der gekoppelten Simulation von MpCCI geholt und wie oben für die Interpolationsbibliothek aufbereitet. Auch bei Verwendung dieser Schnittstelle müssen die folgenden drei Listen angelegt werden: Die durchgehende Nummerierung der voneinander verschiedenen Knoten, die Abbildung der parallelen Identifikationsnummer auf die serielle Identifikationsnummer und die Abbildung der seriellen Identifikationsnummer auf die parallele Identifikationsnummer. Im Gegensatz zum Vorgehen bei Verwendung der ersten Interpolations-Schnittstelle von MpCCI werden dann aber in dieser Funktion nicht mehr die kompletten Matrizen aufgestellt und invertiert, sondern es werden nur Vorbereitungen durchgeführt oder Teile der Matrizen berechnet, soweit wie mit dem jeweilig verwendeten Algorithmus an diesem Punkt des Verfahrens möglich. Über die Schnittstellenfunktionen, die auch zur Definition der Restriction- und Injection-Matrizen verwendet werden, werden Funktionszeiger auf die Funktionen übergeben, die bei CCI_Send und CCI_Recv in der gekoppelten Simulation aufgerufen werden sollen. Der Funktion, die von $C C I \_S e n d$ aufgerufen wird, werden die Kopplungswerte, wie z.B. Deformationen oder Kräfte des Senderprozesses der spezifizierten Partition übergeben. In der Funktion werden die Werte bestimmt, die an die angegebene Empfängerpartition eines ebenfalls spezifizierten Prozesses übertragen werden soll. Diese Werte werden in CCI_Send über MPI an den Empfängerprozess gesendet und im CCI_Recv an die dort aufgerufene Funktion mit spezifiziertem Senderprozess, Senderpartition und Empfängerpartition übergeben. Diese Funktion berechnet schließlich die Werte, die an CCI_Recv zurückgegeben werden und später auf die Knoten der Empfängerpartition gegeben werden. In beiden Funktionen müssen Routinen des Transformationsmoduls aufgerufen werden, um über die in der Kopplungsdefinitions-Phase erstellten Listen die für die parallele Situation gegebenen Werte in eine serielle Situation zu transformieren und umgekehrt. Darüber hinaus müssen auf dem Senderprozess, z.B. um die Kräfte über alle Knoten aufsummieren zu können, über MPI aus allen Partitionen jedes Senderprozesses die Werte zusammengestellt werden (siehe Abbildung 18). Auf welcher Seite die Interpolationen durchgeführt werden ist abhängig vom gewählten Algorithmus; die Koordinatensystem-Transformationen werden immer auf der jeweils anderen Seite berechnet. 


\section{Prozess der gekoppelten Anwendung}

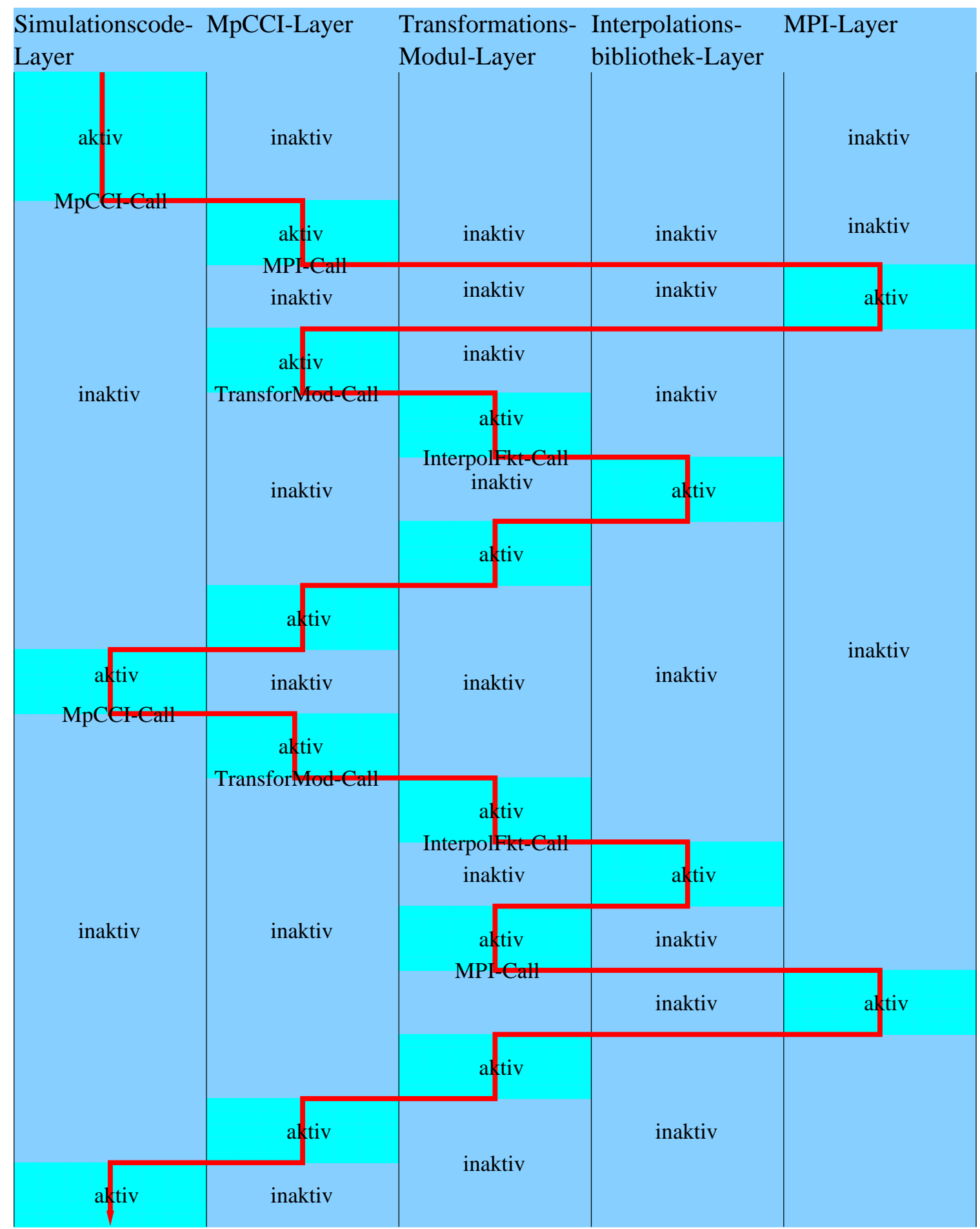

Abbildung 18. Dieses Beispiel zeigt die Abfolge der aktiv werdenden Layer bei dem Aufruf der Interpolation aus MpCCI im CCI_Close_Setup (oben) und im CCI_Recv (unten).

Das Transformationsmodul ist unter folgenden Zielen entwickelt worden:

1. Es sollten möglichst auf keinem Prozess alle Gitterknoten vorhanden sein. 
2. Bei der Parallelisierung sollten so wenig wie möglich gleiche Berechnungsschritte auf gleiche Daten angewendet werden.

3. Die notwendige Kommunikation auf MPI-Basis sollte gering gehalten werden.

4. Die Prozesse sollten nur aufeinander warten müssen, wenn es unvermeidbar ist.

Das Transformationsmodul ist in $\mathrm{C}++$ geschrieben und wird als Bibliothek ebenso wie die Interpolationsbibliotheken und MpCCI an die Anwendungscodes gelinkt.

Der Interpolationsbibliothek, die aus KopMat/ABSCL entstanden ist und alle neu entwickelten und verbesserten Algorithmen sowie das Transformationsmodul enthält, wurde der Projektname „AKITA“ gegeben. AKITA ist in $\mathrm{C}++$ geschrieben und verwendet für den internen Datenaustausch MPI. Über Konfigurationsfiles kann AKITA so übersetzt und gelinkt werden, dass AKITA auch ohne MpCCI über eine entsprechende Schnittstelle als Interpolationsbibliothek eingesetzt werden kann.

Der Algorithmus mit den entsprechenden Features kann über das MpCCI Input-File eingestellt werden. Im MpCCI Inputfile können auch die radiale Basisfunktion und die Anzahl der polynomialen Terme eingestellt werden. In der Tabelle 3 sind der Klassenname und der implementierte Algorithmus für die beiden Transformationsrichtungen aufgelistet.

Die in den Algorithmus eingehenden Parameter, wie Mindestanzahl der Stützstellen im Trägerradius, Vergrößerungsfaktor für den Trägerradius, Wahl der radialen Basisfunktionen, Anzahl der polynomialen Terme oder bei der "Partition of Unity“ Methode die Anzahl der Stützstellen in einer Partition, Größe der überlappenden Bereiche, Sortier-Reihenfolge der Koordinatenrichtungen, Partitionsaufteilung in den verschiedenen Koordinatenrichtungen können über das User Interface von AKITA spezifiziert werden.

In der Abbildung 19 ist die Vererbung der verschiedenen Klassen gezeigt und welche der Klassen mit Objekten hier ebenfalls dargestellter Klassen arbeiten. 


\begin{tabular}{|c|c|c|}
\hline Klassenname & \begin{tabular}{|l|} 
Algorithmus \\
Code1 auf Code2
\end{tabular} & $\begin{array}{l}\text { Algorithmus } \\
\text { Code2 auf Code1 }\end{array}$ \\
\hline AKIpolRBFGlobal & Global, Gauss & \\
\hline AKIpolRBFGlobal & Global, Gauss & $\begin{array}{l}\text { Global, } \\
\text { transponierte Matrix }\end{array}$ \\
\hline AKIpolRBFGlobalFSI & Global, LR & $\begin{array}{l}\text { Global, LR, Arbeitserhaltung, } \\
\text { Konservativität }\end{array}$ \\
\hline AKIpolRBFGlobalEoW & Global, LR & $\begin{array}{l}\text { Global, LR, } \\
\text { Arbeitserhaltung }\end{array}$ \\
\hline AKIpolRBFGlobalCons & \begin{tabular}{||l} 
Global, Gauss \\
Konservativität
\end{tabular} & \\
\hline AKIpolRBFGlobalConsEquiv & \begin{tabular}{|l} 
Global, Gauss \\
Arbeitserhaltung, \\
Konservativität
\end{tabular} & \\
\hline AKIpolRBFGlobalRotation & $\begin{array}{l}\text { Global, LR, } \\
\text { Rotationen }\end{array}$ & \\
\hline AKIpolRBFGlobalBiCGPrep & $\begin{array}{l}\text { Global, BiCG, } \\
\text { prepared }\end{array}$ & \\
\hline AKIpolRBFGlobalBiCGHalfPrep & $\begin{array}{l}\text { Global, BiCG, } \\
\text { half prepared }\end{array}$ & \\
\hline AKIpolRBFGlobalBiCGOffHand & $\begin{array}{l}\text { Global, BiCG, } \\
\text { off hand }\end{array}$ & \\
\hline AKIpolRBFGlobalAMG & Global, AMG & \\
\hline AKIpolRBFPoU & PoU, Gauss & \\
\hline AKIpolRBFPoU & PoU, Gauss & $\begin{array}{l}\text { PoU, } \\
\text { transponierte Matrix }\end{array}$ \\
\hline AKIpolRBFPoUFSI & PoU, LR & $\begin{array}{l}\text { PoU, LR, Arbeiterhaltung, } \\
\text { Konservativität }\end{array}$ \\
\hline AKIpolRBFPoUEoW & |PoU, LR & $\begin{array}{l}\text { PoU, LR, } \\
\text { Arbeitserhaltung }\end{array}$ \\
\hline AKIpolRBFPoUCons & \begin{tabular}{||l} 
PoU, Gauß, \\
Konservativität
\end{tabular} & \\
\hline AKIpolRBFPoUConsEquiv & $\begin{array}{l}\text { PoU, Gauß, } \\
\text { Arbeitserhaltung, } \\
\text { Konservativität }\end{array}$ & \\
\hline AKIpolRBFPoURotation & $\begin{array}{l}\text { PoU, LR, } \\
\text { Rotationen }\end{array}$ & \\
\hline AKIpolRBFGreedy & |Greedy, LR & \\
\hline AKIpolRBFGlobalApproximation & $\begin{array}{l}\text { Global, LR, } \\
\text { Approximation }\end{array}$ & \\
\hline AKIpolRBFPoUApproximation & $\begin{array}{l}\text { PoU, LR, } \\
\text { Approximation }\end{array}$ & \\
\hline
\end{tabular}

Tabelle 3. In der Tabelle sind die wichtigsten Klassen der Interpolationsbibliothek mit ihren implementierten Algorithmen aufgeführt. 


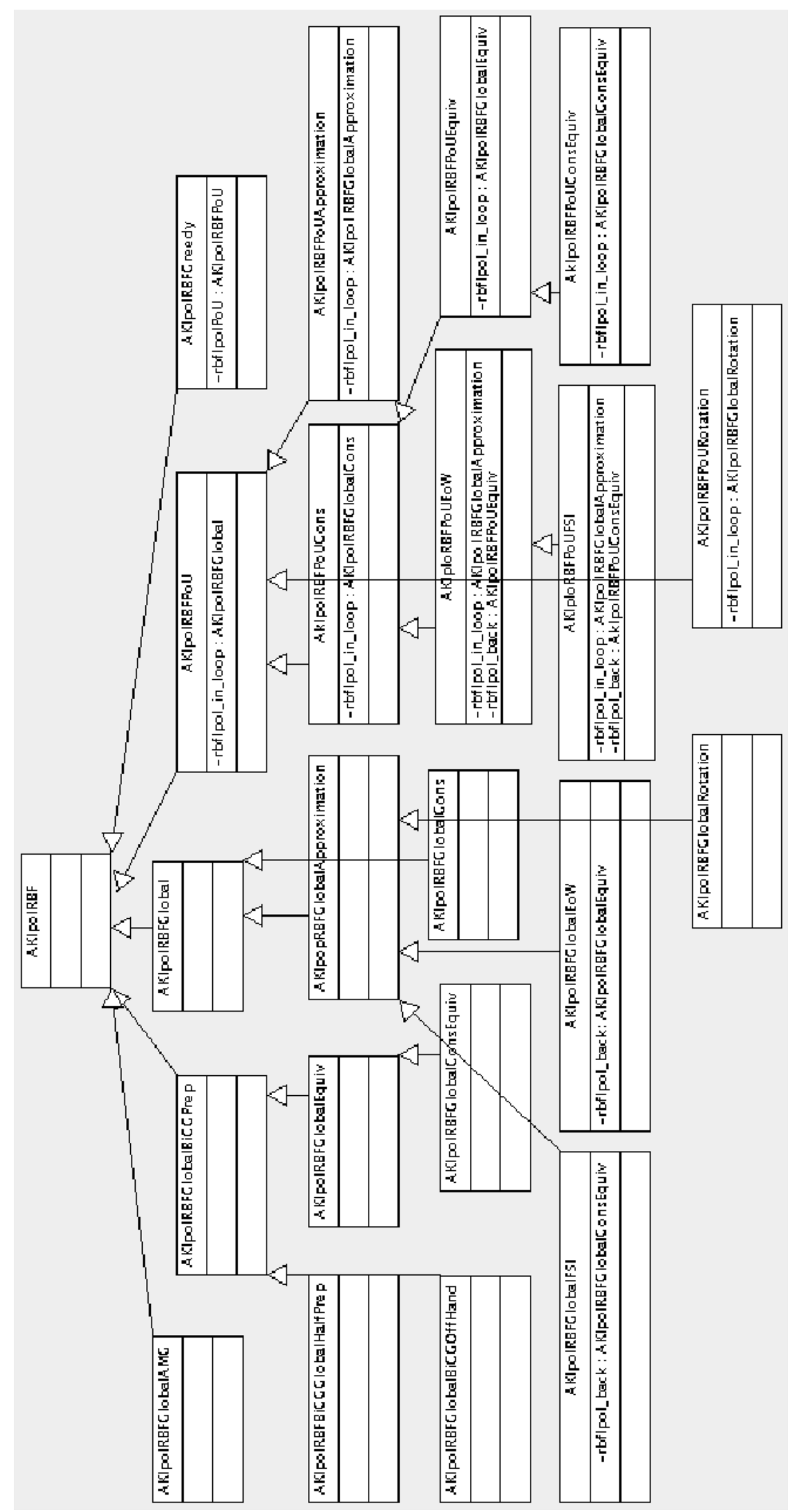

Abbildung 19. Die Abbildung zeigt eine Übersicht über die Vererbung der in AKITA implementierten Klassen und Objekte welcher der dargestellten Klassen in anderen Klassen verwendet werden. 


\section{Testbeispiele und Anwendungen in der multidisziplinären Simulation}

In den folgenden Abschnitten werden Anwendungsbeispiele für die verschiedenen Algorithmen der Interpolationsbibliothek demonstriert. Dies geschieht zunächst einzeln sowohl an durch analytische Funktionen generierten Werten als auch an bereits in einer Kopplung mit entsprechenden Simulationscodes berechneten Werten. Letztere sind auf den komplexen Geometrien von Flugzeug-Halbmodellen bzw. Flugzeug-Flügeln angeordnet. Es werden aber auch aus anderen Bereichen praxisnahe Beispiele für die Kopplung gezeigt.

Eine komplette gekoppelte Berechnung aus der Aeroelastik wird im Abschnitt 7 am Ende dieses Kapitels mit speziell zusammengestellten Interpolationsbedingungen und Verfahren präsentiert.

In den hier gezeigten aeroelastischen Beispielen werden immer die Deformationen von den Struktur- auf die Strömungsknoten zur Strömungsrechnung übertragen. Die Kräfte dagegen sind auf den Strömungsknoten gegeben und werden für die Strukturberechnung auf den Strukturknoten benötigt. So sind für die Interpolationen der Deformationen die Strukturknoten die Stützstellen und die Strömungsknoten die Auswertungsstellen, für die Interpolationen der Kräfte ist es genau umgekehrt. In den Abbildungen wird die Deformation immer farblich nach dem Betrag des Deformationsvektors dargestellt und die Knoten werden entsprechend des Deformationsvektors verschoben. Die Kraftvektoren werden durch an den Knoten angeordnete Pfeile und farblich nach dem Betrag des Kraftvektors visualisiert.

Bei allen Testfällen in den nächsten Abschnitten werden alle relevanten Daten in zwei Tabellen zusammengefasst. Die eine Tabelle enthält dabei die die Geometrie betreffende Information und findet sich im jeweiligen Abschnitt. Die Details über die verwendeten Interpolationen sind dagegen kompakt in den Tabellen im Anhang A zusammengefasst. Da diese Tabellen für alle Abschnitte den gleichen Aufbau haben, werden hier einige allgemeine Erklärungen zu den Tabellen gegeben.

Die erste und zweite Spalte der Tabellen über die Geometrie enthalten die Anzahl der für die Kopplung relevanten Knoten der beteiligten Codes. Aus der dritten Spalte ist ersichtlich, welche Knoten welches Codes in der Anwendung als Stützstellen dienen. Da bei der Präsentation einer aeroelastischen Anwendung mit der Transformation der Kräfte über Zusatzbedingungen in der Interpolation, die Stützstellen wie oben beschrieben, einmal die Strukturknoten und einmal die Strömungsknoten sind, enthält die Tabelle bei diesen Beispielen zwei Zeilen. Die erste Zeile ist für die Interpolation der Verschiebungen relevant, die zweite Zeile für die der Kräfte. In der vorletzten Spalte wird die Fülldichte $h_{X, \Omega}$ der Stützstellen, d.h. in den meisten Fällen der Strukturknoten, auf dem Gebiet $\Omega=\mathbb{R}^{3}$ spezifiziert. Die letzte Spalte 
zeigt den Separationsabstand $q_{X}$, also den kleinsten Abstand zwischen zwei Stützstellen.

Die Tabellen 20 bis 22 im Anhang A zeigen die rechnungsspezifischen Angaben zu den jeweiligen Abbildungen. In den Tabellen ist angegeben, welche Knoten als Stützstellen dienen, welcher Interpolationsalgorithmus verwendet wird sowie die Werte der wichtigsten Parameter. Eine detaillierte Beschreibung der Tabellen ist ebenfalls im Anhang A zu finden.

Die implementierten radialen Basisfunktionen sind in Tabelle 4 mit ihren in den nächsten Abschnitten verwendeten Abkürzungen zusammengestellt. Dabei ist zu beachten, dass die Funktionen EH und W1 bis W3 nur im $\mathbb{R}^{d}$ mit $d \leq 3$ benutzt werden können. Ferner gibt $m$ den Grad der bedingten Definitheit an. Die Angabe $m=0,1,2,3$ bedeutet, dass zur Bildung der Interpolanten keine, konstante, lineare oder quadratische Polynome benutzt werden müssen.

\begin{tabular}{|c||c|c|c|c|}
\hline$\phi(r)$ & Name & Abkürzung & $m$ & Glätte \\
\hline$r^{2} \log r$ & Thin Plate Spline & TPS & 2 & $C^{1}$ \\
$r^{4} \log r$ & Thin Plate Spline & TPS4 & 3 & $C^{3}$ \\
$r$ & Volume Spline & VS & 1 & $C^{0}$ \\
$r^{3}$ & Volume Spline & VS3 & 2 & $C^{2}$ \\
$\pi\left(\left(\frac{1}{12} r^{3}\right)-\left(R^{2} r\right)+\left(\frac{4}{3} R^{3}\right)\right)$ & Euklidischer Hut & EH & 0 & $C^{0}$ \\
$(1-r)_{+}^{2}$ & Wendland 1 & W1 & 0 & $C^{0}$ \\
$(1-r)_{+}^{4}(4 r+1)$ & Wendland 2 & W2 & 0 & $C^{2}$ \\
$(1-r)_{+}^{6}\left(35 r^{2}+18 r+3\right)$ & Wendland 3 & W3 & 0 & $C^{4}$ \\
$e^{-r^{2}}$ & Gauß & GA & 0 & $C^{\infty}\left(\mathbb{R}^{d}\right)$ \\
\hline
\end{tabular}

Tabelle 4. Die Aufstellung der implementierten radialen Basisfunktionen und ihrer in den folgenden Abschnitten verwendeten Abkürzungen.

Für die Visualisierung der Ergebnisse wurde das kommerzielle Softwarepaket AVS/Express (Advanced Visual Systems) genutzt. In den Abbildungen sind nur im Abschnitt 7 Einheiten angegeben, da in allen anderen Abschnitten die Physik der gezeigten Kopplungen nicht beschrieben wird und auch für diese Arbeit keine Relevanz hat.

\section{Anwendungen des parallelen Interfaces}

\subsection{Strömung-Struktur-Kopplung am AGARD Flügel}

Zur Demonstration der Schnittstelle der Interpolationsbibliothek zu MpCCI und damit auch zur Präsentation der Schnittstelle in einer parallelen Situation, die über MpCCI durch das verteilte Strömungsmodell im parallelen Strömungscode vorgegeben wird, wird ein aeroelastisches Standardmodell verwendet. Der AGARD-Wing 445.6 ist ein Holzmodell, welches in [24] 
definiert ist. Der Flügel hat eine Spannweite von $76 \mathrm{~cm}$ und ist um 45 Grad nach hinten gepfeilt.

Die zu interpolierenden Werte sind aus einer bereits berechneten Kopplung mit Standardinterpolationen hervorgegangen. Die Interpolationsalgorithmen sind bei dieser Anwendung nur von zweitrangigem Interesse, hauptsächlich soll mit diesem Beispiel das Interface in einer parallelen Situation demonstriert werden.

Die Deformationen werden bei diesem Anwendungsbeispiel mit radialen Basisfunktionen von den Strukturknoten auf die Strömungsknoten interpoliert. Die transponierte Matrix der daraus resultierenden Kopplungsmatrix wird zur Transformation der Kräfte von den Strömungsknoten auf die Strukturknoten verwendet.

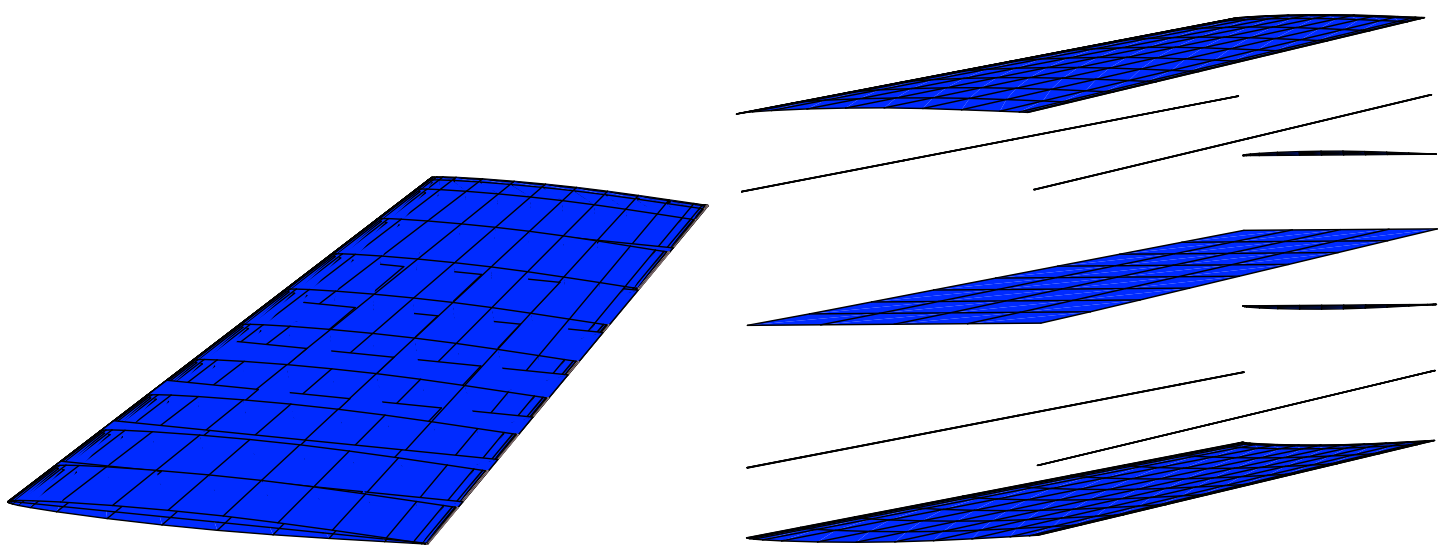

Abbildung 20. Links: Das Struktur- und Strömungsgitter. Rechts: Das Strömungsgitter wurde zusätzlich in seine Komponenten zerlegt.

In Abbildung 20 links sind das Struktur- und Strömungsgitter in ihrer gegenseitigen Lage abgebildet. Das Strukturgitter beschreibt eine fast ebene Platte, das Strömungsgitter eine die Strukturplatte umschließende, flügelförmige Geometrie. Wie in Abbildung 20 rechts zu erkennen ist, ist das Strömungsmodell des AGARD-Flügels auf acht Partitionen verteilt. Die Grafik zeigt die acht Partitionen, die durch Verschiebungen gegeneinander kenntlich gemacht werden sollen, zusammen mit dem Strukturgitter. Das Strukturgitter hat 45 Knoten und das Strömungsgitter 872 Knoten, aufgeteilt auf vier Partitionen mit 65 Knoten, zwei Partitionen mit 85 Knoten und zwei Partitionen mit 221 Knoten. Von diesen Knoten liegen natürlich einige an den Partitionsgrenzen und sind damit identisch zu anderen Knoten.

Tabelle 5 enthält die geometrischen Informationen dieses Datensatzes. Bereits hier ist ein typisches Phänomen zu erkennen, dass nämlich die gegebenen Datensätze hochgradig nicht uniform sind, d.h. dass der Separationsabstand $q_{X}$ wesentlich kleiner als die Fülldichte $h_{X, \Omega}$ ist. 


\begin{tabular}{|c|c|c|c|c|}
\hline $\begin{array}{c}\text { Anzahl } \\
\text { Strukturknoten }\end{array}$ & $\begin{array}{c}\text { Anzahl } \\
\text { Strömungsknoten }\end{array}$ & Stützstellen & $h_{X, \Omega}$ & $q_{X}$ \\
\hline 45 & 872 & Strukturknoten & $4.951906 e-02$ & $4.118022 e-07$ \\
\hline
\end{tabular}

Tabelle 5. Die Modellgrößen der Anwendung.

Die zu interpolierende Deformation auf dem Strukturgitter und das zu transportierende Kraftfeld auf dem Strömungsgitter sind in der Abbildung 21 dargestellt.
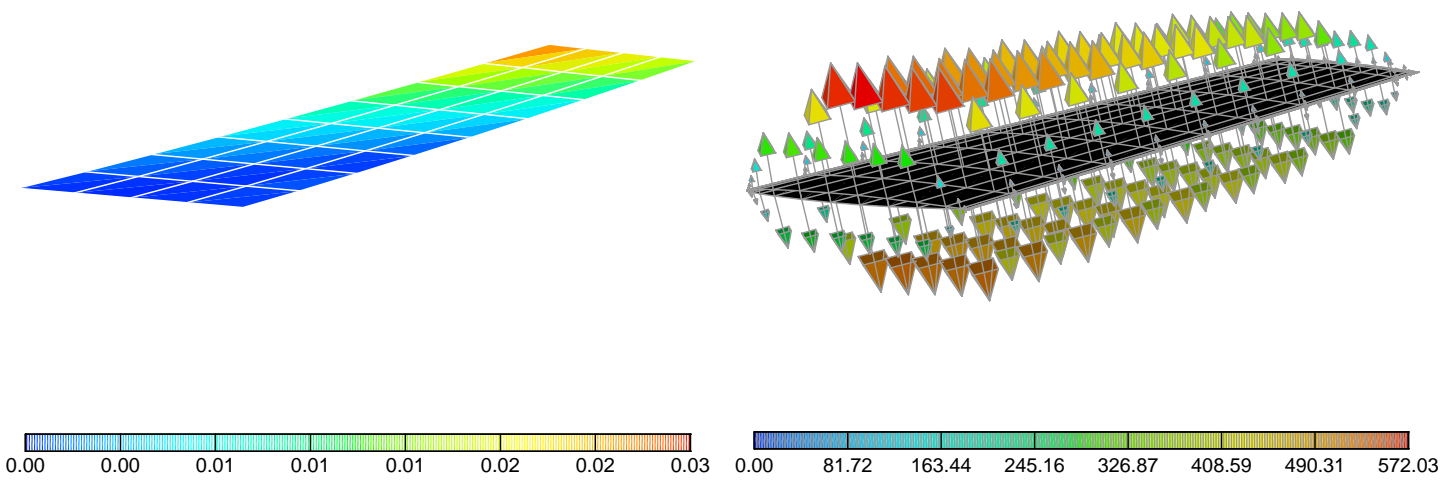

Abbildung 21. Links: Die gegebene Deformation auf dem Strukturgitter. Rechts: Das gegebene Kraftfeld auf dem Strömungsgitter.

In den Abbildungen 22 bis 29 sind die Ergebnisse der Kopplung für verschiedene Basisfunktionen gezeigt (siehe auch Tabelle 20 im Anhang A). Dabei ist links die interpolierte Verschiebung auf dem Strömungsgitter und rechts die durch die transponierte Kopplungsmatrix gegebene Kräfteverteilung auf dem Strukturgitter zu sehen.
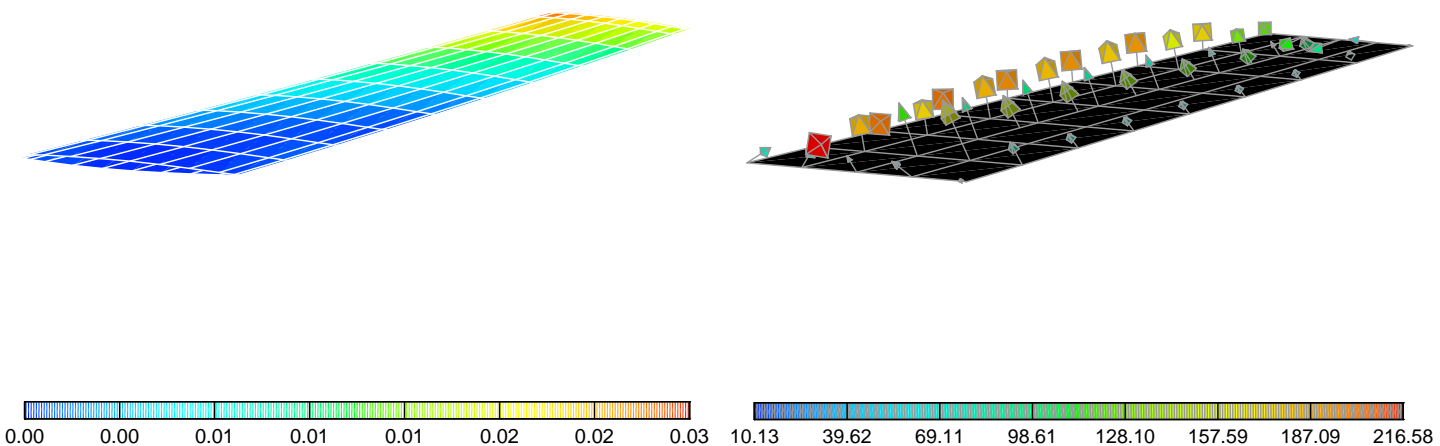

Abbildung 22. Die Ergebnisse für die Kopplung unter Verwendung von TPS. 

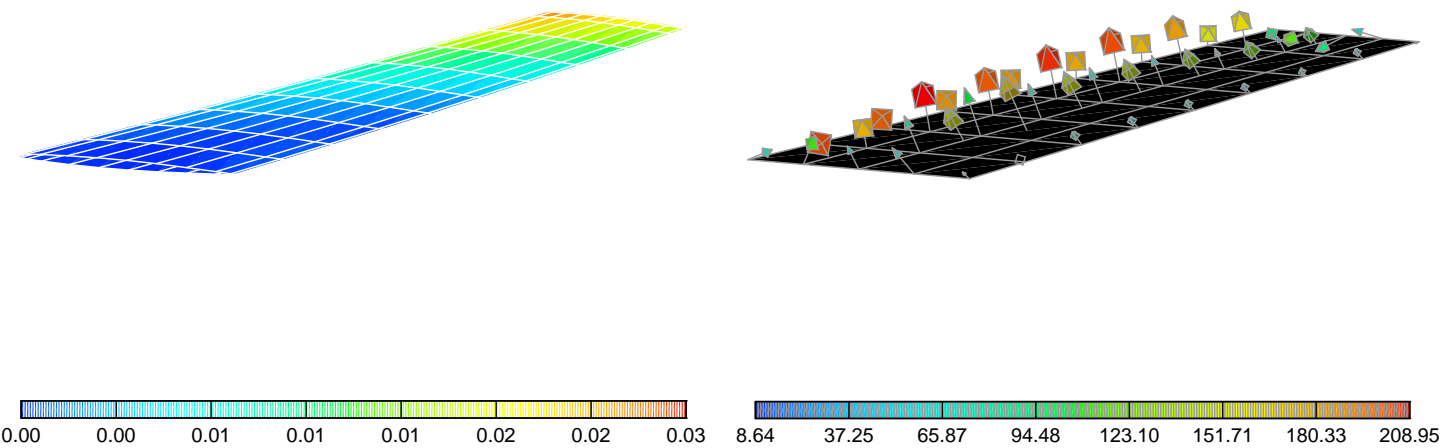

Abbildung 23. Die Ergebnisse der Kopplung unter Verwendung von TPS4.
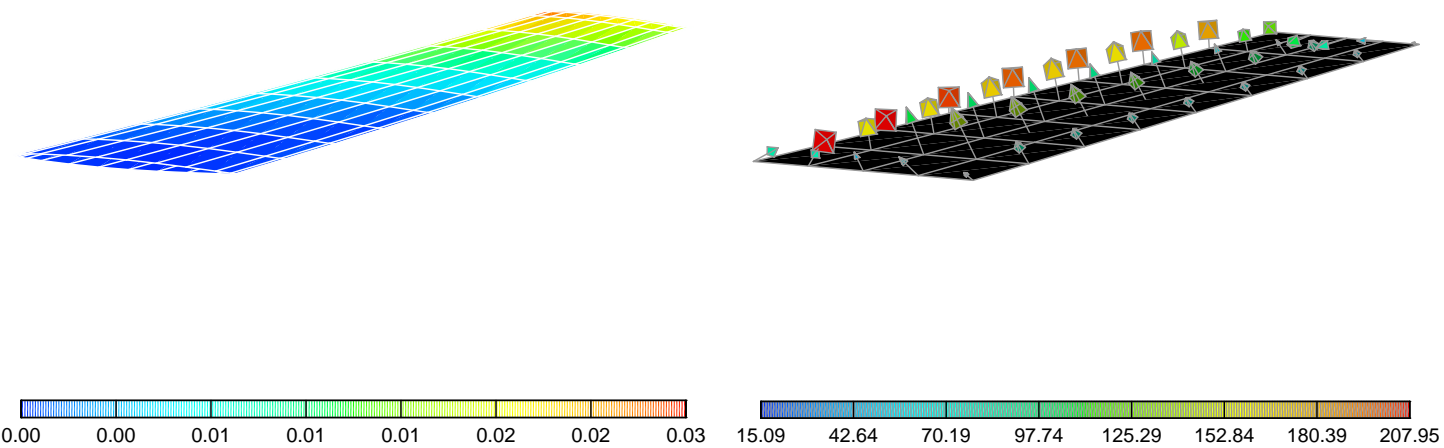

Abbildung 24. Die Ergebnisse der Kopplung unter Verwendung von VS.

Man erkennt, dass es zu sehr unterschiedlichen Kräfteverteilungen kommen kann, die teilweise für die praktische Rechnung nicht verwendbar sind.

Mit diesem parallelen Beispiel wird die Funktionalität der Schnittstelle von der Interpolationsbibliothek zu MpCCI demonstriert. Im Vergleich zu bereits durchgeführten Rechnungen zeigen die Resultate, dass die Funktionalität des Interfaces im parallelen Fall gegeben ist.
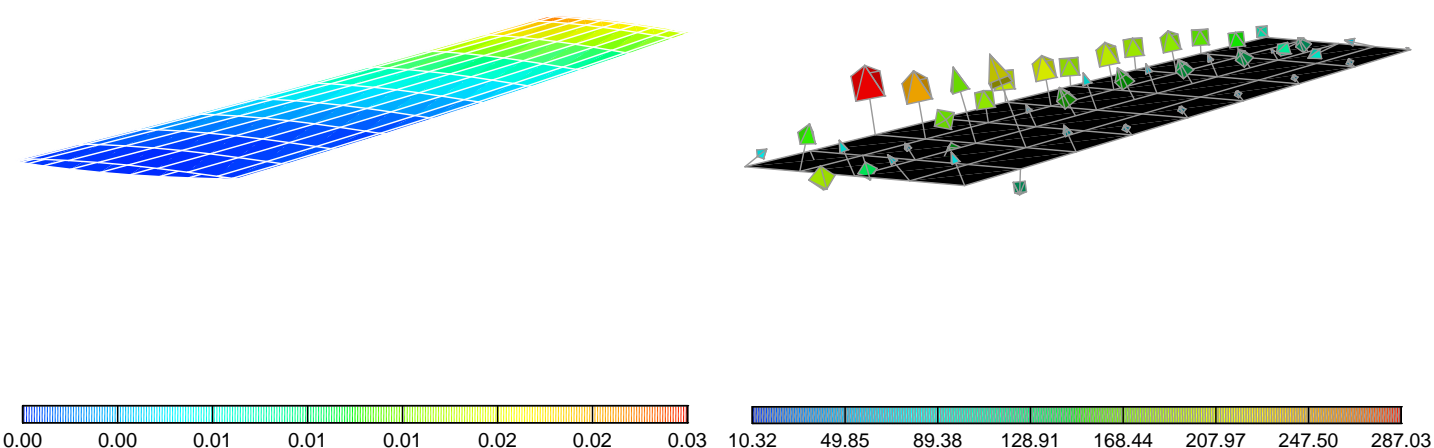

Abbildung 25. Die Ergebnisse der Kopplung unter Verwendung von VS3. 

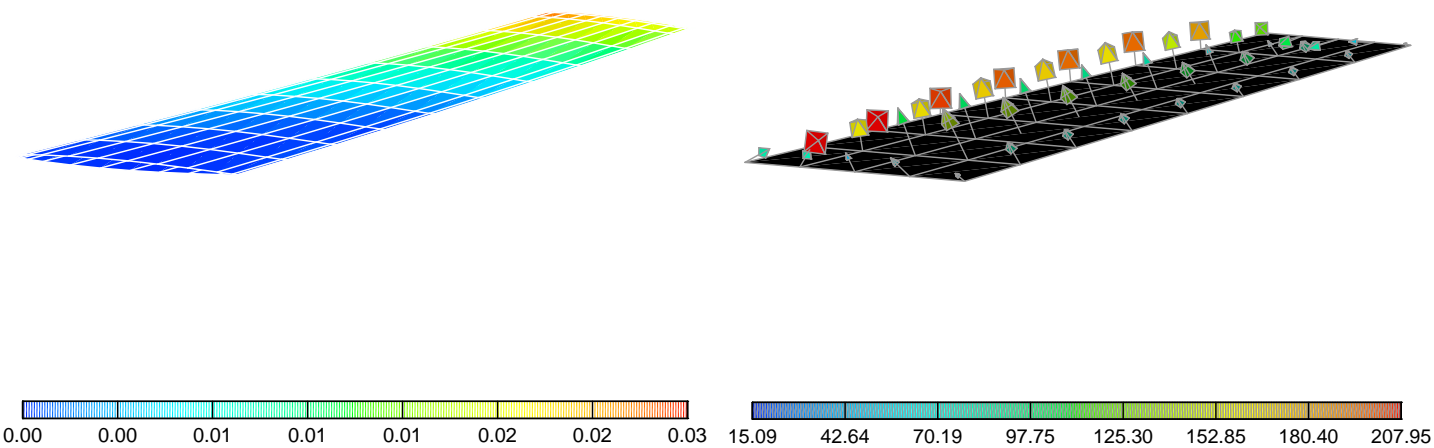

Abbildung 26. Die Ergebnisse der Kopplung unter Verwendung von EH.
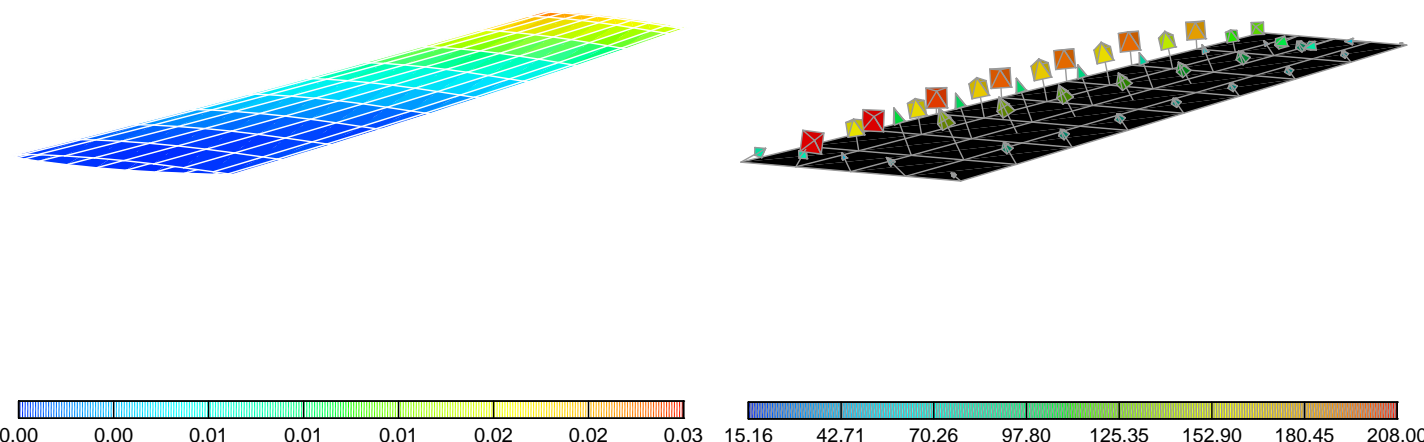

Abbildung 27. Die Ergebnisse der Kopplung unter Verwendung von W1.
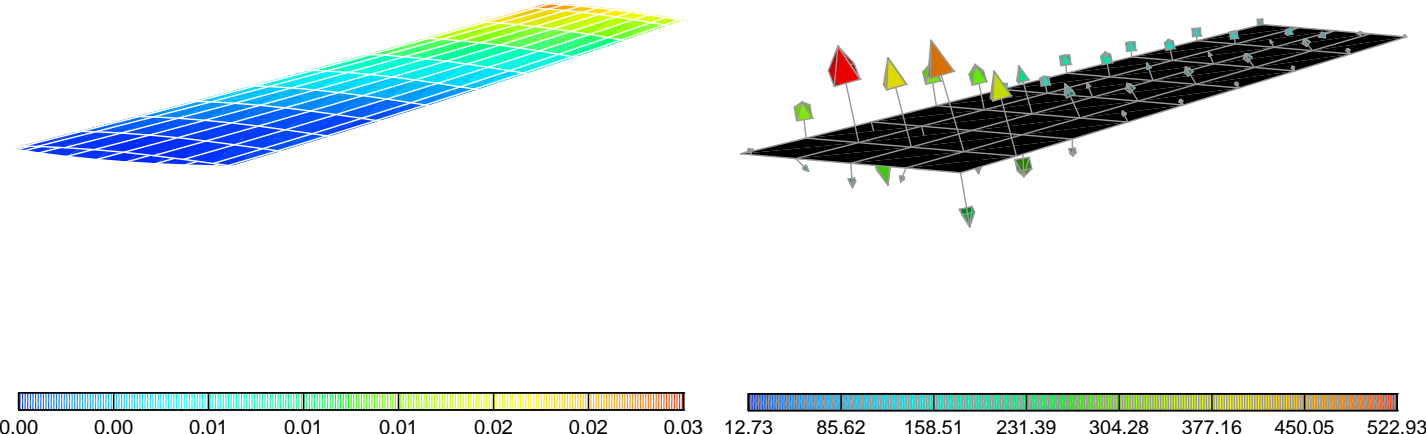

Abbildung 28. Die Ergebnisse der Kopplung unter Verwendung von W2.
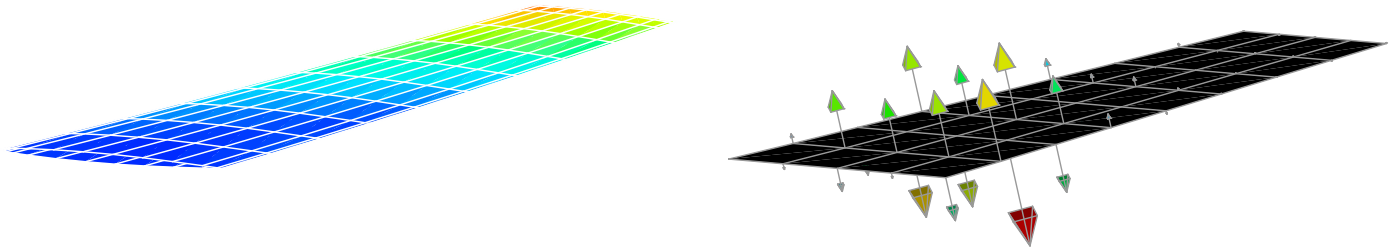

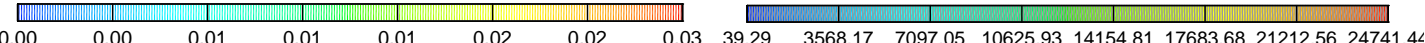

Abbildung 29. Die Ergebnisse der Kopplung unter Verwendung von W3. 


\section{Anwendungen unter Einbeziehung von Arbeitserhaltung und Konservativität}

\subsection{Strömung-Struktur-Kopplung am AGARD Flügel}

Es wird wieder der AGARD Flügel aus Abschnitt 1.1 betrachtet. Diesmal werden aber die Kräfte vom Strömungsgitter auf das Strukturgitter durch Interpolation mit Zusatzbedingungen übertragen. Die Zusatzbedingungen bestehen aus der Konservativität und der Erhaltung der Arbeit.

Sowohl die Gitter, wie auch die Ausgangsdeformationen und Ausgangskräfte sind bereits in Abschnitt 1.1 gezeigt worden. Die durch die Methoden ebenfalls identischen Ergebnisse der Interpolationen der Deformationen für die verwendeten radialen Basisfunktionen werden hier nicht ein zweites Mal präsentiert.

Die Abbildungen 30 bis 33 zeigen die über Interpolationen auf Grundlage radialer Basisfunktionen mit den Zusatzbedingungen zur Erhaltung der Arbeit und zur Konservativität erzielten Ergebnisse.

Diesmal sind die Kräfte insbesondere bei der Verwendung von VS, VS3, EH, und W1 einigermaßen gleich verteilt. Allerdings treten am Flügelrumpf negative Kräfte auf, die physikalisch wenig sinnvoll sind.

Da die Last kontinuierlich ist, wird sie mit der Methode der transponierten Kopplungsmatrix gleichmäßiger und sinnvoller verteilt.

Die entsprechenden Abbildungen 30 bis 33 sind ebenfalls in der Tabelle 21 im Anhang A, wie bei der ersten Berechnungsreihe in Abschnitt 1, beschrieben.
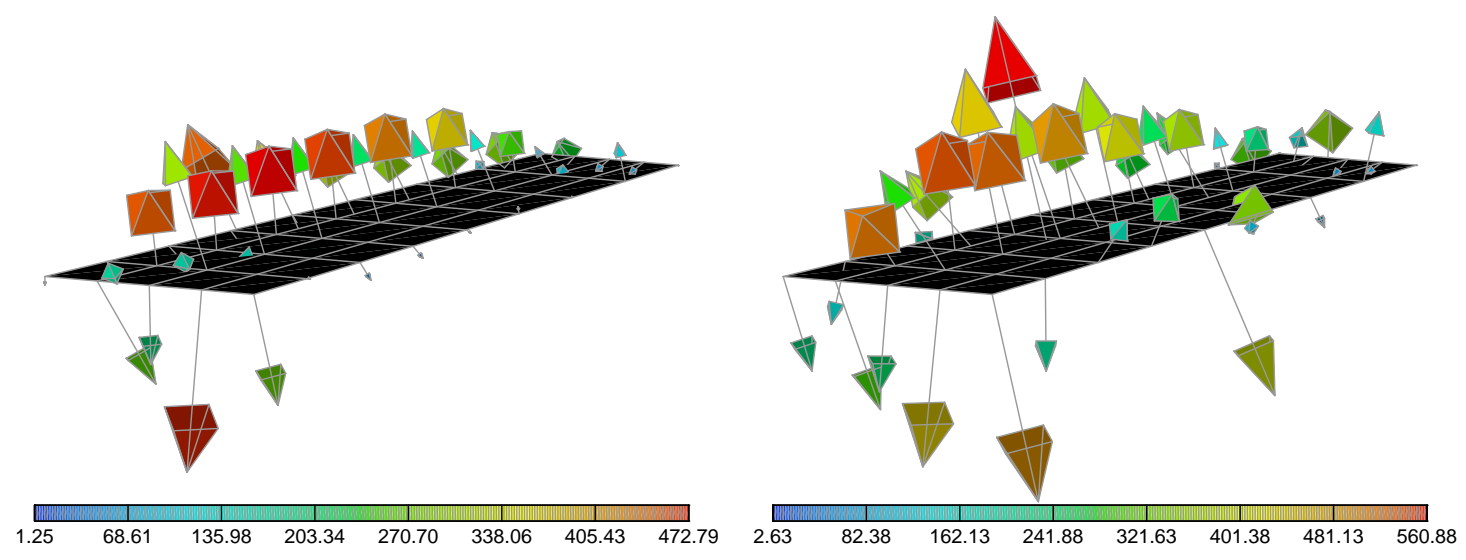

Abbildung 30. Die Ergebnisse der Interpolation unter Verwendung von TPS (links) und TPS4 (rechts). 

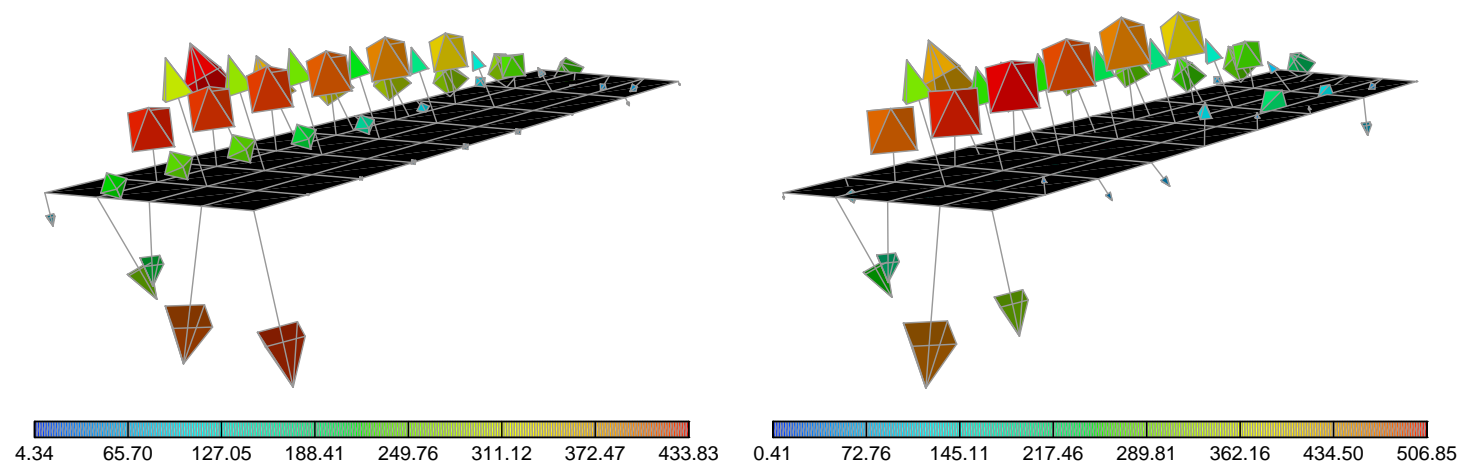

Abbildung 31. Die Ergebnisse der Interpolation unter Verwendung von VS (links) und VS3 (rechts).
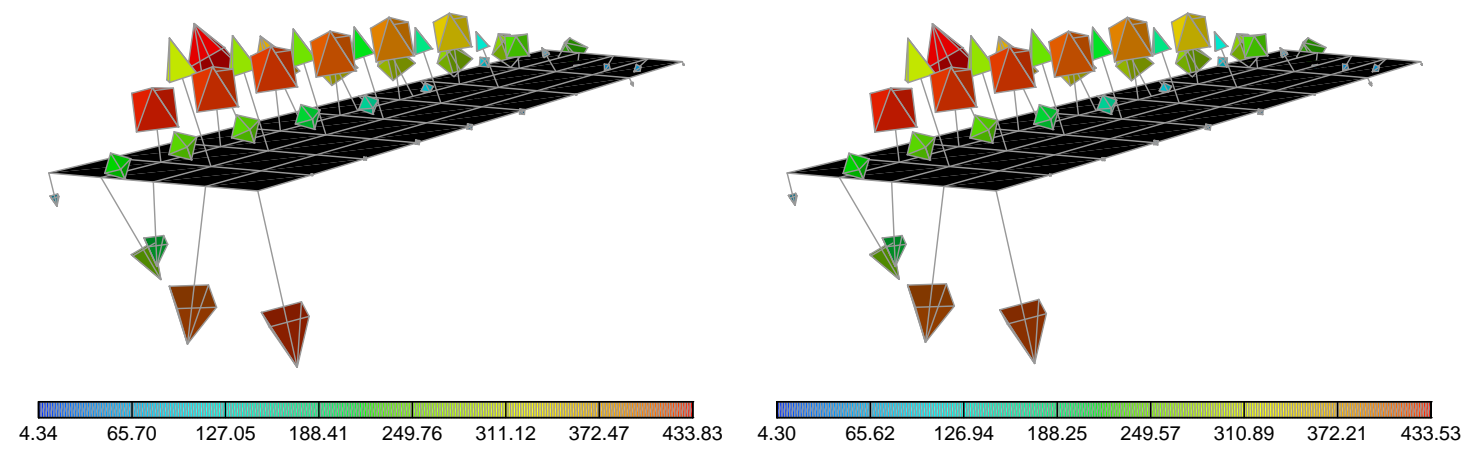

Abbildung 32. Die Ergebnisse der Interpolation unter Verwendung von EH (links) und W1 (rechts).
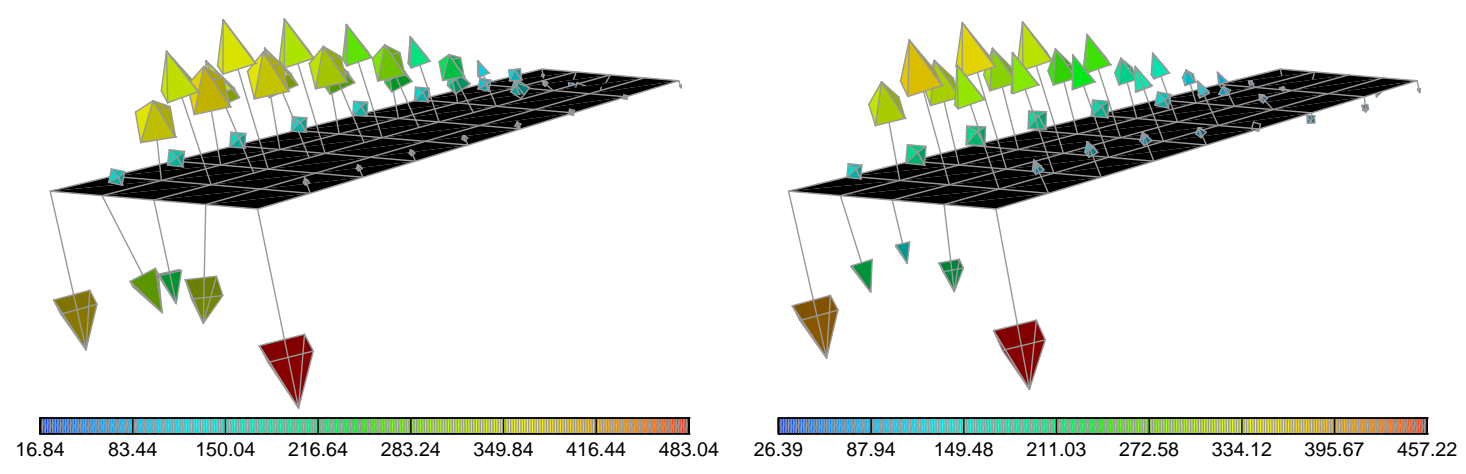

Abbildung 33. Die Ergebnisse der Interpolation unter Verwendung von W2 (links) und W3 (rechts). 


\section{Anwendungen unter Einbeziehung der Rotationen}

\subsection{Strömung-Struktur-Kopplung am AMP Flügel}

In diesem Abschnitt wird die Funktionalität der Interpolation mit radialen Basisfunktionen unter Einbeziehung der Rotationen über entsprechende $\mathrm{Zu}$ satzbedingungen demonstriert. Die Interpolation wird an dem AMP-Flügel [7] getestet, wobei das Strukturmodell nur aus einer Kette von 24 Balkenelementen besteht. Das Strömungsmodell dagegen besitzt allein auf der Oberfläche, die die Kopplungsfläche auf Strömungsseite ist, 109216 Dreieckselemente mit 54653 Knoten. Die zu interpolierenden Werte sind aus einer bereits berechneten Kopplung mit Standardinterpolationen hervorgegangen.
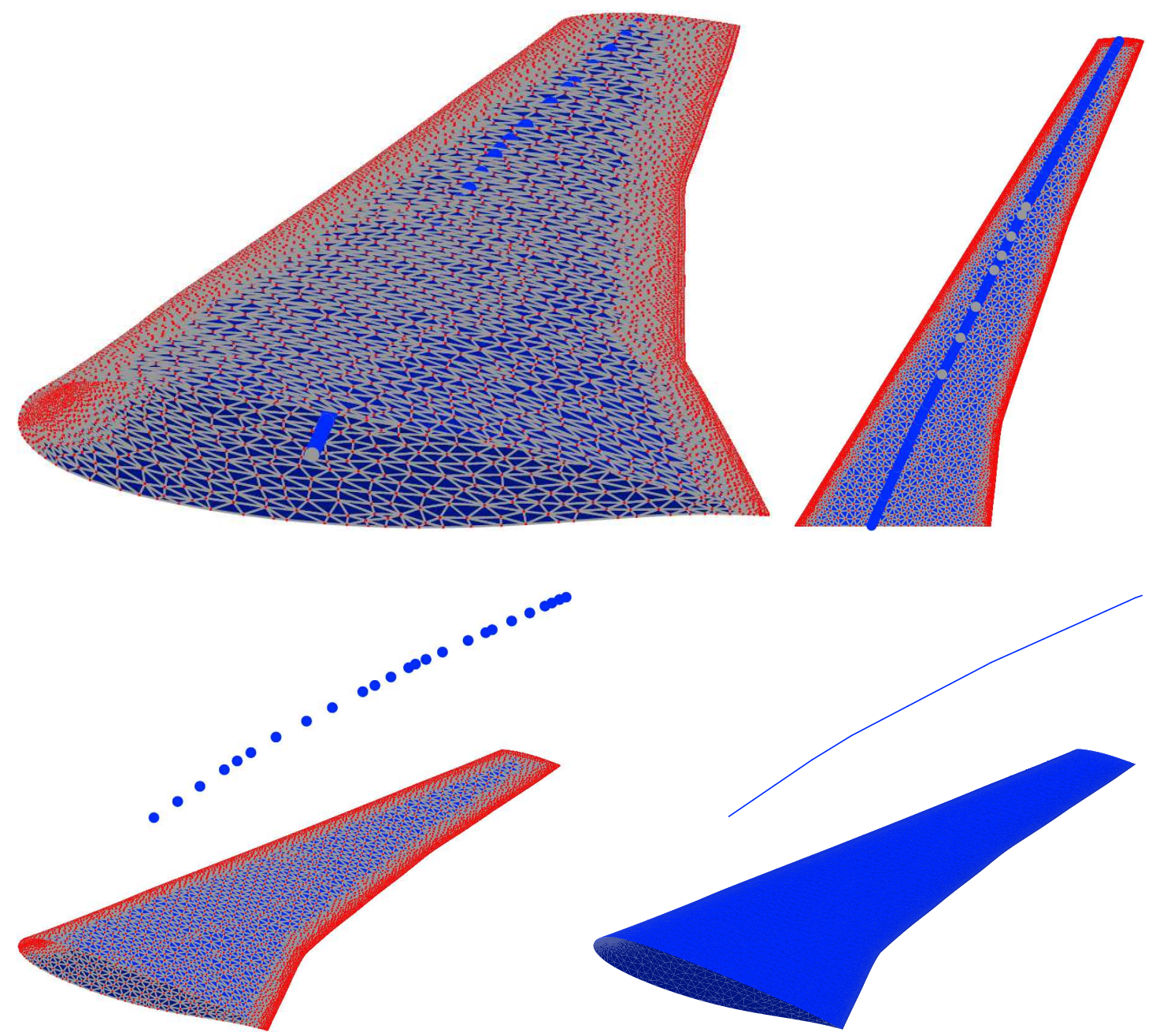

Abbildung 34. Das aus Balkenelementen bestehende Strukturmodell und das Strömungsmodell des AMP-Flügels.

Die Deformationen mit den Zusatzbedingungen bezüglich der Rotationen werden wie üblich bei dieser Art Anwendungsbeispiel von den Strukturknoten 
auf die Strömungsknoten interpoliert, so dass wieder die Strukturknoten die Stützstellen und die Strömungsknoten die Auswertungsstellen sind.

Dieses Beispiel eignet sich besonders für die Interpolation unter Einbeziehung der Rotationen, da auf Seite der Stützstellen durch die sehr geringe Anzahl von Knoten, besonders im Vergleich zu der Anzahl der Auswertungsstellen, sehr wenige Informationen für die Interpolation vorhanden sind. Die Nutzung der Rotationen als Zusatzinformationen für die Interpolationen ist also gerade bei einem solchen Beispiel von großem Interesse.

In Abbildung 34 sind das undeformierte Struktur- und Strömungsgitter des AMP-Flügels dargestellt; oben links mit der richtigen Lage zueinander und rechts sowie unten zur besseren Darstellung auseinander gezogen. Das Strukturgitter, ist jeweils oben zu sehen und das Strömungsgitter unten. Das Strukturgitter ist eine Kette von Balkenelementen und liegt innerhalb des Strömungsgitters, wie an der vorderen Öffnung des Strömungsgitters auf Abbildung 34 oben links zu sehen ist.

Die Tabelle 6 enthält wieder die anwendungsspezifischen Größen. Die Abbildungen zu den Interpolationen sind in der Tabelle 21 im Anhang A übersichtlich aufgelistet und spezifiziert, wie in Abschnitt 2.3 beschrieben.

\begin{tabular}{|c|c|c|c|c|}
\hline $\begin{array}{c}\text { Anzahl } \\
\text { Strukturknoten }\end{array}$ & $\begin{array}{c}\text { Anzahl } \\
\text { Strömungsknoten }\end{array}$ & Stützstellen & $h_{X, \Omega}$ & $q_{X}$ \\
\hline 25 & 54653 & Strukturknoten & $2.38915 e-01$ & $8.89221 e-03$ \\
\hline
\end{tabular}

Tabelle 6. Die Modellgrößen der Anwendung.

Die Ergebnisse der Interpolationen der Deformationen von den Strukturknoten auf die Strömungsknoten unter Einbeziehung der Rotationen werden in den Abbildungen 35 und 36 gezeigt. In den Abbildungen 37 und 38 werden die Ergebnisse der Interpolationen der gleichen Deformationen dargestellt, aber hier wurden die Rotationen nicht als Zusatzbedingungen in der Interpolation verwendet. Die Abweichungen der Ergebnisse beider Interpolationen sind in den Abbildungen 39 und $40 \mathrm{zu}$ sehen. Die Interpolation wurde in beiden Verfahren mit der Wendland 2 Funktion durchgeführt. Die Deformationen auf dem Strukturgitter, die die Ausgangslage der Interpolationen darstellen, und die interpolierten Deformationen auf den Strömungsknoten des AMP-Flügels werden nebeneinander dargestellt. Die vertikale Legende bezieht sich auf die gegebenen Deformationen und die horizontale auf die interpolierten Deformationen. Die Abbildungen zeigen 8 Schritte einer Simulation bei vorgegebenen Deformationen und Rotationen auf Strukturseite. Die maximalen Abweichungen zwischen den Interpolationsergebnissen mit und ohne Einbeziehung der Rotationen sind für jeden Schritt in der Tabelle 7 aufgelistet. Die Abweichungen sind, wie zu erwarten war, im mittleren Bereich des Flügels minimal und wachsen zur Vorder- und Hinterkante an. 
An der Vorder- und Hinterkante haben die Rotationen die größten Auswirkungen, da die Deformationen ohne die Rotationen auf den Strukturknoten so gut wie nur Informationen über den Verlauf in Richtung längs des Flügels haben. Quer zum Flügel, also von der Mitte in Richtung Vorder- und Hinterkante, sind die Informationen bei dieser Anordnung der Strukturknoten nur durch die Rotationen zu gewinnen.

Bei Interpolation einer in Richtung der Höhe des Flugzeugs, entlang des Flügels aufgetragenen, quadratischen Funktion, ist der maximale Fehler zu den aufgetragenen Funktionwerten auf den Strömungsknoten unter Einbeziehung der Rotationen etwa 63\% des maximalen Fehlers bei Interpolation ohne Verwendung der Rotationen. Wird die quadratische Funktion entlang des Flugzeugrumpfs aufgetragen, ist der auf die gleiche Weise berechnete maximale Fehler unter Einbeziehung der Rotationen etwa 40\% des maximalen Fehlers bei der Interpolation ohne Verwendung der Rotationen.

\begin{tabular}{|c|c|}
\hline Schritt & Abweichung \\
\hline 1 & $2.864 e-03$ \\
2 & $2.665 e-03$ \\
3 & $3.084 e-03$ \\
4 & $3.366 e-03$ \\
5 & $3.291 e-03$ \\
6 & $3.320 e-03$ \\
7 & $3.311 e-03$ \\
8 & $3.319 e-03$ \\
\hline
\end{tabular}

Tabelle 7. Die maximalen Abweichungen zwischen den Interpolationsergebnissen.

Da auch hier keine experimentellen Daten zum Vergleich vorliegen, kann wieder nur durch die Visualisierung die Anwendbarkeit der Interpolation unter Einbeziehung der Rotationen demonstriert werden.

Da die gegebenen Deformationen und Rotationen aber aus einer konvergierenden Rechnung stammen, sollten auch die interpolierten Deformationen konvergieren, was insbesondere an der Flügelspitze zu erkennen ist.

Da sich Rotationen nur für kleine Winkel in Ableitungen übersetzen lassen, ist die Anwendung der Interpolation mit Rotationen auch auf solche beschränkt, was in der Aeroelastik aber kein Problem ist. Da es sich hier aber um einen Fall extremer Extrapolation handelt, sind die Ergebnisse immer kritisch zu betrachten. Sinnvoll wäre es z.B. auch, die interpolierten Rotationen zu benutzen, um durch zusätzliche starre Balkenansätze eine Kopplung zu erwirken. 

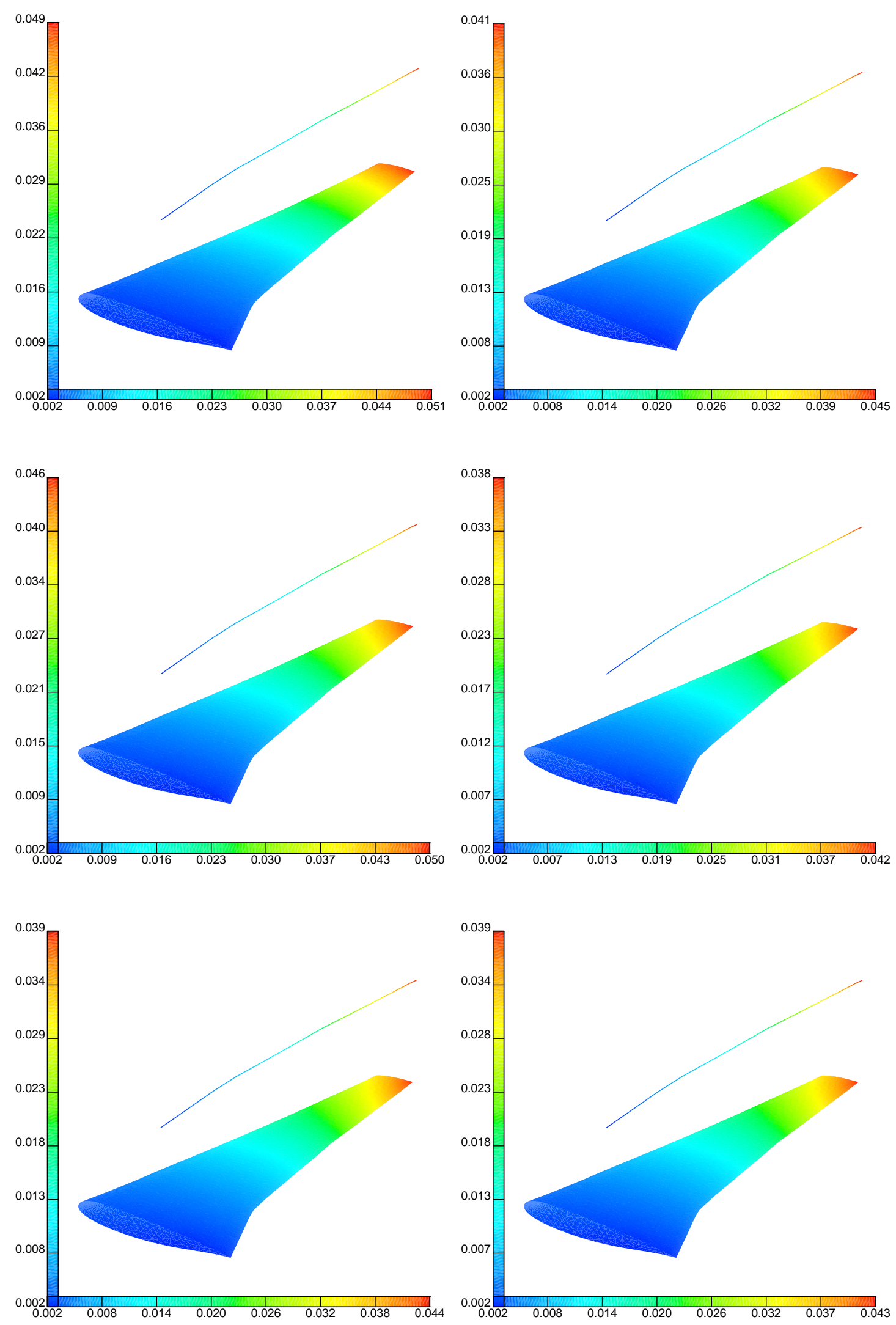

Abbildung 35. Die gegebenen und interpolierten Deformationen (Schritte 1-6). Die Ergebnisse der Interpolation unter Verwendung von W2 wurden mit Rotationen als Zusatzbedingungen erzielt. 

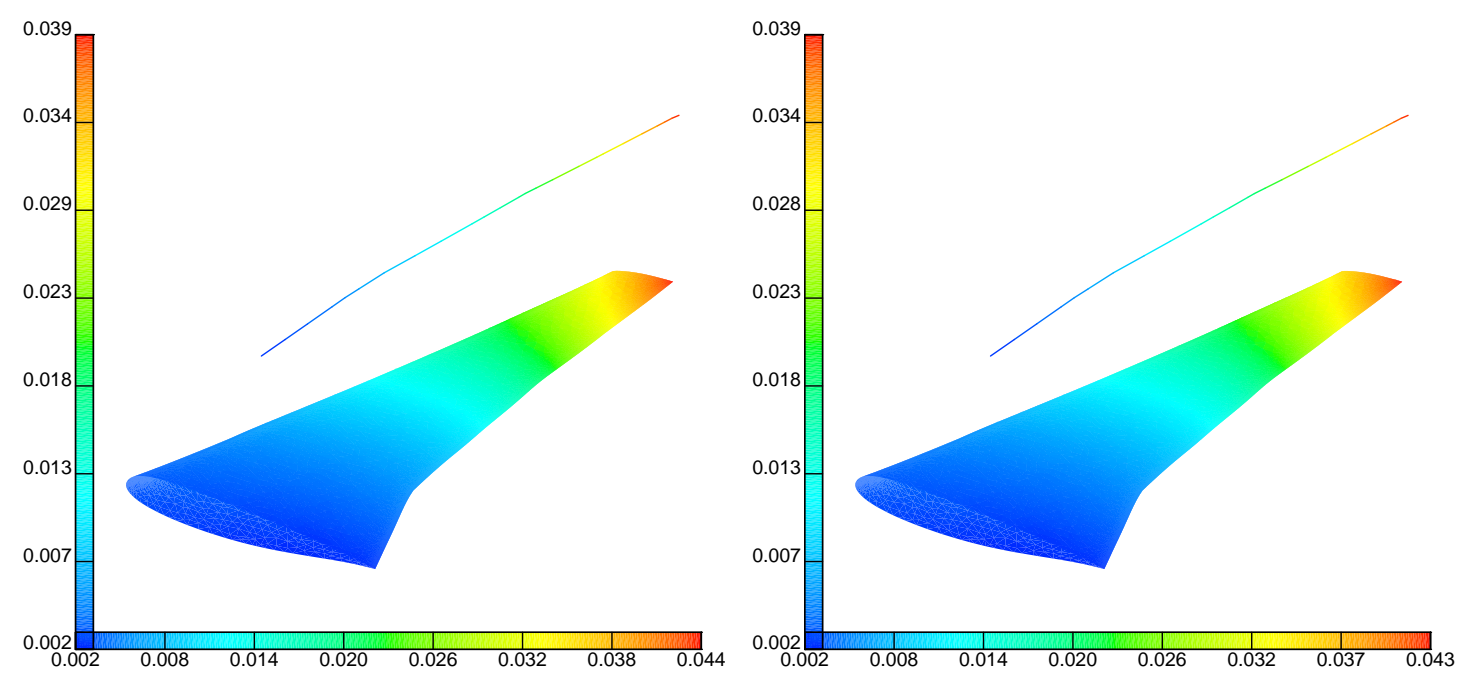

Abbildung 36. Die gegebenen und interpolierten Deformationen (Schritte 7 u. 8). Die Ergebnisse der Interpolation unter Verwendung von W2 wurden mit Rotationen als Zusatzbedingungen erzielt.
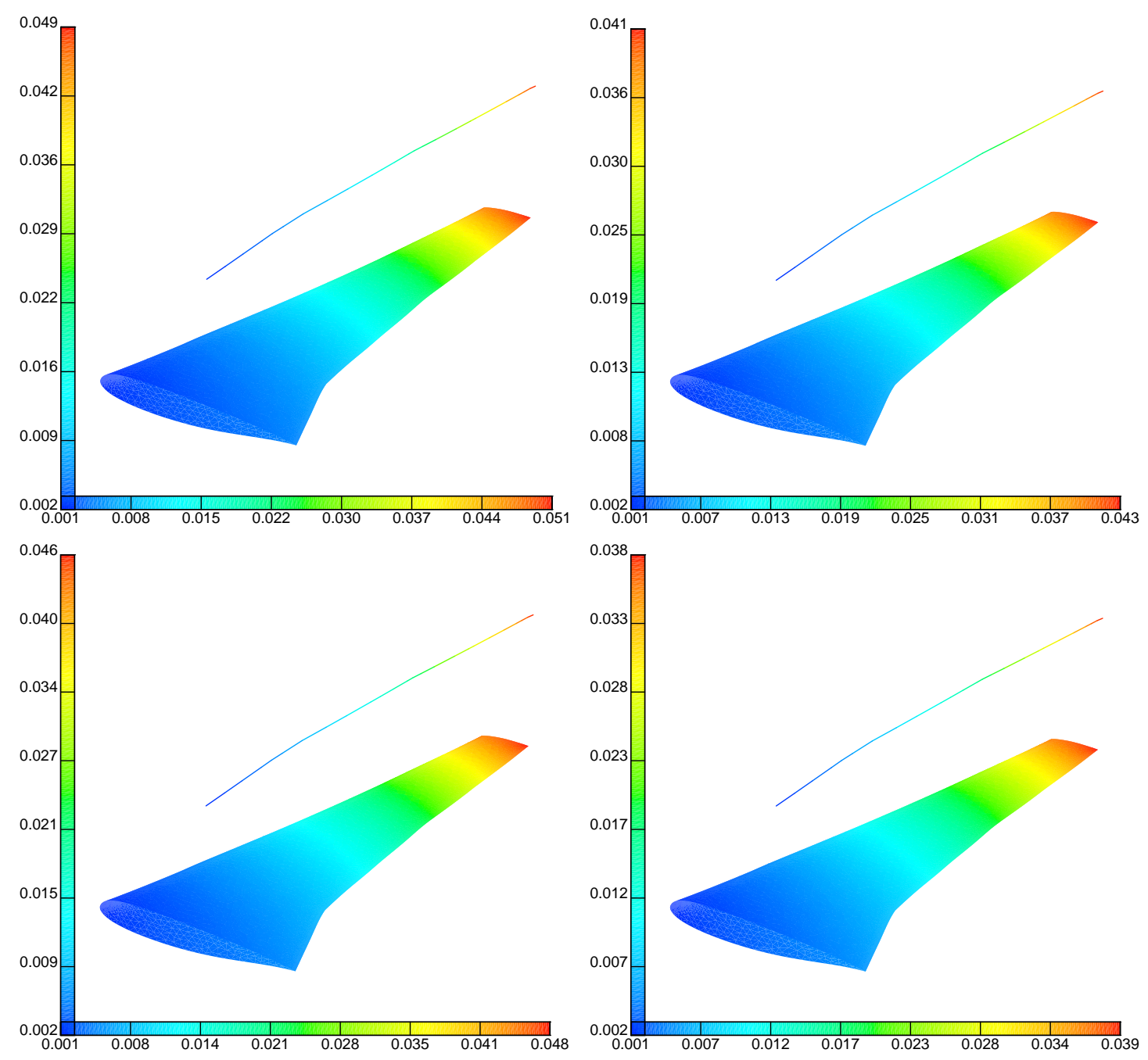

Abbildung 37. Die gegebenen und interpolierten Deformationen (Schritte 1-4). Die Ergebnisse der Interpolation unter Verwendung von W2 wurden ohne Rotationen als Zusatzbedingungen erzielt. 

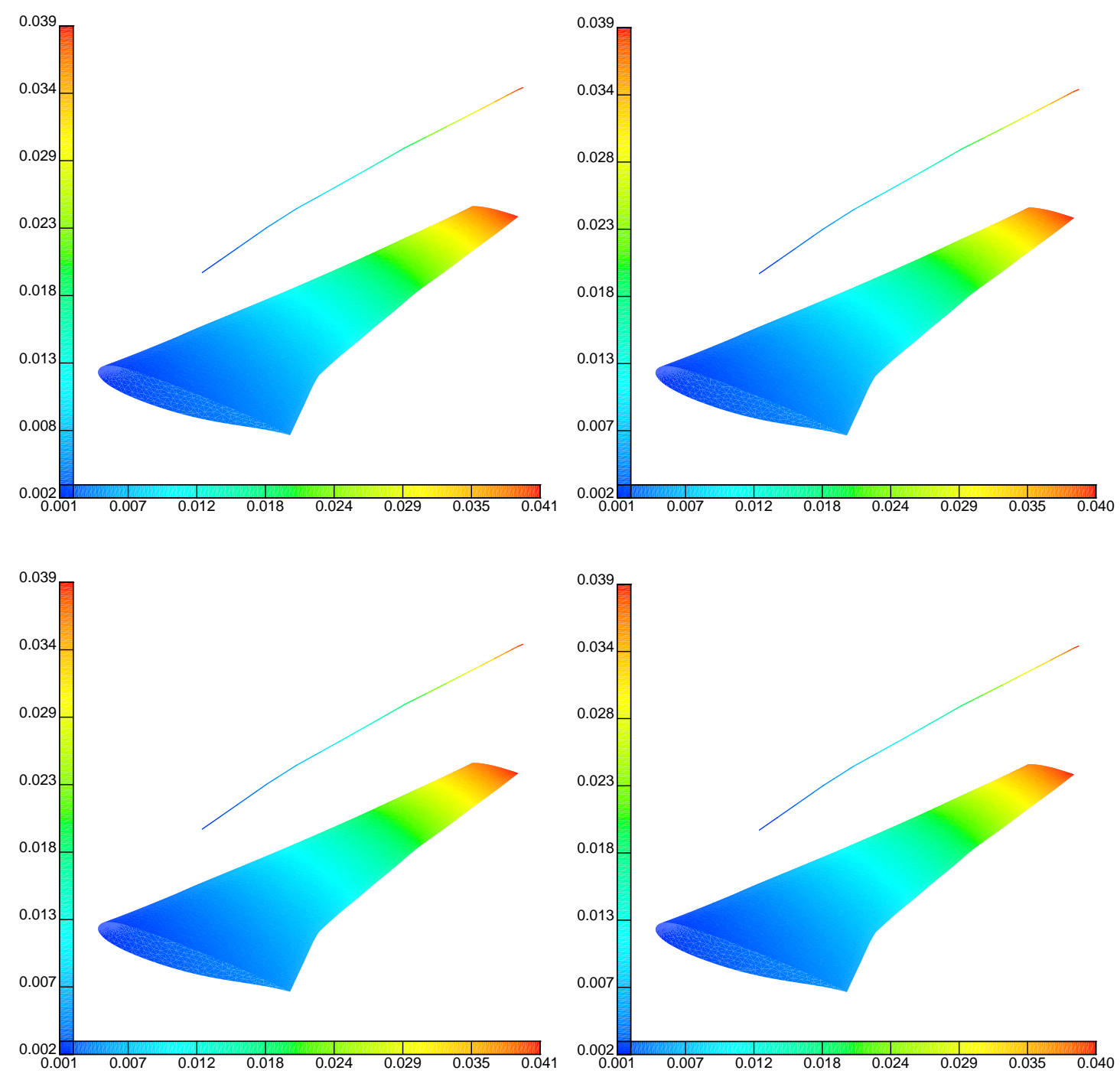

Abbildung 38. Die gegebenen und interpolierten Deformationen (Schritte 5-8). Die Ergebnisse der Interpolation unter Verwendung von W2 wurden ohne Rotationen als Zusatzbedingungen erzielt.
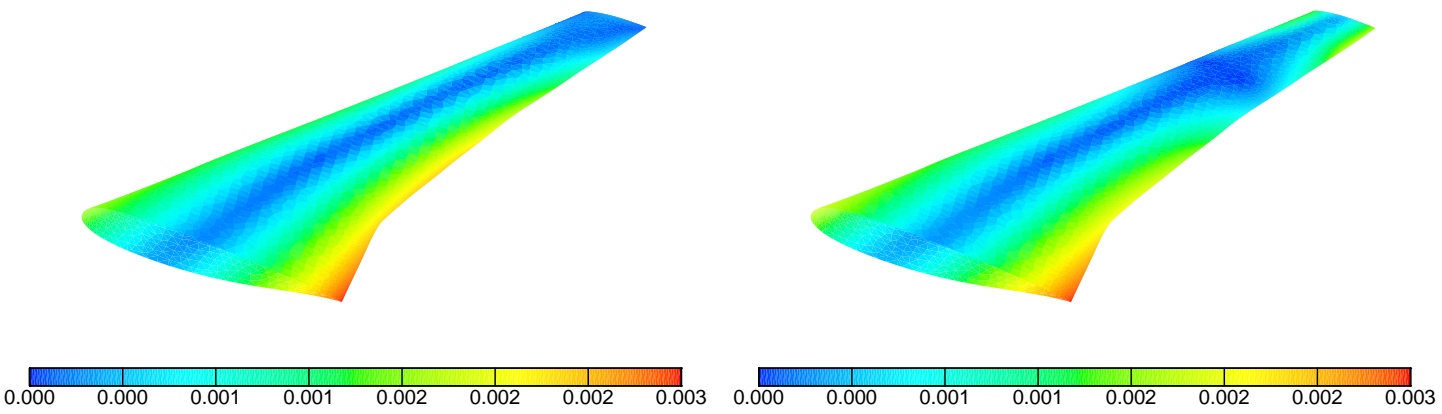

Abbildung 39. Die Abweichungen zwischen den Ergebnissen der interpolierten Deformationen mit und ohne Rotationen als Zusatzbedingungen (Schritte 1 u. 2). Die Deformationen wurden in beiden Verfahren unter Verwendung von W2 interpoliert. 

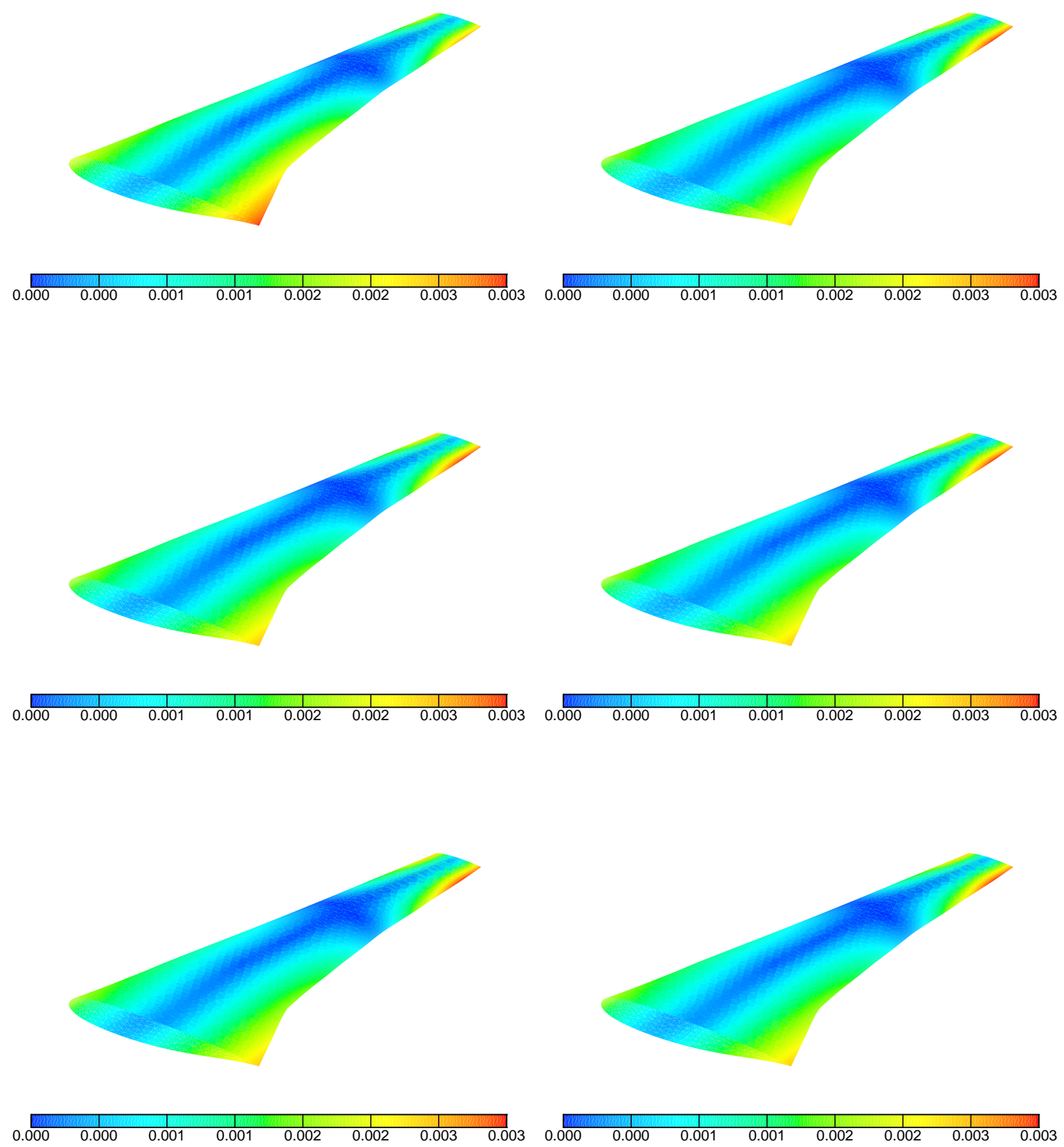

Abbildung 40. Die Abweichungen zwischen den Ergebnissen der interpolierten Deformationen mit und ohne Rotationen als Zusatzbedingungen (Schritte 3-8). Die Deformationen wurden in beiden Verfahren unter Verwendung von W2 interpoliert. 


\section{Anwendungen des „Partition of Unity“ Verfahrens}

Es soll nun das Partition of Unity Verfahren an verschiedenen Problemen getestet werden. Dabei wird neben dem Austausch von Verschiebungen zwischen Strömungs- und Strukturmodell eines Flugzeuges auch der Datenaustausch zwischen verschiedenen Rohrleitungsmodellen, bei der Sprühlackierung und in der Umformtechnik betrachtet.

\subsection{Das A340-300 Halbmodell - Ein analytischer Testfall}

Das folgende Testbeispiel soll die Anwendbarkeit der „Partition of Unity“ Methode auf die Deformationen einer Flugzeugstruktur zeigen. Dazu wird eine wenig realistische Deformation in Form einer Sinuskurve entlang des Flügels angenommen. Auch wenn diese Deformation weit entfernt von realen Konfigurationen ist, zeigt sie tendenziell die Probleme bei derartigen Kopplungsverfahren auf.
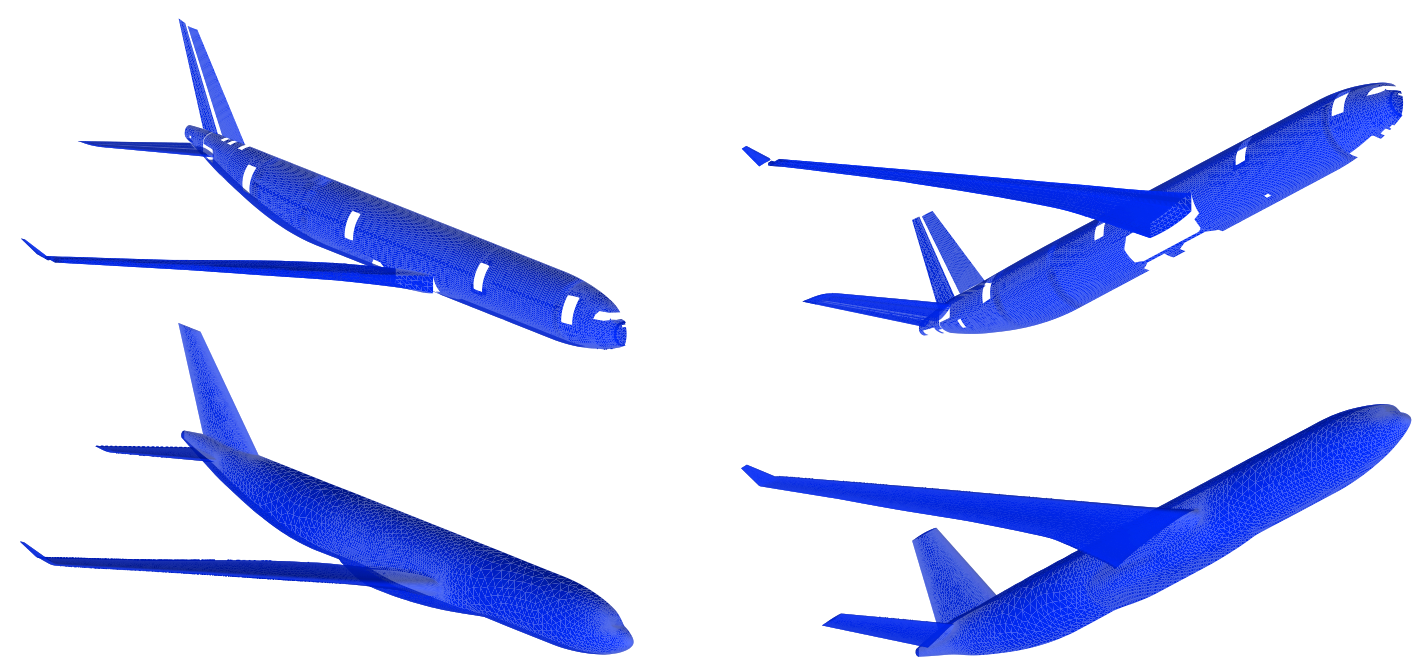

Abbildung 41. Das Strömungsmodell (unten) und Strukturmodell (oben) des A340-300.

In den Abbildungen 41 und 42 sind das undeformierte Struktur- und Strömungsgitter des A340 dargestellt. Abbildung 41 zeigt beide Modelle einzeln aus verschiedenen Perspektiven, während Abbildung 42 die Lage der Gitter zueinander darstellt. Zum besseren Verständnis wurde hier das Strukturgitter als Gitter dargestellt, während das Strömungsmodell wieder schattiert wurde. Man erkennt, dass z.B. der Flügel des Strukturmodells nur etwa die halbe Breite des Flügels des Strömungsmodells hat. Dies bedeutet, dass für die Verschiebungen auf Strömungsseite die Auswertung der Interpolanten außerhalb des Stützstellenbereichs erfolgen muss. Es handelt sich also eigentlich um eine Extrapolation. Diese Tatsache bereitet vielen Kopplungsverfahren Probleme und hat auch hier Einfluss auf die Wahl der Parameter, wie in den folgenden Beispielrechnungen demonstriert wird. 


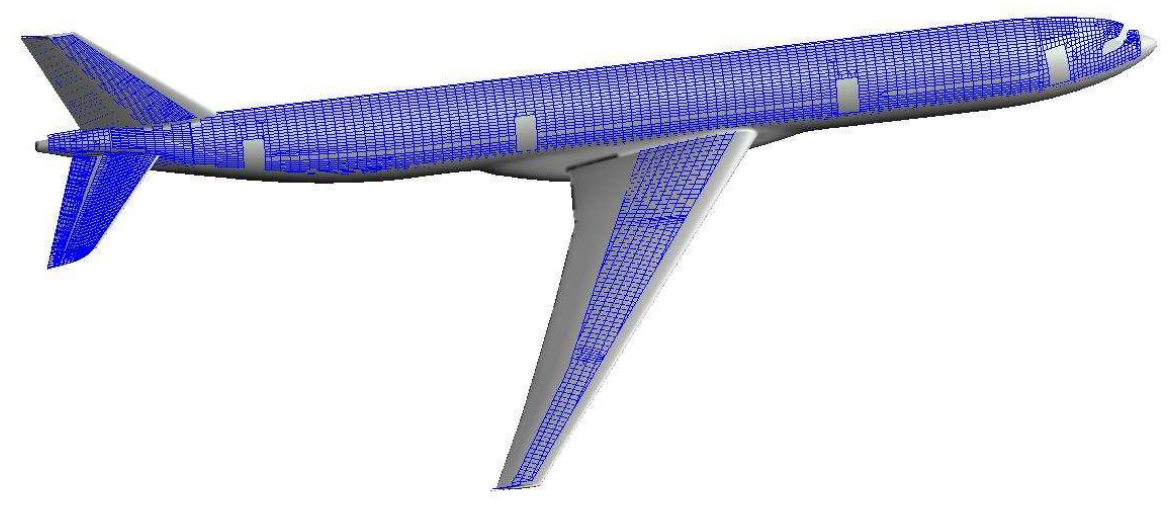

Abbildung 42. Das Strömungs- und Strukturmodell des A340-300.

Die im folgenden präsentierten Abbildungen mit den Interpolationsergebnissen sind wie in den vorherigen Abschnitten in der Tabelle 21 im Anhang A mit den Spezifikationen zur Interpolation aufgelistet. Tabelle 8 enthält die übrigen modellrelevanten Größen.

\begin{tabular}{|c|c|c|c|c|}
\hline $\begin{array}{c}\text { Anzahl } \\
\text { Strukturknoten }\end{array}$ & $\begin{array}{c}\text { Anzahl } \\
\text { Strömungsknoten }\end{array}$ & Stützstellen & $h_{X, \Omega}$ & $q_{X}$ \\
\hline 11893 & 33444 & Strukturknoten & 2.95878 & $1.43 e-06$ \\
\hline
\end{tabular}

Tabelle 8. Die Modellgrößen der Anwendung.

Wie bereits erwähnt, wird die hier betrachtete Deformation durch eine Sinuskurve gegeben, die auf das Strukturgitter längs des Flügels in Richtung der Höhe des Flugzeugs aufgebracht wird (siehe Abbildung 43).

Um einen prinzipiellen Vergleich des Interpolationsverfahrens mit der PoU Methode zu haben, wurde dieses Beispiel zunächst mit einem globalen Ansatz durchgerechnet. Dazu wurde als Basisfunktion der Euklidische Hut (EH) mit unterschiedlicher Trägerweite benutzt. Als iterativer Löser kam BiCGStab zum Einsatz. Die Ergebnisse sind in Abbildung $44 \mathrm{zu}$ sehen. Dabei zeigt die linke Seite die Rekonstruktion basierend auf jeweils mindestens 5 Strukturknoten im Trägerbereich, während es auf der rechten Seite mindestens 40 Strukturknoten sind. Die obere Zeile zeigt die Rekonstruktion ohne jegliche polynomiale Terme, wogegen in der unteren Zeile jeweils quadratische Polynome zur Bildung der Interpolanten benutzt wurden. 

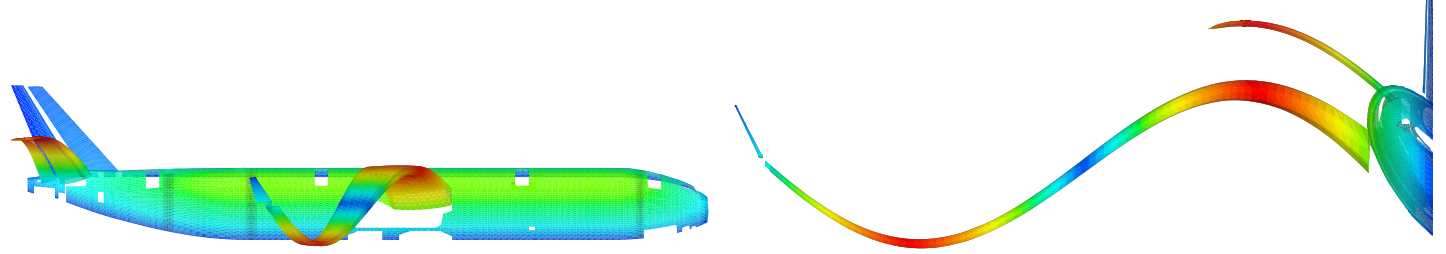

Abbildung 43. Sinusartige Deformation entlang des Flügels.
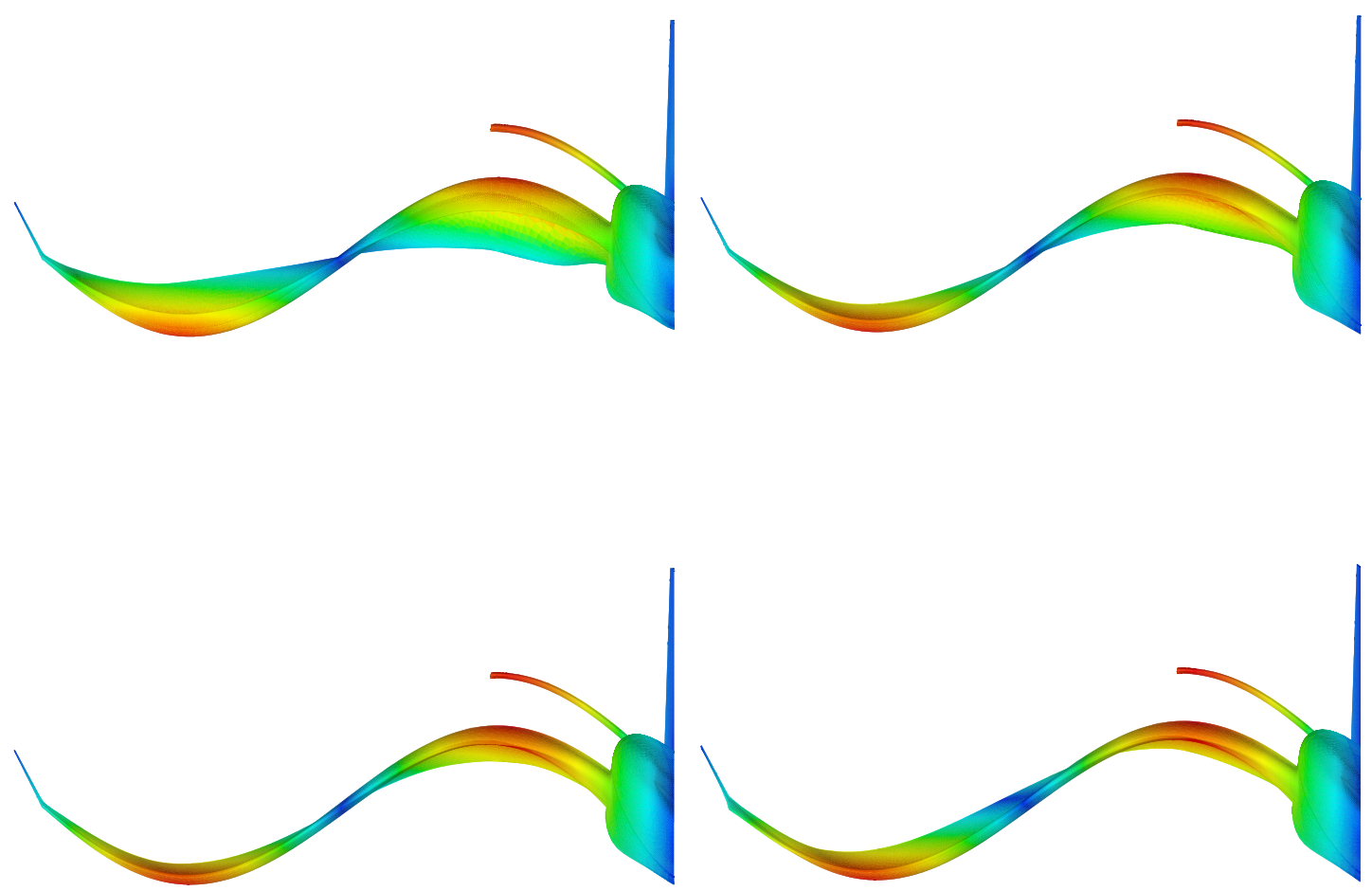

Abbildung 44. Interpolation der sinusförmigen Deformation mit EH. Links sind jeweils mindestens 5 Strukturknoten im Trägerbereich, rechts sind es mindestens 40. Oben wurden keine polynomialen Terme genutzt, unten quadratische.

Man erkennt im hinteren Bereich des Flügels die typischen Extrapolationsprobleme. Der kompakte Träger des Euklidischen Huts sorgt bei zu geringer Trägerwahl dafür, dass an der hinteren Kante des Flügels keine Deformationsinformation mehr ankommt. Eine größere Trägerwahl verbessert das Ergebnis auf Kosten längerer Rechenzeit. Bessere Ergebnisse lassen sich mit einem zusätzlichen globalen Term erzielen.

Ähnliche Probleme treten auch beim verwendeten PoU Verfahren auf. Wenn die Partitionen zu klein gewählt werden, liegen die Auswertungsstellen außerhalb des Einflussbereichs der Interpolanten, so dass diese dort keine Information mehr liefert. Durch hinreichend große Buckets, wie z.B. in Abbildung 45 links oben zu sehen, werden aber gute Rekonstruktionen erhalten.

Die dreidimensionale Aufteilung erfolgte hier in 16.7.7 überlappende Buckets mit der ersten Sortierung nach der $x$-Koordinatenrichtung. Auf der 
Abbildung 45 rechts und unten sind die Rekonstruktionen einerseits mit der globalen Methode und dem Euklidischen Hut mit hinreichend großem Trägerradius (jeweils oben) und andererseits mit der PoU Methode unter Verwendung des Volume Splines gezeigt.
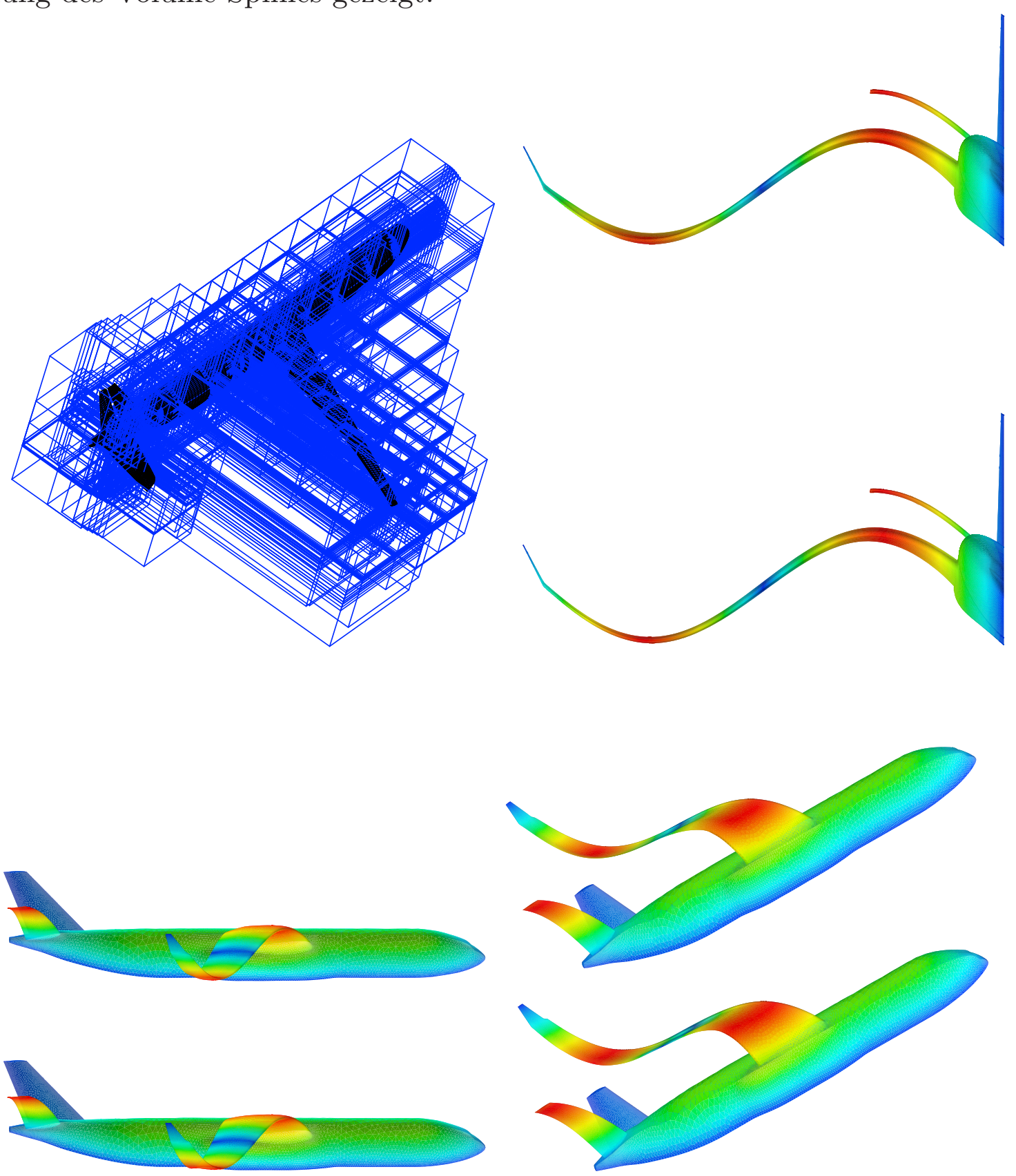

Abbildung 45. Links oben: Partitionen für den A340. Rechts und unten: Globale (oben) und PoU (unten) Rekonstruktion. 


\subsection{Das A340-300 Halbmodell - Ein realistischer Testfall}

In diesem Abschnitt werden weiterhin die Modelle des A340-300 des vorherigen Abschnitts benutzt. Allerdings soll jetzt eine realistischere Deformation betrachtet werden. Dazu wird eine quadratische Funktion entlang des Flügels in Richtung der Flugzeug-Höhe aufgetragen. Die an der Flügelspitze aufgebrachte Verschiebung, also die maximale Deformation, hat den Absolutwert 4.0 .

Da diese Deformation ähnlich einer realen Deformation ist, sind hier Fehlerrechnungen ausgeführt worden, indem die Differenz der interpolierten zu den exakten Werten auf den Strömungsknoten betrachtet wird. Die berechneten maximalen Fehler zeigt die Tabelle 9 für verschiedene radiale Basisfunktionen und unterschiedliche Anzahlen von polynomialen Termen. Dabei sind die notwendigen Terme nach Tabelle 4 zu berücksichtigen. Weitere Details über die genaue Parameterwahl finden sich wieder in der Tabelle $21 \mathrm{im}$ Anhang A.

Man beachte, dass bei der Verwendung quadratischer Polynome die Deformation unabhängig von der verwendeten Basisfunktion exakt reproduziert werden muss. Dies spiegelt sich darin wieder, dass in diesem Fall alle Basisfunktionen den gleichen kleinen Fehler liefern. Ohne quadratische Polynome liefert W3 die besten Ergebnisse, was an der Glätte der Zielfunktion liegt.

\begin{tabular}{|c|c|c|c|c|}
\hline RBF & ohne Pol. & Konstanten & lin. Pol. & quad. Pol \\
\hline TPS & - & - & $1.394994 e-06$ & $5.054963 e-08$ \\
\hline TPS4 & - & - & - & $5.054963 e-08$ \\
\hline VS & - & $1.124920 e-01$ & $3.440040 e-02$ & $5.054963 e-08$ \\
\hline VS3 & - & - & $6.530696 e-03$ & $5.054963 e-08$ \\
\hline EH & $1.050804 e-01$ & $1.124958 e-01$ & $3.438995 e-02$ & $5.054963 e-08$ \\
\hline W1 & $9.312255 e-02$ & $1.130730 e-01$ & $3.440040 e-02$ & $5.054963 e-08$ \\
\hline W2 & $6.350484 e-03$ & $6.237562 e-03$ & $6.582115 e-03$ & $5.054963 e-08$ \\
\hline W3 & $7.712456 e-04$ & $1.753188 e-03$ & $3.046383 e-02$ & $5.054963 e-08$ \\
\hline
\end{tabular}

Tabelle 9. Der Interpolationsfehler bei verschiedenen radialen Basisfunktionen und polynomialen Termen unterschiedlicher Ordnung.

Die Abbildung 46 zeigt exemplarisch die Ergebnisse der Interpolation unter Verwendung der Basisfunktion W3. Abbildung 47 zeigt links eine Vergrößerung des Flügels. Im rechten Teil dieser Abbildung ist der Interpolationsfehler auf den Flügel aufgetragen worden. Es ist zu erkennen, dass auch hier der größte Fehler an der Hinterkante des Flügels auftritt, wo keine Strukturinformation mehr vorliegt. 

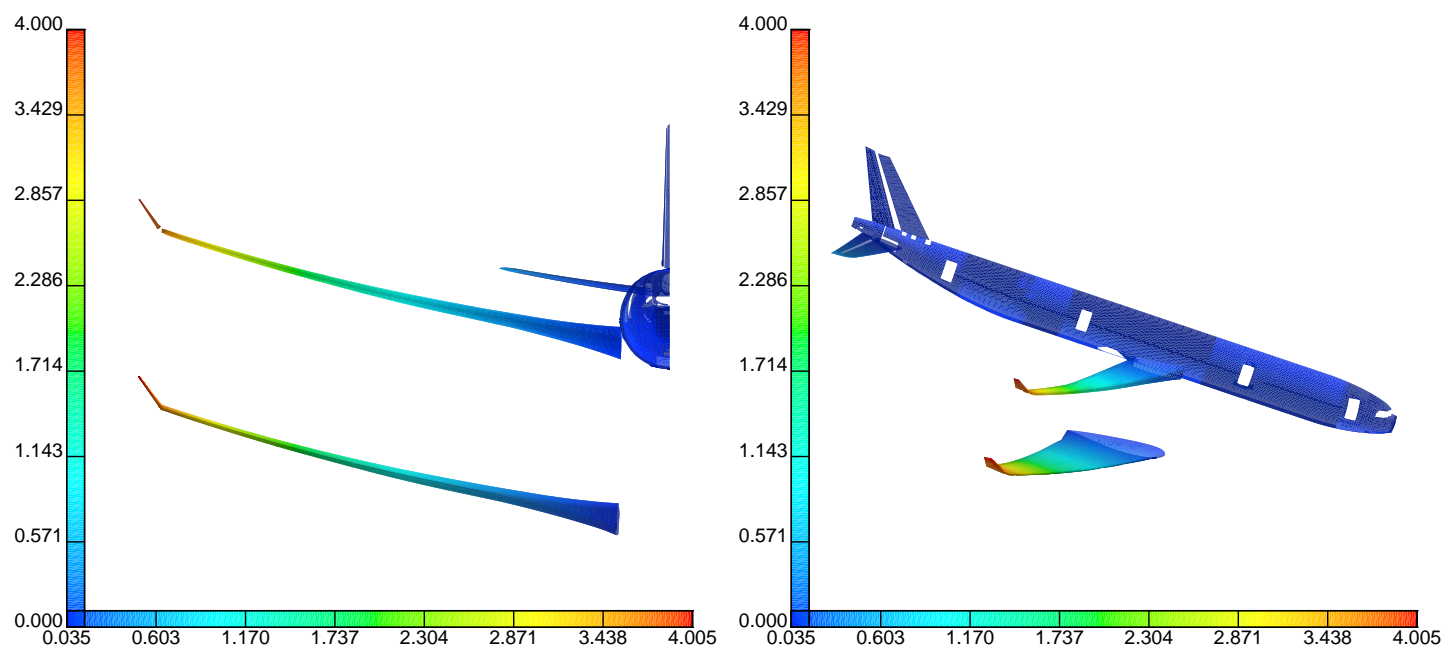

Abbildung 46. Deformation auf Strömungsseite mit W3 berechnet.
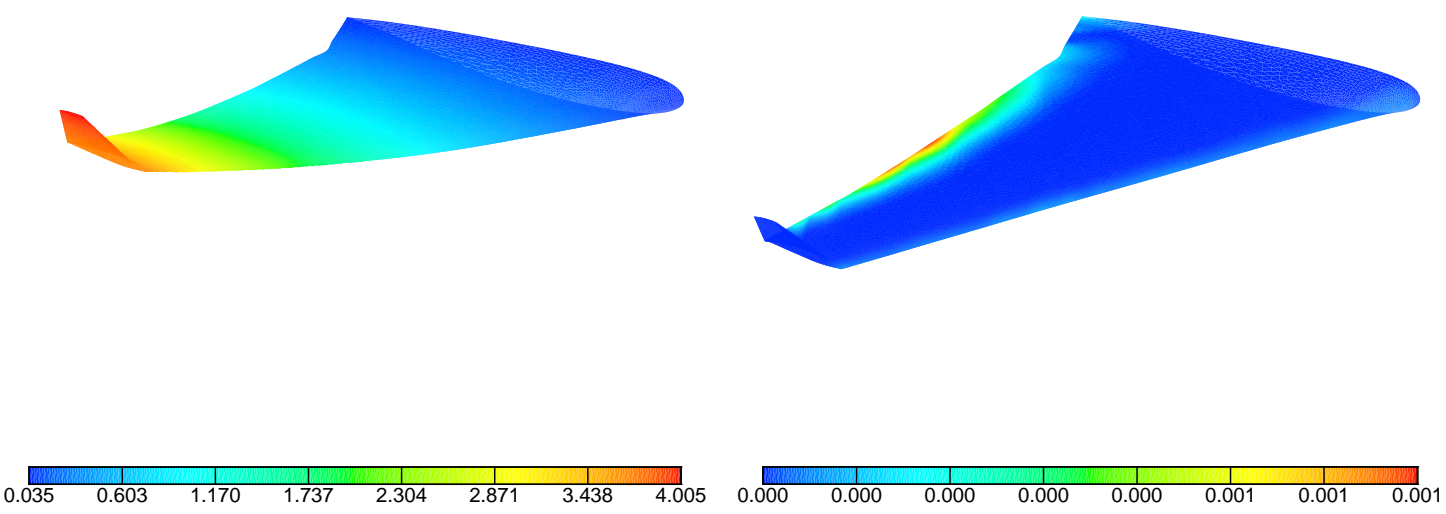

Abbildung 47. Deformation auf Strömungsseite mit W3 berechnet (links) und der zugehörige Fehler (rechts). 


\subsection{Datenaustausch in der Rohrleitung}

Das folgende Beispiel zeigt die Anwendung der PoU Methode zur Übertragung von Temperaturdaten von einem Rohr zu einem dicht daran liegenden Rohr mit Modellen unterschiedlicher Auflösung. Dieses Mapping von Temperaturen dient der Eigenspannungs-Berechnung von Rohrleitungen im Bereich Anlagenbau und Raumklimatisierung.

Das Rohr, auf dem die Temperaturen bestimmt werden sollen, liegt innerhalb des Rohrs mit den gegebenen Temperaturen. Das äußere Rohr mit den gegebenen Temperaturen ist wesentlich feiner aufgelöst. Also enthält bei dieser speziellen Anwendung die Stützstellenmenge wesentlich mehr Punkte als die Auswertungsmenge, siehe Tabelle 10. Ferner handelt es sich hier um ein "echtes" dreidimensionales Problem, d.h. es liegen auch Punkte im Inneren des Rohrs, so dass diesmal der Datensatz dichter an einen quasi-uniformen Datensatz heran kommt.

\begin{tabular}{|c|c|c|c|}
\hline $\begin{array}{c}\text { Anzahl } \\
\text { Stützstellen }\end{array}$ & $\begin{array}{c}\text { Anzahl } \\
\text { Auswertungsstellen }\end{array}$ & $h_{X, \Omega}$ & $q_{X}$ \\
\hline 24782 & 4316 & $2.664947 e-03$ & $4.341212 e-04$ \\
\hline
\end{tabular}

Tabelle 10. Die Modellgrößen der Anwendung.

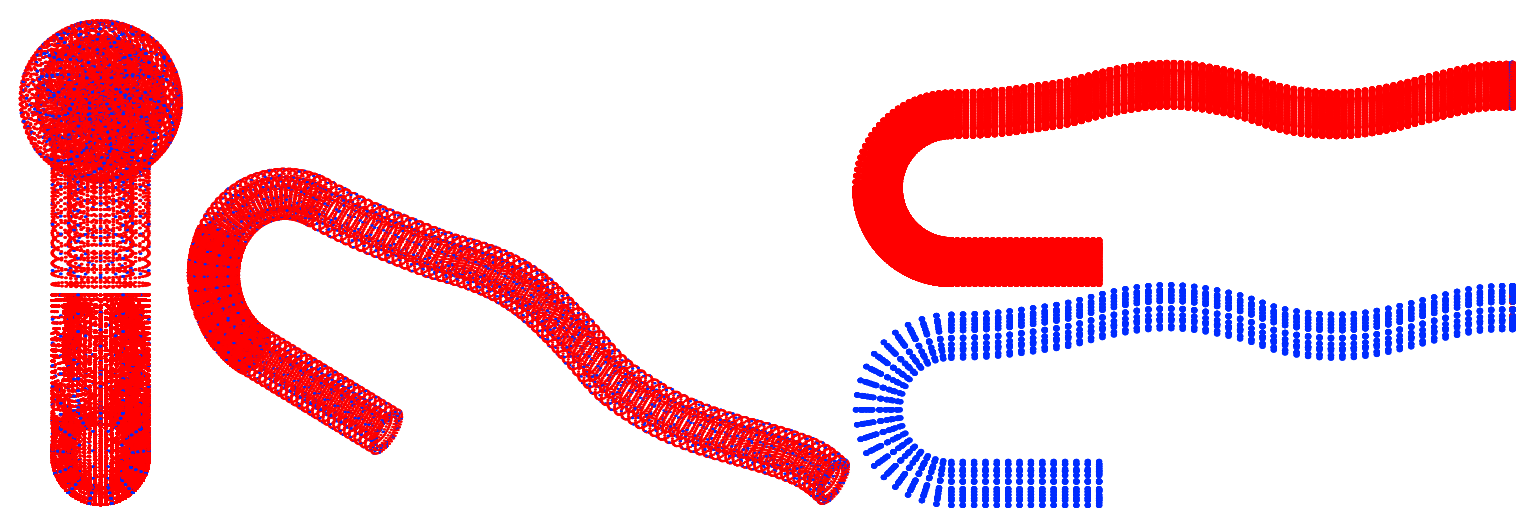

Abbildung 48. Die beiden Rohrmodelle zusammen (links u. Mitte) und getrennt (rechts).

In Abbildung 48 sind die Knoten der beiden Rohrmodelle dargestellt, zunächst in der Lage zueinander (links und Mitte) und dann zur besseren Darstellung wieder mit einem vertikalen Abstand (rechts). Für die Visualisierung werden bei diesem Beispiel nur die Knoten gezeigt. Die Knoten des äußeren Rohrs mit den gegebenen Temperaturen sind als rote Punkte dargestellt und die Knoten des inneren Rohrs sind als blaue Punkte gezeigt. Die Abbildungen in der Mitte und rechts zeigen die Rohre von der Seite. Bei der Abbildung links wird in die Rohröffnungen gesehen.

Die gegebenen Temperaturen auf dem ersten Rohr und die interpolierten Temperaturen auf dem zweiten Rohr sind in den Abbildungen 49 bis 54 visualisiert. Die Temperaturen sind farblich dargestellt. 
Die Berechnungsverfahren und Parameter zu den Abbildungen sind, wie schon in allen vorherigen Anwendungsbeispielen, in der zugehörigen Tabelle 22 im Anhang A aufgelistet.

Die gegebenen Temperaturen sind über Simulationscodes berechnet, sodass hier keine Abweichungen zu gegebenen Funktionen angegeben werden können. Die Interpolation über die globale Anwendung der radialen Basisfunktionen ist auf Grund der Größe des Beispiels nicht mehr möglich. Da auch keine Messwerte zum Vergleich existieren bleibt nur die Überprüfung durch die Visualisierung. Wie in den Abbildungen 49 bis $54 \mathrm{zu}$ sehen ist, zeigen sich auch bei dieser Anwendung sinnvolle Resultate bei der Interpolation über die „Partition of Unity“ Methode.

In allen Abbildungen mit Legende bezieht sich die vertikale Legende auf die gegebene Temperatur und die horizontale Legende auf die interpolierte Temperatur. Dem Interpolationsverfahren liegt eine PoU mit $14 \cdot 14 \cdot 15$ Buckets zu Grunde. Die verwendete Basisfunktion ist VS.

In den Abbildungen 49 und 50 sind verschiedene Ansichten der beiden Rohre zu sehen. Die gegebenen Temperaturen sind jeweils auf dem oberen Rohr zu sehen, während das untere Rohr die interpolierten Werte enthält. Die Knoten mit der farblichen Darstellung der Werte sind in den Abbildungen mit unterschiedlich großer Ausdehnung gezeigt, um verschiedene Strukturen in den Temperaturverläufen sichtbar zu machen.
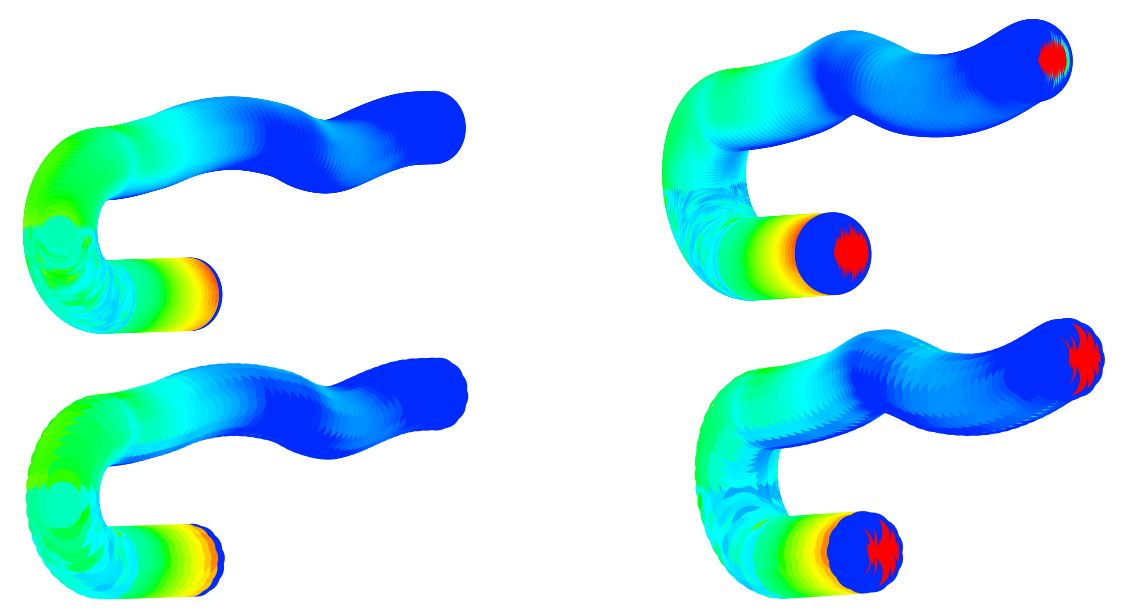

Abbildung 49. Die gegebenen (oberes Rohr) und die interpolierten (unteres Rohr) Temperaturen. Die Interpolation wurde mit der PoU Methode unter Verwendung von VS durchgeführt.

In den Abbildungen 51 und 52 sind Schnitte durch beide Rohre gelegt worden, die wieder entsprechend ihrer Temperatur eingefärbt wurden. Dabei ist auf der linken Seite jeweils der Schnitt durch das äußere Rohr (links) und das innere Rohr (rechts) zu sehen. Es ist deutlich zu erkennen, dass die Modelle unterschiedliche Auflösung haben. Im rechten Teil der Abbildungen ist die Lage der Schnitte im Rohr gezeigt.

Die Abbildungen 53 und 54 zeigen ebenfalls Schnitte, allerdings ist jetzt die gegebene Temperatur oben und die interpolierte unten gegeben. 

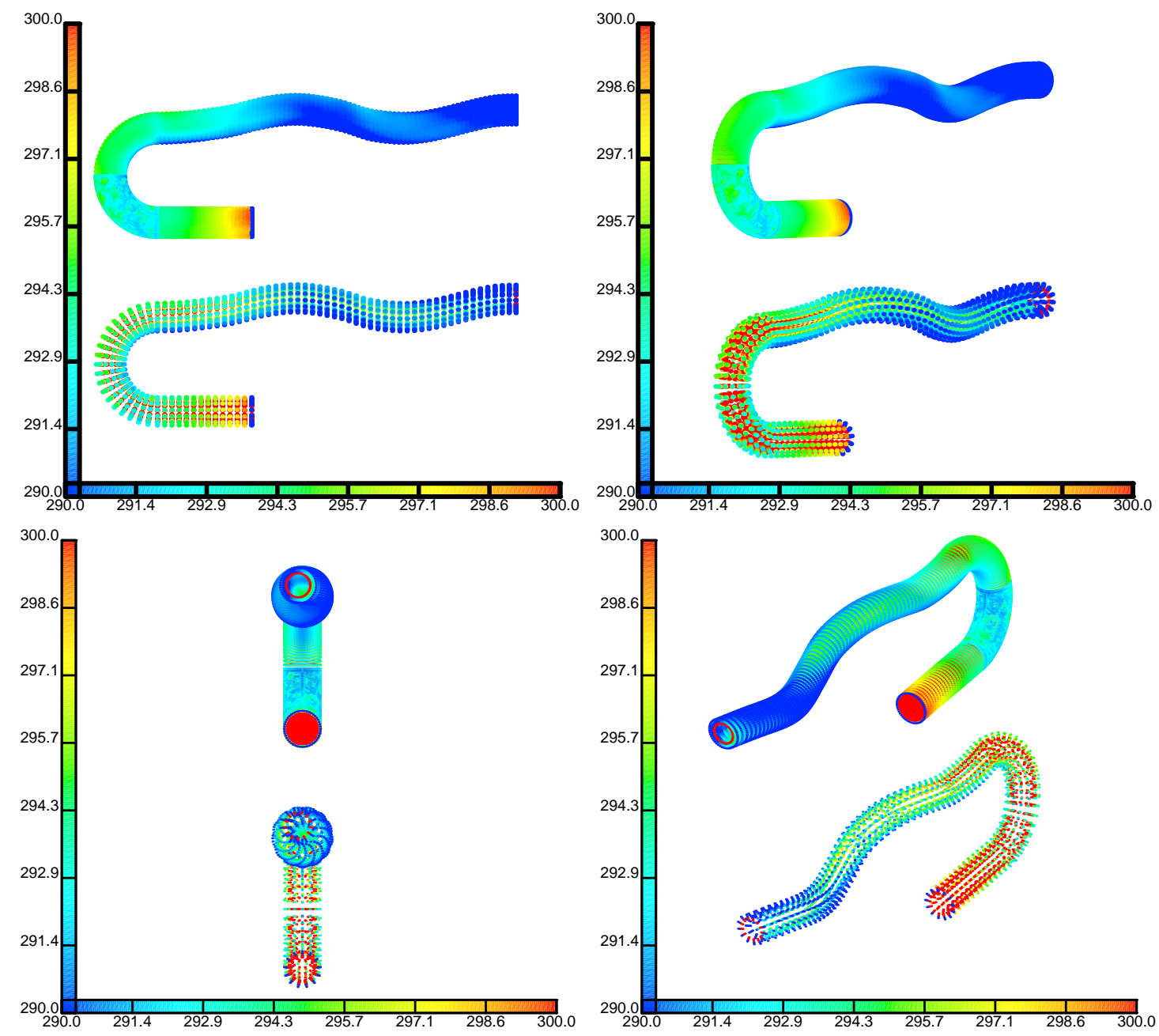

Abbildung 50. Die gegebenen (oberes Rohr) und die interpolierten (unteres Rohr) Temperaturen. Die Interpolation wurde mit der PoU Methode unter Verwendung von VS durchgeführt.
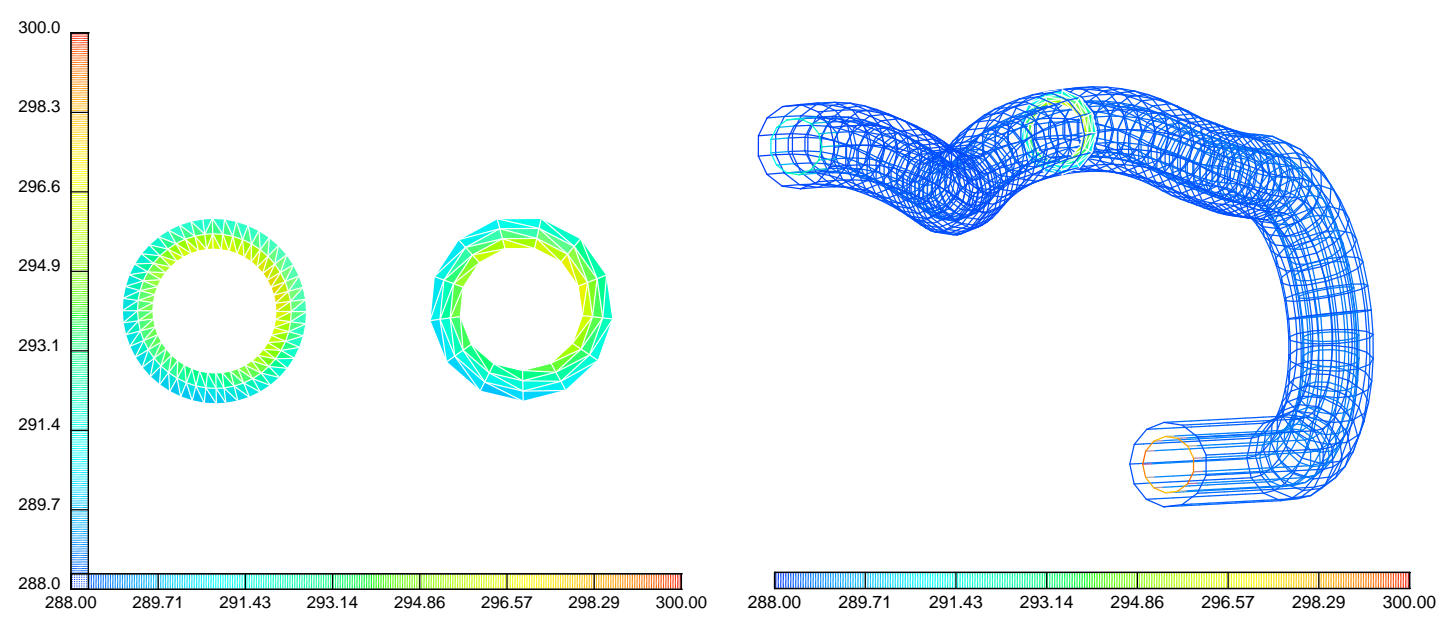

Abbildung 51. Gegebene und interpolierte Temperaturverteilung auf Schnitten durch die Rohre (links) und deren Lage im jeweiligen Rohr (rechts). Die Interpolation wurde mit der PoU Methode unter Verwendung von VS durchgeführt. 

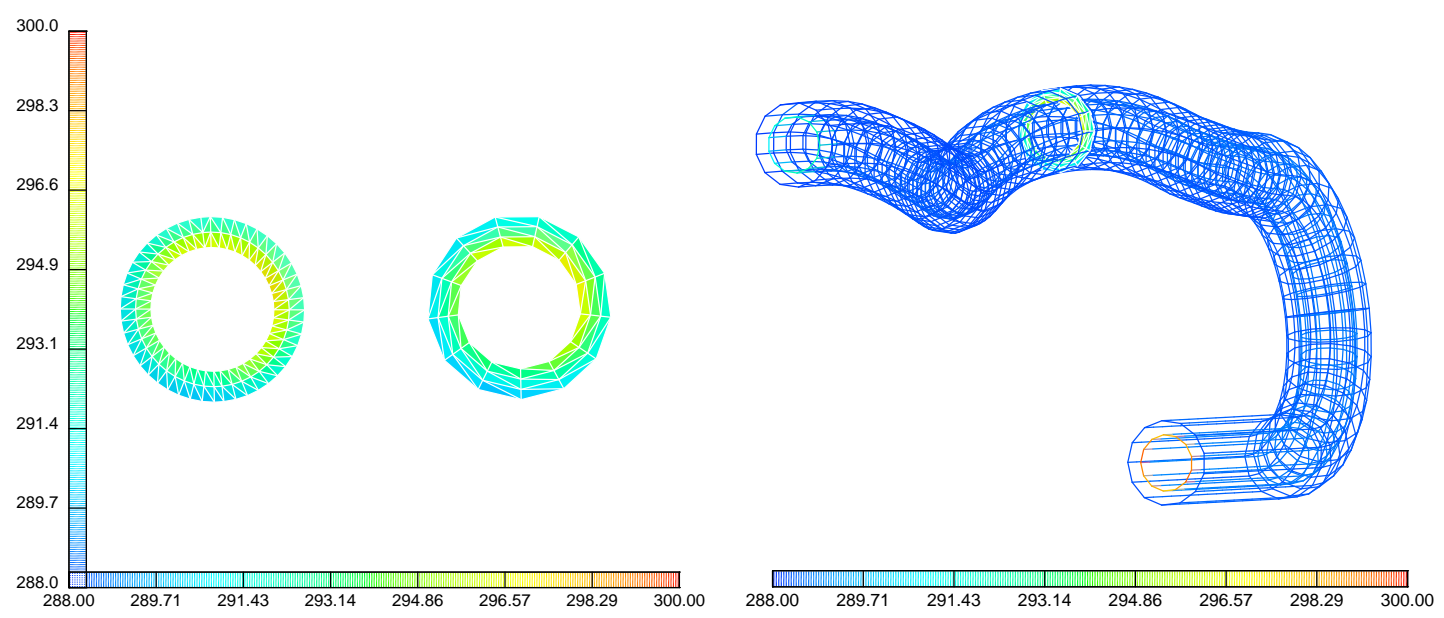

Abbildung 52. Gegebene und interpolierte Temperaturverteilung auf Schnitten durch die Rohre (links) und deren Lage im jeweiligen Rohr (rechts). Die Interpolation wurde mit der PoU Methode unter Verwendung von VS durchgeführt.
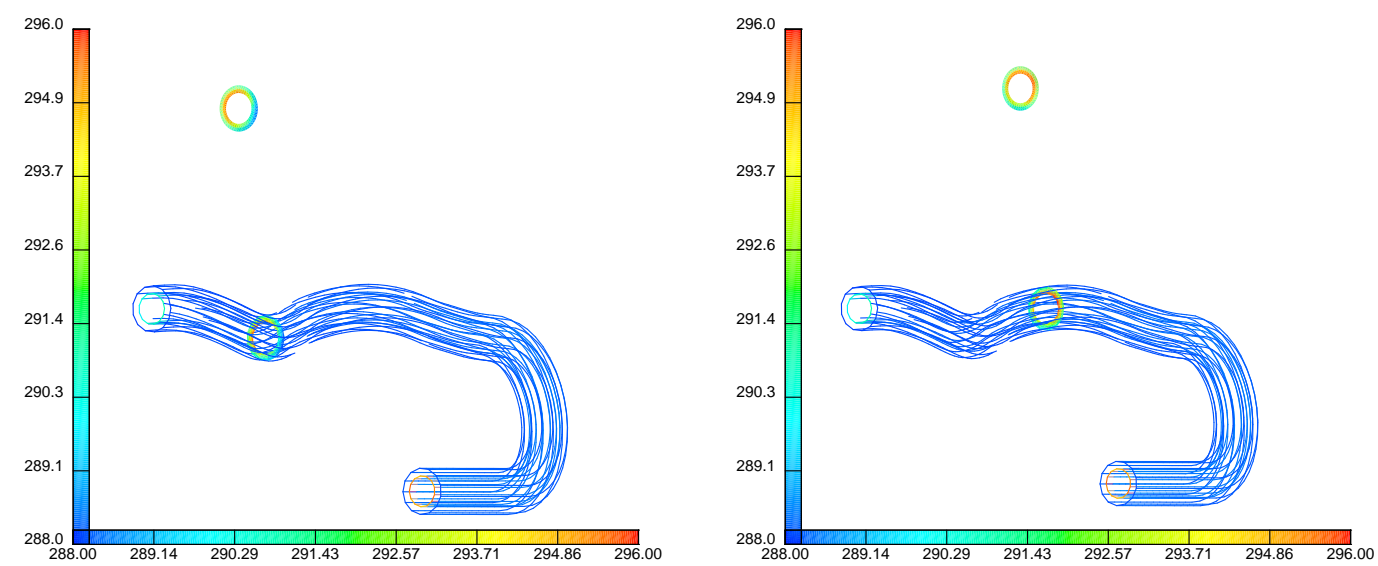

Abbildung 53. Gegebene (oben) und interpolierte (unten, im Rohr gezeigt) Temperaturverteilung auf Schnitten durch die Rohre. Die Interpolation wurde mit der PoU Methode unter Verwendung von VS durchgeführt.
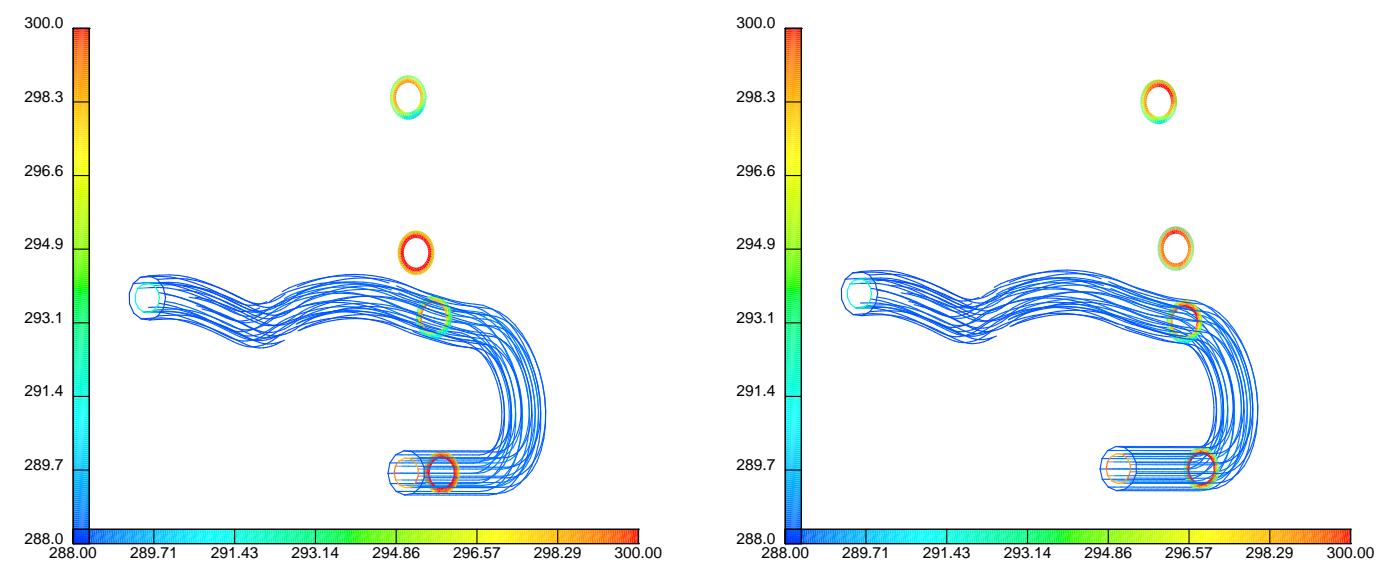

Abbildung 54. Gegebene (oben) und interpolierte (unten, im Rohr gezeigt) Temperaturverteilung auf Schnitten durch die Rohre. Die Interpolation wurde mit der PoU Methode unter Verwendung von VS durchgeführt. 


\subsection{Datenaustausch in der Sprühlackierung}

Die Interpolationsbeispiele aus dem Bereich der Sprühlackierung sollen eine weitere Anwendung der „Partition of Unity“ Methode demonstrieren. Die Simulation der Sprühlackierung mit elektrostatischer Außenaufladung ist ein gekoppeltes elektro-hydrodynamisches Problem, bei dem Wechselwirkungen zwischen einer dispersen 2-Phasenströmung mit Ionenanteil und einem von Elektrodenkonfiguration und räumlicher Ladungsdichteverteilung - aufgrund von Ionen und geladenen Partikeln - erzeugten elektrischen Feld bestehen. Zwischen den Phasen findet ein Austausch von Impuls, Masse, Energie und Ladung statt.

Die Feldgleichungen für Strömung, Zwangskonvektion und elektrisches Feld werden auf problemangepassten individuellen Gittern gelöst, so dass eine Volumen-Volumen-Interpolation zwischen unstrukturierten Netzen überlappender Gebiete zum Austausch der Daten benötigt wird.

Die Berechnung der Partikeltrajektorien erfolgt ohne Gitter. Zur Übernahme von Zustandswerten der kontinuierlichen Strömung, der Ionenkonzentration und der elektrischen Feldstärke wird ein gleichmäßiges Voxelgitter vorausgesetzt, und in gleicher Weise werden Ergebnisse der dispersen Phase (Volumenanteil, Impulsquellterme, Ladungsdichte, etc.) in einem solchen Voxelgitter abgelegt. Daher wird auch hierfür eine Volumen-Volumen-Interpolation benötigt. Die Interpolation soll die Erhaltung physikalisch relevanter Größen wie Masse, Ladung, Impuls, und elektrische Feldenergie gewährleisten.

Die zweidimensionalen Beispiele zeigen über diskrete Modelle berechnete Felder, an denen die Interpolationen getestet werden sollten. Das erste Beispiel zeigt das elektrostatische Potential bei der Sprühlackierung für eine axialsymmetrische Anordnung. Die drei weiteren Testfelder sind die drei Komponenten des Geschwindigkeitsvektors in Zylinderkoordinaten ebenfalls für eine axialsymmetrische Anordnung. Interpoliert wird von den problemangepassten Gittern auf regelmäßige Rechteck-Gitter mit unterschiedlicher Auflösung.

Die Abbildung 55 zeigt das verwendete Ausgangsgitter mit den Stützstellen. Die Auswertungspunkte stellen die Knoten eines gleichmäßigen $200 \times 200$ Rechteckgitters dar. Der Bereich des Auswertungsstellengitters ist in Abbildung 55 durch eine blaue Fläche gekennzeichnet.

Auf der Abbildung 56 ist die Partitionierung des Stützstellengitters gezeigt. Die Stützstellen sind in $120 \cdot 121$ überlappende Buckets aufgeteilt. In der Abbildung der Partitionierung ist auf der linken Seite die Partitionierung angefangen mit der Sortierung nach der x-Koordinatenrichtung und auf der rechten Seite die Partitionierung angefangen mit der Sortierung nach der y-Koordinatenrichtung dargestellt. In der Abbildung 58 sind die gegebenen Potentialwerte der Stützstellen (links) gezeigt und die interpolierten Potentialwerte auf den Auswertungsstellen (rechts), berechnet über die „Partition of Unity" Methode mit der in der Abbildung 56 dargestellten Aufteilung in $120 \cdot 121$ Buckets. Die verwendete radiale Basisfunktion ist die Wendland 2 


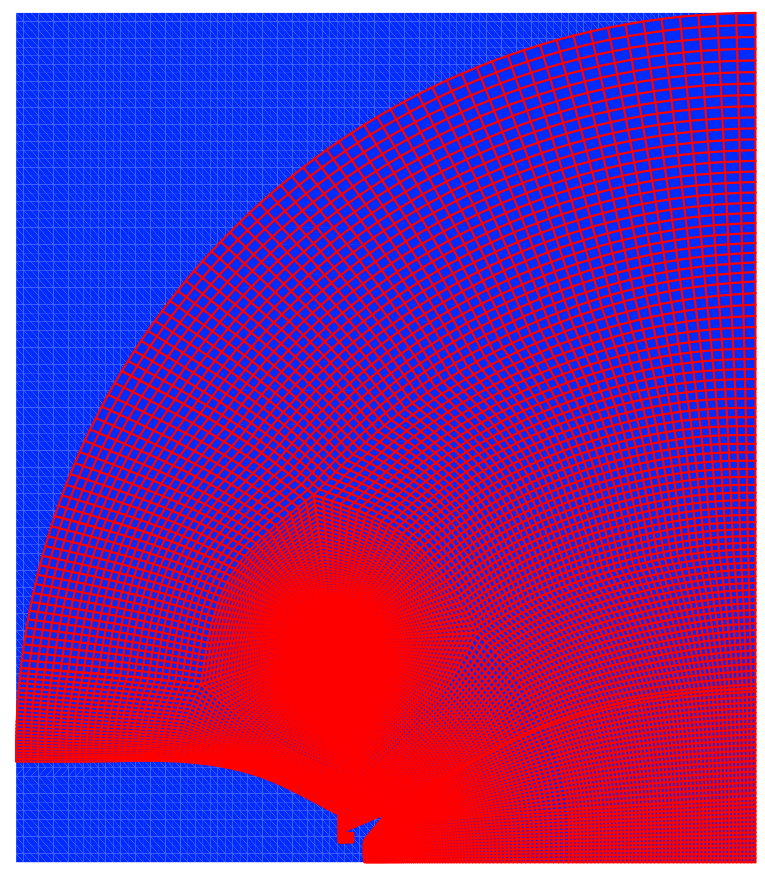

Abbildung 55. Das Stützstellengitter (rot) und der Auswertungsbereich (blau).

Funktion. Der Wert des elektrostatischen Potentials wird farblich dargestellt. Die vertikale Legende bezieht sich in allen Abbildungen dieses Abschnitts auf die gegebenen Potentialwerte und die horizontale auf die interpolierten Potentialwerte.

In der Abbildung 57 ist der Wert des elektrostatischen Potentials durch Verschiebung der Gitterpunkte in Richtung der Normalen der zweidimensionalen Gitterfläche dargestellt. Die Fläche wird in der Mitte und unten aus verschiedenen Perspektiven gezeigt, so dass alle Seiten betrachtet werden können. Der Wert des elektrostatischen Potentials wird zusätzlich farblich dargestellt. Die Ausgangswerte und die interpolierten Werte sind die gleichen wie in Abbildung 58. Jeweils links in den mittleren Abbildungen und rechts in den unteren Abbildungen sind die gegebenen Potentialwerte der Stützstellen dargestellt und jeweils daneben sind die interpolierten Potentialwerte auf den Auswertungsstellen gezeigt und ermöglichen so einen direkten Vergleich. In Abbildung 57 oben werden die beiden in der selben Abbildung gezeigten Flächen mit den Potentialwerten der Stützstellen und den interpolierten Potentialwerten übereinander gelegt, um zu zeigen, dass keine Abweichungen der beiden Flächen voneinander zu erkennen sind.

Weiterhin sind in Abbildung 59 oben und unten die Werte der gegebenen Geschwindigkeitskomponenten (Zylinderkoordinaten) und interpolierten Geschwindigkeitskomponenten dargestellt. Die Geschwindigkeitskomponente in $r$-Richtung, in $\phi$-Richtung und in $z$-Richtung sind von oben bis unten rechts in der aufgezählten Reichenfolge gezeigt. Der Wert der einzelnen Geschwindigkeitskomponenten wird farblich dargestellt. Links sind jeweils die Werte der Geschwindigkeitskomponenten der Stützstellen und rechts die interpo- 

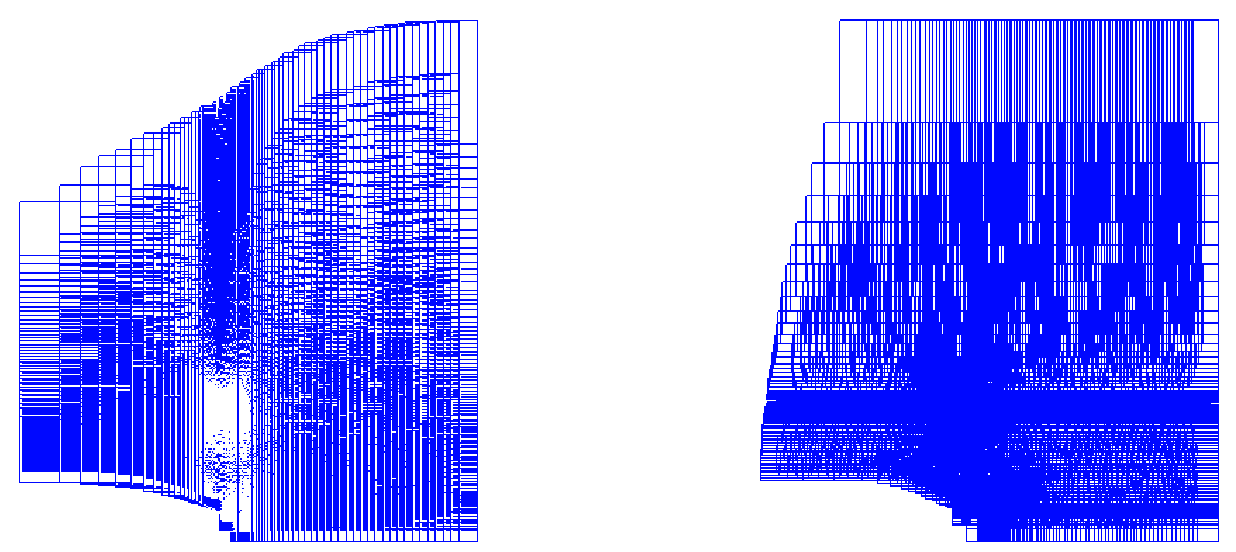

Abbildung 56. Die Partitionen des Stützstellengitters.

lierten Werte der Geschwindigkeitskomponenten auf den Auswertungsstellen zu sehen. Die Interpolation ist wieder über die „Partition of Unity“ Methode mit der in Abbildung 56 gezeigten Aufteilung in $120 \cdot 121$ Buckets berechnet worden. Als radiale Basisfunktion wurde hier die Wendland 1 Funktion eingesetzt.

Bei diesen Anwendungen sind in der Tabelle 11 die in Abschnitt 2.3 beschriebenen Spezifikationen zu den Modellgrößen enthalten. Die Berechnungsverfahren und Parameter zu den Abbildungen sind in der Tabelle 22 im Anhang A zu finden.

\begin{tabular}{|c|c|c|c|}
\hline $\begin{array}{c}\text { Anzahl } \\
\text { Stützstellen }\end{array}$ & $\begin{array}{c}\text { Anzahl } \\
\text { Auswertungsstellen }\end{array}$ & $h_{X, \Omega}$ & $q_{X}$ \\
\hline 30279 & 40000 & $1.670616 e-01$ & $1.427432 e-05$ \\
\hline
\end{tabular}

Tabelle 11. Die Modellgrößen der Anwendung.

Wie im vorherigen Abschnitt 4.3 werden wieder über diskrete Modelle berechnete Verteilungen interpoliert und es liegen keine Messwerte für Vergleichsrechnungen vor. Da die globale Anwendung der radialen Basisfunktionen zur Interpolation auf Grund der Größe des Beispiels nicht mehr möglich ist, bleibt auch hier nur die Überprüfung mittels der Visualisierung. Es zeigt sich auch bei diesen Anwendungen der „Partition of Unity" Methode beste Übereinstimmung, zudem konnte mit der Methode der gewünschte Effekt erreicht werden, dass die Werte in den Extrapolationsbereichen also außerhalb des Stützstellengitters auf Null abfallen. Dieser Effekt ist gewünscht, da in den Extrapolationsbereichen auf Grund von Randbedingungen keine Felder vorhanden sein sollen. 

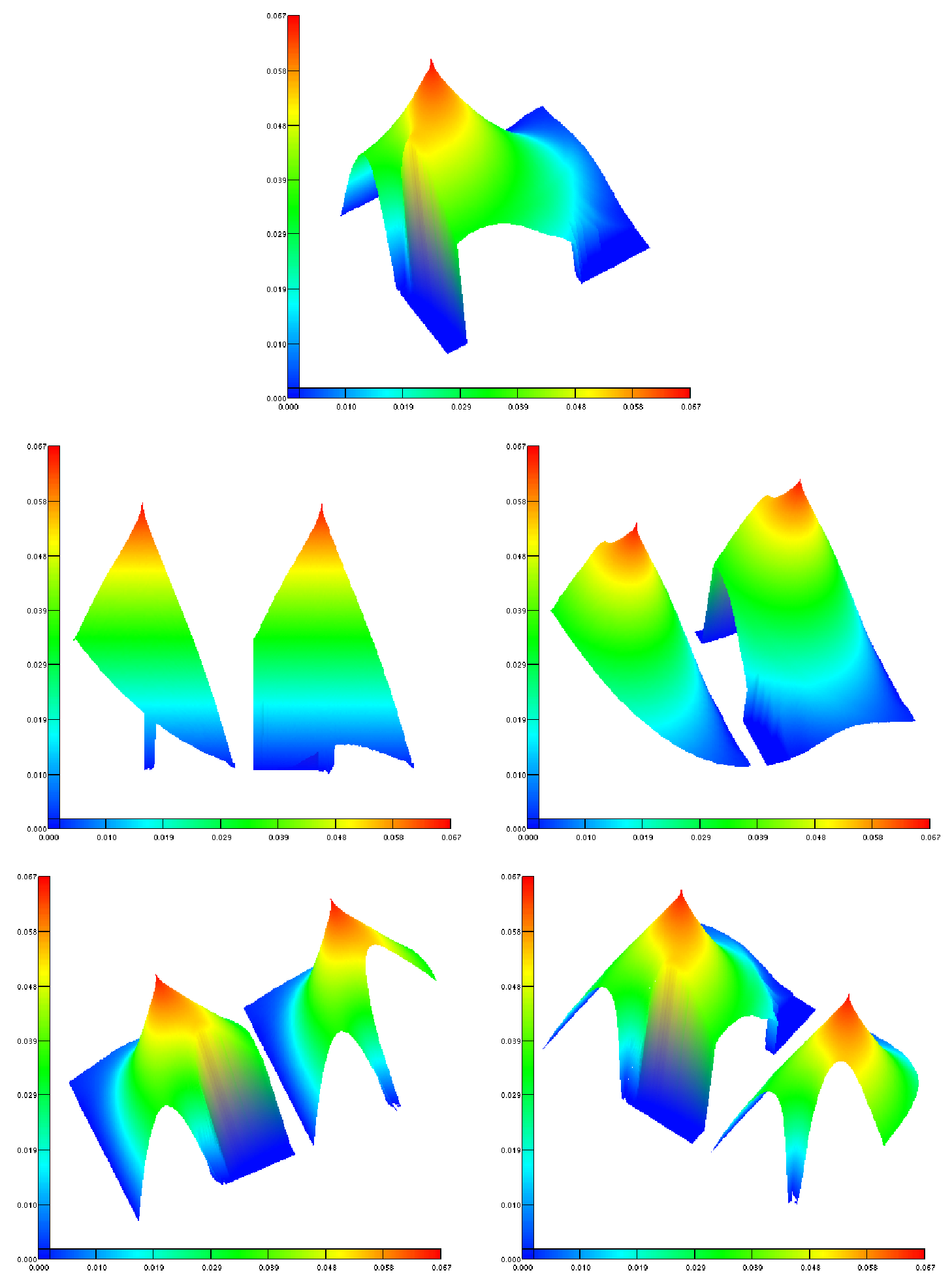

Abbildung 57. Die gegebenen und interpolierten Potentialwerte nebeneinander räumlich dargestellt. Die Interpolation wurde mit der PoU Methode unter Verwendung von W2 durchgeführt. 


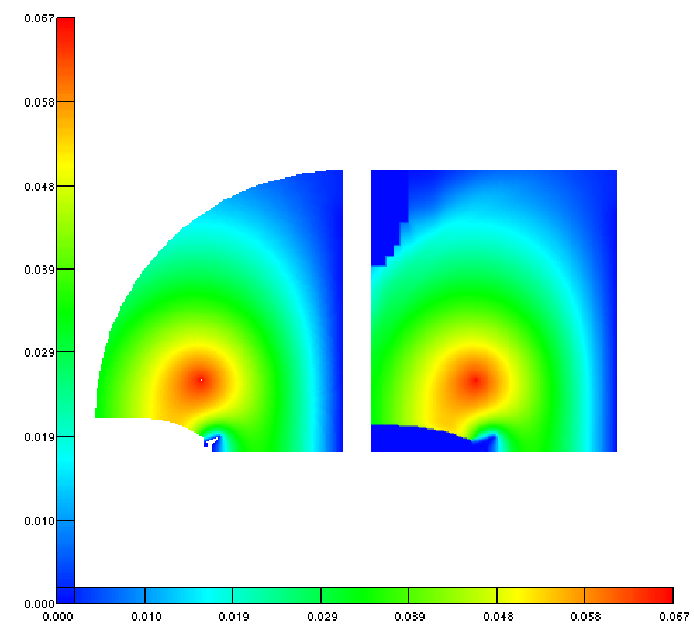

Abbildung 58. Die gegebenen (links) und interpolierten (rechts) Potentialwerte. Die Interpolation wurde mit der PoU Methode unter Verwendung von W2 durchgeführt.
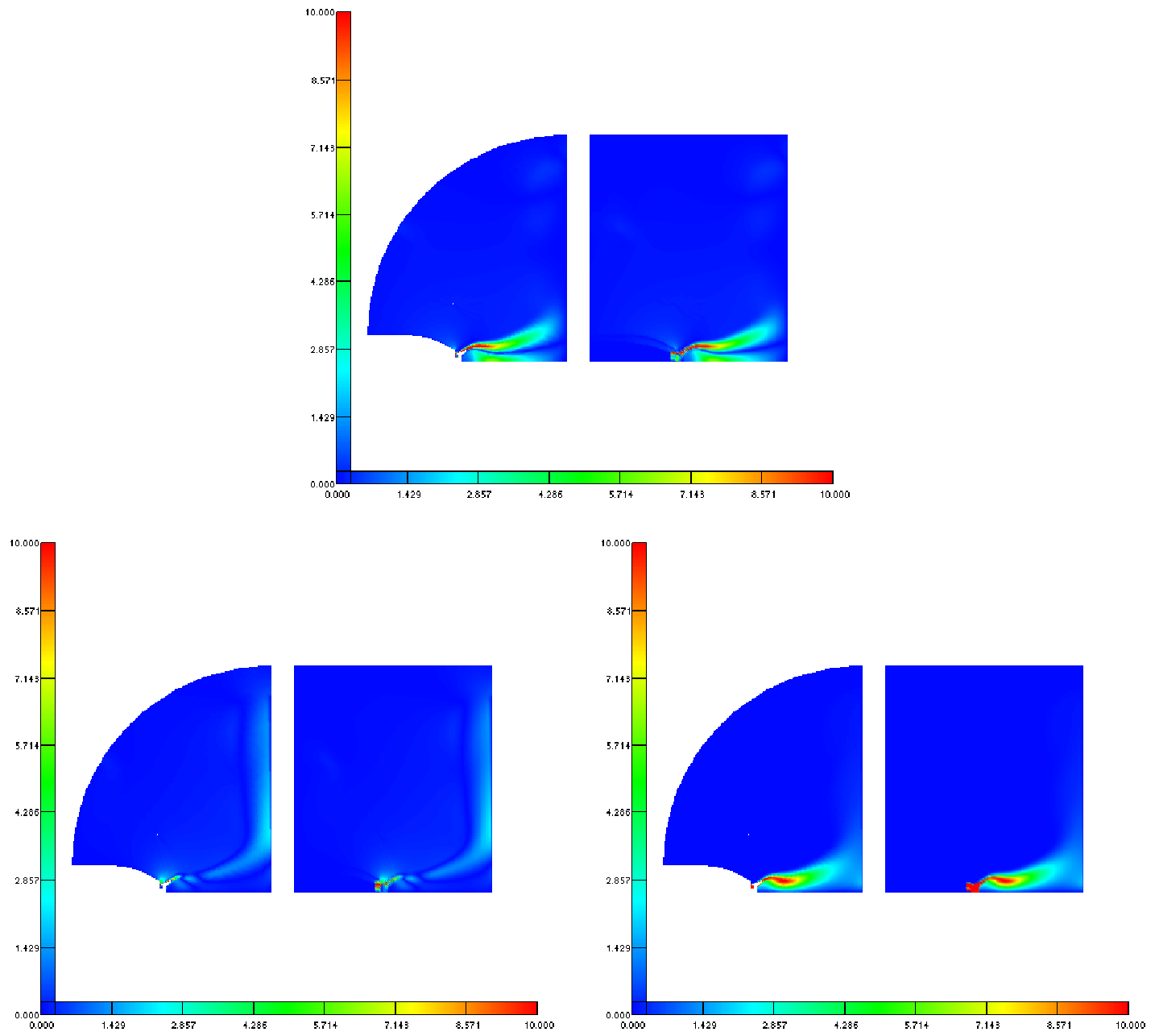

Abbildung 59. Die gegebenen und interpolierten Geschwindigkeitskomponenten. Die Interpolation wurde mit der PoU Methode unter Verwendung von W1 durchgeführt. 


\subsection{Datenaustausch in der Umformtechnik}

Ein weiteres Beispiel zur Demonstration der „Partition of Unity“ Methode stammt aus dem Bereich der Umformtechnik. Hier werden über die Interpolation Spannungswerte von einem Blechmodell auf ein anliegendes kleineres Blechmodell übertragen.
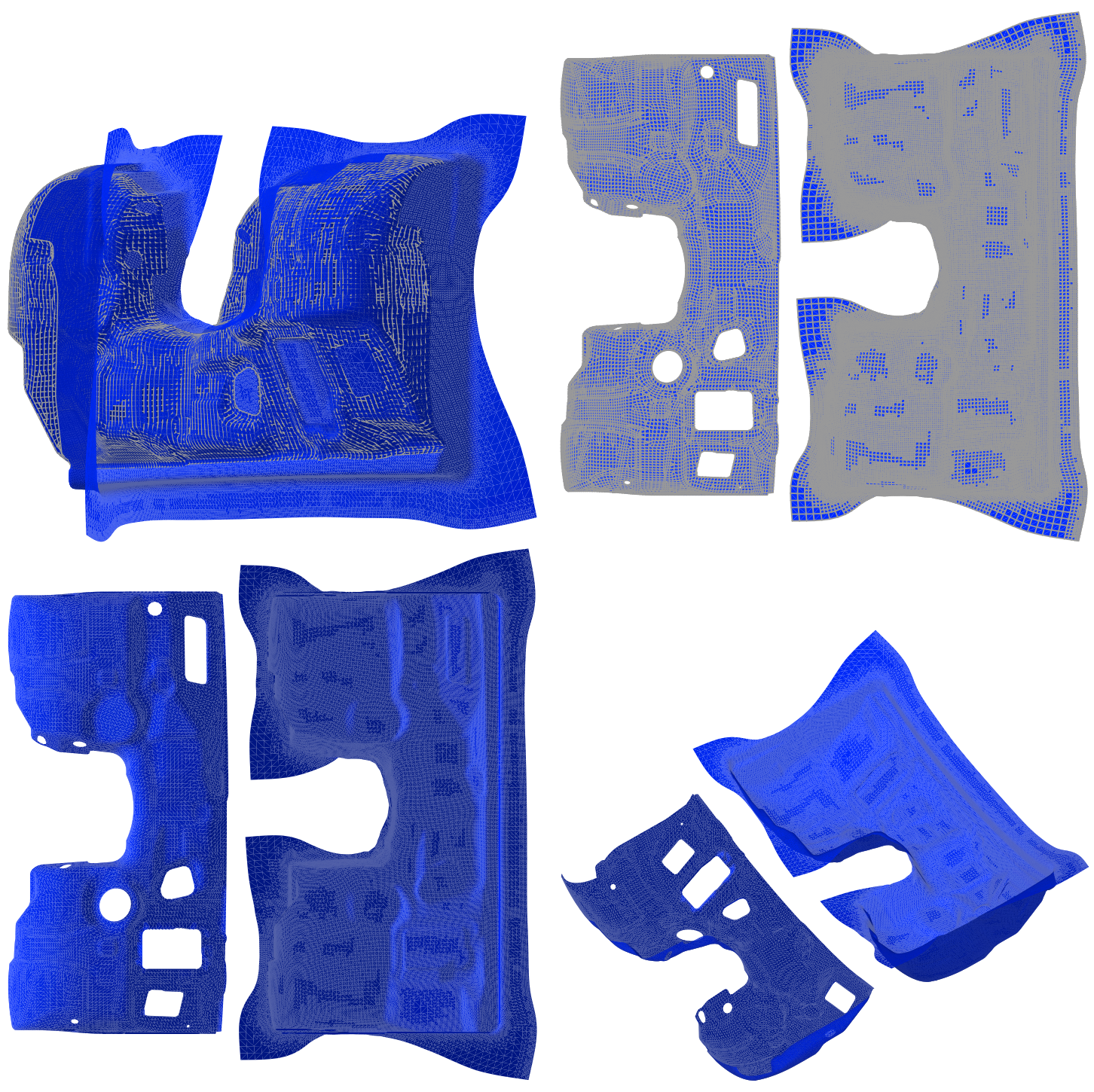

Abbildung 60. Die beiden Blechmodelle zusammen (oben links) und getrennt (oben rechts und unten).

Die Abbildung 60 zeigt die Oberflächen und die Gitter der beiden Blechmodelle aus verschiedenen Perspektiven, in der Lage zueinander und zur besseren Darstellung mit einem horizontalen Abstand zueinander. Auf der Abbildung links oben ist das Gitter des kleineren Blechmodells und nur die Oberfläche des größeren Blechs dargestellt, um die Lage des kleineren Blechmodells an dem größeren Blechmodell sichtbar zu machen. Die Abbildungen 
rechts oben und unten stellen die beiden Blechmodelle mit einem horizontalen Abstand getrennt voneinander dar. Oben sind die Gitter gezeigt und unten nur die Oberflächen der Blechmodelle aus verschiedenen Perspektiven. Die Abbildung links unten zeigt die Blechwölbungen von unten, bei der Abbildung rechts unten wird in die Wölbungen hineingesehen.

Die gegebenen Spannungen auf dem größeren Blech und die interpolierten Spannungen auf dem kleineren Blech sind in der Abbildung 61 dargestellt. Die Spannungen sind farblich visualisiert. Die vertikale Legende bezieht sich auf die gegebenen Spannungen und die horizontale auf die interpolierten Spannungen. Die Interpolation ist über die „Partition of Unity“ Methode mit einer Aufteilung in 29 $29 \cdot 29$ Buckets berechnet worden. Als radiale Basisfunktion wurde die Wendland 3 Funktion verwendet. Die Abbildungen oben und in der Mitte zeigen die Blechwölbungen aus verschiedenen Perspektiven. Die Abbildungen unten zeigen Ausschnitte der Bleche mit unterschiedlichen Farbskalierungen.

Die in Abschnitt 2.3 beschriebenen Spezifikationen zu den Modellgrößen sind in der Tabelle 12 aufgelistet, wie in allen vorherigen Anwendungsbeispielen. In der Tabelle $22 \mathrm{im}$ Anhang A sind auch die Abbildungen dieser Anwendung mit den Spezifikationen zu den Berechnungsalgorithmen aufgeführt.

\begin{tabular}{|c|c|c|c|}
\hline $\begin{array}{c}\text { Anzahl } \\
\text { Stützstellen }\end{array}$ & $\begin{array}{c}\text { Anzahl } \\
\text { Auswertungsstellen }\end{array}$ & $h_{X, \Omega}$ & $q_{X}$ \\
\hline 50434 & 10759 & $1.311965 e+01$ & $3.055616 e-01$ \\
\hline
\end{tabular}

Tabelle 12. Die Modellgrößen der Anwendung.

Durch die Größe des Beispiels kann wieder kein Vergleich zur Interpolation mit globaler Anwendung der radialen Basisfunktionen erfolgen. Da auch die hier gegebenen Spannungen aus einer Berechnung mit einem diskreten Modell stammen und keine Messwerte existieren, können keine Abweichungen zu einer gegebenen Funktion oder zu Daten aus einer Messung angegeben werden. So bleibt wieder nur die Überprüfung durch die Visualisierung; diese zeigt wieder sinnvolle Resultate auch für diesen Bereich der Anwendung der Interpolation über die „Partition of Unity“ Methode. 

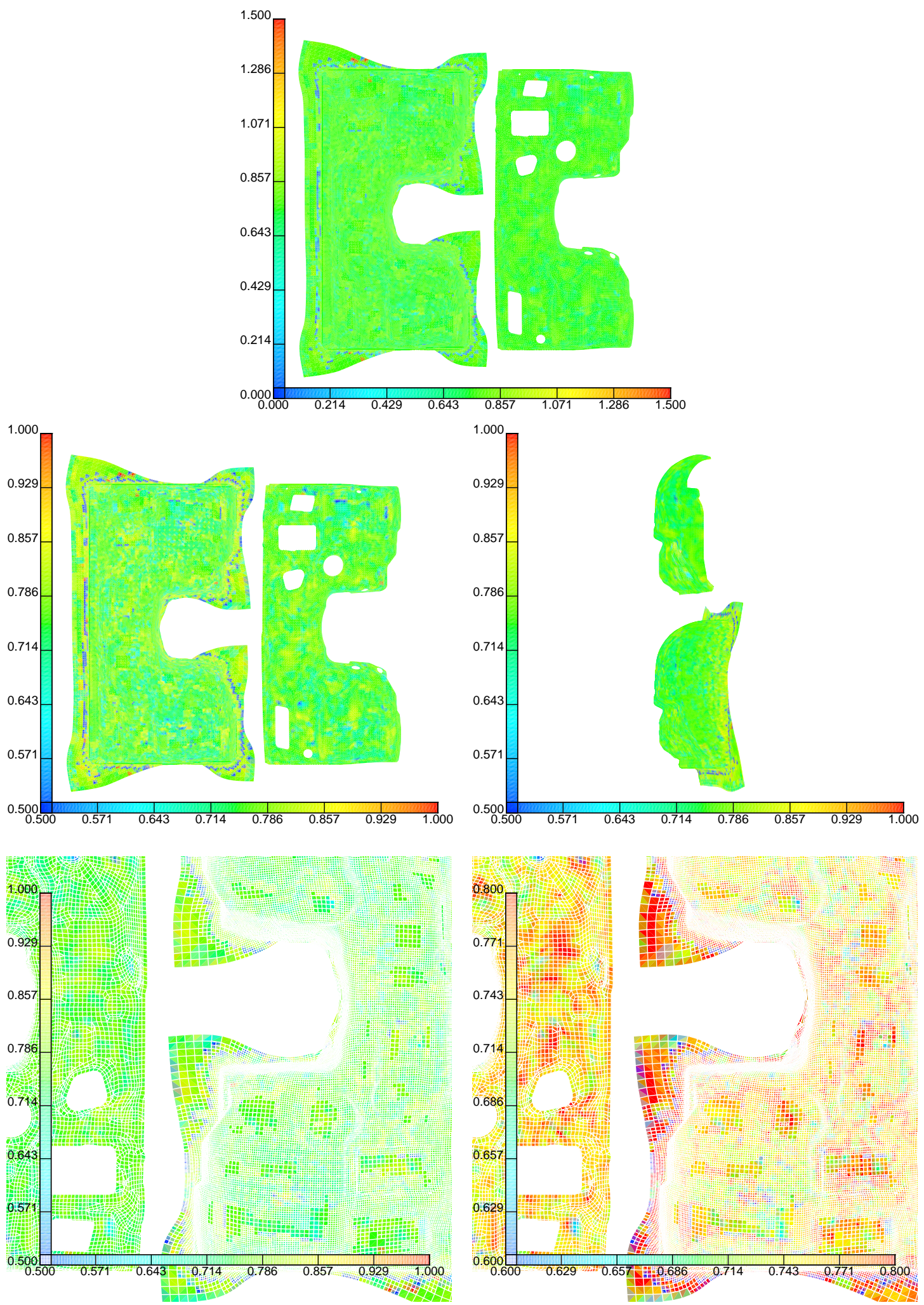

Abbildung 61. Die gegebenen und interpolierten Spannungen. Die Interpolation wurde mit der PoU Methode unter Verwendung von W3 durchgeführt. 


\section{Anwendungen des „Partition of Unity“ Verfahrens unter Einbeziehung von Arbeitserhaltung und Konservativität}

\subsection{Strömung-Struktur-Kopplung am ALENIA Halbmodell}

In diesem Abschnitt wird die Anwendbarkeit der „Partition of Unity“ Methode mit Zusatzbedingungen zur Erhaltung der Arbeit und zur Konservativität in der Interpolation auf ein aeroelastisches Testbeispiel gezeigt. Es wird ein Vergleich gezogen zur Transformation über die Transponierte der mit der „Partition of Unity“ Methode erstellten Kopplungsmatrix.
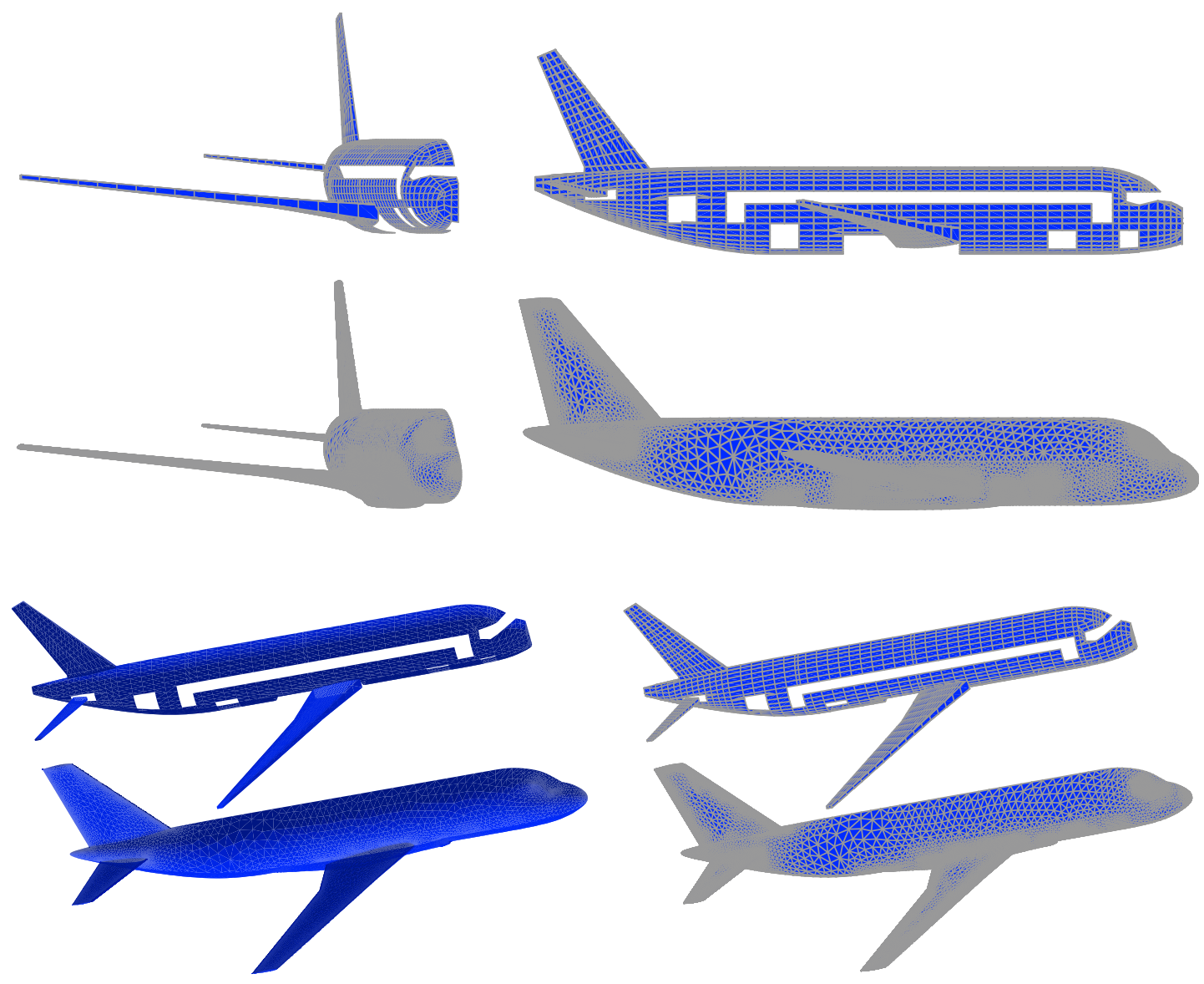

Abbildung 62. Das Strömungsgitter (unten) und Strukturgitter (oben) des ALENIA Halbmodells.

In Abbildung 62 sind das Struktur- und Strömungsgitter gemeinsam dargestellt. Die Struktur- und die Strömungsseite werden wieder durch ein Halbmodell beschrieben. Die Oberflächen bzw. Gitter sind zur besseren Darstellung auf den Abbildungen mit einem vertikalen Abstand voneinander gezeigt. Das Strukturgitter ist jeweils oben in der Abbildung. Die richtige Lage zueinander ist entsprechend der vorher gezeigten Halbmodelle des A340-300 (siehe Abb. 42). 
Die Tabelle 13 enthält die in Abschnitt 2.3 beschriebenen Spezifikationen zu den Modellgrößen.

\begin{tabular}{|c|c|c|c|c|}
\hline $\begin{array}{c}\text { Anzahl } \\
\text { Strukturknoten }\end{array}$ & $\begin{array}{c}\text { Anzahl } \\
\text { Strömungsknoten }\end{array}$ & Stützstellen & $h_{X, \Omega}$ & $q_{X}$ \\
\hline 1823 & 66399 & Strukturknoten & 1.57668 & $6.71959 e-03$ \\
1823 & 66399 & Strömungsknoten & $5.19675 e-01$ & $1.14246 e-04$ \\
\hline
\end{tabular}

Tabelle 13. Die Modellgrößen der Anwendung.

Wie schon bei den vorherigen Anwendungsbeispielen sind die Berechnungsverfahren und Parameter zu den Abbildungen in der Tabelle $22 \mathrm{im}$ Anhang A aufgelistet.

Die an den Strömungs-Stützstellen angebrachten Kräfte stammen aus einer Strömungscode-Rechnung in einer Strömung-Struktur-Kopplung. Die Abbildungen 63 bis 65 zeigen die Ausgangskräfte und die über die Transponierte der mit der „Partition of Unity“ Methode erstellten Kopplungsmatrix transformierten Kräfte.

Die Ausgangskräfte und die mit Zusatzbedingungen zur Erhaltung der Arbeit und zur Konservativität interpolierten Kräfte werden in den Abbildungen 66 bis 68 präsentiert. Der Vergleich ergibt bei beiden Verfahren die Kraftkomponenten in $x$-,y- und $z$-Richtung von 5730.15, 14594.9 und -86775.4 und eine Arbeit für die drei Komponenten zusammen von 3266.219.

In den Abbildungen 63 bis 68 sind jeweils oben die Kräfte als Pfeile dargestellt und unten durch die Farbe, die den Betrag anzeigt. Die vertikale Legende bezieht sich auf die gegebenen Kräfte und die horizontale auf die interpolierten Kräfte in allen Abbildungen dieses Abschnitts. Als radiale Basisfunktion wurde die Volume Spline Funktion verwendet. Das Strukturgitter ist immer oben gezeigt.

Die Abbildung 69 zeigt die Ergebnisse der beiden Transformationsmethoden noch einmal untereinander mit einer anderen Pfeildarstellung.

Auch hier, wie beim AGARD Flügel, scheinen die Ergebnisse mit der Transponierten Matrix physikalisch sinnvoller zu sein. Es gibt zwar eine etwas größere negative Kraft beim Cockpit (auf Strukturseite/Bugfahrwerk), aber die Aerodynamik liefert auch entsprechende Werte. Bei der Methode mit den Zusatzbedingungen stört, dass in dem Rumpfteil hinter dem Flügel höhere Belastung auftritt (roter Bereich auf dem Rumpf des Strukturmodells). Der Verlauf der Kräfte sieht zwar sehr rund und verteilt aus, aber vielleicht sogar etwas zu sehr 'verteilt'. Im Bereich des Cockpits liefert nämlich die Aerodynamik ein paar Lastspitzen, die durch diese Methode wieder 'geglättet' werden. Bei der Methode mit der Transponierten werden die Spitzenwerte an Cockpit-Unterseite (wo das Bugfahrwerk ist) sowie am inneren Flügel, bzw. Flügelrumpf-Übergang besser und schärfer aufgelöst. 
114 Kapitel IV. Anwendungsbeispiele
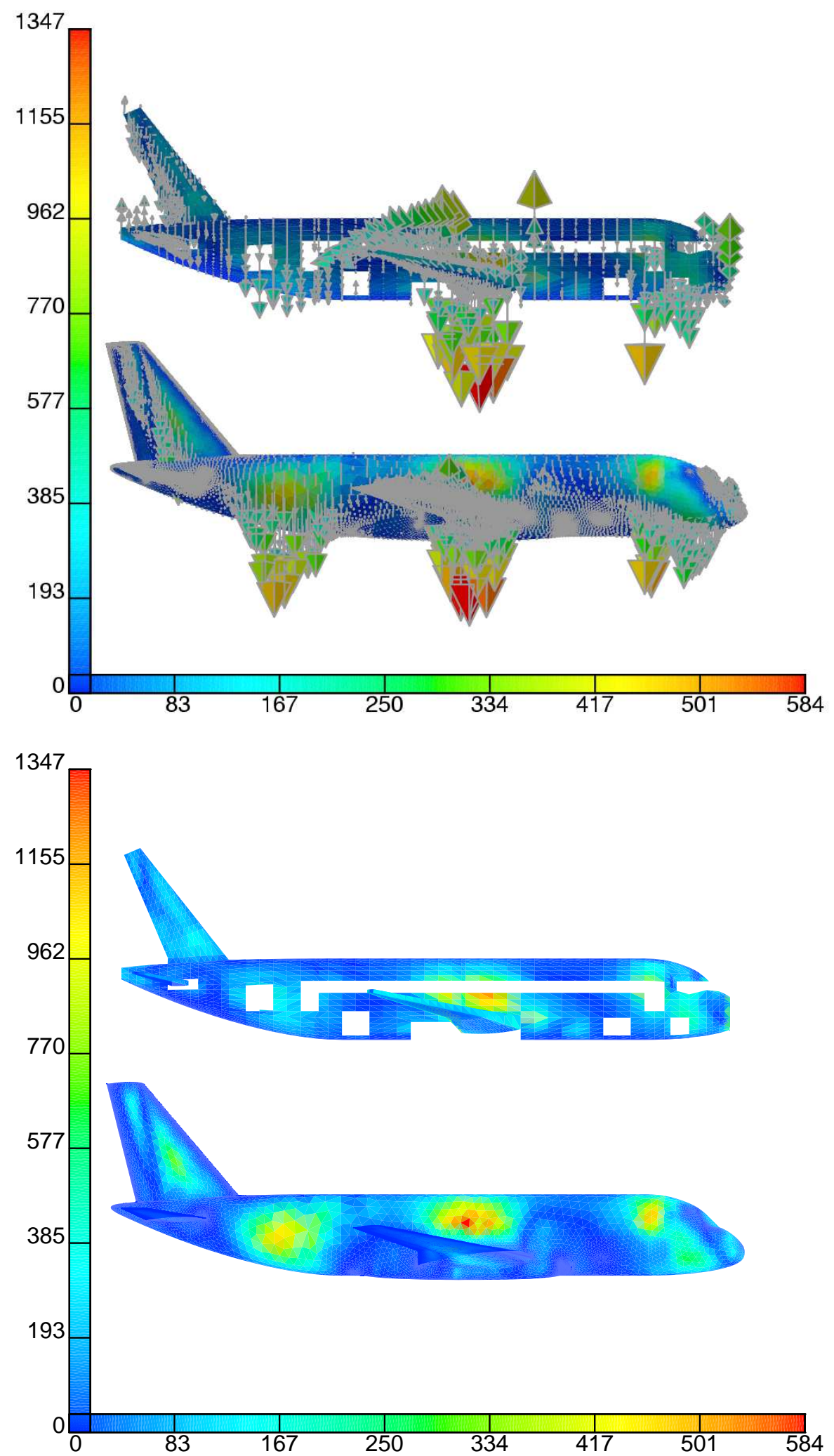

Abbildung 63. Die gegebenen und transformierten Kräfte. Die Ergebnisse der Transformation wurden mit der Transponierten der unter Verwendung von VS mit der PoU Methode berechneten Kopplungsmatrix erzielt. 

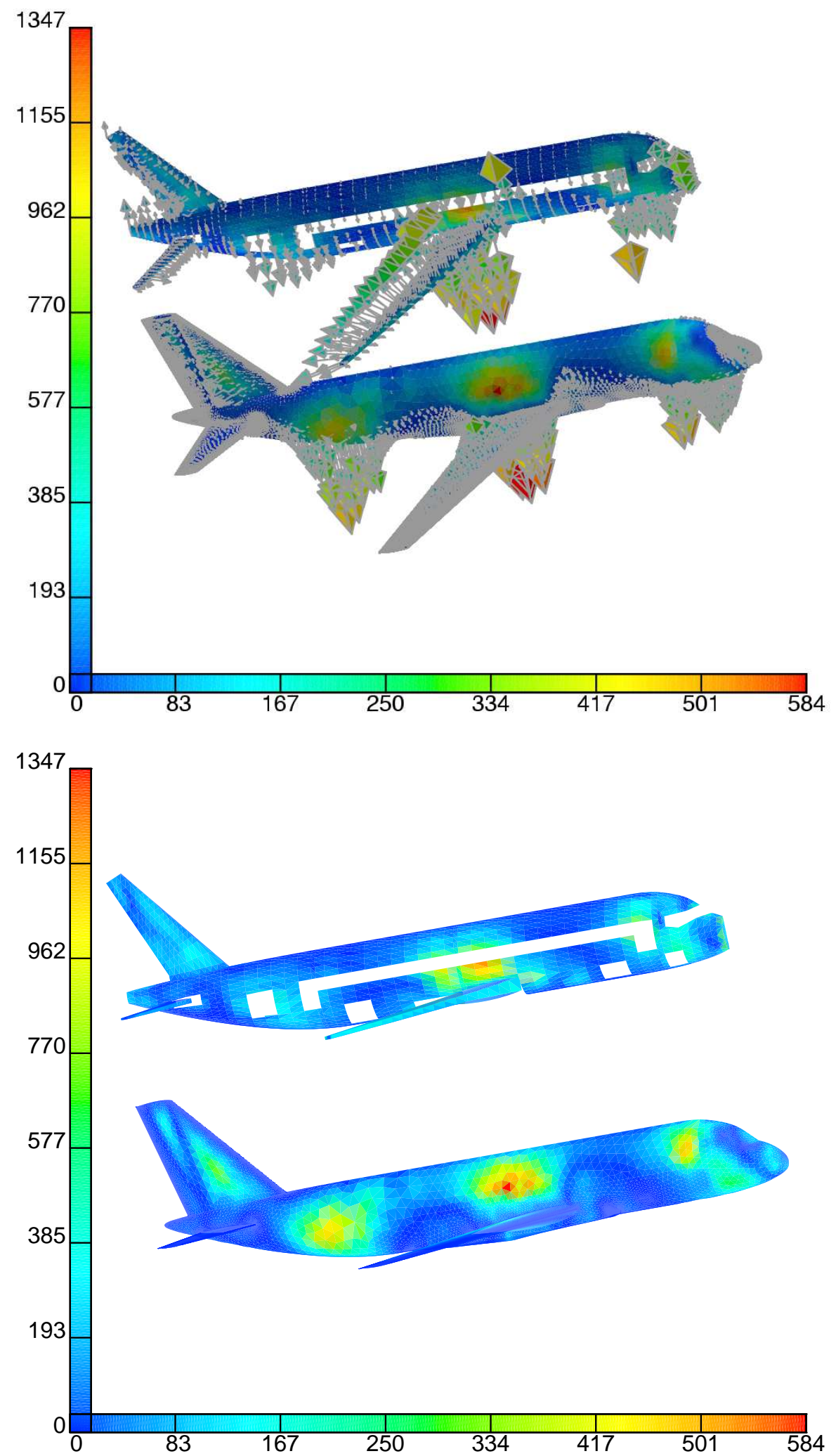

Abbildung 64. Die gegebenen und transformierten Kräfte. Die Ergebnisse der Transformation wurden mit der Transponierten der unter Verwendung von VS mit der PoU Methode berechneten Kopplungsmatrix erzielt. 

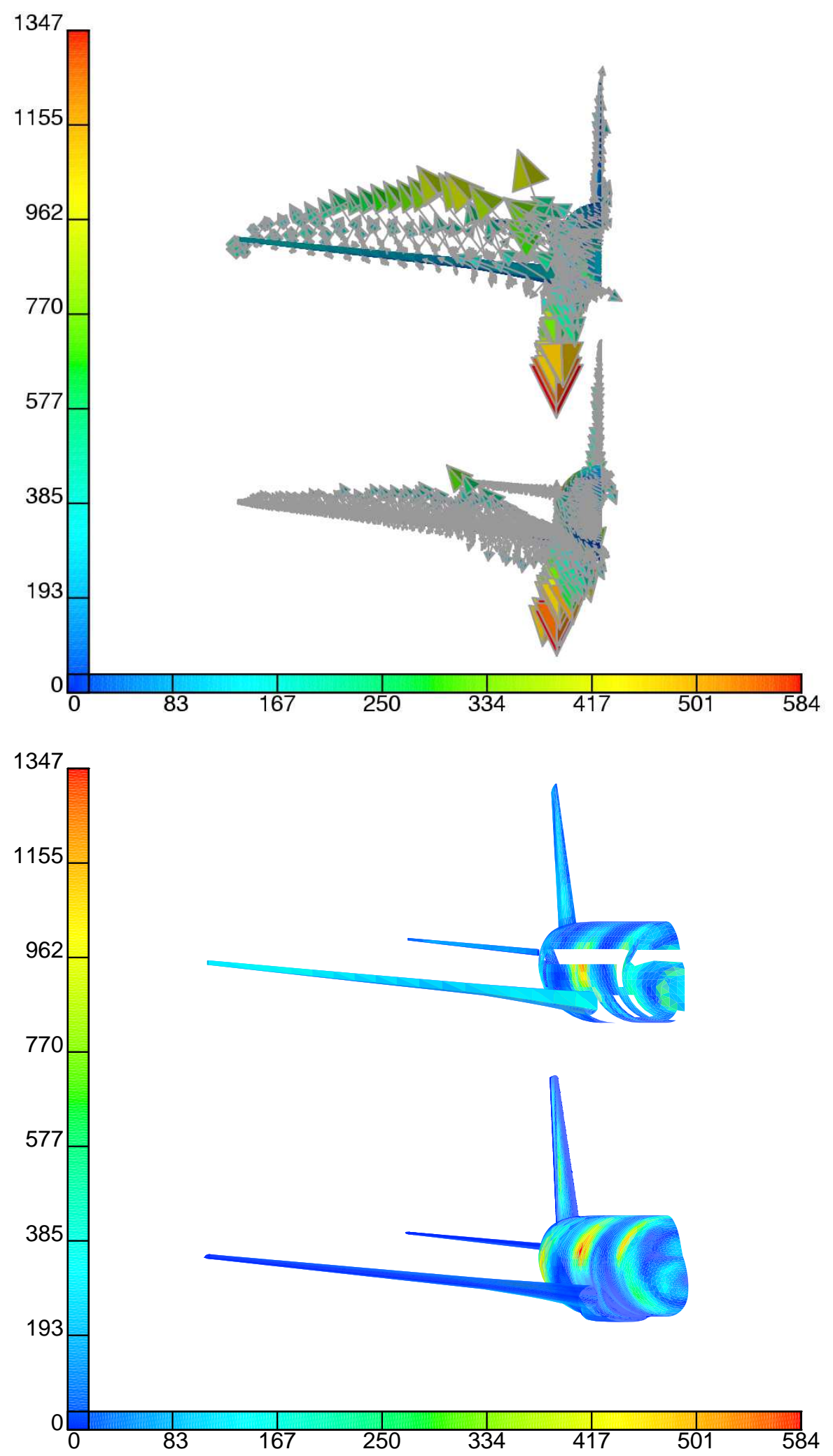

Abbildung 65. Die gegebenen und transformierten Kräfte. Die Ergebnisse der Transformation wurden mit der Transponierten der unter Verwendung von VS mit der PoU Methode berechneten Kopplungsmatrix erzielt. 

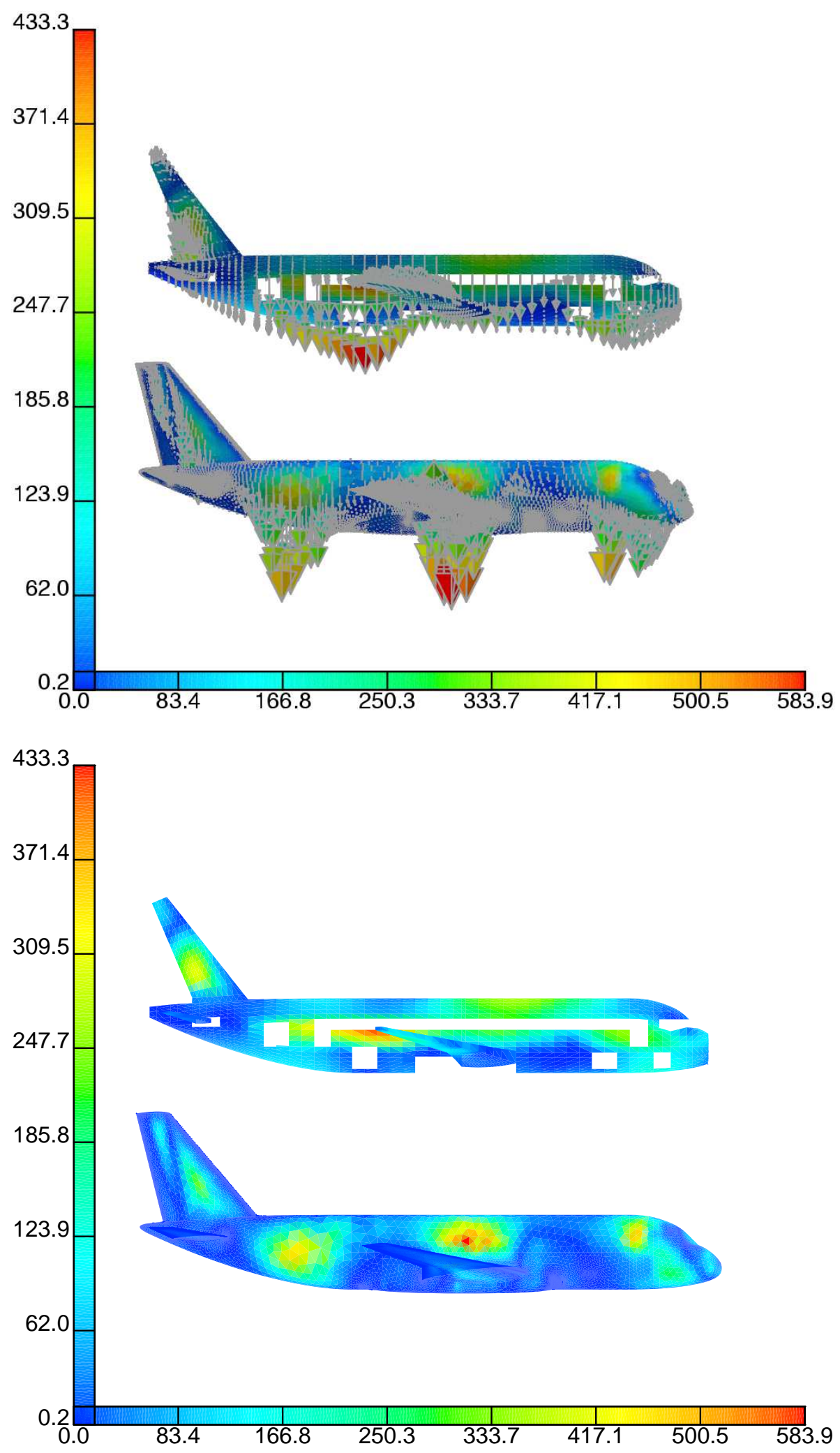

Abbildung 66. Die gegebenen und interpolierten Kräfte. Die Ergebnisse der Interpolation unter Verwendung von VS wurden mit der PoU Methode und Zusatzbedingungen zur Konservativität und zur Erhaltung der Arbeit erzielt. 

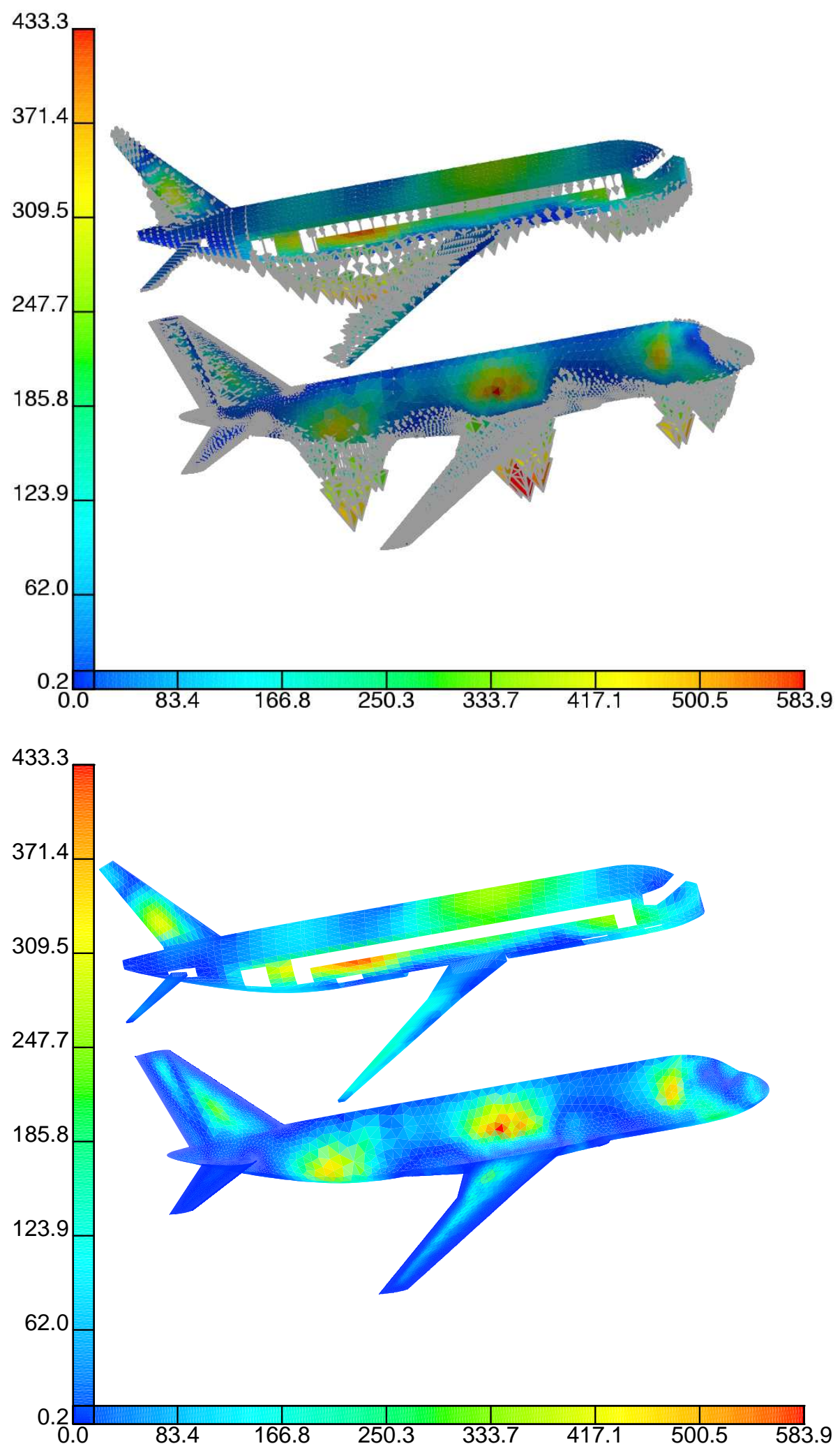

Abbildung 67. Die gegebenen und interpolierten Kräfte. Die Ergebnisse der Interpolation unter Verwendung von VS wurden mit der PoU Methode und Zusatzbedingungen zur Konservativität und zur Erhaltung der Arbeit erzielt. 

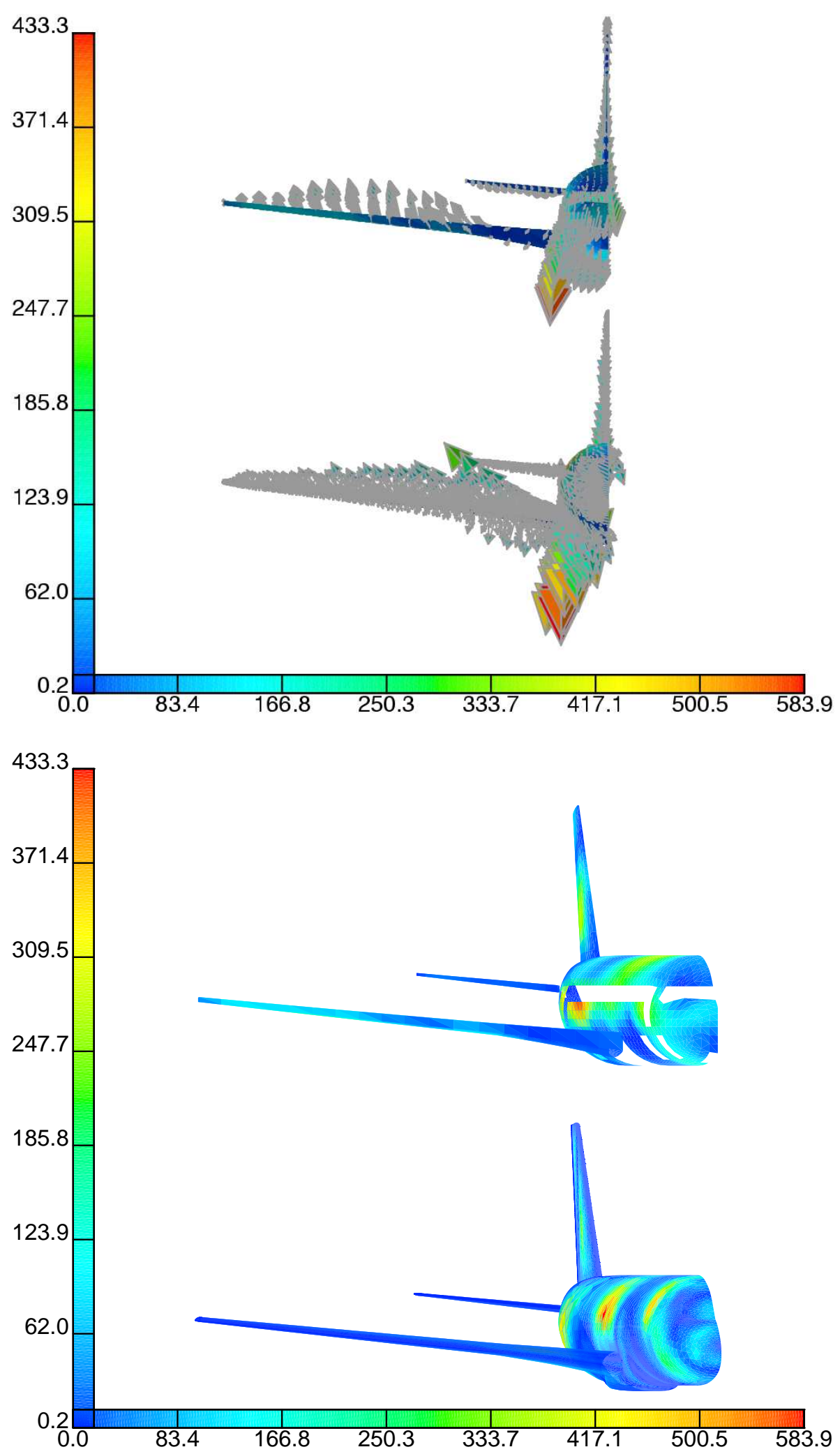

Abbildung 68. Die gegebenen und interpolierten Kräfte. Die Ergebnisse der Interpolation unter Verwendung von VS wurden mit der PoU Methode und Zusatzbedingungen zur Konservativität und zur Erhaltung der Arbeit erzielt. 

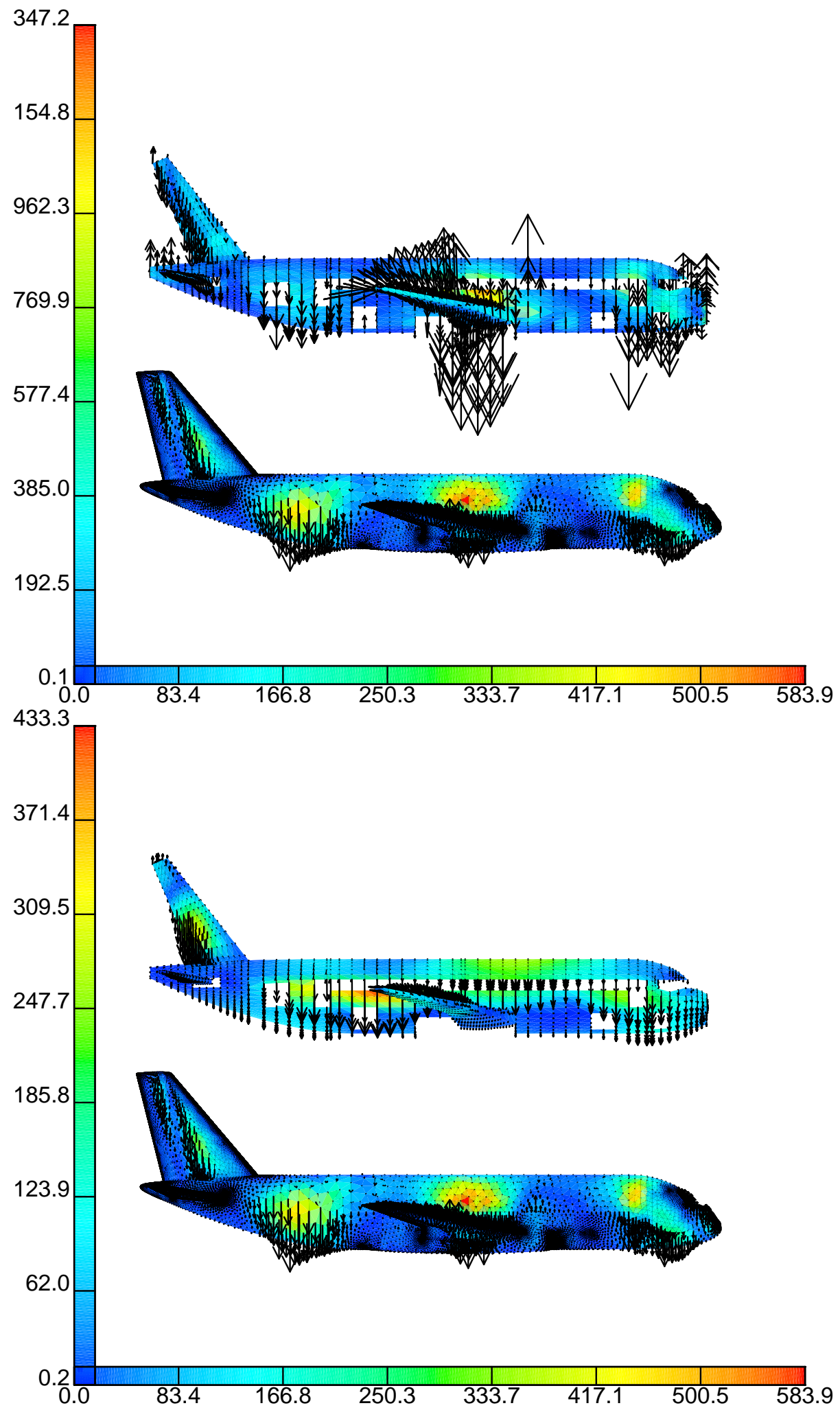

Abbildung 69. Die gegebenen und interpolierten Kräfte. Die Ergebnisse der Transformation auf der oberen Abbildung wurden mit der Transponierten der berechneten Kopplungsmatrix erhalten. Die Ergebnisse der Interpolation auf der unteren Abbildung wurden mit Zusatzbedingungen zur Konservativität und zur Erhaltung der Arbeit erzielt. Bei beiden Methoden wurde VS und die PoU Methode verwendet. 


\section{Anwendungen des ,Partition of Unity“" Verfahrens unter Einbeziehung der Rotationen}

\subsection{Strömung-Struktur-Kopplung am ALENIA Halbmodell}

Die Funktionalität der „Partition of Unity“ Methode unter Einbeziehung der Rotationen in die Interpolation wird in diesem Abschnitt an einem aeroelastischen Testbeispiel demonstriert. Das Anwendungsbeispiel ist wie im vorherigen Abschnitt das ALENIA Halbmodell, aber mit einer anderen Diskretisierung auf dem Strukturmodell. Das Struktur- und Strömungsgitter sind in Abbildung 70 dargestellt. Die Oberflächen bzw. Gitter sind zur besseren Darstellung in den Abbildungen mit einem vertikalen Abstand voneinander gezeigt. Die richtige Lage der Gitter zueinander ist in der Abbildung 70 rechts unten zu sehen. Die Struktur- und Strömungsmodelle sind aus verschiedenen Perspektiven dargestellt, wobei das Strukturmodell jeweils oben in den Abbildungen gezeigt ist.

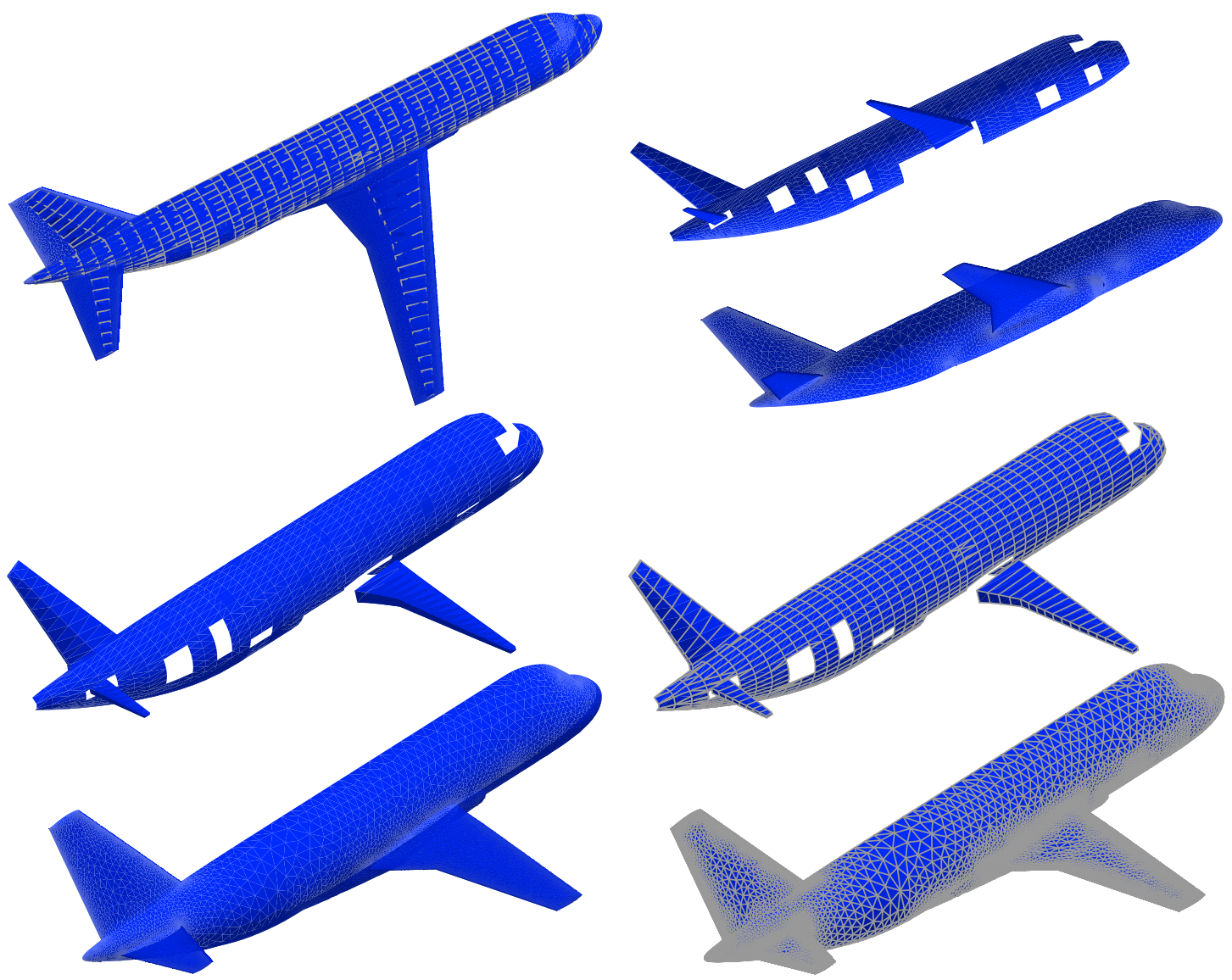

Abbildung 70. Das Struktur- und Strömungsgitter des Alenia-Halbmodells.

Die Tabelle 14 enthält die in Abschnitt 2.3 beschriebenen Spezifikationen zu den Modellgrößen. Die Berechnungsverfahren und Parameter der Abbildungen sind in der Tabelle 22 im Anhang A aufgelistet. 
Das Verhältnis von Strukturknoten zu Strömungsknoten ist nicht so extrem wie in dem Beispiel in Abschnitt 3.1, aber die Anzahl der Strukturknoten ist immer noch so gering im Vergleich zu den Strömungsknoten, dass sich das Beispiel für die Demonstration der Interpolation unter Einbeziehung der Rotationen eignet. Allerdings sind hier nicht nur Balkenelemente, die schnurförmig angeordnet sind, auf Strukturseite vorhanden, sondern dreiund viereckige Flächenelemente, die die Struktur erkennbar wiedergeben. So ist die Information über den Verlauf der Werte in alle Richtungen auch ohne Verwendung der Rotationen in den Interpolationen grundsätzlich gegeben. Dies ist ein qualitativer Unterschied zu dem Beispiel in Abschnitt 3.1 und erklärt die unten beschriebenen unterschiedlichen Ergebnisse beim Vergleich der Interpolationen mit und ohne Einbeziehung der Rotationen. Ein Beispiel, das groß genug war, um sinnvoll für die „Partition of Unity“ Methode zu sein und auf Strukturseite nur Balkenelemente enthielt, stand nicht zur Verfügung.

Die Deformationen bzw. Rotationen an den Stützstellen stammen aus einer Strukturcode-Rechnung in einer Strömung-Struktur-Kopplung. Die Ausgangsdeformationen auf den Strukturknoten und die mit Rotationen als Zusatzbedingungen interpolierten Deformationen auf den Strömungsknoten werden in den Abbildungen 71 und 72 präsentiert. Die Deformationen sind über die „Partition of Unity“ Methode unter Einbeziehung der Rotationen interpoliert worden. Die vertikale Legende bezieht sich auf die gegebenen Deformationen und die horizontale auf die interpolierten Deformationen. Als radiale Basisfunktion wurde die Wendland 2 Funktion verwendet. Das Strukturgitter ist wieder jeweils oben in den Abbildungen gezeigt. Zum Vergleich werden in den Abbildungen 73 und 74 die Ergebnisse der Interpolation der gleichen Deformationen ohne Einbeziehung der Rotationen bei Verwendung der gleichen radialen Basisfunktion und der gleichen Werte in allen anderen Parametern gezeigt. In der Abbildung 75 wird die Abweichung zwischen beiden Verfahren dargestellt. Die maximale Abweichung beträgt 0, 049767.

\begin{tabular}{|c|c|c|c|c|}
\hline $\begin{array}{c}\text { Anzahl } \\
\text { Strukturknoten }\end{array}$ & $\begin{array}{c}\text { Anzahl } \\
\text { Strömungsknoten }\end{array}$ & Stützstellen & $h_{X, \Omega}$ & $q_{X}$ \\
\hline 955 & 66399 & Strukturknoten & 1.77837 & 0.0145672 \\
\hline
\end{tabular}

Tabelle 14. Die Modellgrößen der Anwendung. 

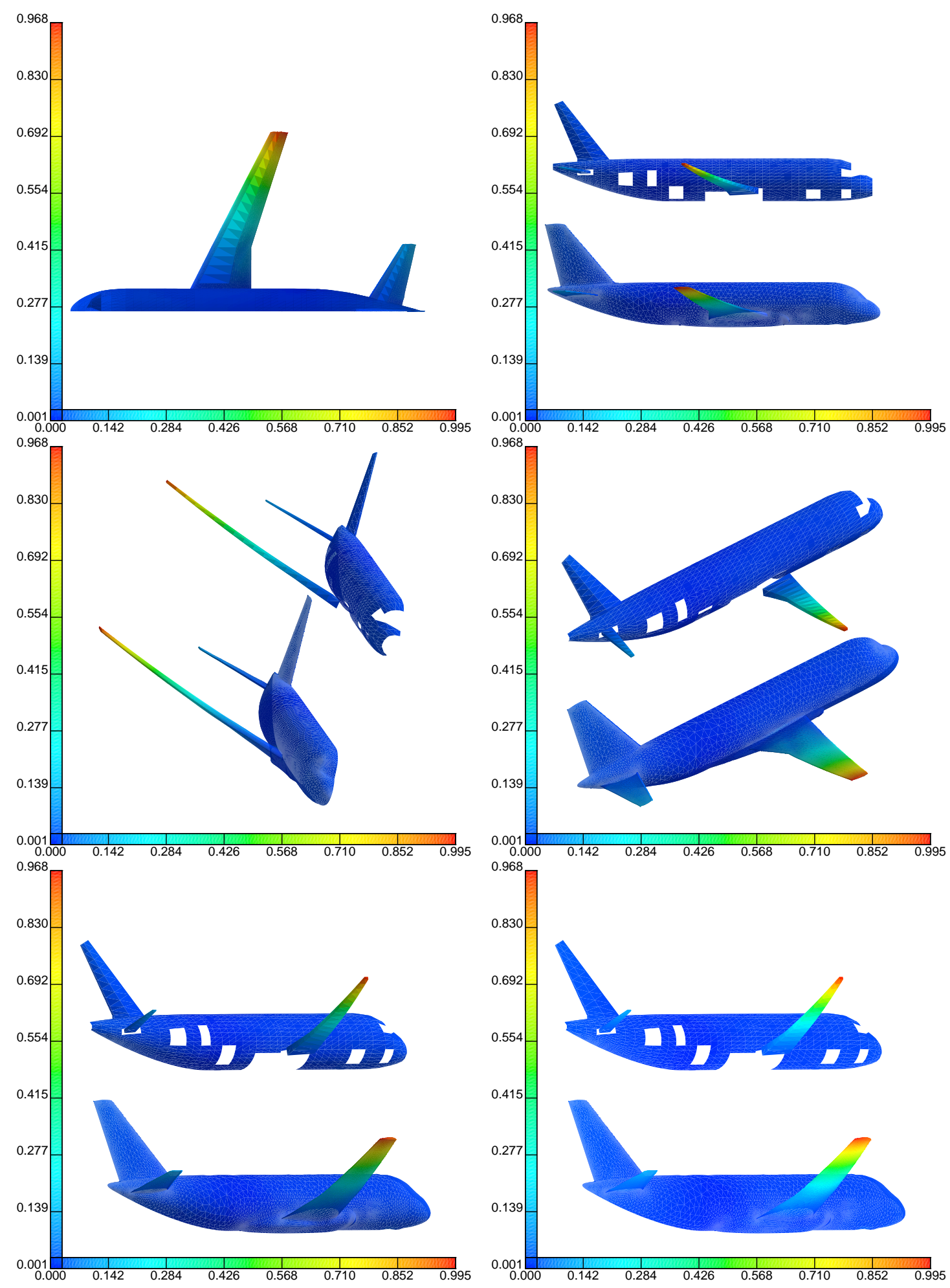

Abbildung 71. Die gegebenen und interpolierten Deformationen. Die Ergebnisse der Interpolation unter Verwendung von W2 wurden mit der PoU Methode und Rotationen als Zusatzbedingungen erzielt. 

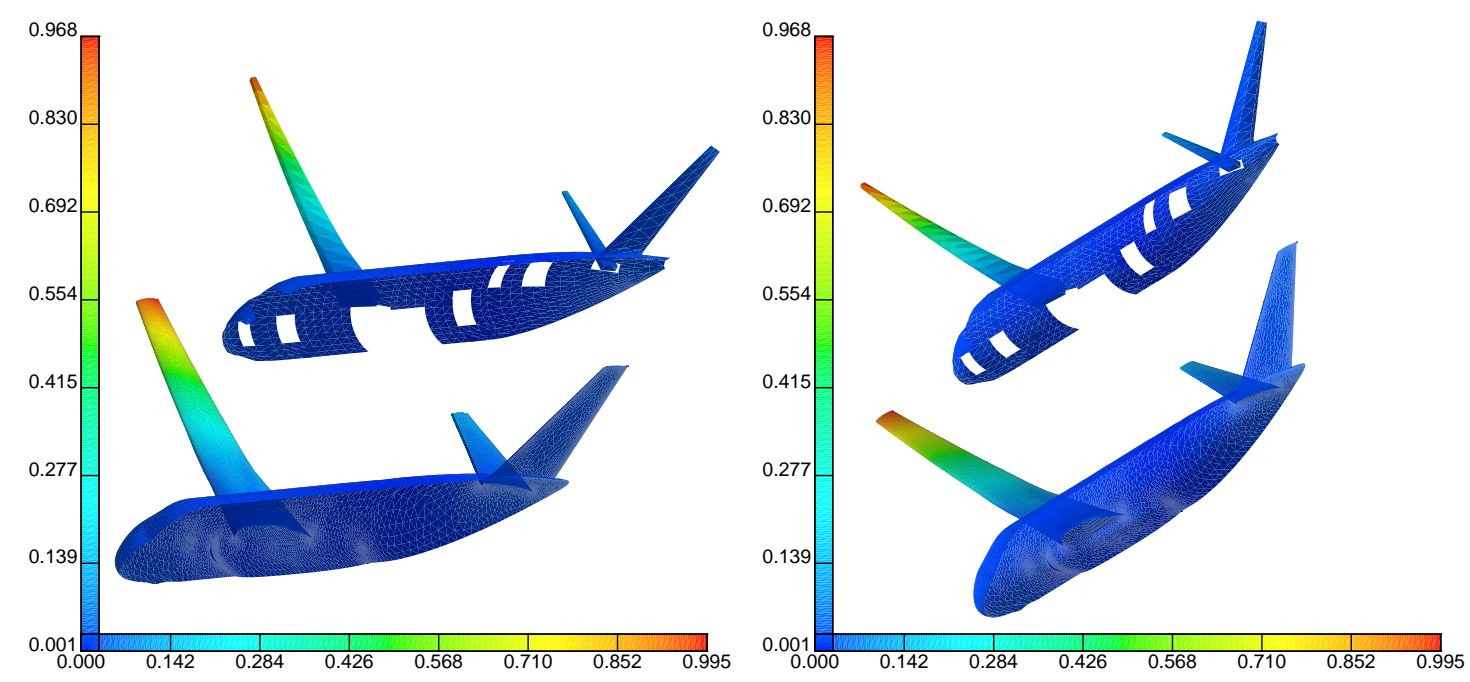

Abbildung 72. Die gegebenen und interpolierten Deformationen. Die Ergebnisse der Interpolation unter Verwendung von W2 wurden mit der PoU Methode und Rotationen als Zusatzbedingungen erzielt.
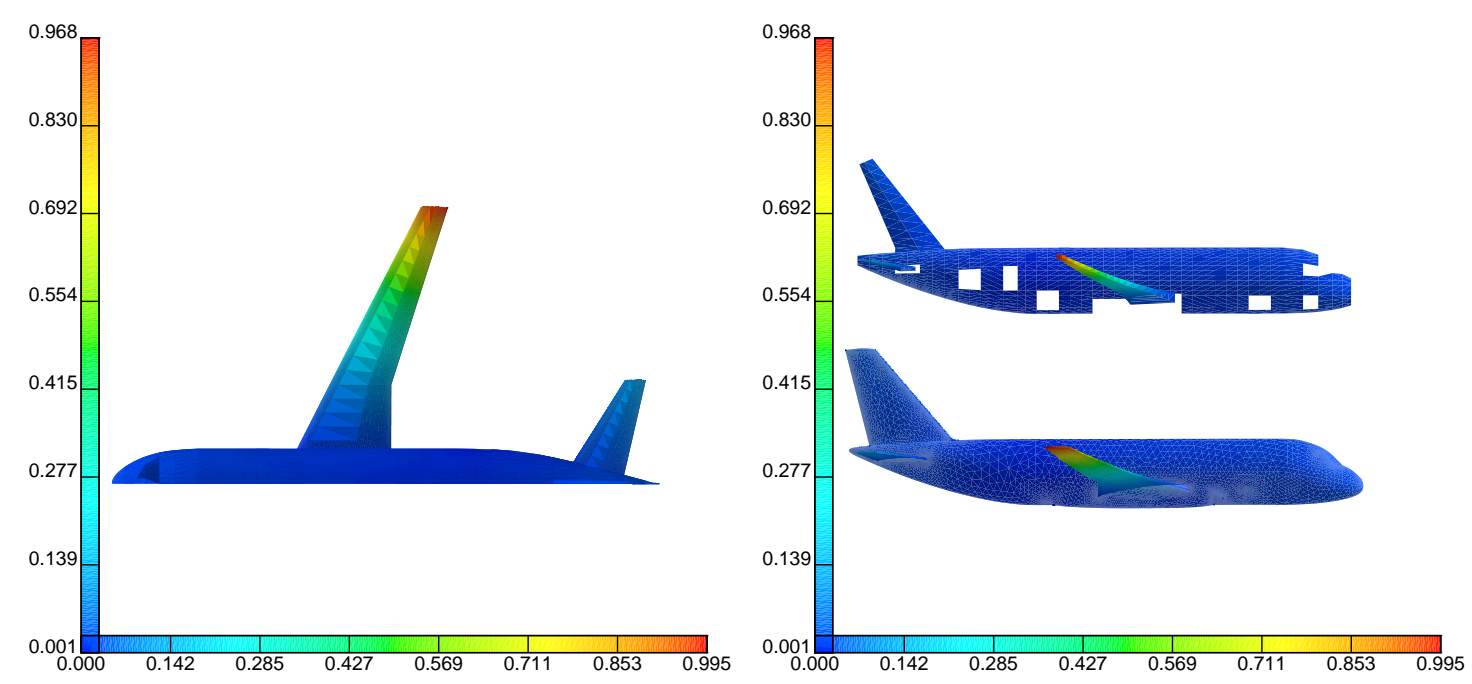

Abbildung 73. Die gegebenen und interpolierten Deformationen. Die Ergebnisse der Interpolation unter Verwendung von W2 wurden mit der PoU Methode ohne Rotationen als Zusatzbedingungen erzielt. 

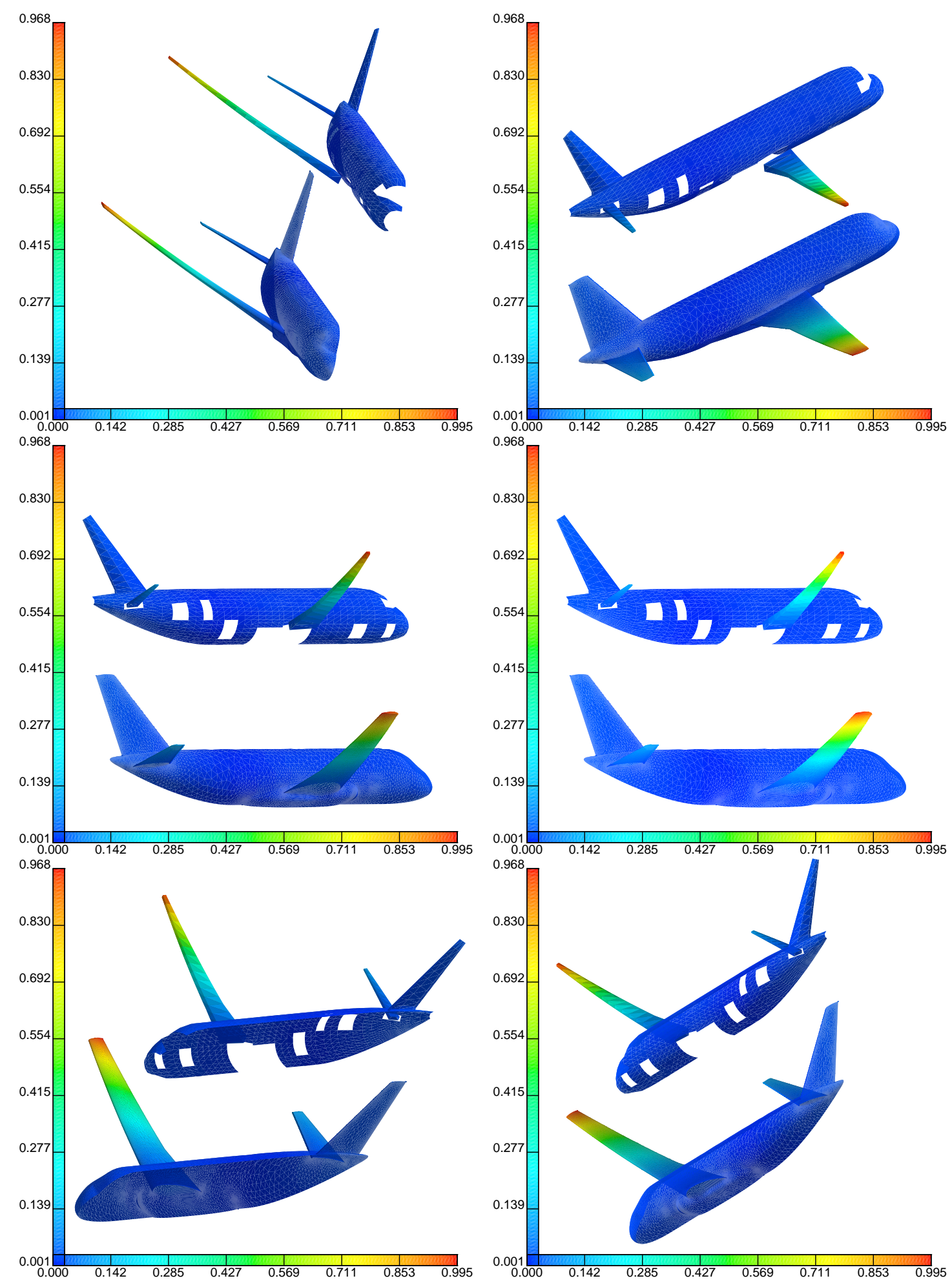

Abbildung 74. Die gegebenen und interpolierten Deformationen. Die Ergebnisse der Interpolation unter Verwendung von W2 wurden mit der PoU Methode ohne Rotationen als Zusatzbedingungen erzielt. 

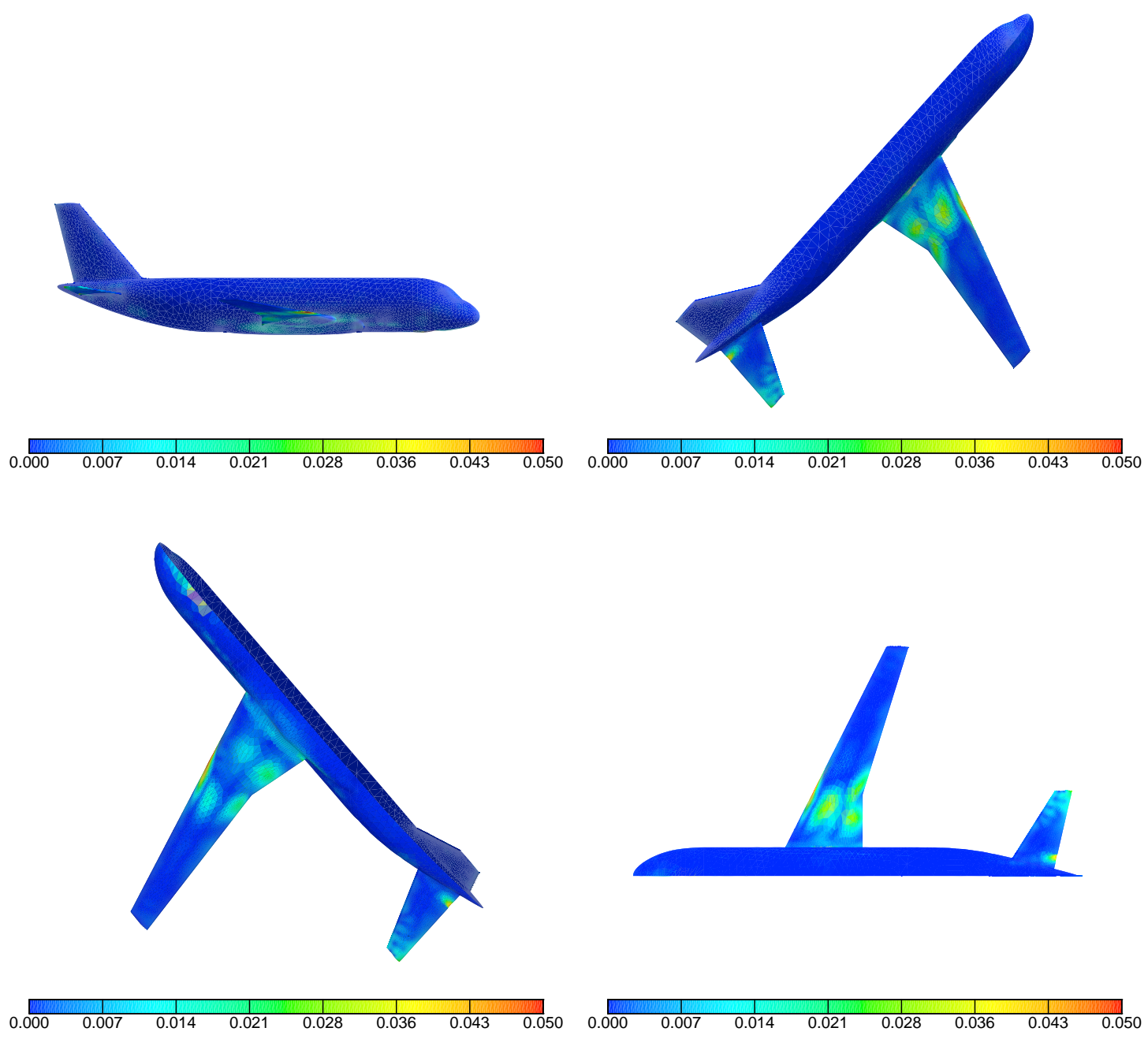

Abbildung 75. Die Abweichungen zwischen den Ergebnissen der interpolierten Deformationen mit und ohne Rotationen als Zusatzbedingungen. Die interpolierten Deformationen wurden in beiden Verfahren unter Verwendung von W2 mit der PoU Methode erzielt.

Die Anwendung zeigt wieder, dass die Interpolation unter Verwendung der Rotationen als Zusatzbedingungen physikalisch relevante Ergebnisse liefert. An den Differenzen ist zu sehen, dass hier die Abweichungen durch numerische Probleme in der selben Größenordnung liegen, wie die durch die Methode selbst hervorgerufenen systematischen Abweichungen. Die Abweichungen durch die Methode sind bei diesem Beispiel aus den oben genannten Gründen nicht so deutlich, wie im Beispiel in Abschnitt 3.1, auf der Abbildung 75 sind aber andeutungsweise die selben farblichen Strukturen bei den Abweichungen zu sehen wie auf der Abbildung 39 und 40 in Abschnitt 3.1. 


\section{Gekoppelte Simulation in der Aeroelastik am ALENIA Halbmodell}

\subsection{Das Strömung-Struktur-Kopplungsverfahren}

In den vorherigen Abschnitten dieses Kapitels wurde die Anwendung aller entwickelten und implementierten Interpolationsalgorithmen einzeln an verschiedenen Beispielen demonstriert. In diesem Abschnitt wird zum Abschluss eine gekoppelte Simulation unter Verwendung von in dieser Arbeit entwickelten Interpolationsalgorithmen präsentiert. Diese Simulation und ihre Auswertung sind bei EADS Military Aircraft im Rahmen des europäischen Projekts TAURUS (Technology Development for Aeroelastic Simulations on Unstructured Grids) durchgeführt worden und waren auch Thema einer Diplomarbeit [25]. Das Projekt TAURUS beschäftigt sich mit der Entwicklung einer effizienten, benutzerfreundlichen Simulationstechnologie zur Strömung-StrukturKopplung in der Aeroelastik.

Da die Durchführung einer kompletten gekoppelten Simulation sehr aufwendig ist und daher nicht alle neuen Interpolationsverfahren in einer gekoppelten Simulation getestet werden können, sind zunächst die Algorithmen für die Kopplung ausgewählt worden.

Da sich in den vorherigen Beispielen gezeigt hat, dass die Verwendung der transponierten Kopplungsmatrix zur Transformation der Kräfte zu physikalisch sinnvolleren Ergebnissen führt, als die Verwendung der Interpolation auf Kräfteseite mit Zusatzbedingungen, kommt als Verfahren für das hier vorliegende Problem damit die Interpolation der Deformationen mit der „Partition of Unity" Methode und die Transformation der Kräfte mit der transponierten Matrix zum Einsatz. Da das simulierte Problem eine Größe hat, die hinsichtlich der Deformationen noch global mit radialen Basisfunktionen interpoliert werden kann, können Vergleiche zwischen globaler Interpolation und „Partition of Unity" Methode gezogen werden. Die Transformation der Kräfte wird dabei auch bei der globalen Interpolation über die Transponierte der Kopplungsmatrix für die Deformationen realisiert.

Das ausgewählte Verfahren ist auch hinsichtlich der Rechenzeit der anderen Methode vorzuziehen. Da ein statisches aeroelastisches Problem simuliert wird, für welches die Gitter nicht verändert werden, müssen die Transformationsmatrizen nicht in jedem Kopplungsschritt neu aufgebaut werden. So wird das Verfahren, welches auf Grund des Algorithmus die Transformationsmatrizen nur einmal aufzustellen braucht und diese Transformationsmatrizen für jeden Kopplungsschritt nutzen kann, im Bezug auf die Rechenzeit immer im Vorteil sein.

Bei einem dynamischen Problem mit verschobenen Gittern oder adaptierten Gitterstrukturen, wäre dieser Vorteil nicht mehr gegeben. Bei veränderlichen Gittern müssen die Matrizen bei jedem Interpolationsschritt nach Veränderung der Gitterknoten neu aufgestellt werden; unter diesen Bedingungen würde sich kein Nachteil hinsichtlich der Rechenzeit ergeben, wenn 
Verfahren verwendet werden, die die Kopplungsmatrizen in jedem Schritt neu berechnen.

Die Rotationen als Zusatzbedingungen statt des ausgewählten Verfahrens zu testen, ist bei der vorliegenden Konfiguration in der gekoppelten Simulation nicht empfehlenswert. Die Anzahl und Lage der Strukturknoten wäre nicht ideal, um Unterschiede zwischen Interpolationen mit und ohne Rotationen als Zusatzbedingungen herauszuarbeiten.

Zunächst folgen einige Erläuterungen zu dem in der Kopplung simulierten Problem. Wie schon in der Einleitung beschrieben, beschäftigen sich die Probleme in der Aeroelastik mit der Wechselwirkung zwischen der durch die Strömungskräfte verformten, elastischen Struktur und der durch die Strukturveränderung beeinflussten Strömung. Ziel der Simulation ist die Vorhersage des statischen aeroelastischen Deformationsverhaltens dreidimensionaler Tragflügelstrukturen. Die Strömung-Struktur-Kopplung wird bei dem hier verfolgten Ansatz durch die getrennte Berechnung der beiden unterschiedlichen physikalischen Probleme mit einer losen Kopplung realisiert. Die beiden monodisziplinären Feldprobleme werden bekanntlich durch partielle Differentialgleichungen beschrieben.

Strukturgleichungen Die Struktur wird repräsentiert durch die Lagrange Bewegungsgleichungen, die für eine endliche Anzahl von Lagrange Koordinaten und ein lineares elastische Material durch

$$
\sum_{p=1}^{N} \hat{M}_{n p} \ddot{\hat{q}}_{p}(t)+\sum_{p=1}^{N} \hat{K}_{n p} \hat{q}(t)=\hat{e}_{n} \quad 1 \leq n \leq N
$$

beschrieben wird, unter der Vorraussetzung, dass die Verschiebungen durch

$$
u\left(\xi^{\alpha}, t\right)=\sum_{n=1}^{\infty} \hat{q}_{n}(t) \hat{\psi}_{n}\left(\xi^{\alpha}\right)
$$

auszudrücken sind, wobei $\hat{\psi}_{n}\left(\xi^{\alpha}\right)$ die natürlichen Moden der Strukturschwingung und $\hat{q}_{n}(t)$ die Lagrange Variablen sind, $\hat{M}_{n p}$ die Massenmatrix, und $\hat{K}_{n p}$ ist. $\hat{e}_{n}$ sind die Kräfte auf die Massenpunkte.

Die allgemeinen Strukturgleichungen werden mit einer EADS-M Inhouse Software auf Basis der Finite Elemente Methode gelöst. Das Strukturproblem lässt sich dann durch die klassische lineare Formulierung des elastostatischen Gleichgewichtszustand

$$
K u=f
$$

beschreiben, wobei $u$ der Verschiebungsvektor der Strukturknoten, $K$ die globale Steifigkeitsmatrix und $f$ der Lastvektor mit den durch die Strömung erzeugten Kräften ist, die auf die Strukturknoten einwirken. 
Strömungsgleichungen Die Gleichungen der reibungsfreien Strömungsmechanik leiten sich aus den physikalischen Grundprinzipien der Massen-, Impuls- und Energieerhaltung ab. Die daraus herzuleitenden partiellen Differentialgleichungen sind bei Vernachlässigung der Reibungsterme vom hyperbolischen Typ. Eine transsonische, reibungsfreie Strömung wird durch die dreidimensionalen Eulergleichungen für ein raumfestes Volumen $V=V_{0}$

$$
\int_{V_{0}}\left[\frac{\partial U}{\partial t}+\frac{\partial F}{\partial x}+\frac{\partial G}{\partial y}+\frac{\partial H}{\partial z}\right] d V=0
$$

beschrieben, wobei

$$
\begin{aligned}
U= & {\left[\begin{array}{c}
\rho \\
\rho u \\
\rho v \\
\rho w \\
\rho e_{g e s}
\end{array}\right], F=\left[\begin{array}{c}
\rho u \\
\rho u^{2}+p \\
\rho u v \\
\rho u w \\
\rho u\left(e_{\text {ges }}+\frac{p}{\rho}\right)
\end{array}\right], G=\left[\begin{array}{c}
\rho v \\
\rho v u \\
\rho v^{2}+p \\
\rho v w \\
\rho v\left(e_{g e s}+\frac{p}{\rho}\right)
\end{array}\right], } \\
H= & {\left[\begin{array}{c}
\rho w \\
\rho w u \\
\rho w v \\
\rho w^{2}+p \\
\rho w\left(e_{g e s}+\frac{p}{\rho}\right)
\end{array}\right] . }
\end{aligned}
$$

In den Gleichungen sind $\rho$ die Dichte, $u, v$ und $w$ die Komponenten des Geschwindigkeitsvektors und $p$ der Druck. Die auf die Masse bezogene Gesamtenergie $e_{\text {ges }}$ ergibt sich aus der inneren Energie und der kinetischen Energie.

Unstetigkeiten in der Strömung, bei der sich Zustandsgrößen oft um mehrere Größenordnungen ändern und die typisch für transsonische und Übersowie Hyperschallströmungen sind, sind in diesen Gleichungen mit erfasst. Die Lösung der Euler-Gleichungen gelingt im Allgemeinen nur numerisch, durch Diskretisierung des betrachteten dreidimensionalen Strömungsfeldes und der Zeit.

In der hier betrachteten gekoppelten Simulation wird zur Berechnung des Strömungsproblems der TAU-Code (siehe [26]) verwendet, ein vom DLR Institut für Aerodynamik und Strömungsmechanik entwickelter und vertriebener CFD Code.

Im TAU-Code werden die Euler-Gleichungen mit einer Finite Volumen Methode gelöst. Der entscheidende Vorteil dieser Methode ist, dass unstrukturierte Gitter verwendet werden können. Durch die mit der Finite Volumen Methode erhaltenen Gleichungen werden die drei grundlegenden Erhaltungssätze, die Erhaltung der Masse, des Impulses und der Energie, vom kontinuierlichen Raum auf den diskreten Raum übertragen.

Struktur- und Strömungsmodell Die gekoppelte Simulation wird an dem Modell eines zivilen Transportflugzeugs durchgeführt, welches wieder von 
Alenia Aeronautica zur Verfügung gestellt wurde. Das Modell besteht nur aus der rechten Hälfte des Flugzeugs, da in den Testfällen nur symmetrische Manöver und der statische horizontale Flugzustand behandelt werden.

Das Strukturmodell besteht aus 2816 Strukturknoten und 6194 ein- und zweidimensionalen linearen Elementen, wie in Abbildung 76 dargestellt.

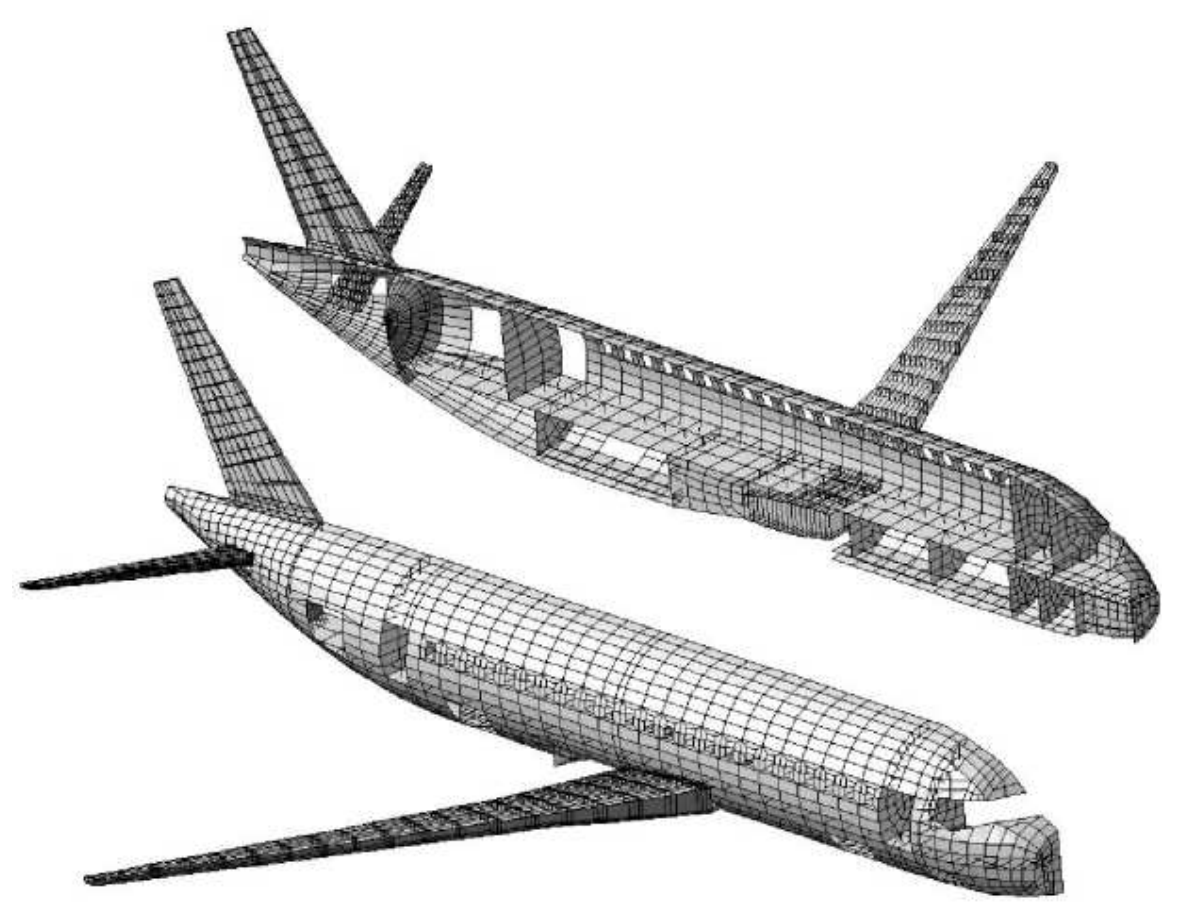

Abbildung 76. Das Finite Elemente Modell des Alenia Testflugzeugs.

Das Strömungsfeld wird durch 1,9 Millionen Tetraeder Elemente diskretisiert. Das äußere Feld und das Oberflächengitter des vom DLR zur Verfügung gestellten Strömungsgitters werden in Abbildung 77 und 78 gezeigt. Die Unterschiede zwischen Strömungs- und Strukturmodell an der Oberfläche des Flugzeugs zeigen sich bereits, wenn beide Oberflächengitter wie in Abbildung 79 übereinander gelegt werden. Die Ursachen für die Verwendung unterschiedlicher Gitter sind bereits ausführlich in Kapitel I und III erörtert worden. Bei der hier präsentierten Strömung-Struktur-Kopplung ergeben sich die verschiedenen Gitter aus den unterschiedlichen Diskretisierungen der Finite Volumen Methode und des Finite Elemente Verfahrens zum einen und zum anderen daraus, dass geometrische Details wie Fenster und Türen für 


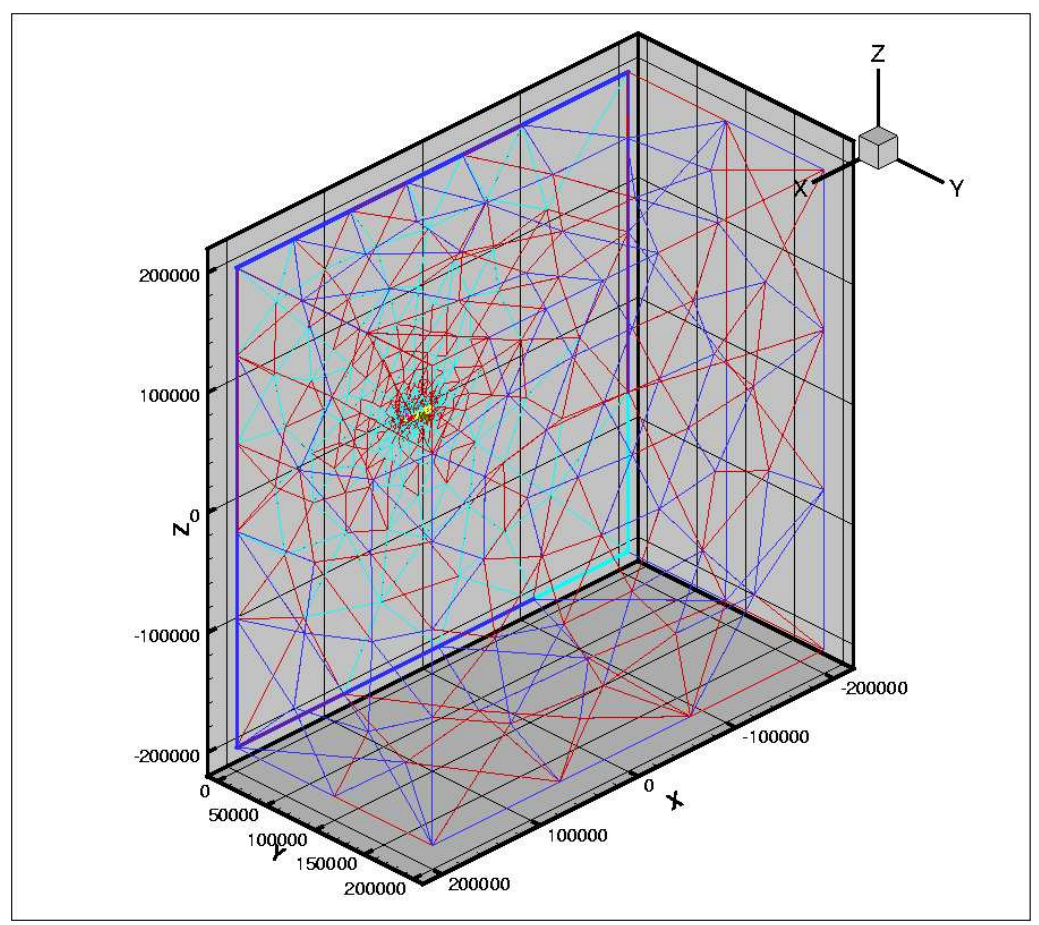

Abbildung 77. Das äußere Feld des Strömungsgitters.

die Struktur im Gegensatz zur Strömung von Bedeutung für die Simulation sind.

Die Interpolation bei der Strömung-Struktur-Kopplung in der Aeroelastik muss verschiedene Charakteristika haben, um einen sinnvollen Austausch von Deformationen und Kräften zu gewährleisten. Diese Anforderungen sind in der folgenden Liste noch einmal zusammenfassend aufgeführt und kurz erläutert:

- Die Struktur- und Strömungsknoten müssen beliebig verteilt sein können, da durch die unterschiedlichen Diskretisierungen beliebige Verteilungen der Knoten entstehen können.

- Es darf keine strukturellen Anforderungen an das Struktur- und Strömungsgitter geben, da dies die Möglichkeiten der Simulationscodes einschränken würde.

- Das Verhältnis der Anzahl der Strukturknoten zur Anzahl der Strömungsknoten muss beliebig sein, da auch hier unterschiedliche Kombinationen von Gittern auftreten können. In den meisten Anwendungen ist das Strukturgitter wesentlich gröber als das fein aufgelöste Strömungsgitter.

- Die Interpolationen müssen auch bei großen, sehr fein aufgelösten Gittern anwendbar sein ohne zu immensen Rechenzeiten zu führen.

- Lokale Effekte wie Stöße und Strukturkrümmungen sollten nicht über eine größere Fläche verschmiert werden, sondern auch nach der Interpolation im anderen Feld einen lokalen Effekt darstellen.

- Die Interpolationen müssen folgende Zusatzbedingungen erfüllen: 


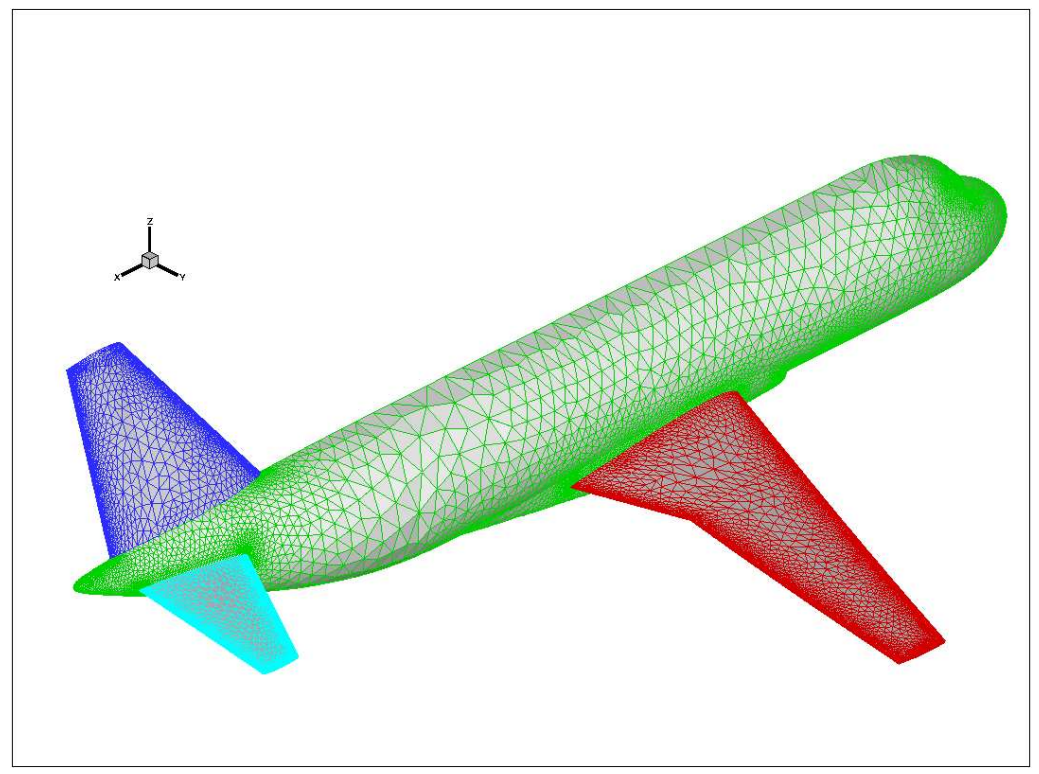

Abbildung 78. Das Oberflächengitter des Strömungsgitters.

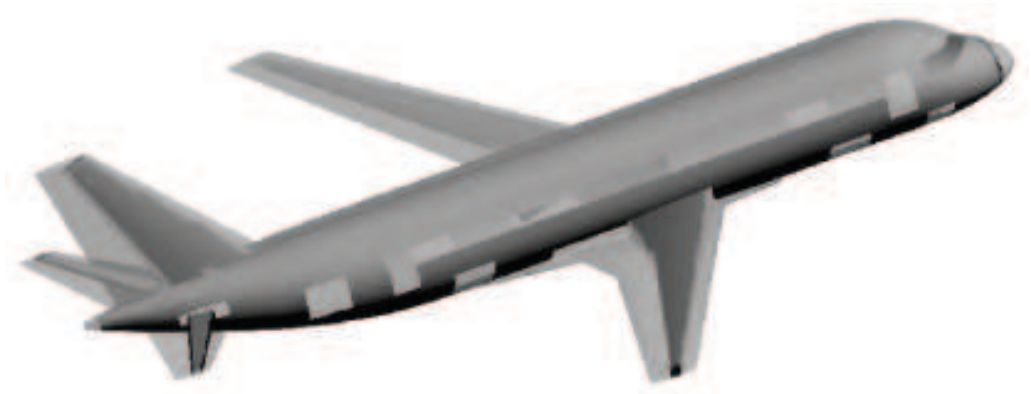

Abbildung 79. Das Strömungs- und Strukturmodell des Alenia Flugzeugs.

- Erhaltung der Arbeit, die durch die Summe der Kräfte multipliziert mit den Deformationen über alle Oberflächenknoten des Flugzeugs gebildet wird und in statischen und dynamischen Anwendungen auf Strömungs- und Strukturseite gleich sein muss (siehe Kapitel II Abschnitt 2.1), um eine arbeitsäquivalente Deformation zu erzielen.

- Konservativität, d.h. dass die Summe der Kräfte und Momente über alle Oberflächenknoten des Flugzeugs auf Strömungs- und Strukturseite gleich ist (siehe Kapitel II Abschnitt 2.2). Diese Bedingung ist erforderlich, um eine physikalisch sinnvolle Verteilung der Lasten zu erhalten.

- Die Interpolationsfunktion muss so glatt sein, dass eine Nachglättung, die die Zusatzbedingungen der Interpolation nicht mehr gewährleisten und zusätzlichen Rechenaufwand benötigen würde, nicht notwendig ist.

- Die Algorithmen müssen stabil und robust funktionieren. 
Diese Bedingungen begründen die Anwendung der radialen Basisfunktionen bei der Strömung-Struktur-Kopplung in besonderem Maße, da die radialen Basisfunktionen wie keine andere Interpolation diese Bedingungen erfüllen.

Lösungsalgorithmus Bei der präsentierten Simulation werden bezüglich der Struktur keine Nichtlinearitäten beachtet, in der Strömung müssen aber nichtlineare Effekte berücksichtigt werden, welches einen iterativen Lösungsprozess erfordert. Das hier verwendete Verfahren ist der Conventional Serial Staggered (CSS) Algorithmus (siehe [3]). Bei diesem Verfahren werden im ersten Iterationsschritt zunächst die aktuellen Verformungen von der Struktur an die Strömung gegeben, dann wird der Strömungscode mit dieser deformierten Geometrie die neuen Drücke, die auf die Struktur wirken, berechnen. Durch Integration der Drücke über die entsprechenden Flächen werden die Kräfte für die Flächenmittelpunkte ermittelt. Im dritten Schritt werden die Kräfte vom Strömungscode an den Strukturcode gegeben und auf die Knoten des undeformierten Strukturgitters gegeben. Der Strukturcode berechnet im vierten Iterationsschritt aus diesen Kräften eine neue Deformation. Dann startet der Algorithmus wieder mit dem ersten Iterationsschritt. Die Iterationsschritte sind in Abbildung 80 skizziert. Dieses Verfahren wird so lange

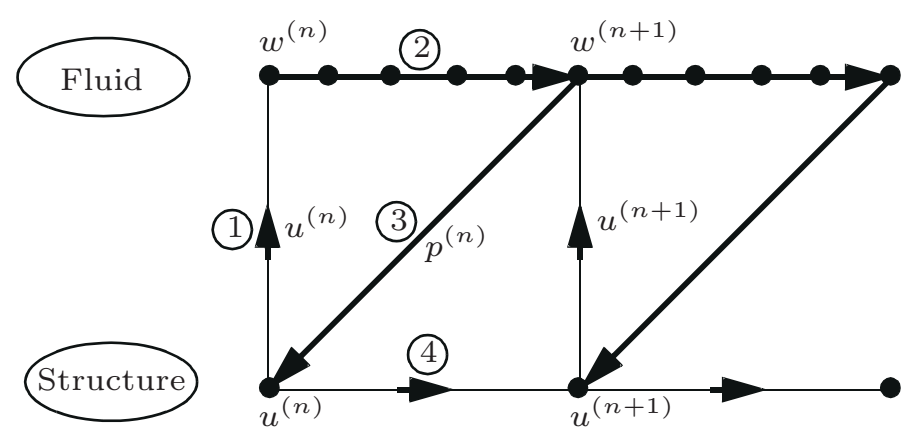

Abbildung 80. Skizze des Conventional Serial Staggered Algorithmus.

wiederholt, bis die Änderung der Verformung von einem Durchlauf des Verfahrens zum nächsten unter einer festgesetzten Schranke $\epsilon$ liegen. Wird also

$$
e r r=\sqrt{n_{x}^{2}+n_{y}^{2}+n_{z}^{2}}
$$

gesetzt, wobei $n_{x}$, und entsprechend $n_{y}, n_{z}$, gegeben ist als

$$
n_{x}=\left|\frac{u_{x_{\max }}^{(i)}-u_{x_{\max }}^{(i-1)}}{u_{x_{\max }^{(i-1)}}^{(i)}}\right|,
$$

wobei $u_{x_{\max }}^{(i)}$ der maximale Wert der Deformation in $x$-Richtung bei dem $i$-ten Durchlauf ist, so wird abgebrochen, falls err $<\epsilon$ gilt. 


\subsection{Simulationsgrundlagen}

Die Simulationen sind mit folgenden Flug- und Strömungsbedingungen durchgeführt worden:

Mach 0.8

Temperatur $216.65 \mathrm{~K}$

Druck 21662.68 Pa

dynamischer Druck 9704.9 Pa

Wie oben beschrieben wird die Lösung mit einem iterativen Verfahren erzielt, bei welchem im ersten und dritten Iterationsschritt Daten ausgetauscht werden. Wie bereits erwähnt werden die Deformationen mit Hilfe der Interpolation transportiert, während für die Kräfte die Transponierte der Kopplungsmatrix benutzt wird. Die im Strömungscode berechneten ersten Lasten auf der undeformierten Struktur sind in der Abbildung $81 \mathrm{zu}$ sehen. Die dargestellten Kräfte sind die über die entsprechenden Flächen der finiten Volumen aufintegrierten Drücke. In Abbildung 82 sind die auf die Strukturknoten transportierten Kräfte aus der ersten Strömungsberechnung dargestellt. Die Deformationen, die mit dem Strukturcode aus diesen Kräften berechnet werden, sind in Abbildung $83 \mathrm{zu}$ sehen. Die auf die Strömungsknoten interpolierten Deformationen zeigt die Abbildung 84.

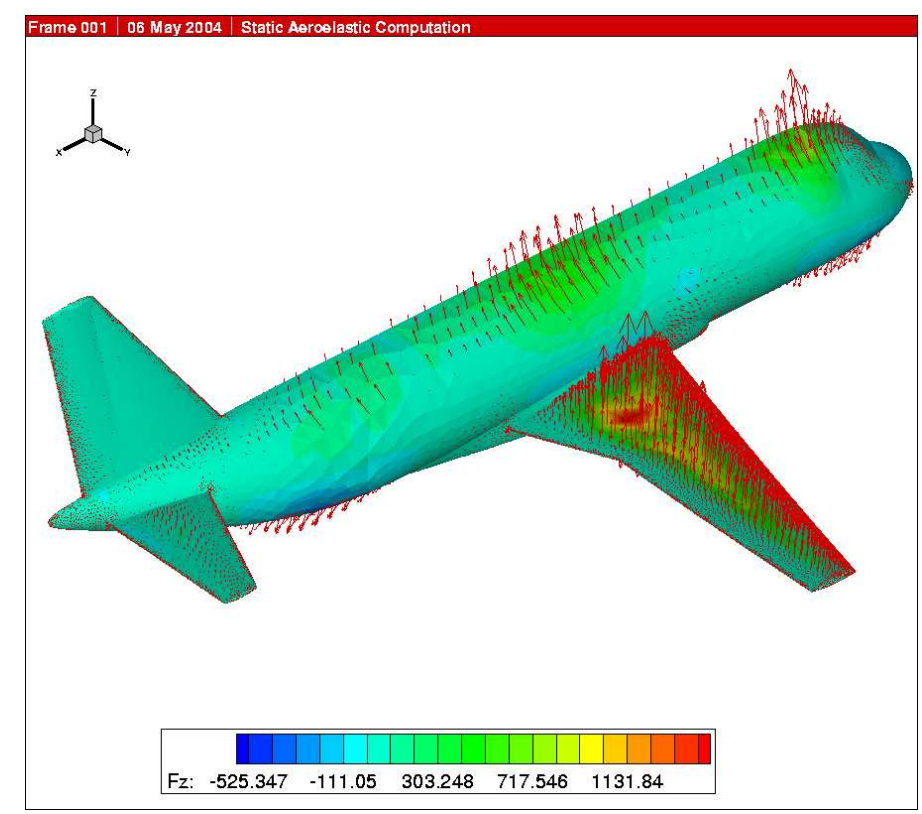

Abbildung 81. Die aufintegrierten Kräfte nach der ersten Strömungsrechnung. 


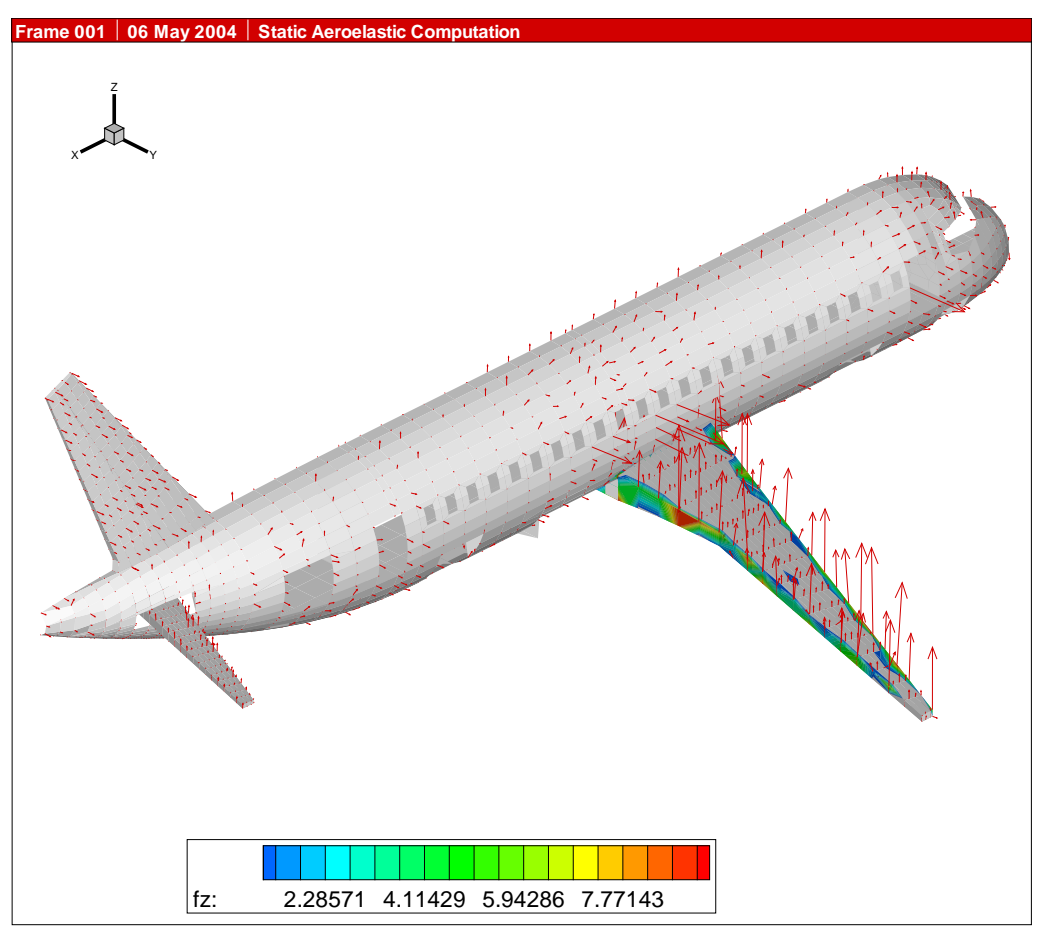

Abbildung 82. Die transportierten Kräfte auf den Strukturknoten.

Die im Folgenden präsentierten elastischen Deformationen auf der Struktur und die Druckverteilungen auf der Flugzeugoberfläche durch die Strömung zeigen die Endresultate des konvergierten Verfahrens.

Die maximalen Verformungen werden, wie in Abbildung $85 \mathrm{zu}$ sehen, an der Flügelspitze und am horizontalen Leitwerk berechnet. Der Einfluss der Verformung auf die Strömung wird an den Druckverteilungen auf der Flugzeugoberfläche in den Abbildungen 86 und 87 deutlich. In Abbildung 86 ist die Druckverteilung bei einem als starr angenommenen Flugzeug gezeigt, d.h. hier wurde die Strömung ohne den Einfluss der Wechselwirkung zur Struktur berechnet. Die mit der Stömung-Struktur-Kopplung berechnete Druckverteilung für ein reales, elastisches Flugzeug ist in Abbildung 87 dargestellt. Der Vergleich der beiden Abbildungen zeigt, dass der Einfluss der Wechselwirkung zwischen Strömung und Struktur nicht vernachlässigbar sondern von großer Bedeutung für eine realistische Simulation ist.

Testfälle. Wie schon zu Beginn des Abschnitts erläutert, werden in dieser gekoppelten Simulation zwei Interpolationsverfahren verglichen: Der neu entwickelte Algorithmus mit der „Partition of Unity“ Methode und das in vielen gekoppelten Simulationen bewährte Verfahren der globalen Interpolation mit radialen Basisfunktionen werden unter Verwendung verschiedener radialer Basisfunktionen gegenübergestellt. Zusätzlich wurde das Struktur- und Strömungsgitter manuell jeweils in die vier Komponenten Flügel, Rumpf, horizontales und vertikales Leitwerk (Höhen- und Seitenflosse) geteilt, wie in 


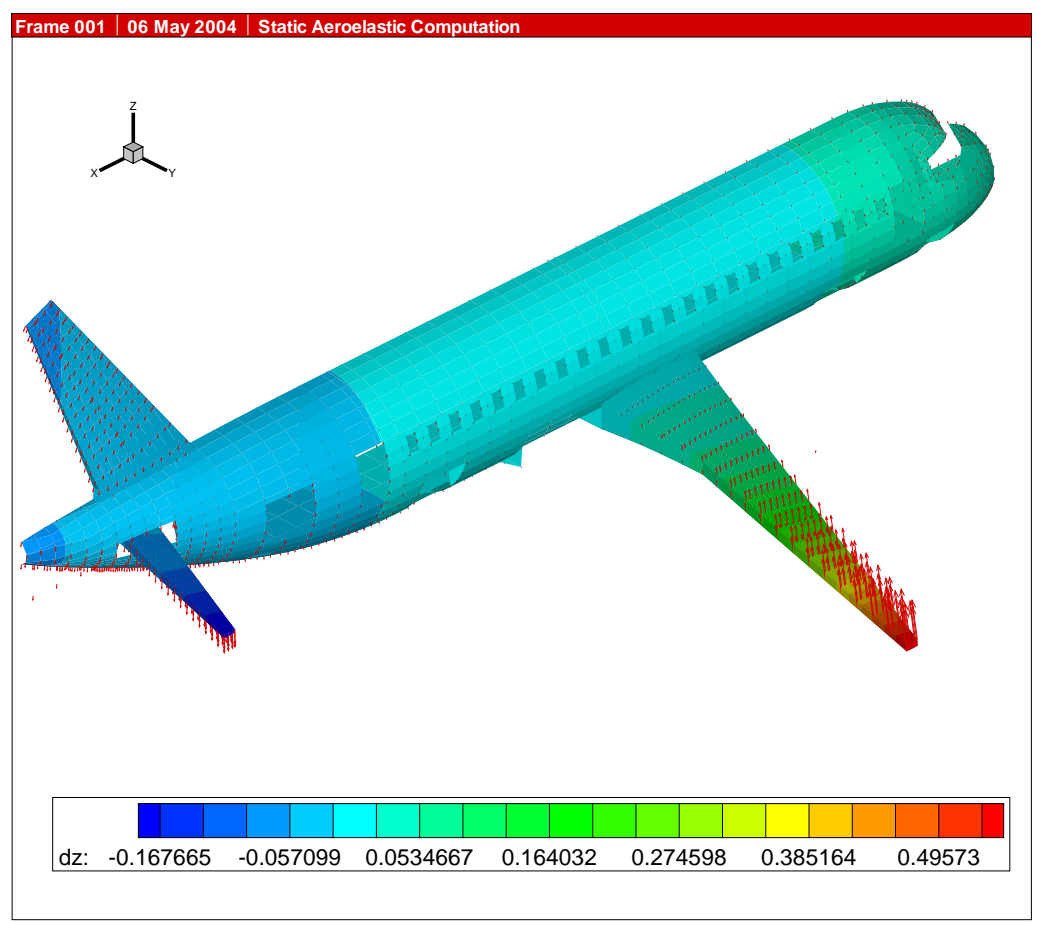

Abbildung 83. Im ersten Durchlauf des Iterationsverfahrens durch den Strukturcode berechnete Deformationen auf den Strukturknoten.

den Abbildungen 88 und 89 gezeigt. So wird die Kopplung in den verschiedenen Testkonfigurationen teilweise mit komponentenweiser Interpolation und teilweise mit Interpolation der kompletten Kopplungsgitter der Strömungsund Strukturseite als eine Komponente durchgeführt. Bei der komponentenweisen Interpolation werden die resultierenden Werte über Kompatibilitätsbedingungen für die Deformationen an den Schnittstellen der Komponenten zusammengefügt; trotzdem kann die komponentenweise Interpolation im Allgemeinen nicht so glatt sein wie die Interpolation unter Verwendung der kompletten Kopplungsgitter der Strömungs- und Strukturseite als eine Komponente. Die komponentenweise Interpolation ist eine Technik, die bei nur geringem zur Verfügung stehendem Speicherplatz und hohen Anzahlen von Struktur- und Strömungsknoten bei der globalen Anwendung der radialen Basisfunktionen genutzt wird, da sie den Vorteil der Reduzierung der Rechenzeit und des Speicherplatzbedarfs hat.

In Tabelle 15 werden die Testfälle mit dem Algorithmus, der radialen Basisfunktion, der Anzahl polynomialer Terme, dem Faktor für den Trägerradius und der Anzahl der Komponenten spezifiziert. Die Abkürzungen für den Algorithmus und die radiale Basisfunktion sind in Abschnitt 2.3 und in Tabelle 19 des Anhangs A definiert. 


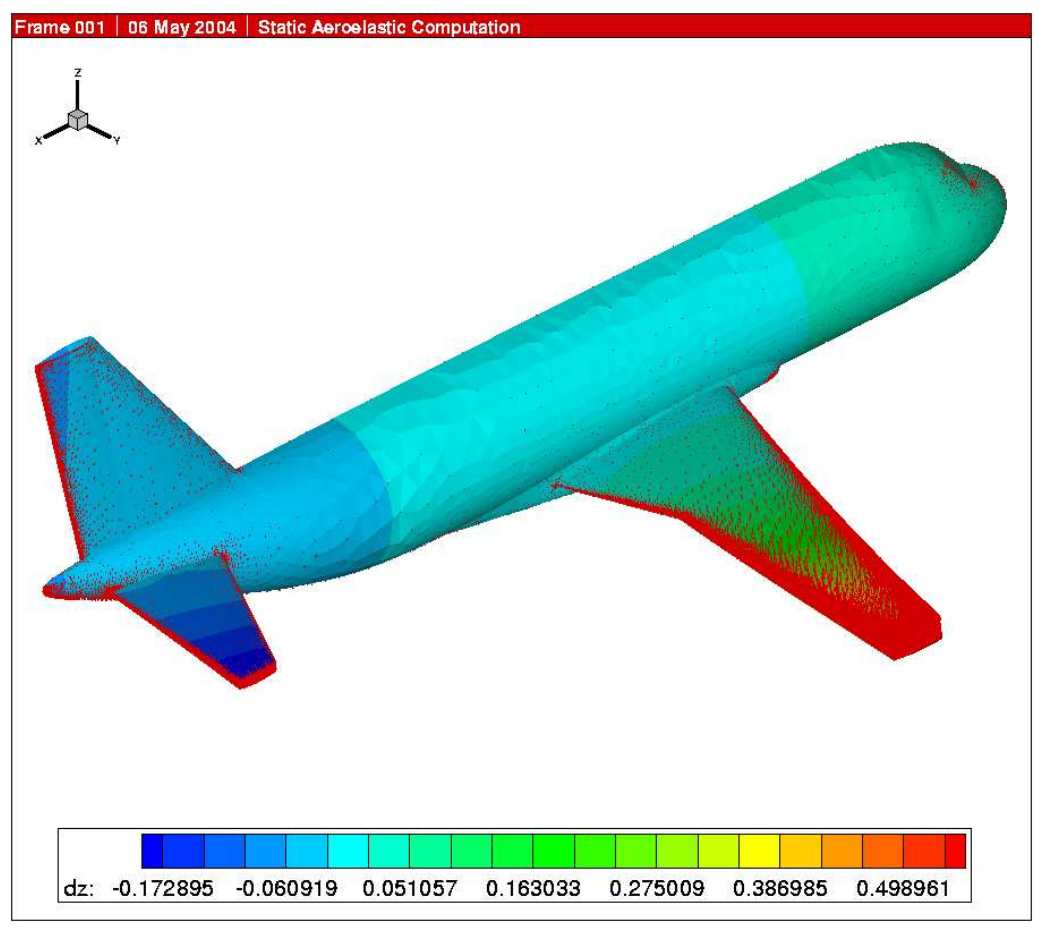

Abbildung 84. Die interpolierten Deformationen auf den Strömungsknoten.

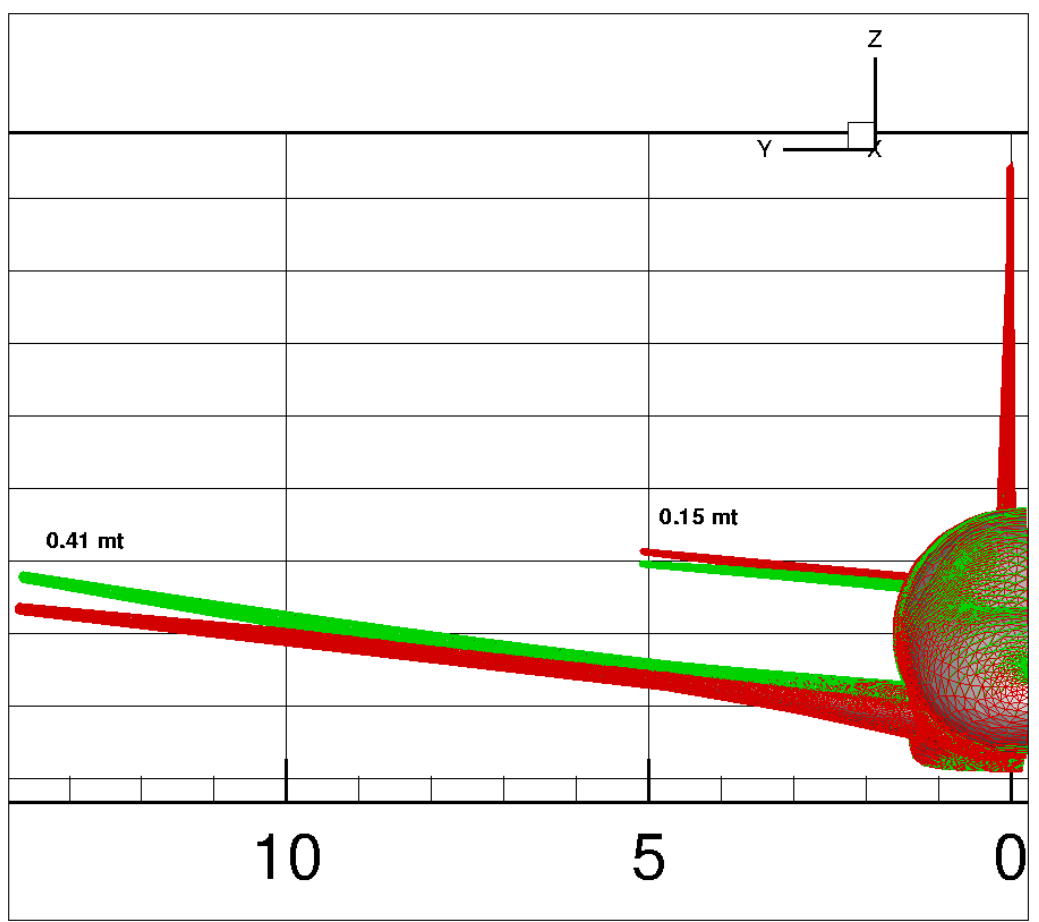

Abbildung 85. Die maximalen Verformungen am Flugzeug im aeroelastischen Gleichgewichtszustand. 


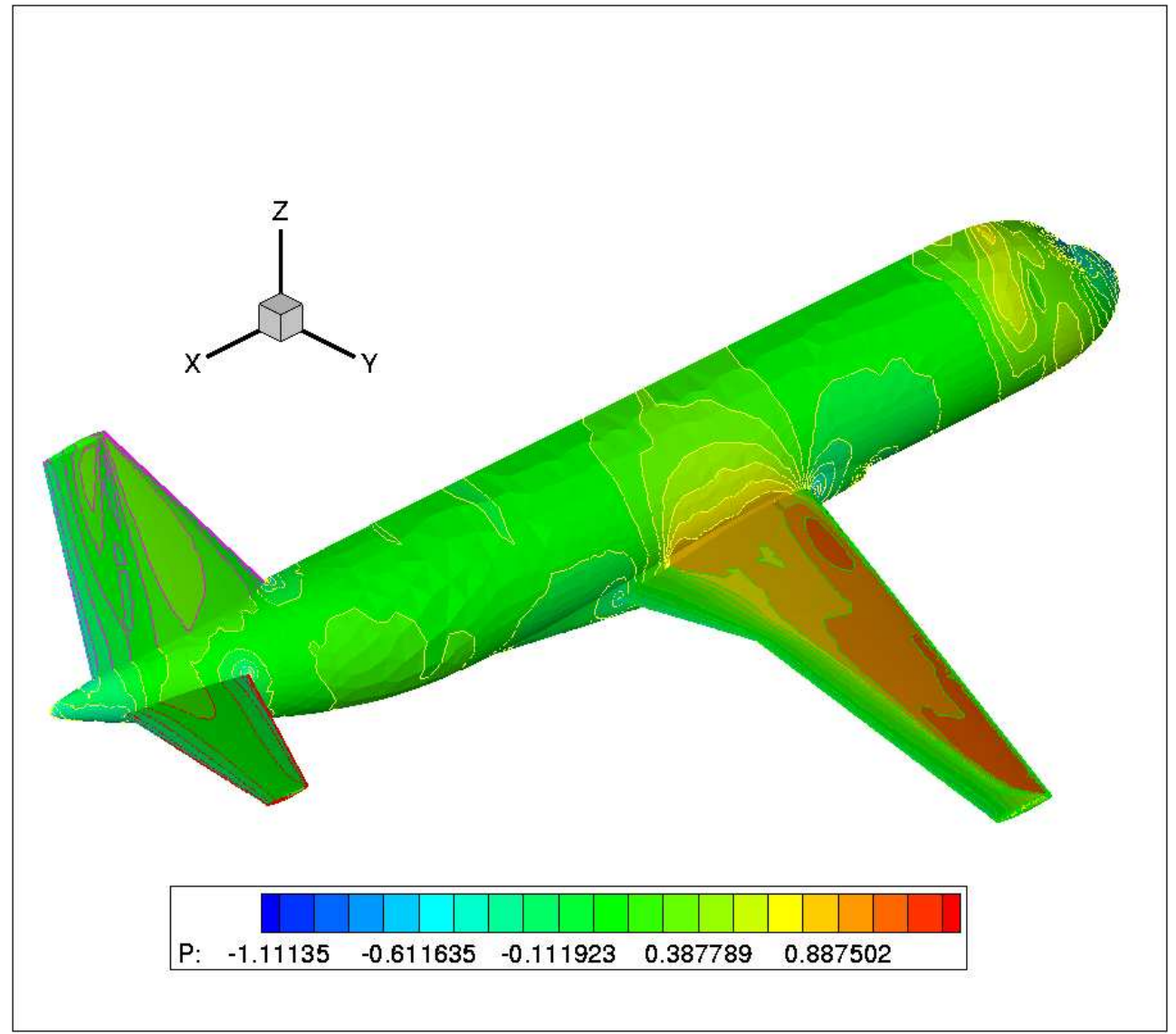

Abbildung 86. Die Druckverteilung an der Flugzeugoberfläche bei einem starren Flugzeug. 


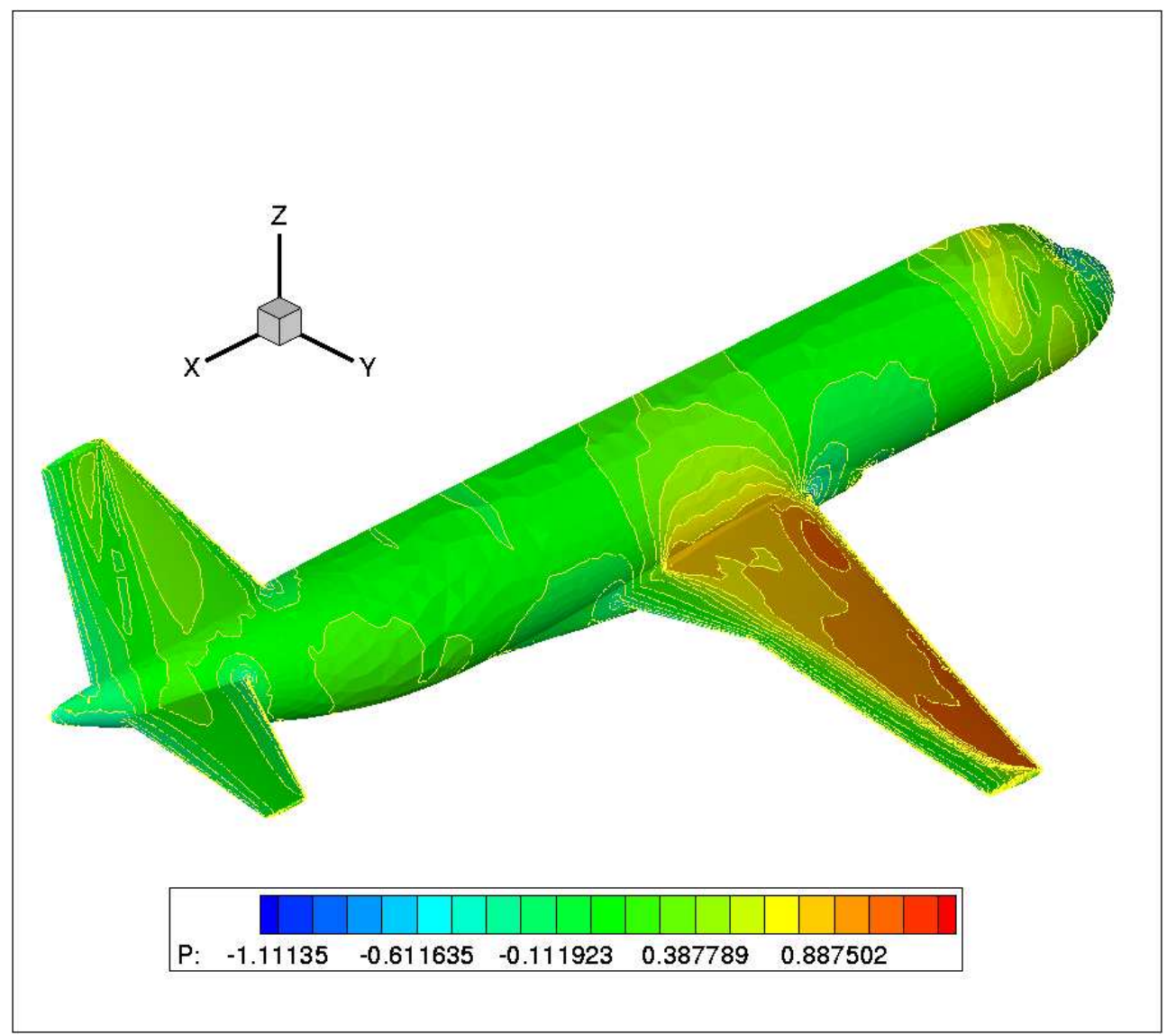

Abbildung 87. Die Druckverteilung im aeroelastischen Gleichgewichtszustand an der Flugzeugoberfläche bei einem realen, elastischen Flugzeug.

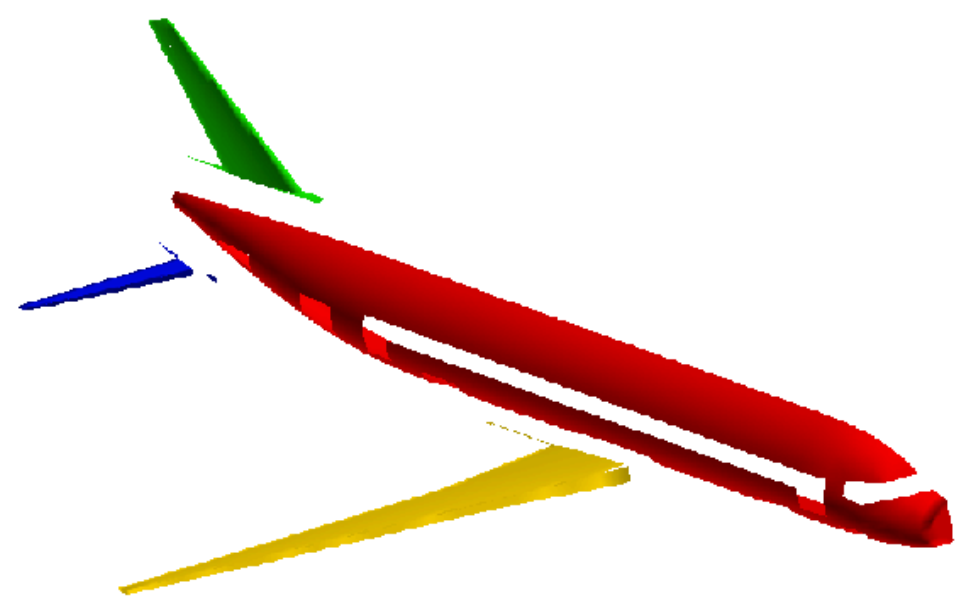

Abbildung 88. Die Aufteilung des Strukturmodells in Komponenten. 


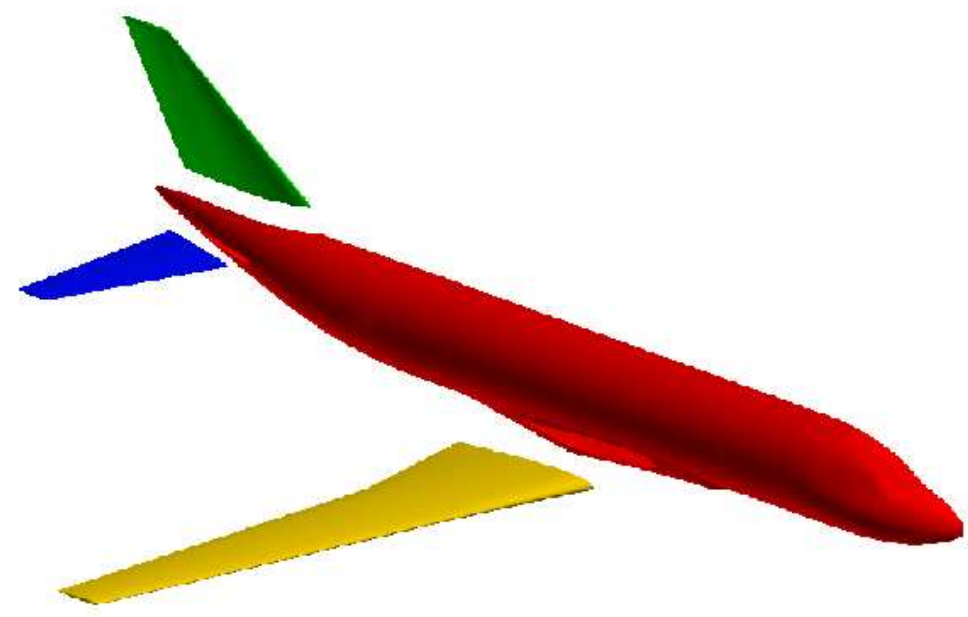

Abbildung 89. Die Aufteilung des Strömungsmodells in Komponenten.

\begin{tabular}{|c||c|c|c|c|c|}
\hline Testfall & Algorithmus & RBF & pT & Faktor & Anzahl der Komponenten \\
\hline 1 & GLOBAL/TRANS & $T P S$ & 4 & - & 4 \\
2 & GLOBAL/TRANS & $T P S$ & 4 & - & 1 \\
3 & POU/TRANS & $T P S$ & 4 & - & 4 \\
4 & GLOBAL/TRANS & $V S$ & 4 & - & 4 \\
5 & POU/TRANS & $V S$ & 4 & - & 4 \\
6 & POU/TRANS & $V S$ & 4 & - & 1 \\
7 & GLOBAL/TRANS & $E H$ & 4 & 1.0 & 4 \\
8 & POU/TRANS & $E H$ & 4 & 1.0 & 4 \\
9 & POU/TRANS & $E H$ & 4 & 1.0 & 1 \\
10 & GLOBAL/TRANS & $W 1$ & 4 & 1.0 & 4 \\
11 & GLOBAL/TRANS & $W 1$ & 4 & 100.0 & 4 \\
12 & POU/TRANS & $W 1$ & 4 & 1.0 & 4 \\
13 & POU/TRANS & $W 1$ & 4 & 1.0 & 1 \\
14 & POU/TRANS & $W 1$ & 4 & 100.0 & 1 \\
15 & GLOBAL/TRANS & $W 2$ & 4 & 1.0 & 4 \\
16 & POU/TRANS & $W 2$ & 4 & 1.0 & 4 \\
17 & POU/TRANS & $W 2$ & 4 & 1.0 & 1 \\
18 & POU/TRANS & $W 2$ & 4 & 100.0 & 1 \\
19 & GLOBAL/TRANS & $W 3$ & 4 & 1.0 & 4 \\
\hline
\end{tabular}

Tabelle 15. Die in der gekoppelten Simulation getesteten Konfigurationen. 


\subsection{Simulationsergebnisse}

Die Simulationsergebnisse werden zunächst unter Betrachtung der vertikalen Verschiebung entlang der Spannweite des Flügels, der Veränderung des Angriffswinkels und der Druckverteilung über die Flugzeugoberfläche analysiert.

Vertikale Verschiebung. Als erstes wird die Auswirkung der Wahl der radialen Basisfunktion bei globaler Anwendung und bei Verwendung der „Partition of Unity" Methode auf die vertikale Verschiebung entlang der Spannweite des Flügels untersucht. Die vertikale Verschiebung entlang der Spannweite des Flügels für die verschiedenen radialen Basisfunktionen bei globaler Interpolation und bei Anwendung der „Partition of Unity“ Methode ist in der Abbildung 90 dargestellt. Wie der Vergleich der Abbildungen zeigt, sind die Unterschiede in der Verschiebung bei Verwendung verschiedener radialer Basisfunktionen mit der „Partition of Unity“ Methode wesentlich geringer als bei der globalen Interpolation. Der maximale prozentuale Unterschied in der Verschiebung ist bei der globalen Interpolation 7.5 und bei der „Partition of Unity" Methode nur 0.5. Da die Wendland 3 Funktion eine Interpolante mit der höchsten Glattheit aber damit auch die am schlechtesten konditionierte Stützstellen-Matrix erzeugt, ist es erklärlich, dass bei dieser radialen Basisfunktion die größten Abweichungen auftreten.
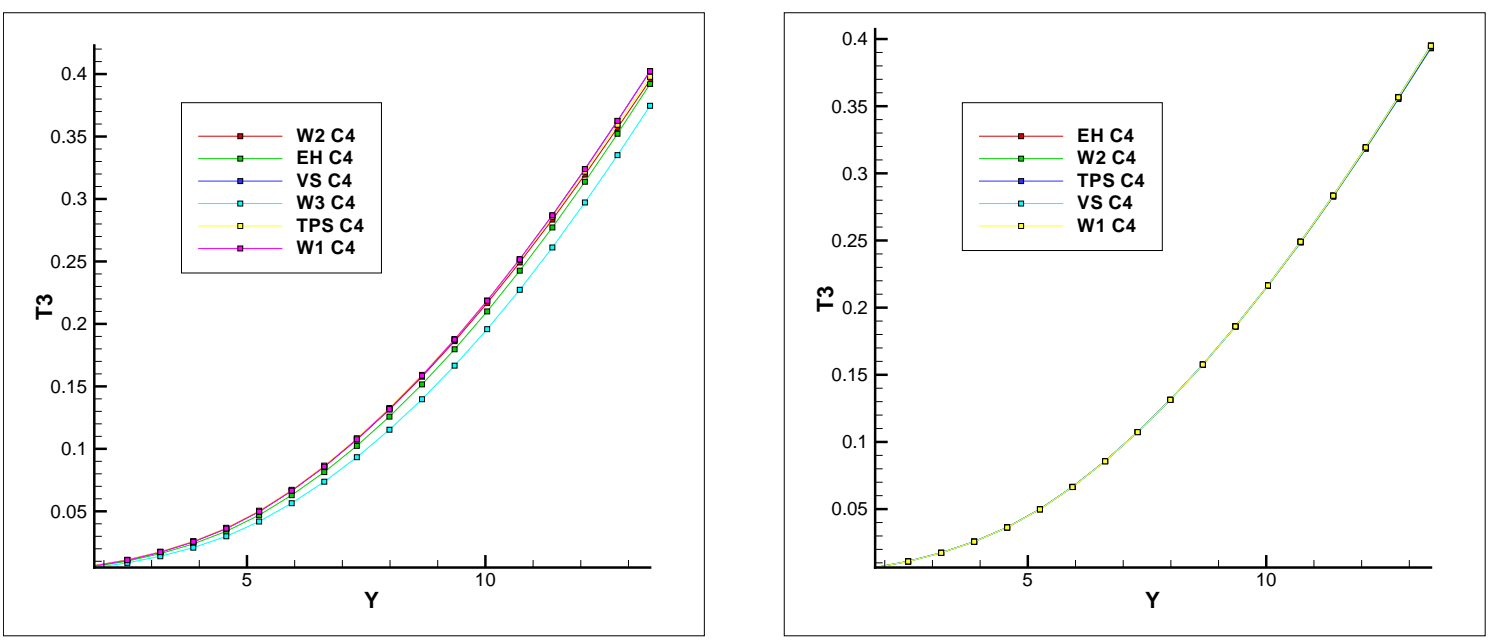

Abbildung 90. Die vertikale Verschiebung entlang der Spannweite des Flügels für die verschiedenen radialen Basisfunktionen. Die linke Grafik zeigt die Ergebnisse bei globaler Interpolation und die rechte Grafik bei Anwendung der „Partition of Unity" Methode.

Druckverteilung. Die gleiche Untersuchung für verschiedene radiale Basisfunktionen bei globaler Interpolation und der Verwendung der „Partition of 
Unity" Methode ist für die Druckverteilung durchgeführt worden. In Abbildung 91 sind die Druckverteilungen bei globaler Interpolation für die verschiedenen radialen Basisfunktionen zu sehen. Da die Druckverteilungen für die Wendland 2 und 3 Funktion nahezu identisch sind, wird die Druckverteilung für die Wendland 2 Funktion nicht gezeigt. Die Verteilungen, die über die Thin Plate Spline und die Wendland 3 (Wendland 2) Funktion berechnet worden sind, zeigen auf dem Flügel im Bereich der Wurzel größere Unterschiede. Weitere Abweichungen sind im Vergleich der Verteilungen zwischen der Wendland 1 und 3 Funktion zu sehen, die weniger glatte Interpolante der Wendland 1 Funktion erzeugt sogar isolierte Peaks mit relativ hohem Druck. Die Verteilung der Thin Plate Spline Funktion zeigt einen schmalen Streifen niedrigen Drucks entlang der Flügelachse von der Mitte der Spannweite des Flügels bis zur Spitze, der in den Druckverteilungen der anderen radialen Basisfunktionen nicht zu erkennen ist. Bei Verwendung der "Partition of Unity“ Methode ist dieser Streifen niedrigen Drucks bei allen verwendeten radialen Basisfunktionen vorhanden. Dieser Streifen ist theoretisch interpretierbar als ein Bereich lokaler Beschleunigung, den die Strömungspartikel nach dem schwachen Stoß nahe der Vorderkante erfahren. Wie in Abbildung $92 \mathrm{zu}$ sehen, stimmen auch die Druckverteilungen bei den verschiedenen radialen Basisfunktionen unter Anwendung der „Partition of Unity“ Methode besser überein als bei der globalen Interpolation.

Angriffswinkel. In der dritten Analysereihe wird die durch die Deformation hervorgerufene Torsion des Flügels untersucht. Die Torsion wird an Hand des Angriffswinkels gemessen, welcher durch eine lineare Interpolation der vertikalen Verschiebungen zwischen Punkten auf der Vorder- und Hinterkante des Flügels bestimmt wird. In Abbildung 93 sind die Angriffswinkel entlang der Spannweite des Flügels abgetragen für verschiedene radiale Basisfunktionen bei der globalen Interpolation und bei Anwendung der „Partition of Unity" Methode. Es zeigt sich in Abbildung 93, dass die Torsion am sensibelsten auf die Veränderung der radialen Basisfunktion bei globaler Interpolation reagiert. Die Abweichungen des Angriffswinkels bei der Wendland 2 und 3 Funktion von den berechneten Winkeln für die anderen radialen Basisfunktionen ist sogar erheblich. Die Betrachtung des graphischen Verlaufs des entlang der Spannweite des Flügels aufgetragenen Angriffswinkels zeigt, dass die Kurven für die Wendland 1 und 2 Funktion nicht nur deutliche Abweichungen zeigen, sondern auch einen qualitativ anderen Verlauf haben. Im Gegensatz zu den anderen Kurven wechselt bei diesen Kurven die Steigung von der Flügelwurzel bis zur Flügelspitze bei einer Spannweite von ca. 10m das Vorzeichen, welches eine andere Kurvengestalt impliziert. Im Gegensatz dazu liegen die Kurven des Angriffswinkels für verschiedene radiale Basisfunktionen bei der „Partition of Unity“ Methode nahezu übereinander, wie in Abbildung 93 dargestellt. 
Deformation des Flügelprofils. Ein weiterer Vergleich zwischen globaler Interpolation und Anwendung der "Partition of Unity“ Methode wird für die Deformation des Flügelprofils entlang verschiedener Schnitte durch den Flügel durchgeführt. Die vertikalen Verschiebungen werden für Schnitte durch den Flügel bei 0.18, 0.5, 0.75 und 0.95 der Spannweite gegen die Querrichtung des Flügels abgetragen. Diese Veränderung der Deformationen auf den Querschnitten durch den Flügel erreichen nur Bruchteile von Millimetern und sind in einer dreidimensionalen Darstellung des Flügels nur bei erheblicher Vergrößerung zu erkennen. Die im Folgenden diskutierten Unterschiede zwischen den Berechnungsergebnissen sind dementsprechend nur sehr gering. In den Abbildungen 94 bis 95 sind Deformationen über den Querschnitt gezeigt, die über die globale Interpolation mit radialen Basisfunktionen erzielt worden sind. Auf den Schnitten bei 0.18 der Spannweite finden sich die maximalen vertikalen Verschiebungen nicht an der Hinterkante, sondern einen kurzen Abschnitt vorher. Dieses Phänomen tritt typischerweise bei Kopplungen der gesamten Strömungs- und Strukturgitter der Kopplungsfläche mit der globalen Interpolation auf Schnitten bei 0.18 und 0.95 der Spannweite auf. Dies ist aber gegensätzlich zur Konstruktion der Flügel, die über die Steifigkeit der Rippen die Form des Querschnitts starr halten soll. In den Abbildungen 96 bis 101, die die Verschiebungen zeigen, die über die globale Interpolation bei komponentenweiser Kopplung und über die „Partition of Unity“ Methode berechnet worden sind, ist die Querschnittsform fast unverändert. Damit liegt der Schluss nahe, dass die Veränderung der Querschnittsform durch unerwünschte Einflüsse von Werten an anderen Flugzeugteilen in der globalen Interpolation hervorgerufen werden.

Transformation der Kräfte. Die Transformation der Kräfte durch die Transponierte der Kopplungsmatrix zeigt einen weiteren Vorteil der „Partition of Unity" Methode gegenüber der globalen Interpolation mit radialen Basisfunktionen. Wie im Vergleich der Abbildungen 102 und $103 \mathrm{zu}$ sehen ist, sind die hohen Kräfte unter Verwendung der "Partition of Unity“ Methode gleichmäßiger auf die Vorder- und Hinterkante des Flügel-Strukturmodells verteilt, als bei Verwendung der globalen Interpolation. Die Transformation der Kräfte durch die Transponierte der über die „Partition of Unity“ Methode berechneten Kopplungsmatrix entspricht mehr einer physikalisch sinnvollen Verteilung als bei dem entsprechenden Verfahren über die globale Interpolation. Die Ursache hierfür liegt in der lokalen Verteilung der Kräfte bei der „Partition of Unity“ Methode. Durch die Verwendung der Transponierten wird, wie in Kapitel II Abschnitt 2.2 gezeigt, die Gesamtsumme der Kräfte bei beiden Methoden erhalten; durch die „Partition of Unity“ Methode wird aber zusätzlich die Gesamtsumme der gewichteten Kräfte in einer Partition erhalten. So werden die Kräfte der Strömungsseite nur innerhalb der Partitionen auf die Strukturknoten verteilt, in denen sie jeweils liegen.

Ein weiterer Vorteil resultiert aus der oben diskutierten gleichmäßigeren Verteilung der Kräfte besonders bei Verwendung der „Partition of Unity“ Me- 
thode mit einer Wendland Funktion. Durch die so verteilten Kräfte wird auch die im Strukturcode berechnete Deformation so glatt, dass der Strömungscode bei dieser Verformung um 10 Prozent schneller zu einer Lösung kommt als bei Verwendung der globalen Interpolation.

Vergleichsdaten. Da keine experimentellen Vergleichsdaten für die Ergebnisse der hier durchgeführten Simulation vorhanden sind, werden die Resultate mit Daten verglichen, die aus Simulationen stammen, die mit anderen Tools berechnet worden sind. Diese Simulationen wurden ebenfalls von Mitgliedern des TAURUS Projekts durchgeführt. Die Vergleichsdaten sind mit dem kommerziellen Finite Elemente Programm MSC.NASTRAN und einer räumlichen Interpolation zwischen Struktur- und Strömungsfeld über die „Surface Spline" Methode berechnet worden. Der Strukturcode MSC.NASTRAN eignet sich besonders zur Validierung der Ergebnisse, da er eines der bekanntesten im industriellen Produktionsprozess eingesetzten Finite Elemente Programme ist. Da zur Berechnung komplett andere Verfahren eingesetzt werden, wird der Vergleich an Hand von globalen Größen durchgeführt. Der Vergleich der aerodynamischen Koeffizienten, der maximalen vertikalen Verschiebung und der entlang der Spannweite des Flügels abgetragenen vertikalen Verschiebungen ist in Tabelle 16 sowie in Abbildung 104 gezeigt.

\begin{tabular}{|c|c|c|c|}
\hline & $\mathrm{Cl}$ & $\mathrm{Cd}$ & $\mathrm{Max}$ T3[m] \\
\hline MSC.NASTRAN & 0.443 & 0.0373 & 0.505 \\
\hline $\begin{array}{c}\text { Kopplung über } \\
\text { „Partition of Unity“ }\end{array}$ & 0.431 & 0.0303 & 0.432 \\
\hline
\end{tabular}

Tabelle 16. Vergleich der aerodynamischen Koeffizienten und der maximalen vertikalen Verschiebung.

Unter Berücksichtigung der komplett unterschiedlichen Kopplungsmethoden und der verwendeten linearen aerodynamischen Theorie bei MSC.NASTRAN, mit der das komplexe Feld einer transsonischen Strömung nicht geeignet beschrieben werden kann, zeigen die Ergebnisse eine zufriedenstellende Übereinstimmung.

Rechenzeiten. Die über MpCCI (siehe Kapitel III Abschnitt 1) gekoppelte Simulation wird auf einem Linux Workstation Cluster mit 8 Xeon $2.66 \mathrm{GHz}$ Prozessoren und 2 bis 4 Gbyte RAM durchgeführt. Da der Strömungscode TAU die meiste Rechenzeit benötigt, wird der Aerodynamik Code auf 6 Prozessoren gestartet. Beide, der Strukturcode und der Aeroelastik-Code, der Kopplungsaufgaben realisiert, laufen jeweils auf einem Prozessor. 
Die Rechenzeiten sind für eine gekoppelte Simulation von entscheidender Bedeutung, insofern sind die Vergleiche zwischen den verschiedenen Testkonfigurationen bezüglich der Rechenzeiten ein Teil der wichtigsten Ergebnisse dieser Arbeit. Wie in Tabelle $17 \mathrm{zu}$ sehen ist, zeigt bei der globalen Anwendung der radialen Basisfunktionen die Wendland 1 Funktion die niedrigsten Rechenzeiten. Die Funktionen mit kompaktem Träger haben hinsichtlich der Rechenzeiten den Vorteil der dünn besetzten Struktur der Stützstellenmatrix. Dieser Vorteil resultiert aber bei einer schlechteren Konvergenz der Matrix nicht unbedingt in einer niedrigeren Rechenzeit, wie Tabelle 17 zeigt.

Entscheidender für diese Arbeit ist aber der Vergleich der Rechenzeiten bei der globalen Interpolation mit radialen Basisfunktionen und bei der Anwendung der "Partition of Unity“ Methode. Wie bereits in Kapitel II Abschnitt 3.1 ausführlich beschrieben, ist die globale Anwendung der radialen Basisfunktionen bei hohen Anzahlen von Stützstellen sehr teuer, so zeigt sich auch im Vergleich in der Tabelle 18, dass der Rechenzeitvorteil bei der „Partition of Unity" Methode gegenüber der globalen Anwendung der radialen Basisfunktionen bei steigender Anzahl von Stützstellen stark wächst, welches der Theorie in Kapitel II Abschnitt 3 entspricht. Die Rechenzeiten zur Aufstellung der Kopplungsmatrizen in der Tabelle 18 sind unter Verwendung der Wendland 2 Funktion gemessen worden.

\begin{tabular}{|c||c|c|c|c|c|c|}
\hline Radiale Basisfunktion & TPS & VS & EH & W1 & W2 & W3 \\
\hline Rechenzeit in Sekunden & 450 & 576 & 593 & 327 & 514 & 500 \\
\hline
\end{tabular}

Tabelle 17. Die Rechenzeiten für die Aufstellung der Kopplungsmatrizen bei der globalen Anwendung der verschiedenen radialen Basisfunktionen. Die Interpolation wurde komponentenweise durchgeführt mit der Aufteilung der Kopplungsgitter von Strömungs- und Strukturseite in 4 Komponenten.

\begin{tabular}{|c||c|c|}
\hline Algorithmus & Anzahl der Komponenten & Rechenzeit in Sekunden \\
\hline GLOBAL/TRANS & 1 & 5000 \\
POU/TRANS & 1 & 600 \\
GLOBAL/TRANS & 4 & 514 \\
POU/TRANS & 4 & 210 \\
\hline
\end{tabular}

Tabelle 18. Die Rechenzeiten für die Aufstellung der Kopplungsmatrizen bei der globalen Interpolation mit radialen Basisfunktionen und mit der „Partition of Unity" Methode. 

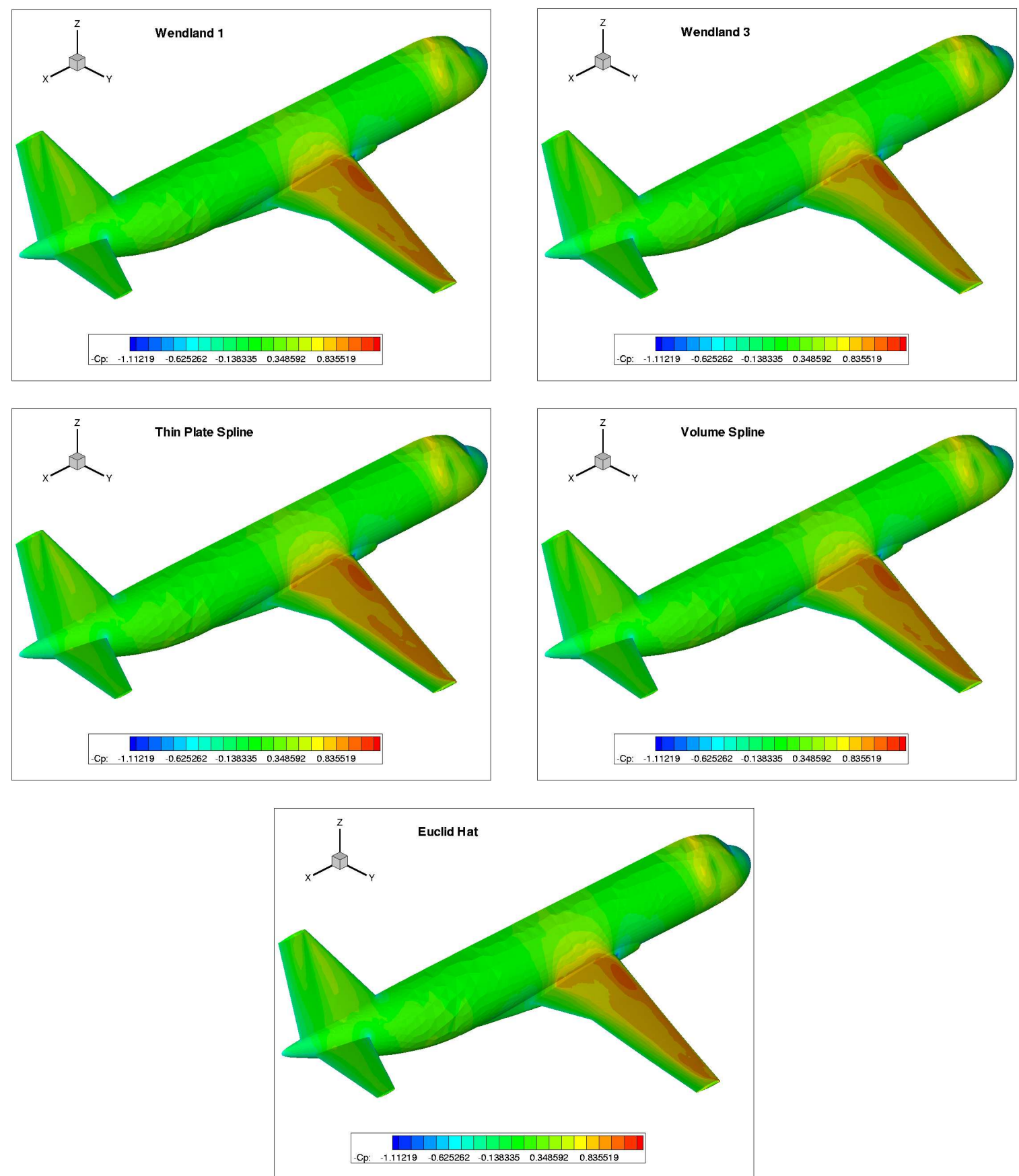

Abbildung 91. Die Druckverteilung bei verschiedenen radialen Basisfunktionen unter Verwendung der globalen Interpolation. 

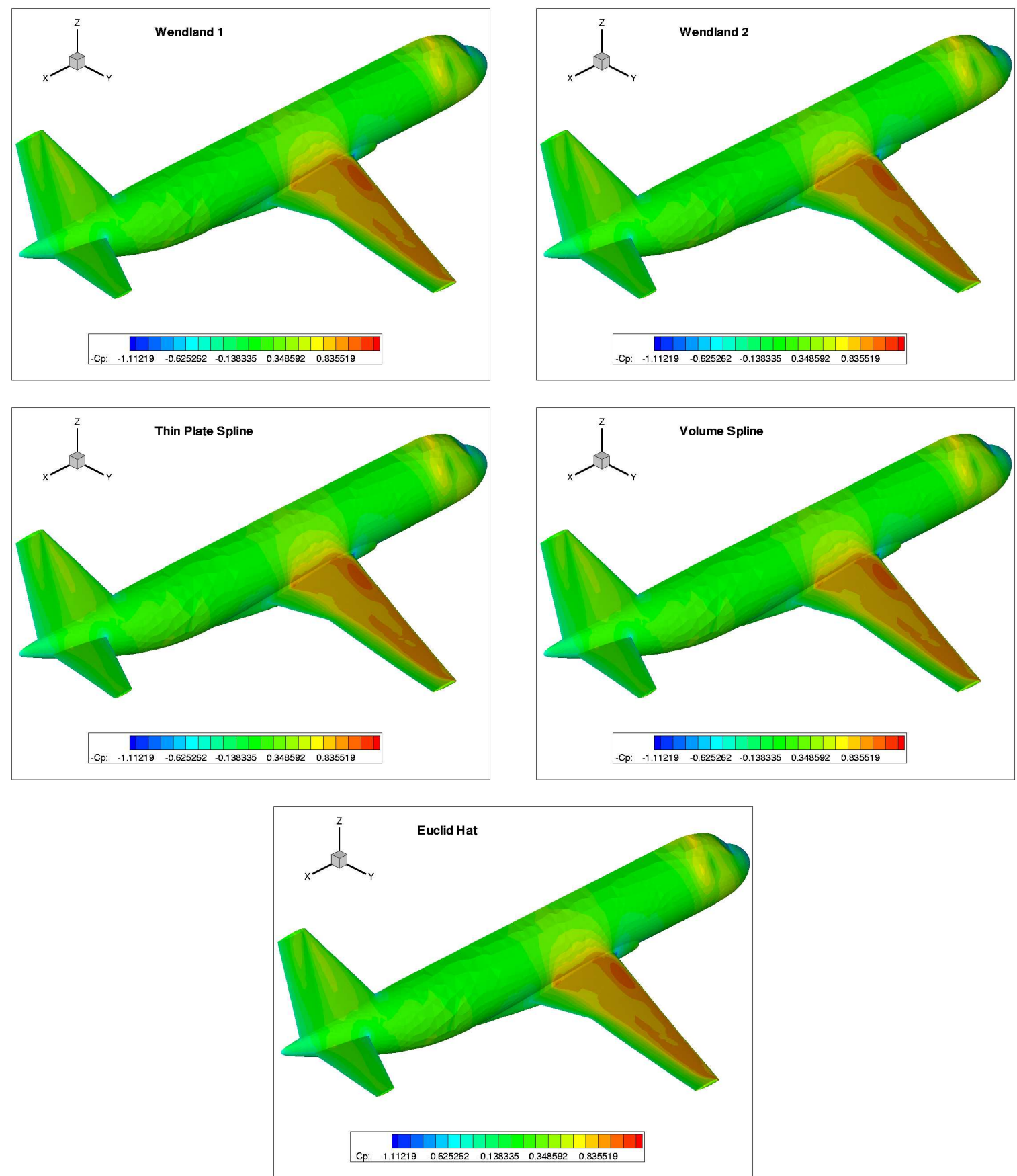

Abbildung 92. Die Druckverteilung bei verschiedenen radialen Basisfunktionen unter Verwendung der „Partition of Unity“ Methode. 

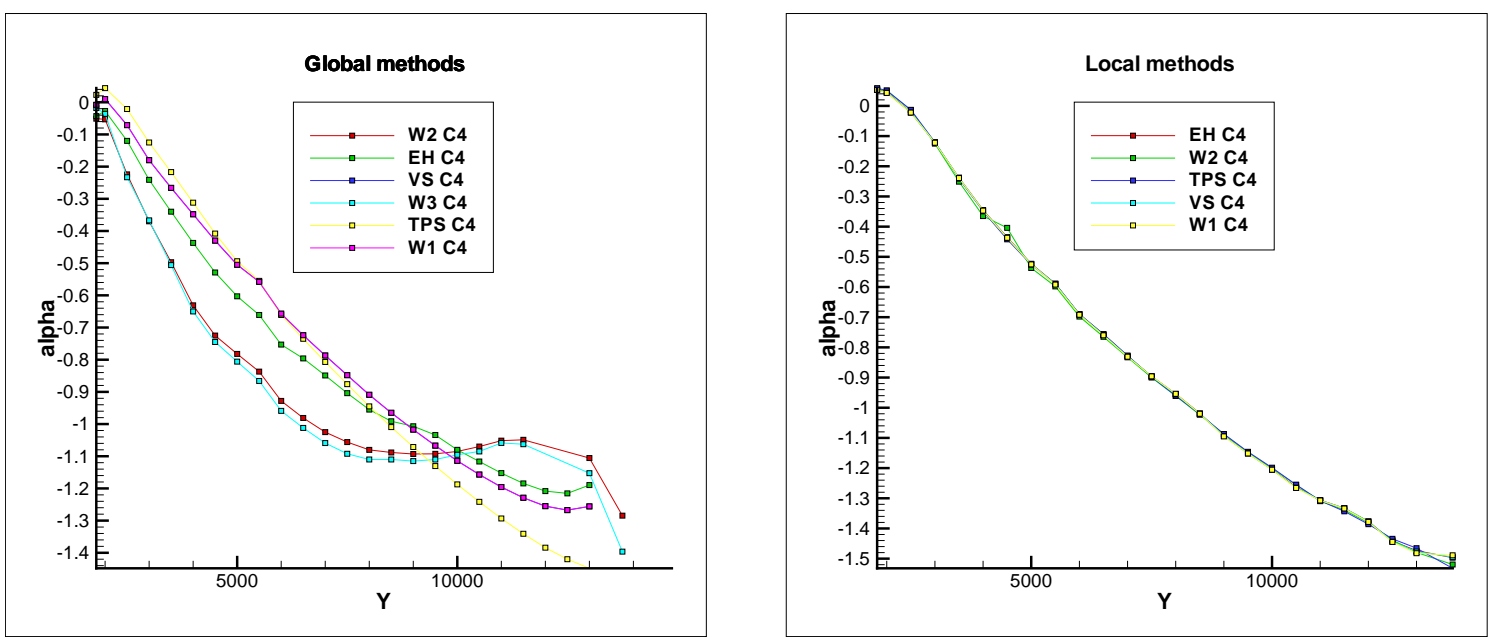

Abbildung 93. Der Angriffswinkel entlang der Spannweite des Flügels abgetragen für verschiedene radiale Basisfunktionen auf der linken Grafik mit globaler Interpolation und auf der rechten Grafik bei Anwendung der "Partition of Unity“ Methode.
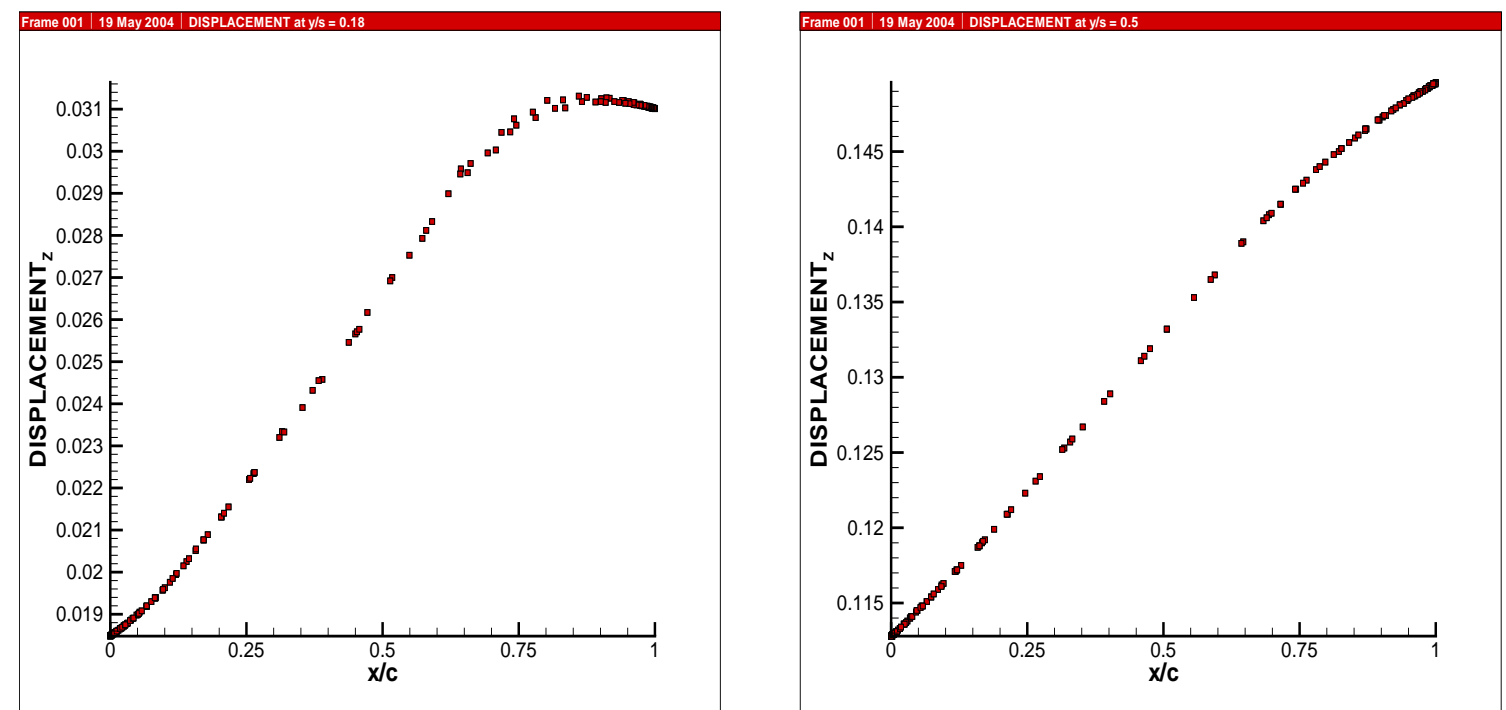

Abbildung 94. Die vertikale Verschiebung auf dem Flügelquerschnitt bei globaler Interpolation über die kompletten Kopplungsgitter mit der Thin Plate Spline Funktion. 

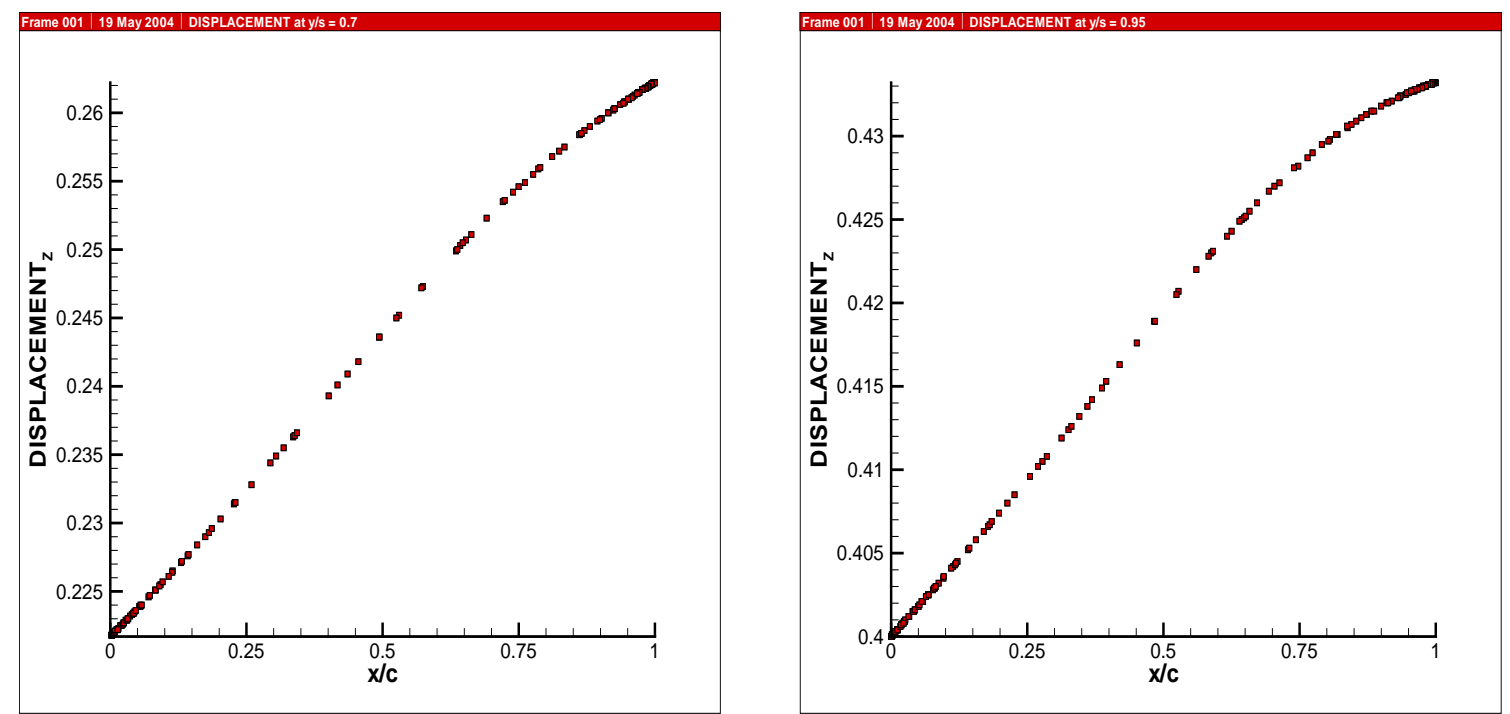

Abbildung 95. Die vertikale Verschiebung auf dem Flügelquerschnitt bei globaler Interpolation über die kompletten Kopplungsgitter mit der Thin Plate Spline Funktion.
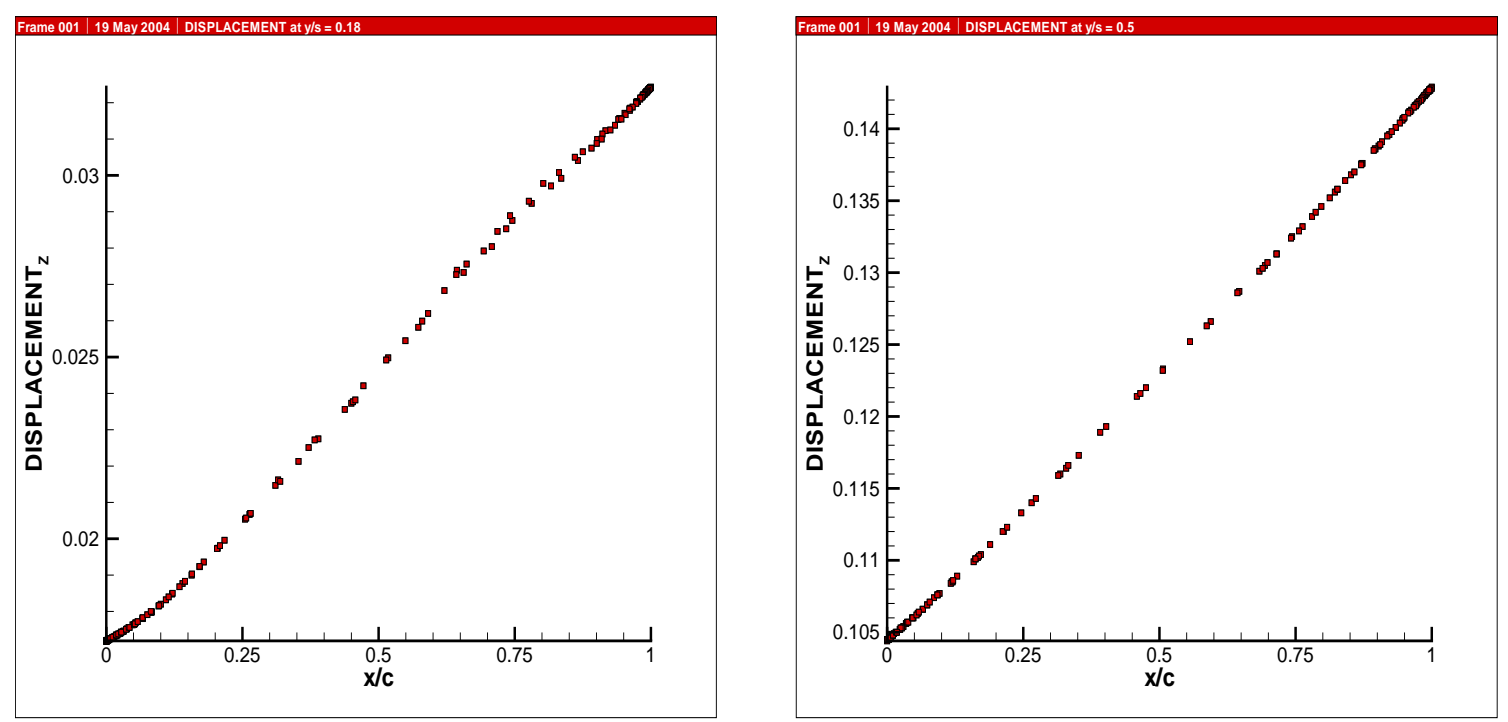

Abbildung 96. Die vertikale Verschiebung auf dem Flügelquerschnitt bei komponentenweiser globaler Interpolation mit der Thin Plate Spline Funktion. 

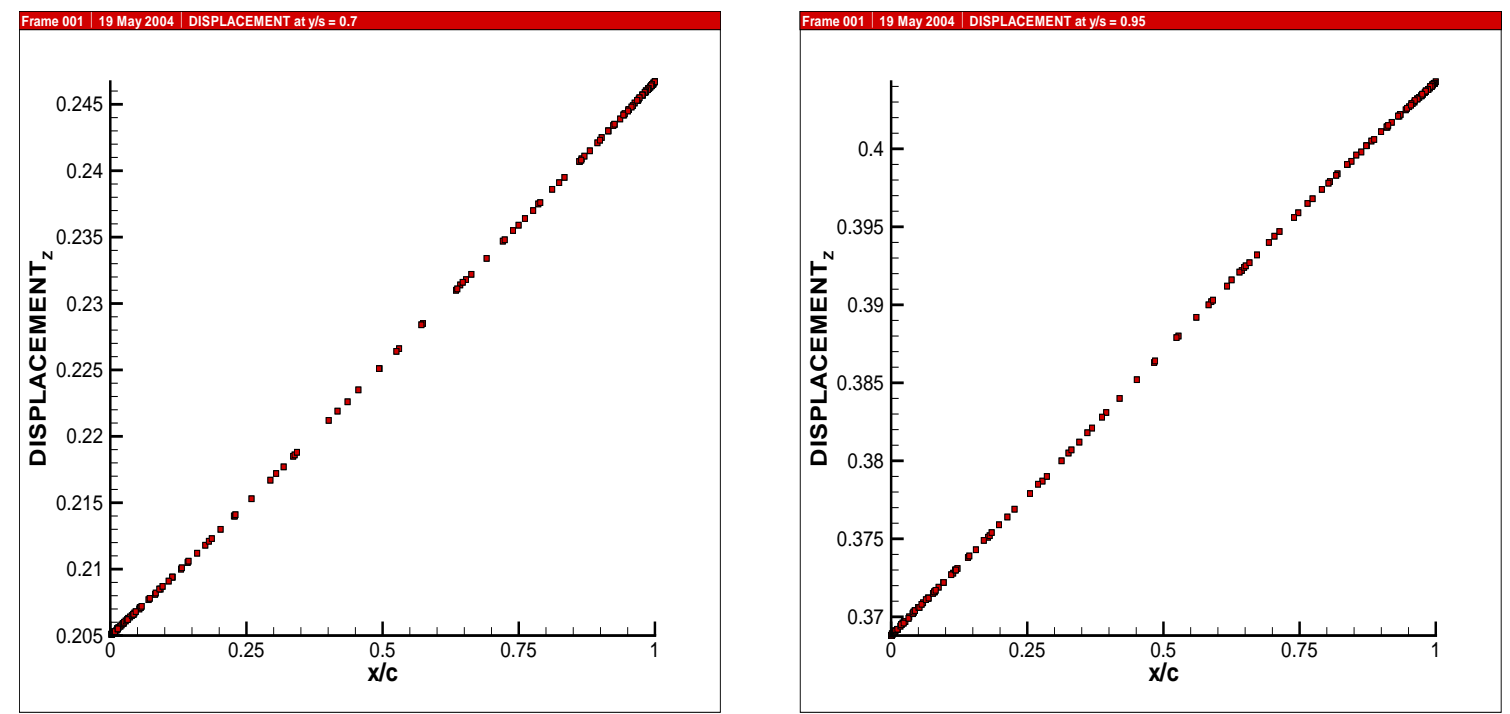

Abbildung 97. Die vertikale Verschiebung auf dem Flügelquerschnitt bei komponentenweiser globaler Interpolation mit der Thin Plate Spline Funktion.
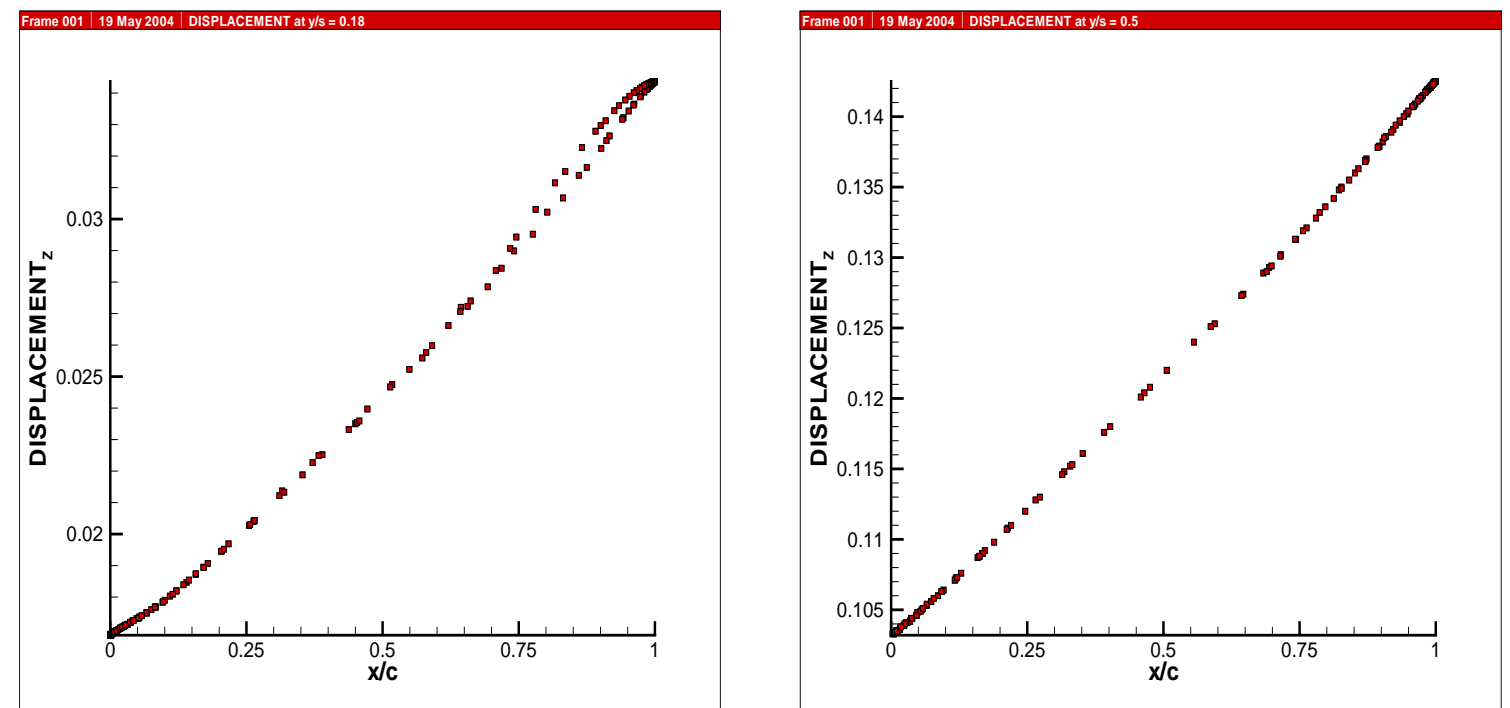

Abbildung 98. Die vertikale Verschiebung auf dem Flügelquerschnitt bei komponentenweiser Interpolation über die „Partition of Unity“ Methode mit der Wendland 1 Funktion. 

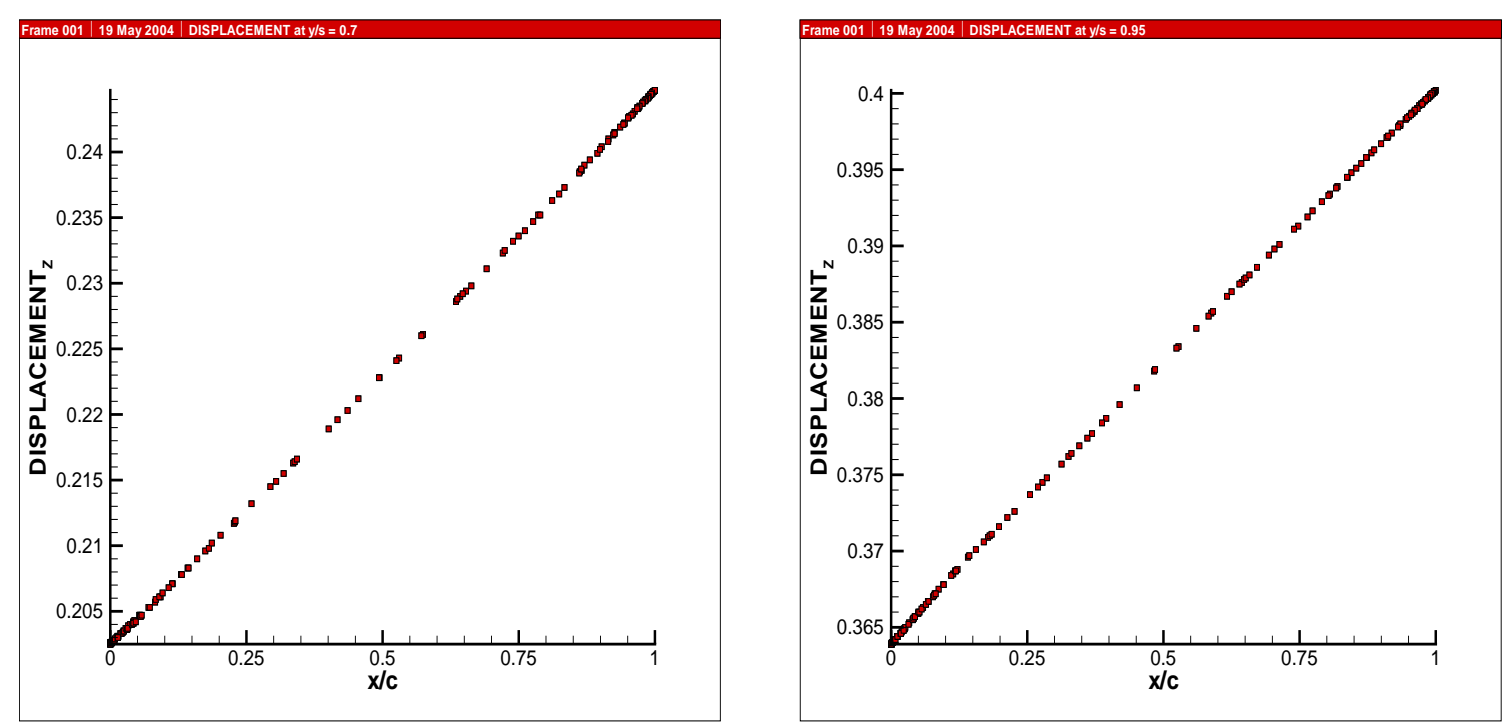

Abbildung 99. Die vertikale Verschiebung auf dem Flügelquerschnitt bei komponentenweiser Interpolation über die „Partition of Unity“ Methode mit der Wendland 1 Funktion.
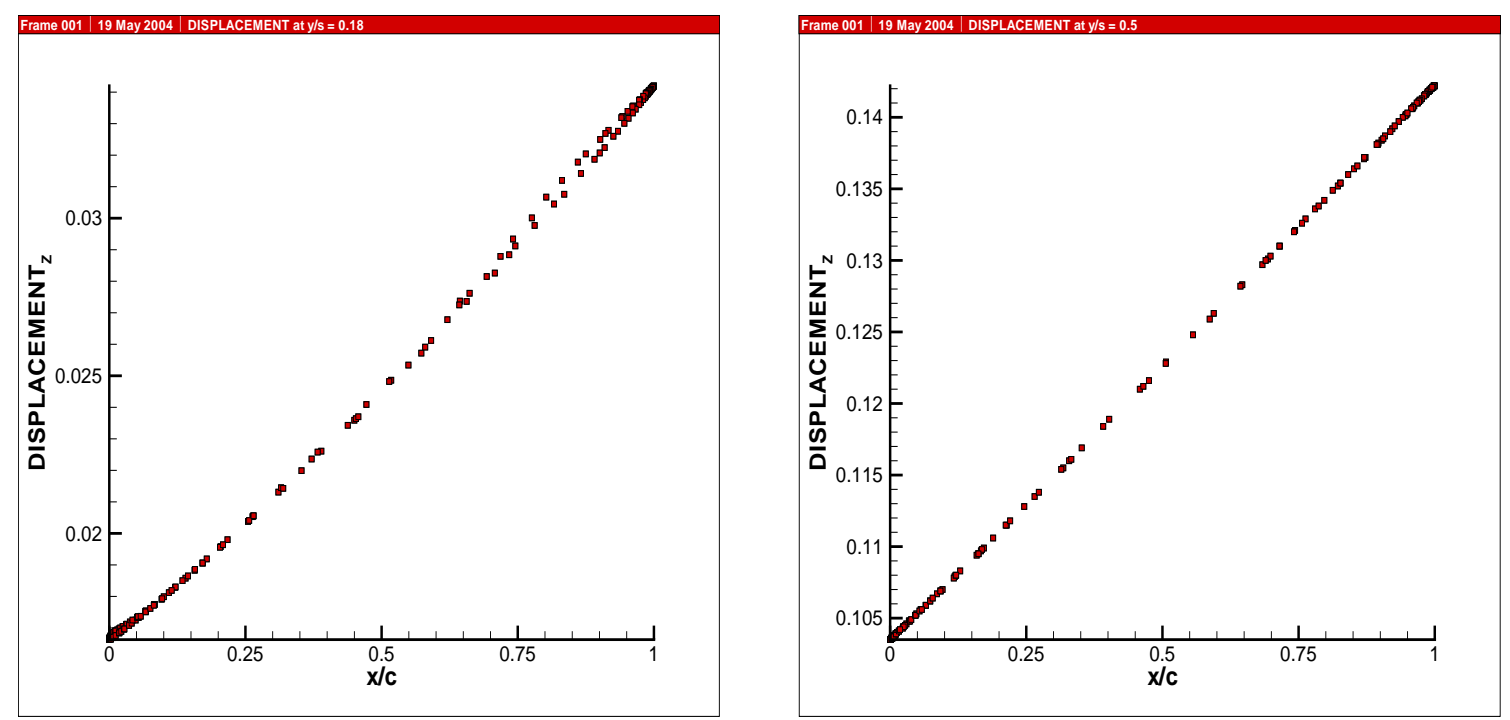

Abbildung 100. Die vertikale Verschiebung auf dem Flügelquerschnitt bei Interpolation der gesamten Kopplungsfläche über die „Partition of Unity“ Methode mit der Wendland 1 Funktion. 

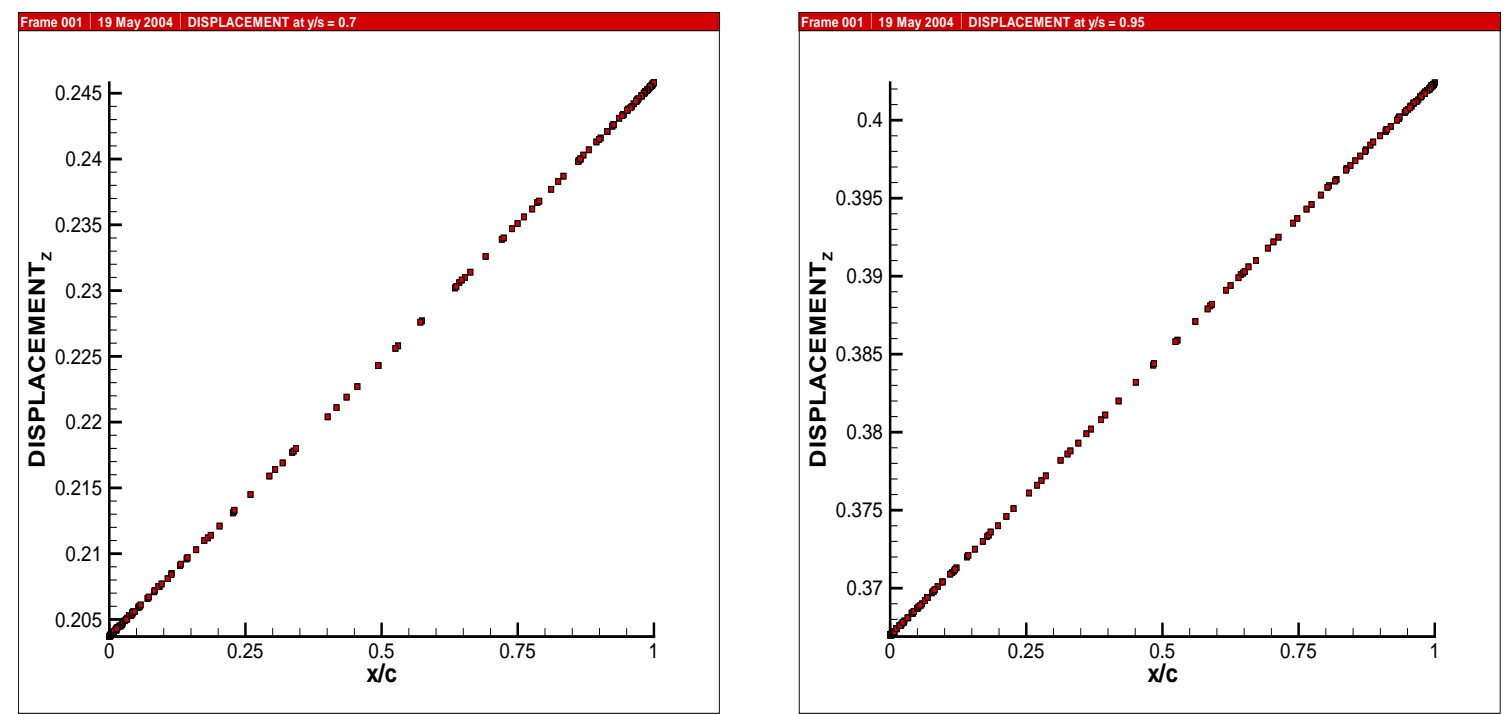

Abbildung 101. Die vertikale Verschiebung auf dem Flügelquerschnitt bei Interpolation der gesamten Kopplungsfläche über die „Partition of Unity“ Methode mit der Wendland 1 Funktion.
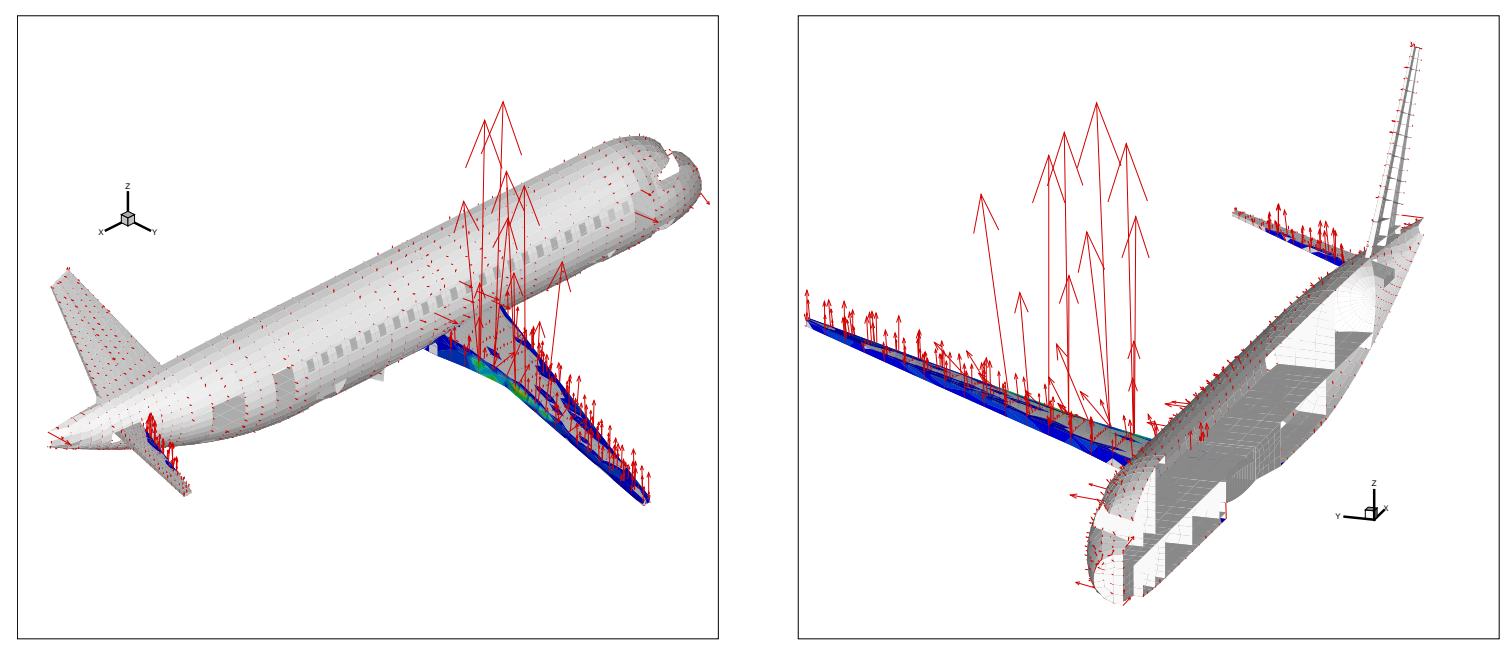

Abbildung 102. Die transformierten Kräfte auf dem Strukturmodell bei globaler Interpolation. 

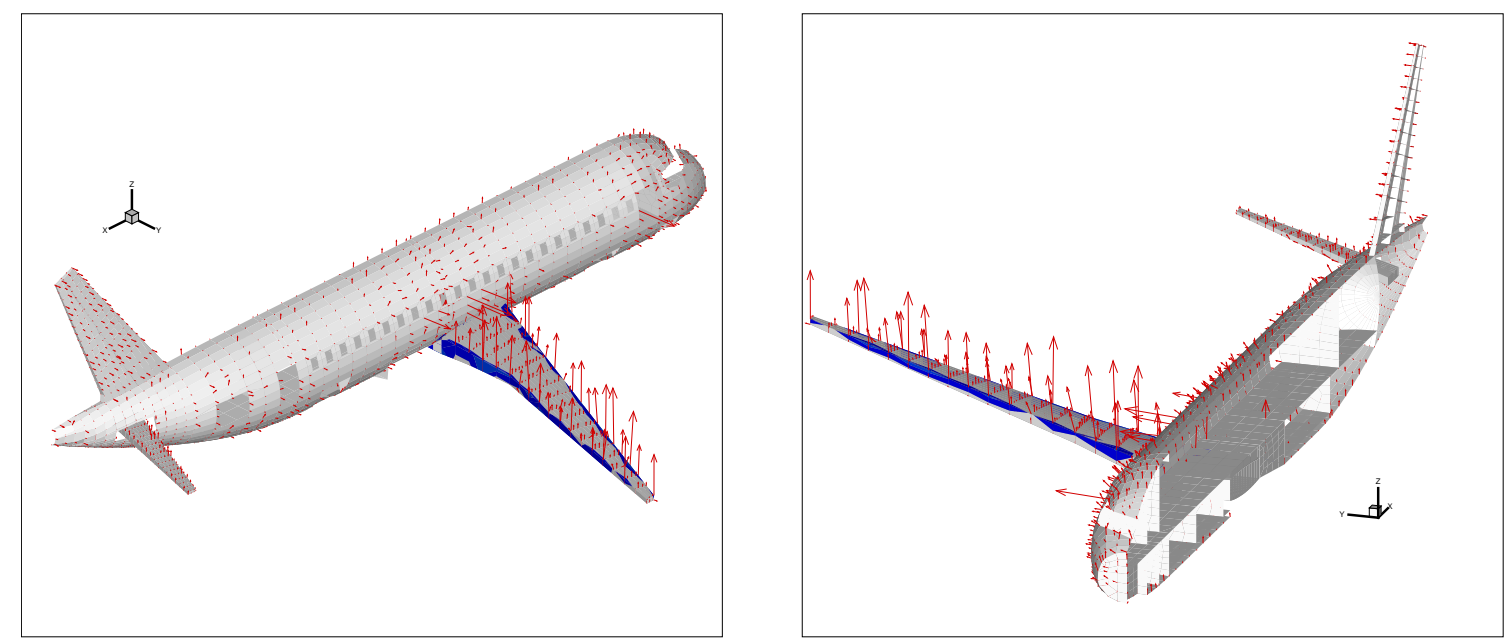

Abbildung 103. Die transformierten Kräfte auf dem Strukturmodell bei Interpolation über die „Partition of Unity“ Methode.

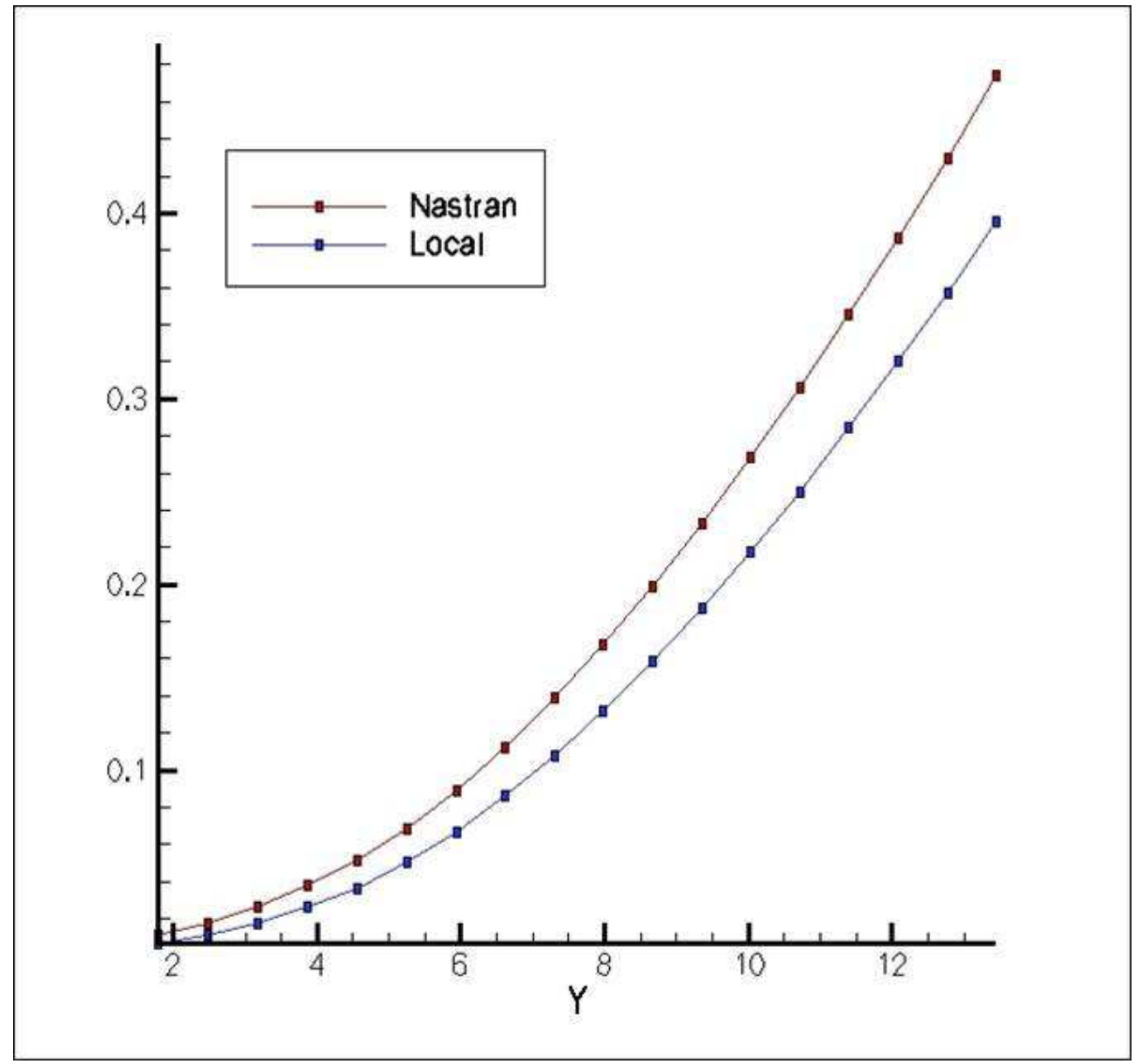

Abbildung 104. Vergleich der vertikalen Verschiebungen entlang der Spannweite des Flügels. Die Kopplung über die „Partition of Unity“ Methode wird hier als lokale Methode bezeichnet. 


\section{Zusammenfassung und Ausblick}

\section{Zusammenfassung}

Die vorliegende, interdisziplinäre Arbeit ist im Bereich zwischen Mathematik und Informatik angesiedelt. Es wurden sowohl mathematische Methoden als auch Algorithmen der Informatik entwickelt, implementiert und untersucht. Ferner bedurfte es einer engen Zusammenarbeit mit den Ingenieurswissenschaften.

Zunächst seien die mehr softwaretechnisch-algorithmischen Arbeiten genannt. MpCCI wurde generell mit Schnittstellen versehen, die mehrere Arten von benutzerdefinierten Interpolationen ermöglichen. Zusätzlich wurden Verfahren implementiert, welche die Gitterdaten in der Art aufbereiten, dass sie in der Interpolationsbibliothek verwendet werden können. Die Transformationsalgorithmen wurden in Verfahren eingebunden, so dass MpCCI bei einem Datenaustausch auf Grundlage der angeschlossenen Interpolationen auf diese zurückgreifen kann. Das heißt, die Werte, die aus den Simulationscodes über $\mathrm{MpCCI}$ an die Interpolationsalgorithmen gegeben werden, werden entsprechend angepasst und die an MpCCI als Input für die Simulationscodes aus den Interpolationsalgorithmen resultierenden Werte werden so umgewandelt, dass MpCCI bzw. die Simulationscodes mit diesen Werten arbeiten können. Hierbei musste besonders die eventuell parallele Situation beachtet werden. Nach Integration der Interpolationen in die Kopplungsbibliothek wurde die Schnittstelle besonders im Hinblick auf die Parallelisierung im Umfeld vorhandener Anwendungen getestet. Datenstrukturen und rechenzeitrelevante Verfahren, die zur Vorbereitung der Daten zur Interpolation und Kopplung dienen, sind modifiziert worden, um generell die Anwendbarkeit auszudehnen.

Die im Folgenden genannten Punkte fassen die neu entwickelten und validierten mathematischen Algorithmen der Arbeit kurz zusammen.

Schnelle Verfahren zur Interpolation mit radialen Basisfunktionen mit minimierten Speicherplatz-Anforderungen sind das Thema des zweiten großen Bereichs neu entwickelter und implementierter Methoden. In diesem Zusammenhang sei besonders der für die „Partition of Unity“ Methode entwickelte Algorithmus zur Partitionierung des Berechnungsgebiets auf Grundlage der Stützstellen genannt.

Der dritte große Teil neuer Verfahren beinhaltet die Entwicklung von Algorithmen, die Zusatzbedingungen, wie sie in der Aeroelastik und auch in anderen Bereichen benötigt werden, realisieren. Diese neuen Ansätze zur Erfüllung gegebener Zusatzbedingungen sind zur Kombination mit den schnellen Algorithmen weiterentwickelt worden und konnten so auch an den großen Beispielproblemen aus der Aeroelastik validiert werden.

Die folgende Aufzählung listet die entscheidenden neuen Algorithmen anhand der dadurch gewonnenen neuen Features auf: 
- Schnittstelle zu der parallelen Kopplungsbibliothek MpCCI

- Parallelisierung der Algorithmen, angelehnt an die parallele Datenstruktur von MpCCI

- Zusatzbedingungen zur Konservativität

- Zusatzbedingungen zur Erhaltung der Arbeit

- Zusatzbedingungen zur Konservativität und zur Erhaltung der Arbeit

- Zusatzbedingungen für Rotationen

- „Partition of Unity“ Methode

- „Partition of Unity“ Methode kombiniert mit Zusatzbedingungen zur Konservativität

- „Partition of Unity“ Methode kombiniert mit Zusatzbedingungen zur Erhaltung der Arbeit

- „Partition of Unity“ Methode kombiniert mit Zusatzbedingungen zur Konservativität und zur Erhaltung der Arbeit

- „Partition of Unity“ Methode kombiniert mit Zusatzbedingungen für Rotationen

\section{Diskussion}

Die implementierte Schnittstelle zu MpCCI hat sich besonders aus den zwei folgenden Gründen als sehr sinnvoll erwiesen. Ein Grund ist, dass Anwendungen, die bereits mit MpCCI gekoppelt sind, die Interpolationen aus der entwickelten Interpolationsbibliothek ohne großen Zusatzaufwand nutzen können. Es müssen keine neuen Schnittstellen implementiert werden, es genügt, die Interpolationsbibliothek zu linken und die Interpolationen im Inputfile anzuwählen. Die Interpolationen können so auch auf einfache Weise mit den bereits in MpCCI implementierten Interpolationen verglichen werden. Ein weiterer Grund ist mit Blick auf parallele Anwendungen zu finden. MpCCI bietet für parallele Anwendungen eine einfach zu verwendende, transparente Schnittstelle, deren Nutzung bei parallelen Datenstrukturen von großem Vorteil ist. Die Daten müssen zwar mit dem in dieser Arbeit entwickelten Transformationstool von der parallelen Struktur in die von der Interpolationsbibliothek benötigte Struktur umgewandelt werden, die Schnittstelle zu der parallelen Anwendung wird aber durch MpCCI realisiert.

Ein Nachteil der Anbindung an MpCCI ist, dass die Datenstrukturen in der Interpolationsbibliothek stark an MpCCI angepasst werden mussten, um zeitaufwendige und fehleranfällige Umstrukturierung zwischen MpCCI und der Interpolationsbibliothek zu vermeiden. In einigen Algorithmen der Interpolationsbibliothek wäre eine freie Wahl der Datenstruktur in Bezug auf den Programmieraufwand und den Speicherplatzbedarf von Vorteil gewesen. Da die Interpolationsbibliothek auch ohne MpCCI genutzt werden kann, ist die Schnittstelle als Zusatz zu sehen und die Zukunft wird zeigen, welche der beiden Nutzungsmöglichkeiten bei den Anwendungen überwiegen wird. 
Durch die „Partition of Unity“ Methode sind Probleme mit über zehntausend Stützstellen überhaupt erst berechenbar, abgesehen von Rechnungen bei denen das lineare Gleichungssystem über BICGStab gelöst wird, was durchaus Rechenzeiten von mehreren Tagen in Anspruch nehmen kann. So eröffnet die „Partition of Unity“ Methode überhaupt erst die Möglichkeit der Anwendung der radialen Basisfunktionen zur Interpolation bei derart großen Problemen.

Ein Nachteil der hier entwickelten Partitionierung ist, dass der Algorithmus in einigen Fällen über einstellbare Parameter an das Problem angepasst werden muss, um vernünftige Lösungen zu erhalten. Als Beispiel hierfür können im dreidimensionalen Raum die Spezialfälle genannt werden, in welchen alle Punkte einer Partition auf einer Ebene oder sogar auf einer Geraden liegen. Dies würde z.B. bei der gewünschten Verwendung von linearen oder quadratischen polynomialen Termen zu einem nicht eindeutig lösbaren Gleichungssystem führen. In einem solchen Fall müssen die Partitionen, sofern möglich, so lange angepasst werden, bis keine Partition nur Punkte auf einer Ebene bzw. sogar Geraden enthält oder die Verwendung von linearen polynomialen Termen muss aufgegeben werden.

Auch die Konfiguration bei Kombination der "Partition of Unity" Methode mit Zusatzbedingungen zur Konservativität und zur Erhaltung der Arbeit ist hinsichtlich der Partitionierung nicht immer ganz einfach, da in jeder Partition Auswertungsstellen-Punkte vorhanden sein müssen, um diese Bedingungen zu gewährleisten. Da bei einer Strömung-Struktur-Kopplung in der Aeroelastik, wie in den hier gezeigten Beispielen, oft eine sehr viel größere Anzahl von Strömungsknoten als von Strukturknoten zu finden ist, wobei die Strukturknoten hier die Auswertungsstellen-Punkte sind, ist eine derartige Aufteilung oft nur über in dieser Arbeit implementierte Zusatzalgorithmen $\mathrm{zu}$ erreichen.

Im Folgenden werden die Ergebnisse der Verfahren anhand der in dieser Arbeit durchgeführten Anwendungen gegenüber gestellt.

In den Einzeltests und der gekoppelten Simulation ergaben die Interpolationen über die „Partition of Unity“ Methode ebenso gute wie, teilweise sogar bessere Resultate als die globale Interpolation mit radialen Basisfunktionen, daher ist die Interpolationen über die „Partition of Unity“ Methode der globalen Interpolation mit radialen Basisfunktionen vorzuziehen wegen ihres bei hohen Anzahlen von Stützstellen erheblichen Vorteils bei Rechenzeit- und Speicherplatzbedarf.

Die Verfahren mit Zusatzbedingungen zur Konservativität und zur Erhaltung der Arbeit ergeben allerdings in der Regel physikalisch weniger sinnvolle Resultate als die Transformation über die Transponierte der Kopplungsmatrix in den Strömung-Struktur-Kopplungen im Bereich der Aeroelastik. Die großen Unterschiede bei der Transformation der Kräfte über die Transponierte der Kopplungsmatrix bei der globalen Interpolation mit verschiedenen radialen Basisfunktionen (siehe Kapitel IV Abschnitt 1), zeigen sich bei Anwendung der „Partition of Unity“ Methode nicht mehr. Mit der „Partition of 
Unity" Methode werden auch bei Verwendung der Wendland 1 und Wendland 2 Funktion mit der Transformation der Kräfte über die Transponierte der Kopplungsmatrix physikalisch sinnvolle Resultate erhalten. Damit ist das Problem bei der Transformation der Kräfte über die Transponierte der Kopplungsmatrix, das am Anfang dieser Arbeit durch die Zusatzbedingungen beseitigt werden sollte durch die Anwendung der „Partition of Unity“ Methode gelöst worden. Die Zusatzbedingungen zur Interpolation der Rotationen haben sich aber als sehr nützlich herausgestellt, besonders bei Kopplungen, wo von dem Strukturmodell über die Deformationen nur wenige Informationen an das Strömungsmodell gegeben werden können.

Die Rotationswerte an den Knoten in der globalen Interpolation über Zusatzbedingungen zu verwenden, ist immer dann sinnvoll, wenn die für die Interpolation zur Verfügung stehenden Verschiebungen als Information für die Interpolation nicht ausreichend sind oder noch ergänzt werden sollen, um die Interpolation zu verfeinern. Dies gilt sowohl für die Interpolationen über die "Partition of Unity“ Methode als auch für die globale Interpolation mit radialen Basisfunktionen. Die Algorithmen sind nur für kleine Winkel anwendbar und bieten sich damit für eine dynamische Kopplung im Zeitbereich an.

\section{Ausblick}

Bei EADS, DLR und SCAI wird die entwickelte Interpolationsbibiliothek sowohl eigenständig als auch in Verbindung mit MpCCI zur räumlichen Kopplung bei Strömung-Struktur Problemen und anderen multidisziplinären Simulationen eingesetzt. So werden weitere Erfahrungen durch Vergleiche der zahlreichen Features gewonnen, die bis zum Abschluss dieser Arbeit wegen der Vielzahl der Möglichkeiten noch nicht umfassend angewendet werden konnten. Aus den Vergleichen, die damit gezogen werden können, wird eine bessere Spezifizierung der besten Konfiguration für eine gegebene Problemklasse möglich sein.

Die „Nearest Neighbour" Suchalgorithmen aus ANN sollten in Zukunft durch eigene Suchalgorithmen auf Grundlage der in dieser Arbeit entwickelten und bei der „Partition of Unity“ Methode entwickelten Überdeckung des Gebietes ersetzt werden. Die Performance von ANN wird sicherlich nicht erreicht, aber es sollte für eine kommerzielle Nutzung des Interpolationstools aus rechtlichen Gründen möglich sein, ANN nicht benutzen zu müssen. Für die Ersetzung spricht auch, dass bei der Nutzung von "Partition of Unity“ nur einmal Suchstrukturen aufgebaut werden.

Ferner ist eine weitere Geschwindigkeitserhöhung durch eine modifizierte Gebietszerlegung möglich und in der Zukunft erstrebenswert.

Ein weiteres Verfahren neben der „Partition of Unity“ Methode zur Reduzierung der Rechenzeit im Rahmen der Interpolation mit radialen Basisfunktion ist ein „Greedy" Verfahren. Bei einem „Greedy" Verfahren wird im ersten 
Schritt ein kleiner Teil der Stützstellen ausgewählt und mit diesen Stützstellen wird eine Interpolation auf die restlichen Stützstellen durchgeführt. Dann werden die Differenzen zwischen den auf den restlichen Stützstellen angeordneten Werten und den über die Interpolation berechneten Werten gebildet. Auf Grundlage dieser Residuen werden weitere Stützstellen für den nächsten Schritt ausgewählt. Im zweiten Schritt wird dann mit der vergrößerten Stützstellen-Untermenge wie im ersten Schritt verfahren. Der Algorithmus wird abgebrochen, sobald die Residuen unter einer vorher definierten Schranke liegen. Neben den Untermengen können auch die radiale Basisfunktion und ihr Trägerradius unterschiedlich gewählt werden.

Für das "Greedy" Verfahren in Verbindung mit radialen Basisfunktionen sind im Rahmen dieser Arbeit bereits Konzepte entwickelt und erste Implementationen getestet worden. Die Algorithmen waren im Bereich der Aeroelastik nicht vielversprechend.

Wie schon in der Einleitung beschrieben, enthält die entwickelte Bibliothek auch Approximationsverfahren, die unter anderem bereits auf verschiedenen Gebieten zu Parameterstudien verwendet wurden. Eine neue Idee ist, diese Algorithmen im Anlagenbau bei der Prozessautomation zur Approximation von Größen einzusetzen, die auf Grundlage der physikalischen Theorie schwer zu berechnen sind.

\section{Danksagung}

An erster Stelle danke ich Priv. Doz. Dr. H. Wendland vom Institut für Numerische und Angewandte Mathematik der Georg-August-Universität Göttingen für die wertvolle Unterstützung durch wegweisende Anregungen und Diskussionen, mit denen er diese Arbeit förderte, seine hervorragende wissenschaftliche Betreuung und die kritische Durchsicht meiner Arbeit.

Herrn Prof. Dr. R. Schaback vom Institut für Numerische und Angewandte Mathematik der Georg-August-Universität Göttingen danke ich für die Übernahme des Korreferats.

Herzlich danke ich Dr. A. Beckert von der European Aeronautic Defence and Space Company (EADS) für sein persönliches Engagement, das die Grundlage zu dieser Arbeit geschaffen hat, seine vielfältigen Anregungen und seine sachkundige Unterstützung. Ich danke ihm auch für die Initiierung und Betreuung der Diplomarbeit von Filippo Mattioni, der die in dieser Arbeit entwickelten Algorithmen im Rahmen seiner Diplomarbeit angewendet und getestet hat und dessen Ergebnisse für meine Arbeit von großer Bedeutung waren.

Diese Arbeit entstand am Institut für Algorithmen und Wissenschaftliches Rechnen (SCAI) der Fraunhofer-Gesellschaft. Sie wurde vom Institut für Aeroelastik des Deutschen Zentrums für Luft- und Raumfahrt gefördert. Herrn Dr. H. Hönlinger, dem Leiter des Instituts für Aeroelastik des Deutschen Zentrums für Luft- und Raumfahrt, danke ich herzlich für die Förderung dieser Arbeit und das stetige Interesse am Fortgang dieser Arbeit. 
Herrn Dr.-Ing. F. Kießling vom Institut für Aeroelastik des Deutschen Zentrums für Luft- und Raumfahrt danke ich sehr für seine konstruktive Unterstützung und seine kritischen Denkanstöße, die einen wesentlichen Beitrag zu dieser Arbeit gegeben haben.

Herrn Dr. P. Post danke ich für seine Hilfe in allen Fragen der Softwaretechnik, für die er sich immer Zeit genommen hat.

Herrn Jens Neumann vom Institut für Aeroelastik des Deutschen Zentrums für Luft- und Raumfahrt danke ich für die produktive und kollegiale Zusammenarbeit.

Die vielen netten und hilfsbereiten Kollegen am Institut für Aeroelastik des Deutschen Zentrums für Luft- und Raumfahrt und am Institut für Algorithmen und Wissenschaftliches Rechnen der Fraunhofer-Gesellschaft haben mich in vielfältiger Weise unterstützt, wofür ich mich an dieser Stelle auch bedanken möchte.

Dem Institut für Algorithmen und Wissenschaftliches Rechnen der Fraunhofer-Gesellschaft danke ich für die Überlassung der verschiedenen Anwendungsdaten außerhalb des aeroelastischen Bereichs. Herrn R. Sonnenschein danke ich für die Testfälle aus dem Bereich Sprühlackierung. 


\section{Appendix}

\section{A Tabellen zu den Abbildungen der Anwendungen}

Im folgenden werden die in den Tabellen 20 bis 22 aufgeführten Angaben kurz beschrieben.

In der ersten Spalte der Tabellen ist die Abbildungsnummer zu der Rechnung angegeben. Die zweite Spalte der Tabellen kennzeichnet, welche Knoten der Anwendung als Stützstellen für die jeweilige Interpolation dienen, ob zum Beispiel bei einer Strömung-Struktur-Kopplung die Struktur- oder Strömungsknoten als Stützstellen verwendet werden.

Die Abkürzung für den verwendeten Algorithmus und die evtl. Partitionseinteilungen bei der "Partition of Unity“ Methode werden in den nächsten Spalten angegeben. In der zusätzlichen Tabelle 19 in diesem Anhang sind die zu den Abbildungen angegebenen Abkürzungen für die Algorithmen erläutert. Die Partitionseinteilungen werden in den Tabellen so spezifiziert, dass die Spalte mit dem Buchstaben $p$ in der Überschrift die Zahl zeigt, die die Anzahl der Stützstellen darstellen würde, wenn kein Überlapp konstruiert würde. Die Spalte mit der Überschrift $K R$ gibt die Anzahl der Koordinatenrichtungen an, die für die Sortierungen zur Bildung der Buckets verwendet werden. In der Spalte mit dem Buchstaben $o$ in der Überschrift wird der Überlapp spezifiziert. Die Beschreibung der für die Partitionierung relevanten Größen ist im Kapitel II Abschnitt 3.2 genau erklärt.

Die Spalte mit der Überschrift $R B F$ enthält die Abkürzung für die radiale Basisfunktion und die darauffolgende Spalte die Anzahl der polynomialen Terme. Die unter $N N$ angegebene Nummer der nächsten Nachbarn ist die Anzahl der Stützstellen, die mindestens für jede Stütz- und Auswertungsstelle im kompakten Träger liegen müssen. Der Radius wird so bestimmt, dass die Stützstellen innerhalb des Radius liegen, und nicht ein Teil der Stützstellen auf dem Radius liegt. Das heißt, es gibt immer eine offene Umgebung für jeden Punkt auf dem Radius, so dass keine Stützstelle dieser mindestens im Radius liegenden Stützstellen in dieser Umgebung liegt. Dieser Radius wird mit dem in der nächsten Spalte spezifizierten Faktor multipliziert. Das Ergebnis wird in der letzten Spalte angegeben. Wenn z.B. die unter $N N$ angegebene Nummer 10 ist und der Faktor auf 5.0 steht, so ist bei der beschriebenen Anwendung der Trägerradius für die Funktionen mit kompaktem Träger das fünffache des Radius, in dem für jede Stütz- und Auswertungsstelle mindestens zehn weitere Stützstellen liegen. 


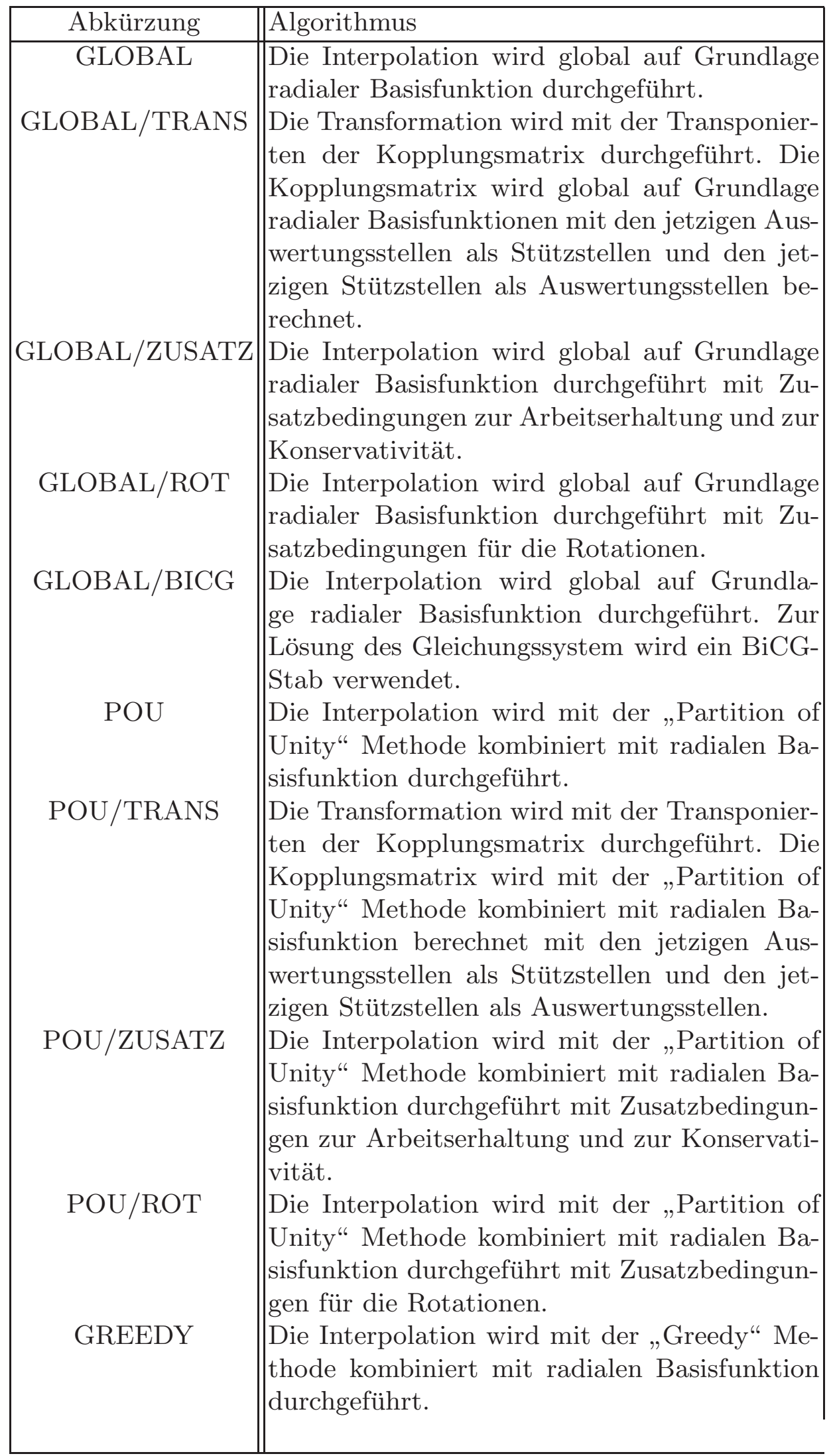

Tabelle 19. Die Aufstellung der in den Anwendungsbeispielen getesteten Algorithmen und ihrer in den folgenden Tabellen verwendeten Abkürzungen. 


\begin{tabular}{|c|c|c|c|c|c|c|c|c|c|c|}
\hline Abb.-Nr. & Stütz. & Algorithmus & $p$ & $\mathrm{KR}$ & $O$ & RBF & pT & $\mathrm{NN}$ & Faktor & Radius \\
\hline $\begin{array}{c}22 \\
\text { Abb. links }\end{array}$ & Strukt. & GLOBAL & - & - & - & TPS & 1 & - & - & - \\
\hline $\begin{array}{c}22 \\
\text { Abb. rechts }\end{array}$ & Strukt. & \begin{tabular}{|c|} 
GLOBAL/ \\
TRANS
\end{tabular} & - & - & - & TPS & 1 & - & - & - \\
\hline $\begin{array}{c}23 \\
\text { Abb. links }\end{array}$ & Strukt. & GLOBAL & - & - & - & TPS4 & 1 & - & - & - \\
\hline $\begin{array}{c}23 \\
\text { Abb. rechts }\end{array}$ & Strukt. & $\begin{array}{c}\text { GLOBAL/ } \\
\text { TRANS }\end{array}$ & - & - & - & TPS4 & 1 & - & - & - \\
\hline $\begin{array}{c}24, \\
\text { Abb. links }\end{array}$ & |Strukt. & GLOBAL & - & - & - & VS & 1 & - & - & - \\
\hline $\begin{array}{c}24, \\
\text { Abb. rechts }\end{array}$ & Strukt. & $\begin{array}{c}\text { GLOBAL/ } \\
\text { TRANS }\end{array}$ & - & - & - & VS & 1 & - & - & - \\
\hline $\begin{array}{c}25 \\
\text { Abb. links }\end{array}$ & IStrukt. & GLC & - & - & - & VS3 & 1 & - & - & - \\
\hline $\begin{array}{c}25 \\
\text { Abb. rechts }\end{array}$ & Strukt. & $\begin{array}{c}\text { GLOBAL/ } \\
\text { TRANS }\end{array}$ & - & - & - & VS3 & 1 & - & - & - \\
\hline $\begin{array}{c}26, \\
\text { Abb. links }\end{array}$ & |Strukt. & GLOBAL & - & - & - & $\mathrm{EH}$ & 1 & 10 & 10.0 & $2.43 e+0 \mathrm{c}$ \\
\hline $\begin{array}{c}26, \\
\text { Abb. rechts }\end{array}$ & Strukt. & $\begin{array}{c}\text { GLOBAL/ } \\
\text { TRANS }\end{array}$ & - & - & - & $\mathrm{EH}$ & 1 & 10 & 10.0 & $2.43 e+0 \mathrm{c}$ \\
\hline $\begin{array}{c}27, \\
\text { Abb. links }\end{array}$ & Strukt. & GLOBAL & - & - & - & W1 & 1 & 10 & 10.0 & $1.21 e+00$ \\
\hline $\begin{array}{c}27 \\
\text { Abb. rechts }\end{array}$ & Strukt. & $\begin{array}{c}\text { GLOBAL/ } \\
\text { TRANS }\end{array}$ & - & - & - & W1 & 1 & 10 & 10.0 & $1.21 e+00$ \\
\hline $\begin{array}{c}28 \\
\text { Abb. links }\end{array}$ & Strukt. & GLOBAL & - & - & - & W2 & 1 & 10 & 10.0 & $1.21 e+00$ \\
\hline $\begin{array}{c}28 \\
\text { Abb. rechts }\end{array}$ & Strukt. & \begin{tabular}{|c|} 
GLOBAL/ \\
TRANS
\end{tabular} & - & - & - & W2 & 1 & 10 & 10.0 & $1.21 e+00$ \\
\hline $\begin{array}{c}29, \\
\text { Abb. links }\end{array}$ & Strukt. & GLOBAL & - & - & - & W3 & 1 & 10 & 10.0 & $1.21 e+00$ \\
\hline $\begin{array}{c}29 \\
\text { Abb. rechts }\end{array}$ & Strukt. & $\begin{array}{c}\text { GLOBAL/ } \\
\text { TRANS }\end{array}$ & - & - & 1 & W3 & 1 & 10 & 10.0 & $1.21 e+00$ \\
\hline
\end{tabular}

Tabelle 20. Die Aufstellung der Abbildungen mit den Spezifikationen des jeweiligen Verfahrens. 


\begin{tabular}{|c|c|c|c|c|c|c|c|c|c|c|}
\hline Abb.-Nr. & Stütz. & Algorithmus & $p$ & $\mathrm{KR}$ & $O$ & RBF & pT & $\mathrm{NN}$ & Faktor & Radius \\
\hline $\begin{array}{c}30, \\
\text { Abb. links }\end{array}$ & Ström. & $\begin{array}{l}\text { GLOBAL/ } \\
\text { ZUSATZ }\end{array}$ & - & - & - & TPS & 1 & - & - & - \\
\hline $\begin{array}{c}30, \\
\text { Abb. rechts }\end{array}$ & |Ström. & $\begin{array}{l}\text { GLOBAL/ } \\
\text { ZUSATZ }\end{array}$ & - & - & -1 & TPS4 & 1 & - & - & - \\
\hline $\begin{array}{c}31 \\
\text { Abb. links }\end{array}$ & ||Ström. & $\begin{array}{l}\text { GLOBAL/ } \\
\text { ZUSATZ }\end{array}$ & - & - & - & VS & 1 & - & - & - \\
\hline $\begin{array}{c}31 \\
\text { Abb. rechts }\end{array}$ & |Ström. & $\begin{array}{l}\text { GLOBAL/ } \\
\text { ZUSATZ }\end{array}$ & - & - & - & VS3 & 1 & - & - & - \\
\hline $\begin{array}{c}32, \\
\text { Abb. links }\end{array}$ & Ström. & $\begin{array}{c}\text { GLOBAL/ } \\
\text { ZUSATZ }\end{array}$ & - & - & - & $\mathrm{EH}$ & 1 & 10 & 10.0 & $2.43 e+00$ \\
\hline $\begin{array}{c}32, \\
\text { Abb. rechts }\end{array}$ & ||Ström. & $\begin{array}{l}\text { GLOBAL/ } \\
\text { ZUSATZ }\end{array}$ & - & - & - & W1 & 1 & 10 & 10.0 & $1.21 e+00$ \\
\hline $\begin{array}{c}33 \\
\text { Abb. links }\end{array}$ & |Ström. & $\begin{array}{l}\text { GLOBAL/ } \\
\text { ZUSATZ }\end{array}$ & - & - & - & W2 & 1 & 10 & 10.0 & $1.21 e+00$ \\
\hline $\begin{array}{c}33 \\
\text { Abb. rechts }\end{array}$ & ||Ström. & $\begin{array}{l}\text { GLOBAL/ } \\
\text { ZUSATZ }\end{array}$ & - & - & - & W3 & 1 & 10 & 10.0 & $1.21 e+00$ \\
\hline 35 & Strukt. & $\begin{array}{c}\text { GLOBAL/ } \\
\text { ROT }\end{array}$ & - & - & - & W2 & 0 & 10 & 10.0 & $7.37 e+00$ \\
\hline 36 & |Strukt. & $\begin{array}{c}\text { GLOBAL/ } \\
\text { ROT }\end{array}$ & - & - & - & W2 & 0 & 10 & 10.0 & $7.37 e+00$ \\
\hline 37 & |Strukt. & GLOBAL & - & - & - & W2 & 0 & 10 & 10.0 & $7.37 e+00$ \\
\hline 38 & Strukt. & GLOBAL & - & - & - & W2 & 0 & 10 & 10.0 & $7.37 e+00$ \\
\hline $\begin{array}{c}44, \\
\text { Abb. links, oben }\end{array}$ & ||Strukt. & $\begin{array}{l}\text { GLOBAL/ } \\
\text { BICG }\end{array}$ & - & - & - & $\mathrm{EH}$ & 4 & 5 & 1.0 & $3.13 e+00$ \\
\hline $\begin{array}{c}44, \\
\text { Abb. links, unten }\end{array}$ & Strukt. & $\begin{array}{c}\text { GLOBAL/ } \\
\text { BICG }\end{array}$ & - & - & - & $\mathrm{EH}$ & 4 & 40 & 1.0 & $4.21 e+00$ \\
\hline $\begin{array}{c}44, \\
\text { Abb. rechts, oben }\end{array}$ & Strukt. & $\begin{array}{c}\text { GLOBAL/ } \\
\text { BICG }\end{array}$ & - & - & - & $\mathrm{EH}$ & 0 & 20 & 1.0 & $3.19 e+00$ \\
\hline $\begin{array}{c}44, \\
\text { Abb. rechts, unten }\end{array}$ & Strukt. & $\begin{array}{c}\text { GLOBAL/ } \\
\text { BICG }\end{array}$ & - & - & - & $\mathrm{EH}$ & 10 & 20 & 1.0 & $3.19 e+00$ \\
\hline $\begin{array}{c}45, \\
\text { Abb. rechts, oben }\end{array}$ & Strukt. & $\begin{array}{c}\text { GLOBAL/ } \\
\text { BICG }\end{array}$ & - & - & - & VS & 10 & - & - & - \\
\hline $\begin{array}{c}45, \\
\text { Abb. rechts, unten }\end{array}$ & Strukt. & $\mathrm{POU}$ & 8 & 3 & 3 & VS & 10 & - & - & - \\
\hline $\begin{array}{c}45, \\
\text { Abb. links, oben }\end{array}$ & Strukt. & $\begin{array}{c}\text { GLOBAL/ } \\
\text { BICG }\end{array}$ & - & - & - & VS & 10 & - & - & - \\
\hline $\begin{array}{c}45, \\
\text { Abb. links, unten }\end{array}$ & Strukt. & $\mathrm{POU}$ & 8 & 3 & 3 & VS & 10 & - & - & - \\
\hline $\begin{array}{c}45, \\
\text { Abb. rechts, oben }\end{array}$ & |Strukt. & $\begin{array}{l}\text { GLOBAL/ } \\
\text { BICG }\end{array}$ & - & - & - & VS & 10 & - & - & - \\
\hline $\begin{array}{c}45, \\
\text { Abb. rechts, unten }\end{array}$ & Strukt. & $\mathrm{POU}$ & 8 & 3 & 3 & VS & 10 & - & - & - \\
\hline 46 & Strukt. & $\overline{\mathrm{POU}}$ & 10 & 3 & 3 & W3 & 1 & 291 & 10.0 & $7.98 e+01$ \\
\hline $\begin{array}{c}47, \\
\text { Abb. links }\end{array}$ & |Strukt. & $\mathrm{POU}$ & 10 & 3 & 3 & W3 & 1 & 291 & 10.0 & $7.98 e+01$ \\
\hline
\end{tabular}

Tabelle 21. Die Aufstellung der Abbildungen mit den Spezifikationen des jeweiligen Verfahrens. 


\begin{tabular}{|c|c|c|c|c|c|c|c|c|c|c|}
\hline Abb.-Nr. & Stütz. & Algorithmus & $p$ & KR & $o$ & $\overline{\mathrm{RBF}}$ & pT & $\mathrm{NN}$ & Faktor & Radius \\
\hline 49 & \begin{tabular}{|c|} 
Knoten \\
äußeres Rohr
\end{tabular} & POU & 8 & 3 & 3 & VS & 4 & - & - & - \\
\hline 50 & \begin{tabular}{|c|} 
Knoten \\
äußeres Rohr
\end{tabular} & $\mathrm{POU}$ & 8 & 3 & 3 & VS & 4 & - & - & - \\
\hline 51 & \begin{tabular}{|c|} 
Knoten \\
äußeres Rohr
\end{tabular} & $\mathrm{POU}$ & 8 & 3 & 3 & VS & 4 & - & - & - \\
\hline 52 & \begin{tabular}{|c|} 
Knoten \\
äußeres Rohr
\end{tabular} & $\mathrm{POU}$ & 8 & 3 & 3 & VS & 4 & - & - & - \\
\hline 53 & $\begin{array}{c}\text { Knoten } \\
\text { äußeres Rohr }\end{array}$ & $\mathrm{POU}$ & 8 & 3 & 3 & VS & 4 & - & - & - \\
\hline 54 & \begin{tabular}{|c} 
Knoten \\
äußeres Rohr
\end{tabular} & POU & 8 & 3 & 3 & VS & 4 & - & - & - \\
\hline 58 & Ström. & $\mathrm{POU}$ & 2 & 2 & 2 & W2 & 0 & 32 & 1.0 & $1.89 e-0$ \\
\hline 57 & Ström. & $\mathrm{POU}$ & 2 & 2 & 2 & W2 & 0 & 32 & 1.0 & $1.89 e-01$ \\
\hline 59 & Ström. & POU & 2 & 2 & 2 & W1 & 0 & 32 & 1.0 & $1.89 e-0$ \\
\hline 61 & \begin{tabular}{|c|}
$\begin{array}{c}\text { Knoten } \\
\text { großes Blech }\end{array}$ \\
\end{tabular} & $\mathrm{POU}$ & 2 & 3 & 3 & W3 & 4 & 54 & 10.0 & $1.45 e+0$ \\
\hline 63 & Strukt. & $\begin{array}{c}\text { POU/ } \\
\text { TRANS }\end{array}$ & 2 & 3 & 3 & VS & 4 & - & - & - \\
\hline 64 & Strukt. & $\begin{array}{c}\text { POU/ } \\
\text { TRANS }\end{array}$ & 2 & 3 & 3 & VS & 4 & - & - & - \\
\hline 65 & Strukt. & $\begin{array}{c}\text { POU/ } \\
\text { TRANS }\end{array}$ & 2 & 3 & 3 & VS & 4 & - & - & - \\
\hline 66 & Ström. & $\begin{array}{c}\text { POU/ } \\
\text { ZUSATZ }\end{array}$ & 2 & 3 & 3 & VS & 4 & - & - & - \\
\hline 67 & Ström. & $\begin{array}{c}\text { POU/ } \\
\text { ZUSATZ }\end{array}$ & 2 & 3 & 3 & VS & 4 & - & - & - \\
\hline 68 & Ström. & $\begin{array}{c}\text { POU/ } \\
\text { ZUSATZ }\end{array}$ & 2 & 3 & 3 & VS & 4 & - & - & - \\
\hline 71 & Strukt. & $\begin{array}{l}\mathrm{POU} / \\
\mathrm{ROT}\end{array}$ & 2 & 3 & 3 & W2 & 0 & 10 & 10.0 & $1.00 e+0$ \\
\hline 72 & Strukt. & $\begin{array}{l}\mathrm{POU} / \\
\mathrm{ROT}\end{array}$ & 2 & 3 & 3 & W2 & 0 & 10 & 10.0 & $1.00 e+02$ \\
\hline 73 & Strukt. & $\mathrm{POU}$ & 2 & 3 & 3 & W2 & 0 & 10 & 10.0 & $1.00 e+02$ \\
\hline 74 & Strukt. & $\mathrm{POU}$ & 2 & 3 & 3 & W2 & 0 & 10 & 10.0 & $1.00 e+02$ \\
\hline
\end{tabular}

Tabelle 22. Die Aufstellung der Abbildungen mit den Spezifikationen des jeweiligen Verfahrens. 


\section{Literatur}

1. H.-P. Kersken et. al.: AMANDA - A Distributed System for Aircraft Design. Proceedings of EuroPar 2000, Munich.

2. A. Beckert, H. Wendland: Multivariate interpolation in fluid-structureinteraction using radial basis functions, Aerospace Science and Technology (AST), AST5125 - 2/2001, (2001).

3. A. Beckert: Coupling fluid (CFD) and structural (FE) models using finite interpolation elements, Aerospace Science and Technology (AST), AST5082 $1 / 2000,(2000)$.

4. H. Wendland: Scattered Data Approximation. Cambridge Monographs on Applied and Computational Mathematics, Cambridge University Press, Cambridge, January 2005.

5. http://www.mpcci.org

6. H. Wendland: Konstruktion und Untersuchung radialer Basisfunktionen mit kompaktem Träger. Dissertation. Universität Göttingen, 1996.

7. A. Beckert: Ein Beitrag zur Strömung-Struktur-Kopplung für die Berechnung des aeroelastischen Gleichgewichtszustandes. Dissertation TU Braunschweig, 1997.

8. R. Ahrem, M.G. Hackenberg, P. Post, R. Redler, J. Roggenbuck: Specification of MpCCI Version 1.0 GMD-SCAI, Sankt Augustin, 2000

9. D. M. Mount: ANN Programming Manual, Department of Computer Science and Institute for Advanced Computer Studies, University of Maryland, College Park, Maryland, 1998.

10. H. Wendland: Fast evaluation of radial basis functions: Methods based on partition of unity, in: C. K. Chui, L. L. Schumaker, and J. Stöckler (eds), Approximation Theory X: Wavelets, Splines, and Applications, Vanderbilt University Press, Nashville, 2002, 473-483.

11. H.A. van der Vorst: Bi-CGSTAB: A fast and smoothly converging variant of Bi-CG for the solution of nonsymmetric linear systems, SIAM J. Sci. Stat. Comput., Vol 13(2), pp. 631-644, 1992.

12. K. Stüben: A Review of Algebraic Multigrid. Journal of Computational and Applied Mathematics, 128:281-309, 2001.

13. http://my.unidata.ucar.edu/content/software/netcdf/docs/index.html

14. J. Dongarra and J. Wasniewski: High Performance Linear Algebra Package, LAPACK90 In Panos Pardalos and Sanguthevar Rajasekaran (Eds) Parallel and Distributed Processing 10 IPPS/SPPD'98 Workshops Orlando, Florida, USA, March/April 1998, Proceedings Kluwer academic press, Separate chapter, 30 pages

15. R. Schaback: Reconstruction of Multivariate Functions from scattered data. Vorlesungsskript. Göttingen 1997

16. J. Duchon: Splines minimizing rotation-invariate semi-norms in Sobolev spaces. In W.Schempp and K.Zeller, editors, Constructive Theory of Functions of Several Variables, pages 85-100. Springer, Berlin-Heidelberg, 1979

17. R.L. Hardy: Multiquadric equations of topography and other irregular surfaces. J. Geophys. Res., 76:1905-1915, 1971 
18. C.A. Micchelli: Interpolation of scattered data: distance matrices and conditionally positive definite functions. Constructive Approximation, 2:11-22, 1986

19. G. Tzong, H.H. Chen, K.C. Chang, T. Wu and T.Cebeci: A General Method for Calculating Aero-Structure Interaction on Aircraft Configurations

20. Narcowich, F. J. and J. D. Ward, Generalized Hermite interpolation via matrixvalued conditionally positive definite functions, Mathematics of Computation 63, pp. 661-687, 1994.

21. gcc.gnu.org

22. R. Ahrem, P. Post, K. Wolf: A Communication Library to Couple Simulation Codes on Distributed Systems for Multi-Physics Computations, In ParCo99 Conference Proceedings. Imperial College Press, 1999. Parallel Computing 99 Conference, 17-20 August, Delft, Netherlands.

23. G.A. Kohring and G. Lonsdale, COCOVIS Design, deliverable 3.5, CISPAR ESPRIT Project 20161

24. E.C. Yates: AGARD Standard Aeroelastic Configurations for Dynamic Response, I - Wing 445.6, AGARD Report No. 765, 1988.

25. F. Mattioni: Studies on the effects of global and local spatial coupling methods in static aeroelastic analysis Diploma Thesis, Universita Romà „La Sapienza“

26. Beckert, A., Gerhold, T., Schwamborn, D., Weinman, K.: Applications of the DLR-TAU Code to Fluid-Structure Interaction on Hybrid Grids. ECCOMAS 2000, Barcelona, Spain, 11. - 14. September 2000, Spanish Association for Numerical Methods in Engineering, (2000) 